\title{
A New Test Method for Measuring
}

The Longitudinal and Shear Moduli

of

Fiber-Reinforced Composites

by

Seyed Javad Jalali Mosallam

A Thesis Submitted to the

Faculty of Engineering

in Partial Fulfilment of the Requirments for the Degree of

DOCTOR OF PHILOSOPHY

Major Subject: Civil Engineering

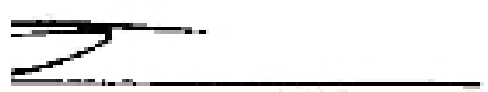

Concordia University

DALHOUSIE UNIVERSITY, DALTECH

Halifax, Nova Scotia 
National Library

of Canada

Acquisitions and Bibliographic Services

395 Wellington Street Ottawa ON K1A ONA Canada
Bibliotheque nationale du Canada

Acquisitions el services bibliographiques

395, ne Wellington

Ottawa ON KIA ON4

Canada
The author has granted a nonexclusive licence allowing the National Library of Canada to reproduce, loan, distribute or sell copies of this thesis in microform, paper or electronic formats.
L'auteur a accordé une licence non exclusive permettant à la Bibliothèque nationale du Canada de reproduire, prêter, distribuer ou vendre des copies de cette thèse sous la forme de microfiche/film, de reproduction sur papier ou sur format électronique.

L'auteur conserve la propriété du droit d'auteur qui protège cette thèse. Ni la thèse ni des extraits substantiels de celle-ci ne doivent être imprimés ou autrement reproduits sans son autorisation. 
In memory of my father

this thesis is dedicated

to

my wife, my mother

and

my son, Pedrum 


\section{TABLE OF CONTENTS}

LIST OF TABLES ................................................................ viii

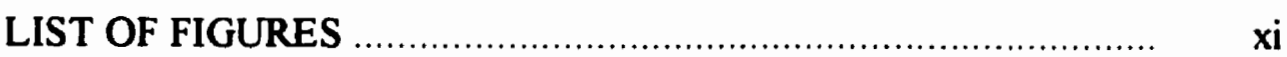

LIST OF SYMBOLS AND ABBREVIATIONS …...................... x xviii

ACKNOWLEDGEMENTS …............................................... xxii

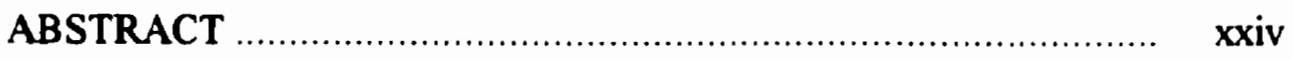

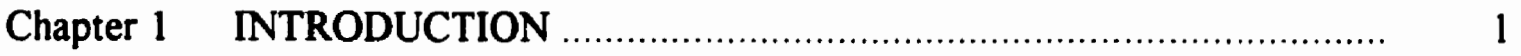

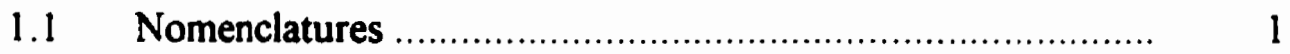

$1.2 \quad$ Evaluation of the Elastic Constants ..................................... 7

1.3 Applicable Theories for the VSM ...................................... 8

1.4 Organization of the Thesis ................................................ 11

Chapter 2 AVAILABLE TEST METHODS REVIEW …............................ 12

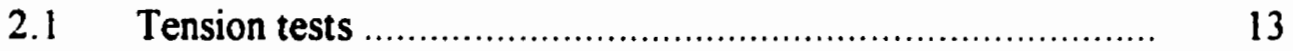

2.1.1 Strip-specimen tension test (ASTM D3039-93) ....... 13

2.1.2 Dogbone-specimen tension test (ASTM D638M-93) . 15

2.1.3 Hydrostatic tension test .......................................... 15

2.1.4 Split disk method (ASTM D2290-92) .................. 16

2.1.5 Filament-wound pressure vessel tension test (ASTM

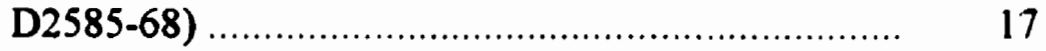

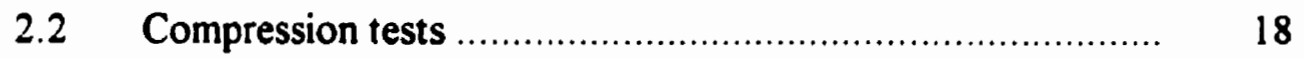

2.2.1 End-loaded specimen (ASTM 695-91) .................... 19

2.2.2 Side-loaded specimen (ASTM D3410-95) ............... 19

2.2.3 Sandwich edgewise compression test (ASTM C364-

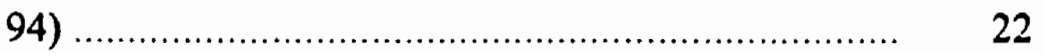

2.2.4 Sandwich beam compression test (ASTM D5467-93) 23

2.2.5 Hydrostatic compression test ( ASTM D2586-68) ... 24

$2.3 \quad$ Flexural test (ASTM D790-93) …................................... 25 
page

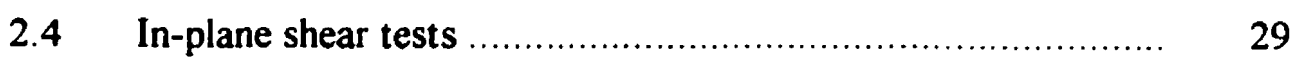

2.4.1 Torsion test on a tube specimen .......................... 30

2.4.2 Rail shear tests (ASTM D4255-83) ...................... 31

2.4.3 $10^{\circ}$ off-axis tensile test .................................... 33

2.4.4 Tensile test on $\pm 45^{\circ}$ symmetric laminate (ASTM D3518-94) ................................................ 35

2.4.5 Iosipescu shear test (ASTM D5379-93) ................ 38

2.5 Through-the-thickness shear tests ................................ 41

2.5.1 Short-beam shear test (ASTM D2344-84) ............ 41

2.5.2 Notched-specimen test method (ASTM D3846-94) .. 42

2.6 Recent achievements .............................................. 43

2.7 Summary and conclusions ...................................... 45

Chapter 3 THE EXACT SOLUTION FOR SIMPLY SUPPORTED BEAMS UNDER THREE-POINT BENDING ................................. 48

3.1 Solution for harmonic loading .................................. 50

3.2 Solution for beam under three-point loading .................... 53

3.3 Numerical investigations ........................................ 57

3.4 Summary and conclusions ...................................... 62

Chapter 4 APPLICATION OF THE THROUGH-THE-THICKNESS

INEXTENSIBILITY THEORY TO ORTHOTROPIC BEAMS ...... 64

4.1 Formulation for a beam subjected to harmonic loading ........ 66

$4.2 \quad$ Numerical investigations ...................................... 70

4.2.1 Half-sine load ............................................ 70

4.2.2 Concentrated load at mid-span .......................... 73

4.3 Summary and conclusions ..................................... 78

Chapter 5 DEVELOPMENT OF THE VARYING-SPAN METHOD ............ 80

5.1 The application of the through-the-thickness inextensibility 


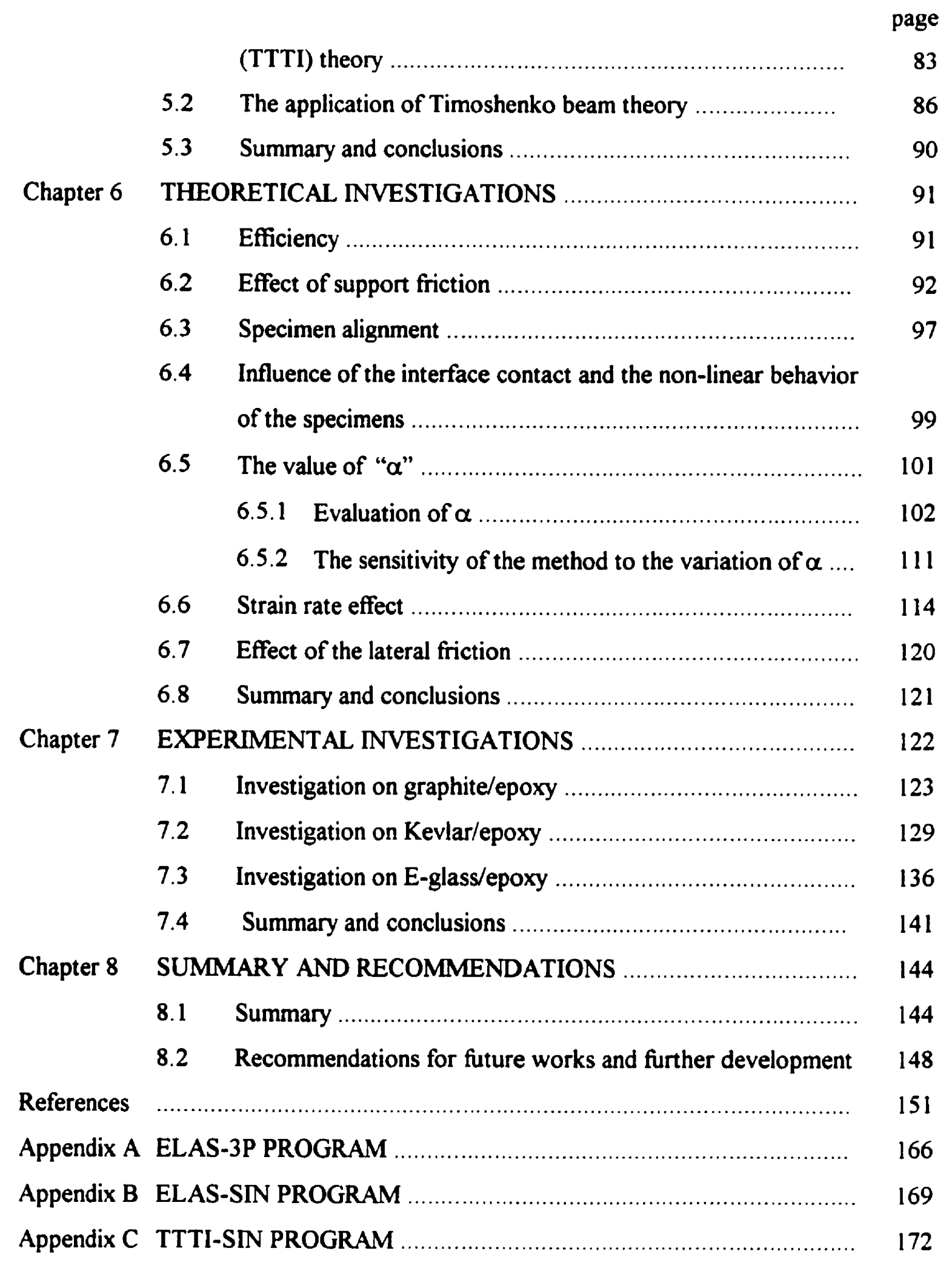


Appendix D ELAS-CON PROGRAM …................................................. 174

Appendix E TTTI-CON PROGRAM ……............................................. 178

Appendix F THE MATERIAL PROPERTIES USED FOR THE

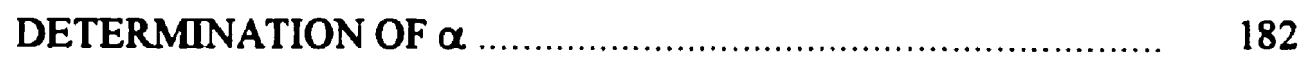

Appendix G VARIATION OF $\alpha$ AND THE ASSOCIATED ERRORS FOR THE MATERIALS OF APPENDIX F ............................................ 186

Appendix H THE VSM APPARATUS ….................................................. 210

Appendix I SPECIFICATIONS OF THE TESTS SPECIMENS ….................... 216

Appendix J LOAD-DEFLECTION CURVES OBTAINED FROM THE VSM .. 225 


\section{LIST OF TABLES}

Page

Table 6.1 The properties of the selected materials from Tsai (1988) ............... 113

Table 6.2 The variation of $\alpha$ and the associated percentage of error ............... $\quad 113$

Table 6.3 Percent of error associated with different stain rate arrangements and the assumed sensitivity to strain rate for long specimens ................. 119

Table 6.4 Percent of error associated with different stain rate arrangements and the assumed sensitivity to strain rate for short specimens ............. 119

Table 6.5 The ratio of the net mid-span deflection obtained from models with and without lateral friction at the supports ................................... 120

Table 7.1 Specifications of strain rate cases for different sets of graphite/epoxy specimens

Table 7.2 Values of $G_{13}$ and $E_{11}$ for different sets of the graphite/epoxy specimens obtained by the VSM (GPa)

Table 7.3 Summary of the results obtained from different test methods and $95 \%$ confidence intervals for graphite/epoxy (GPa)

Table 7.4 Specifications of strain rate cases for different sets of Kevlar/epoxy specimens

Table 7.5 Values of $G_{13}$ and $E_{11}$ for different sets of the Kevlar/Epoxy specimens obtained by the VSM (GPa)

Table 7.6 Summary of the results obtained from different test methods and 95\% confidence intervals for Kevlar/epoxy (GPa)

Table 7.7 Specifications of strain rate cases for different sets of E-glass/epoxy specimens

Table 7.8 Values of $G_{13}$ and $E_{11}$ for different sets of the E-glass/epoxy specimens obtained by the VSM (GPa)

Table 7.9 Summary of the results obtained from different test methods and $95 \%$ confidence intervals for E-glass/epoxy (Gpa) 
Table F.1 The material properties used for the determination of $\alpha$

Table G.1 Results of calculations for short specimens with $\mathrm{b} / \mathrm{h}=1$

Table G.2 Results of calculations for long specimens with $b / h=1$

Table G.3 Results of calculations for short specimens with $b / h=3$

Table G.4 Results of calculations for long specimens with $b / h=3$

198

Table G.5 Results of calculations for short specimens with $\mathrm{b} / \mathrm{h}=5$

202

Table G.6 Results of calculations for long specimens with $\mathrm{b} / \mathrm{h}=5$.

Table I.1 Specifications of graphite/epoxy specimens in GR-1 set for the VSM.

Table I.2 Specifications of graphite/epoxy specimens in GR-2 set for the VSM.

Table I.3 Specifications of graphite/epoxy specimens in GR-3 set for the VSM.

Table I.4 Specifications of graphite/epoxy specimens in GR-4 set for the VSM.

Table I.5 Specifications of Kevlar/epoxy specimens in K-1 set for the VSM ....

Table I.6 Specifications of Kevlar/epoxy specimens in K-2 set for the VSM ...

Table I.7 Specifications of Kevlar/epoxy specimens in K-3 set for the VSM ....

Table I.8 Specifications of Kevlar/epoxy specimens in K-4 set for the VSM ....

Table I.9 Specifications of Kevlar/epoxy specimens in K-5 set for the VSM ....

Table I. 10 Specifications of Kevlar/epoxy specimens in K-6 set for the VSM ....

Table I. 11 Specifications of E-glass/epoxy specimens in GL-1 set for the VSM

Table I.12 Specifications of E-glass /epoxy specimens in GL-2 set for the VSM

Table I.13 Specifications of E-glass/epoxy specimens in GL-3 set for the VSM

Table I.14 Specifications of E-glass/epoxy specimens in GL-4 set for the VSM

Table I.15 Specifications of graphite/epoxy specimens in tensile test

Table I.16 Specifications of E-glass/epoxy specimens in tensile test .....

Table I.17 Specifications of graphite/epoxy specimens in Iosipescu shear test ...

Table I.18 Specifications of Kevlar/epoxy specimens in Iosipescu shear test .....

Table I.19 Specifications of E-glass/epoxy specimens in Iosipescu shear test ....

Table I.20 Specifications of graphite/epoxy specimens in $\pm 45^{\circ}$ shear test 
Table I.21 Specifications of Kevlar/epoxy specimens in $10^{\circ}$ off-axis shear test ... 224 


\section{LIST OF FIGURES}

Figure 1.1 Definition of material principal axes and loading axes for a fiberreinforced lamina ............................................................. $\quad 2$

Figure 1.2 Stress and strain nomenclature …............................................ 3

Figure 1.3 The Coordinates system in beam type problem ............................ 7

$\begin{array}{lll}\text { Figure 2.1 Definition of various elastic moduli and the strength of materials ... } & 12\end{array}$

Figure 2.2 ASTM D3039-93 tensile test .............................................. 14

Figure 2.3 A typical dogbone specimen for tension test ............................... 15

Figure 2.4 Test setup for split disk method ……....................................... 16

Figure 2.5 Filament-wound pressure vessel ........................................... 18

Figure 2.6 ASTM 695-91 fixture for FRPC compression test ....................... 19

Figure 2.7 Schematic of side-loaded specimen compression test ................... 20

Figure 2.8 Sandwich edgewise compression test ...................................... 23

Figure 2.9 Sandwich beam compression test ............................................. 24

Figure 2.10 Hydrostatic compression test ................................................ 25

Figure 2.11 Flexural test. (a) Three-point bending test. (b) Four-point bending

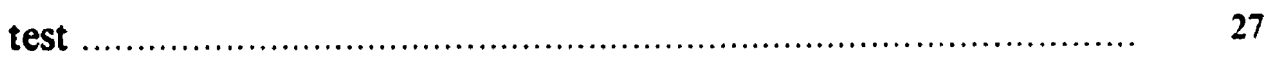

Figure 2.12 Distribution of the longitudinal stress through the thickness of a beam subjected to three-point bending at mid-span $\left(E_{\mathrm{xx}} / G_{\mathrm{xz}}=50\right.$,

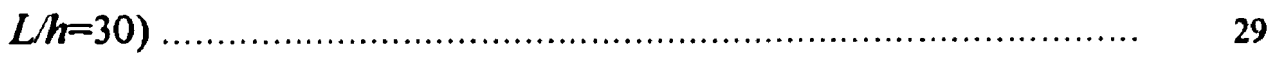

Figure 2.13 Test configuration for (a) two-rail and (b) three-rail shear tests ...... 31

Figure $2.14 \quad 10^{\circ}$ off-axis tensile test ..................................................... 34

Figure $2.15 \quad \pm 45^{\circ}$ tensile test ............................................................ 36

Figure 2.16 Iosipescu shear test .......................................................... 38

Figure 2.17 Notched-specimen test method ............................................... 43

Figure 3.1 Geometry of simply supported beam for analytical solution .......... 49

Figure 3.2 Simply supported beam subjected to harmonic loading on both its 
surfaces

Figure 3.3 Idealization of a beam subjected to three-point bending ................ 53

Figure 3.4 Geometry of the beam used for the numerical investigation ............ 59

Figure 3.5 The effect of load magnitude on mid-span deflections for $r=5 \mathrm{~mm}$, $E_{z:}=10 \mathrm{GPa}$ and $v_{x z}=0.25$. (a) Non-normalized results. (b)

Normalized results

Figure 3.6 The effect of rollers radius on mid-span deflections for $E_{z:=10}$

GPa and $v_{x=}=0.25$. (a) Non-normalized results. (b) Normalized results

Figure 3.7 The effect of $E_{z z}$ on the mid-span deflections for $r=5 \mathrm{~mm}$ and $v_{x z}=$ 0.25 . (a) Non-normalized results. (b) Normalized results

Figure 3.8 The effect of $v_{x}=0.25$ on the mid-span deflections for $r=5 \mathrm{~mm}$ and $E_{z:}=10 \mathrm{GPa}$. (a) Non-normalized results. (b) Normalized results

Figure 4.1 Simply supported beam subjected to harmonic loading

Figure 4.2 Distribution of the displacement in $\mathrm{z}$ direction over the thickness of the beam in exact elasticity solution

Figure 4.3 Comparison of the deflections of the TTTI and the exact elasticity solution

Figure 4.4 Comparison of the stresses obtained from the TTTI and the exact elasticity solution for a beam with $L / h=4$. (a) Longitudinal stresses. (b) Shear stresses ......................................................... 73

Figure 4.5 Configuration of the beams subjected to a concentrated load ......... 74

Figure 4.6 Comparison of mid-span deflections determined from the TTTI and the exact elasticity solutions

Figure 4.7 Comparison of deflections determined from the TTTI and the exact elasticity solutions for beam with $L / h=4$

Figure 4.8 Comparison of stresses determined from the TTTI and the exact 
elasticity solutions for beam with $L / h=4$

Figure 4.9 Distribution of the through-the-thickness normal stress at various level of a bearn with $L / h=4$

Figure 4.10 Comparison of the stresses obtained from the TTTI and the exact elasticity solutions. (a) Longitudinal stresses. (b) Shear stresses ....

Figure 5.1 Representation of the mid-span concentrated load and the reactions with uniformly distributed load

Figure 5.2 Graphic representation of Eqn (5.8). (a) For specimens with $L / h=$

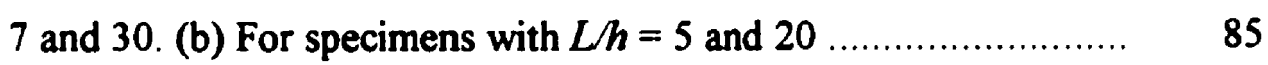

Figure 5.3 Schematic view of the characteristic line .................................... 88

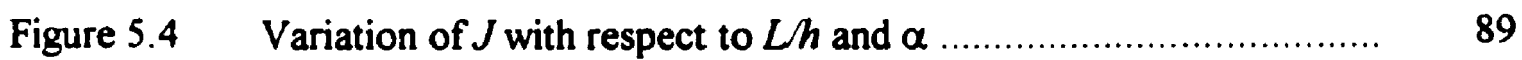

Figure 5.5 Flowchart for the VSM based on the Timoshenko beam theory ..... 90

Figure 6.1 Variation of the shear effect in three-point bending ...................... 92

Figure 6.2 Free body diagram of beam under three-point bending when the supports are not friction-free

Figure 6.3 Effect of support friction on the net mid-span deflection. (a) For material type I. (b) For material type II ........................................ 97

Figure 6.4 Two possible alignments for the VSM .................................... 98

Figure 6.5 Load-displacement curves. (a) From the elasticity analysis. (b) From flexural test on a graphite/epoxy specimen with $L / h \cong 5.5 \ldots .$.

Figure 6.6 Finite element three dimensional model

Figure 6.7 Variation of $\alpha$ for long specimens $(7 \leq L / h \leq 30)$ with $b / h=1$ and $G_{13} / G_{23}=1$

Figure 6.8 Variation of $\alpha$ for short specimens $(5 \leq L / h \leq 20)$ with $b / h=1$ and $G_{13} / G_{23}=1$

Figure 6.9 Variation of $\alpha$ for long specimens $(7 \leq L / h \leq 30)$ with $b / h=3$ and $G_{13} / G_{23}=1$

Figure 6.10 Variation of $\alpha$ for short specimens $(5 \leq L / h \leq 20)$ with $b / h=3$ and 
$G_{13} / G_{23}=1$

Figure 6.11 Variation of $\alpha$ for long specimens $(7 \leq L / h \leq 30)$ with $b / h=5$ and $G_{13} / G_{23}=1$

Figure 6.12 Variation of $\alpha$ for short specimens $(5 \leq L / h \leq 20)$ with $b / h=5$ and $G_{13} / G_{23}=1$

Figure 6.13 Variation of $\alpha$ for long specimens $(7 \leq L / h \leq 30)$ with $b / h=1$ and $G_{13} / G_{23}=2$

Figure 6.14 Variation of $\alpha$ for short specimens $(5 \leq L / h \leq 20)$ with $b / h=1$ and $G_{13} / G_{23}=2$

Figure 6.15 Variation of $\alpha$ for long specimens $(7 \leq L / h \leq 30)$ with $b / h=3$ and $G_{13} / G_{23}=2$

Figure 6.16 Variation of $\alpha$ for short specimens $(5 \leq L / h \leq 20)$ with $b / h=3$ and $G_{13} / G_{23}=2$

Figure 6.17 Variation of $\alpha$ for long specimens $(7 \leq L / h \leq 30)$ with $b / h=5$ and $G_{13} / G_{23}=2$

Figure 6.18 Variation of $\alpha$ for short specimens $(5 \leq L / h \leq 20)$ with $b / h=5$ and $G_{13} / G_{23}=2$

Figure 6.19 Characteristic lines of the materials identified in Table 6.1

Figure 7.1 Plot of $1 / E^{\prime}$ versus $J(h / L)^{2}$ and the material characteristic line for GR-1 graphite/epoxy specimens

Figure 7.2 Plot of $1 / E^{\prime}$ versus $J(h / L)^{2}$ and the material characteristic line for GR-2 graphite/epoxy specimens

Figure 7.3 Plot of $1 / E^{\prime}$ versus $J(h / L)^{2}$ and the material characteristic line for GR-3 graphite/epoxy specimens

Figure 7.4 Plot of $1 / E^{\prime}$ versus $J(h / L)^{2}$ and the material characteristic line for GR-4 graphite/epoxy specimens

Figure 7.5 Plot of $1 / E^{\prime}$ versus $J(h / L)^{2}$ for all graphite/epoxy sets 
Figure 7.6 Plot of $1 / E^{\prime}$ versus $J(h \cdot / L)^{2}$ and the material characteristic line for K-1 Kevalr/epoxy specimens

Figure 7.7 Plot of $1 / E^{\prime}$ versus $J(h / L)^{2}$ and the material characteristic line for K-2 Kevalr/epoxy specimens

Figure 7.8 Plot of $1 / E^{\prime}$ versus $J(h / L)^{2}$ and the material characteristic line for K-3 Kevalr/epoxy specimens

Figure 7.9 Plot of $1 / E^{\prime}$ versus $J(h / L)^{2}$ and the material characteristic line for K-4 Kevalr/epoxy specimens

Figure 7.10 Plot of $1 / E^{\prime}$ versus $J(h / L)^{2}$ and the material characteristic line for K-5 Kevalr/epoxy specimens

Figure 7.11 Plot of $1 / E^{\prime}$ versus $J(h / L)^{2}$ and the material characteristic line for K-6 Kevalr/epoxy specimens

Figure 7.12 The scatter plot of $1 / E^{\prime}$ versus $J(h / L)^{2}$ for all sets of Kevlar/epoxy specimens

Figure 7.13 Plot of $1 / E^{\prime}$ versus $J(h / L)^{2}$ and the material characteristic line for GL-1 E-glass/epoxy specimens

Figure 7.14 Plot of $1 / E^{\prime}$ versus $J(h / L)^{2}$ and the material characteristic line for GL-2 E-glass/epoxy specimens

Figure 7.15 Plot of $1 / E^{\prime}$ versus $J(h / L)^{2}$ and the material characteristic line for GL-3 E-glass/epoxy specimens

Figure 7.16 Plot of $1 / E^{\prime}$ versus $J(h / L)^{2}$ and the material characteristic line for GL-4 E-glass/epoxy specimens

Figure 7.17 Plot of $1 / E^{\prime}$ versus $J(h / L)^{2}$ for all sets of E-glass/epoxy specimens

Figure H.1 The outline of the apparatus used for the VSM 212

Figure H.2 The VSM apparatus installed in MTS testing machine 213

Figure H.3 E-glass/epoxy specimen in the VSM apparatus 214 
Figure H.4 Front view of the VSM apparatus

Figure J.1 Load-deflection curves obtained for graphite/epoxy GR-1 specimens. Top: First loading. Bottom: Second loading

Figure J.2 Load-deflection curves obtained for graphite/epoxy GR-2 specimens. Top: First loading. Bottom: Second loading

Figure J.3 Load-deflection curves obtained for graphite/epoxy GR-3 specimens. Top: First loading. Bottom: Second loading

Figure J.4 Load-deflection curves obtained for graphite/epoxy GR-4 specimens. Top: First loading. Bottom: Second loading

Figure J.5 Load-deflection curves obtained for Kevlar/epoxy K-1 specimens. Top: First loading. Bottom: Second loading

Figure J.6 Load-deflection curves obtained for Kevlar/epoxy K-2 specimens. Top: First loading. Bottom: Second loading

Figure J.7 Load-deflection curves obtained for Kevlar/epoxy K-3 specimens.

Top: First loading. Bottom: Second loading

Figure J.8 Load-deflection curves obtained for Kevlar/epoxy K-4 specimens.

Top: First loading. Bottom: Second loading

Figure J.9 Load-deflection curves obtained for Kevlar/epoxy K-5 specimens.

Top: First loading. Bottom: Second loading

Figure J.10 Load-deflection curves obtained for Kevlar/epoxy K-6 specimens.

Top: First loading. Bottom: Second loading

Figure J.11 Load-deflection curves obtained for E-glass/epoxy GL-1

specimens. Top: First loading. Bottom: Second loading

Figure J.12 Load-deflection curves obtained for E-glass/epoxy GL-2 specimens. Top: First loading. Bottom: Second loading

Figure J. 13 Load-deflection curves obtained for E-glass/epoxy GL-3 specimens. Top: First loading. Bottom: Second loading

Figure J.14 Load-deflection curves obtained for E-glass/epoxy GL-4 
specimens. Top: First loading. Bottom: Second loading 


\section{LIST OF SYMBOLS AND ABBREVIATIONS}

\begin{tabular}{|c|c|}
\hline$a, d, e$ & quantities defined by Eqn (3.1) \\
\hline$a_{n}$ & the $m$ th coefficient of Fourier series for load on beam \\
\hline$a_{n}^{b}$ & the $n$th coefficient of Fourier series for load on bottom surface of beam \\
\hline$a_{n}^{t}$ & the $n$th coefficient of Fourier series for load on top surface of beam \\
\hline$A$ & area of section \\
\hline$A_{\mathrm{i}}$ & unknown coefficients in Eqns (3.7), (3.8) and (4.27) \\
\hline$b$ & width of section \\
\hline $\bar{b}$ & half width of mid-span load \\
\hline$B$ & $\begin{array}{l}\text { function defining the distribution of shear force through the thickness of } \\
\text { beam }\end{array}$ \\
\hline$\hat{B}$ & Laplace transformation of $B$ \\
\hline $\bar{c}$ & $\begin{array}{l}\text { half width of reaction force in simply supported beam under three-point } \\
\text { bending }\end{array}$ \\
\hline E & modulus of elasticity \\
\hline$E_{\mathbf{a}}$ & modulus of elasticity under standard strain rate \\
\hline$E_{11}, E_{22}, E_{33}$ & moduli of elasticity in the principle directions of material \\
\hline$E^{\prime}$ & apparent modulus of elasticity defined by Eqns (5.13) and (5.18) \\
\hline$F$ & applied force \\
\hline$G$ & shear modulus \\
\hline$G_{\mathbf{s t}}$ & shear modulus under standard strain rate \\
\hline$G_{13}, G_{23}, G_{33}$ & shear modulus in material coordinates system \\
\hline$h$ & depth of section \\
\hline$H$ & horizontal reaction force of beam support \\
\hline$I$ & moment of inertia of section \\
\hline$J$ & coefficient defined by Eqns (5.15) and (5.19) \\
\hline$K$ & $=F / \Delta$, flexural stiffness \\
\hline
\end{tabular}


span of simply supported beam

$L^{\prime}$

total length of beam under three-point bending

$m_{i}$

quantity defined by Eqn (3.9)

$n, m$

positive integer numbers

$p$

$=\frac{\pi n}{L}$

$=\frac{\pi n}{L^{\prime}}$

distributed load on beam

$q_{b}$

load distributed on bottom surface of beam

$q$,

load distributed on top surface of beam

$Q_{n}$

coefficient of Fourier series for horizontal reaction forces

$[\bar{Q}]$

stiffness matrix in material coordinate system

stiffiness matrix in $x y z$ coordinate system

$r$

$r_{m}$

$r_{1}$

s

$S_{\mathrm{i} j}$

[S]

$[\bar{S}]$

$t$

$\left[T_{\varepsilon}\right]$

$\left[T_{\sigma}\right]$

$u, v, w$

V

$w_{\mathbf{0}}$

$x, y, z$ radius of loading nose and/or support rollers

radius of loading nose

radius of support rollers

variable in the Laplace transformation domain

components of compliance matrix

compliance matrix in material coordinate system

compliance matrix in $x y z$ coordinate system

thickness

strain transformation matrix

stress transformation matrix

components of displacement in $x y z$ coordinate system

shear force of section

amplitude of deflection for sinusoidal load

coordinate axes 
distance measured from the center of beam support

material principle axes

$\alpha$

$=\frac{\bar{b}}{h}$

quantity defined by Eqn (4.13)

$\delta_{1}, \delta_{2}$

quantities defined by Eqn (3.15)

deflection of beam at mid-span

$\Delta_{\mathrm{L}}, \Delta_{\mathrm{N}}, \Delta_{\mathrm{SN}}, \Delta_{\mathrm{T}}$ quantities defined by Eqn (3.30)

$\Delta_{\text {gecoural }}$

deflection due to bending

$\Delta_{\text {shear }}$

deflection due to shear

$\Delta_{0}$

mid-span deflection of beam with friction-free supports

$\varepsilon$ normal strain

$\varepsilon_{g 1}, \varepsilon_{g 2}, \varepsilon_{g}$

strains from three strain gages attached to the $10^{\circ}$ off-axis shear test

$\varepsilon_{\mathrm{xx}}, \varepsilon_{\mathrm{yy}}, \varepsilon_{\mathrm{zz}}$ components of normal strain in $x y z$ coordinate system

$\varepsilon_{11}, \varepsilon_{22}, \varepsilon_{33}$ components of normal strain in material coordinate system

$\varepsilon_{45}, \varepsilon_{-45}$ strains from two strain gages attached to the Iosipescu specimen

$\dot{\varepsilon}$ normal strain rate

$\dot{\varepsilon}_{\max }$ strain rate at the outermost layer of simply supported beam $\dot{\boldsymbol{\varepsilon}}_{s t}$ normal strain rate for standard test condition

$\{\varepsilon\}$ vector of strains in material coordinate system vector of strains in $x y z$ coordinate system shear strain

$\gamma_{x z}, \gamma_{y z}, \gamma_{x y} \quad$ components of shear strain in $x y z$ coordinate system

$\gamma_{13}, \gamma_{23}, \gamma_{12} \quad$ components of shear strain in material coordinate system shear strain rate average shear strain rate in section

$\dot{\gamma}_{\text {sf }}$ shear strain rate for standard test condition 


$\begin{array}{ll}\eta & \text { quantity defined by Eqn (4.21) } \\ \mu & \text { friction coefficient } \\ v_{13}, v_{23}, v_{12} & \text { Poisson's ratios in material principle directions } \\ \phi & =\sqrt{E / G} \\ \sigma & \text { normal stress } \\ \sigma_{x x}, \sigma_{y y}, \sigma_{z z} & \text { components of normal stress in xyz coordinate system } \\ \sigma_{11}, \sigma_{22}, \sigma_{33} & \text { components of normal stress in material coordinate system } \\ \{\sigma\} & \text { vector of stress in material coordinate system } \\ \{\bar{\sigma}\} & \text { vector of stress in } x y z \text { coordinate system } \\ \tau & \text { shear stress } \\ \tau_{x z}, \tau_{y z}, \tau_{x y} & \text { components of shear stress in } x y z \text { coordinate system } \\ \tau_{13}, \tau_{23}, \tau_{12} & \text { components of shear stress in material coordinate system } \\ \theta & \text { rotation angle of axis } / \text { with respect to } x \text { axis as shown in Fig. } 1.1 \\ \text { FRPC } & \text { fiber-reinforced plastic composite } \\ \text { IITRI } & \text { Illinois Institute of Technology Research Institute } \\ \text { TTTI } & \begin{array}{l}\text { through-the-thickness inextensibility } \\ \text { VSM }\end{array}\end{array}$




\section{ACKNOWLEDGEMENTS}

In his name who is the most knowledgeable. This thesis has been completed due to the assistance and support of many people. I would like to take this opportunity to thank all of them; those with whom I was in direct contact, and those who assisted me indirectly.

I gratefully acknowledge the Ministry of Culture and Higher Education of Iran for providing me with a scholarship for pursuing my Ph.D. at DalTech. I am also very thankful for the University of Mazandaran, Iran, and in particular its president Dr. A. Sheikholeslami for allowing me to undertake a Ph.D. program abroad while remaining a faculty member of the university.

Special thanks and acknowledgements are due to my supervisor, Dr. F. Taheri for his continuous support on all sides. While providing both technical and financial supports, he has always been a friend to me. Thanks to his efforts and those of Professor $\mathrm{H}$. Vaziri, the head of the Civil Engineering Department, I had the pleasure of working as a part-time faculty member in the Department while working on my Ph.D. program. I express my gratitude to my guiding committee, Dr. G. Fenton and Dr. T.S. Koko, and my external examiner Dr. S.V. Hoa for their technical guidance and reviewing my work.

The staff of DalTech were tremendous help to me. In particular, I would like to thank Mr. R. Sarty, Mr. B. Nickerson, and Mr. A. Macpherson for their assistance in the fabrication of my test apparatus and in the preparation of the test specimens. I would also like to acknowledge the Advanced Material Engineering Center (AMEC), Halifax, NS for providing me with the use of their facilities and raw materials. Special thanks goes to Mr. Mike Pineo for his assistance. TW Pultrusion Ltd., Dartmouth, NS and Glasform Inc. San Jose, CA are also acknowledged for providing me with materials. 
I wish to thank my wife, Mehri, for producing an environment which enabled me to focus my efforts on completing this thesis. I should also thank my son, Pedrum, for his patience and co-operation, for whom I could not spend enough time, when he needed it the most. I thank my mother, my brothers and sisters who have always been my encouragement for my further education and a great help to me. Finally, I express my respect and appreciation to my father whose presence is now sadly missed. 


\begin{abstract}
The longitudinal and the shear moduli of fiber-reinforced plastic composites (FRPC) are commonly measured by separate test methods. This thesis introduces a new test method which enables one to measure these properties simultaneously. In the new test method, specimens with different span-to-depth ratios $(L / h)$ are subjected to three-point bending. The method will be called the "Varying-Span Method" (VSM), since for a given material producing different $L / h$ is simply achieved by changing the test span.
\end{abstract}

One of the common methods for evaluating the longitudinal modulus of FRPC is to conduct flexural tests on rectangular specimens. The longitudinal modulus is determined by a simple equation which is based on the Euler beam theory. Since this theory does not account for the shear deformation, the evaluated modulus is usually underestimated. The effect of shear deformation reduces when $L h$ of the specimen increases. However, since in general FRPC have low shear modulus, to eliminate the shear deformation effect from the evaluated modulus, one must use specimens with large span to depth ratios (say $L h>60$, see for example, Zweben et.al. 1979). In the VSM, instead of eliminating the effect of the shear deformation from the result of the flexural test, the attribute is used to simultaneously evaluate both the longitudinal and the shear moduli of the material. In this method, the test is considered as a phenomenon with two unknowns, namely, the longitudinal and the shear moduli. Theoretically, these unknowns can be obtained by conducting at least two tests on specimens with different $L / h$.

Among the advantages of the VSM is its capability of measuring the through-the-thickness shear modulus of specimens with relatively small thickness (say $2 \mathrm{~mm}$ ). For this, a special apparatus was designed and fabricated. The apparatus was used for the evaluation of the longitudinal and shear moduli of composites made of graphite/epoxy, Kevlar/epoxy and E- 
glass/epoxy. The results and their comparison with the values obtained by other common test methods are presented.

A review of the most popular methods for the measurement of the longitudinal and the shear moduli of FRPC is presented in this manuscript. The advantages and the disadvantages of each method are discussed. The exact elasticity solution of a beam subjected to three-point bending is developed. This solution is used as a means to assess the integrity of the VSM. The relevant theories with respect to the VSM are reviewed. The application of these theories is discussed and a solution which accounts for the local effect of the concentrated load and the reaction forces on the deformation of the beam is presented. Factors that influence the accuracy and efficiency of the proposed method are identified and investigated. The integrity of the method is examined and proved by theoretical and experimental investigations. 


\section{Chapter 1}

\section{INTRODUCTION}

Commercially available filamentary materials such as glass, carbon, boron and Kevlar fibers provide a combination of high strength, high modulus and low specific gravity. These fibers, combined with a matrix, produce materials with superior properties in comparison to conventional metallic materials. The matrix material may be a polymer, a metal, or a ceramic. In general, the function of matrix is to keep the fibers in the desired location and orientation, to transfer the loads between the fibers, and to protect the fibers from environmentally induced damages, while the fibers act as the principal load-carrying elements. The materials produced in this way are called "fiber-reinforced composites". Comprehensive discussion on mechanics, design and manufacturing of these types of materials can be found in Mallick (1993), Gibson (1994), Tsai (1988) and Schwartz (1984).

In this thesis, attention is focused on fiber-reinforced polymeric composites (FRPC) in which the polymeric materials constitute the matrix. FRPC are the most common type of composite materials produced and used in industries. Metal and ceramic metal matrix composites are comparatively new and do not yet have a large data base. However, since there is no major difference between the mechanics of FRPC and the mechanics of metal and ceramic matrix composites, most of the discussions and the solutions presented in this thesis are applicable to these composites as well.

\subsection{Nomenclatures}

In structural applications, FRPC are used in several forms. The most common form is called laminate. A laminate is obtained by stacking a number of thin layers composed of fibers and matrix. These layers are also called plies or laminae. The fiber orientations and the stacking sequences can be controlled to obtain the desired physical and mechanical 
properties for the composite laminate in different directions. Consequently, the product has an anisotropic nature, making the design and analysis process considerably more difficult and complicated than that of structures made up of isotropic materials such as metals.

The behavior of a laminated structure can be defined when the properties of its layers are known. With some exceptions, the layers can be defined as orthotropic material with nine independent elastic constants. These constants are defined according to each layer's principal axes. Figure 1.1 shows the definition of these axes for a fiber-reinforced lamina. As shown in this figure, axis $I$ is along the fiber length and represents the longitudinal direction of the lamina. Axes 2 and 3 represent the transverse in-plane and through-thethickness directions, respectively. The exception occurs when fibers with different orientations form a layer. In this case, it is convenient to assume each fiber direction is located in a separate layer. Consequently, the assumption of orthotropy will not be violated.

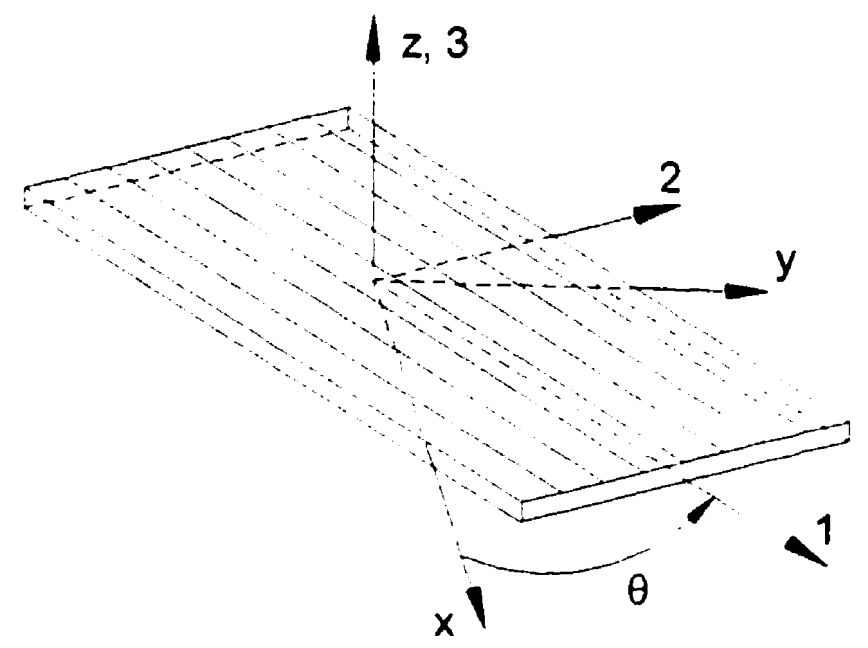

Figure 1.1 Definition of material principal axes and loading axes for a fiber-reinforced lamina. 
The elastic constants relate the stress components to the corresponding strain components produced in a body due to external and/or internal loads. To define these components, the stress and strain nomenclature shown in Fig. 1.2 is adopted throughout the thesis. Subscript $x, y$ and $z$ may be replaced by 1,2 and 3 , however in this case, the reference coordinate system will be the principal axes defined in figure 1.1. It is important to note that only one $x y z$ coordinate system is defined for a laminate, while each layer has its own local 123 coordinate system. The stress components identified by $\sigma$ are the normal stresses, while those denoted by $\tau$ are the shear stresses. Strains correspond to these stresses are denoted by $\varepsilon$ and $\gamma$, respectively. The stress-strain relations are usually presented in a matrix form. These relations in local coordinate system can be represented by,

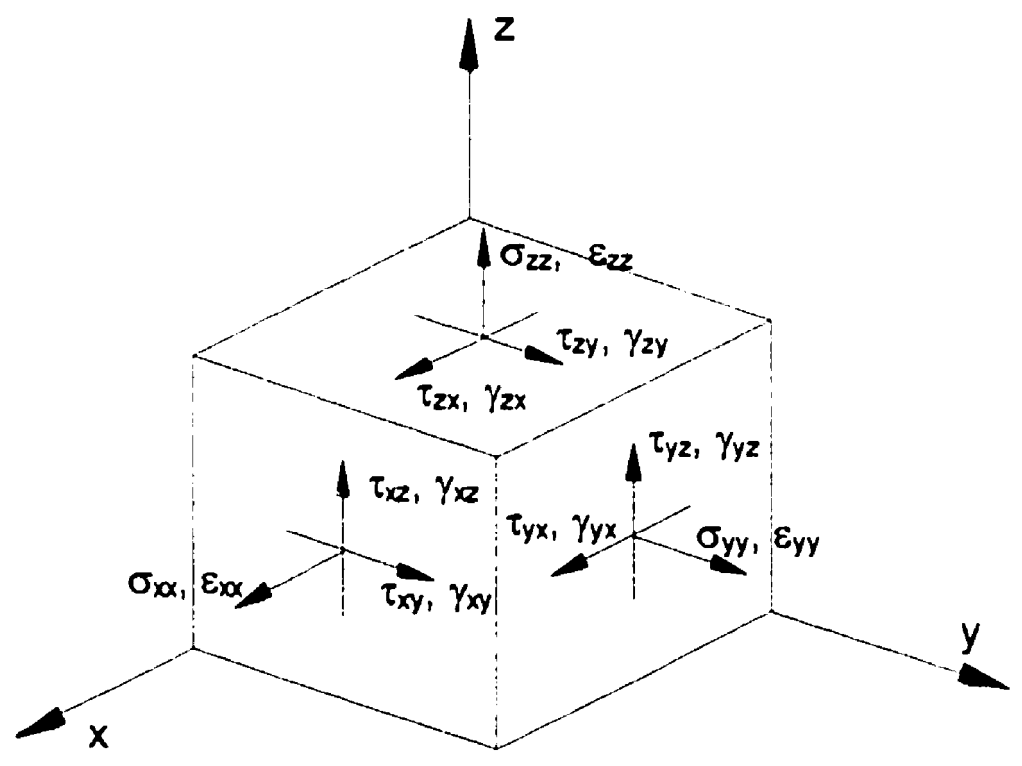

Figure 1.2 Stress and strain nomenclature 


$$
\left\{\begin{array}{l}
\varepsilon_{11} \\
\varepsilon_{22} \\
\varepsilon_{33} \\
\gamma_{23} \\
\gamma_{13} \\
\gamma_{12}
\end{array}\right\}=\left[\begin{array}{cccccc}
\frac{1}{E_{11}} & \frac{-v_{12}}{E_{11}} & \frac{-v_{13}}{E_{11}} & 0 & 0 & 0 \\
\frac{-v_{12}}{E_{11}} & \frac{1}{E_{22}} & \frac{-v_{23}}{E_{22}} & 0 & 0 & 0 \\
\frac{-v_{13}}{E_{11}} & \frac{-v_{23}}{E_{22}} & \frac{1}{E_{33}} & 0 & 0 & 0 \\
0 & 0 & 0 & \frac{1}{G_{23}} & 0 & 0 \\
0 & 0 & 0 & 0 & \frac{1}{G_{13}} & 0 \\
0 & 0 & 0 & 0 & 0 & \frac{1}{G_{12}}
\end{array}\right]\left\{\begin{array}{l}
\sigma_{11} \\
\sigma_{22} \\
\sigma_{33} \\
\tau_{13} \\
\tau_{12} \\
\tau_{12}
\end{array}\right\}
$$

or when written in compact form:

$$
\{\varepsilon\}=[S]\{\sigma\}
$$

The inverse of Eqn (1.2) is

$$
\{\sigma\}=[S]^{-1}\{\varepsilon\}=[Q]\{\varepsilon\}
$$

where $\{\varepsilon\},\{\sigma\},[S]$ and $[Q]$ are the strain vector, stress vector, the compliance matrix and the stiffness matrix in the local coordinate system of 123 , respectively. $E, \cup$ and $G$ represent the elastic modulus, Poisson's ratio and the shear modulus in a particular principal direction denoted by their subscripts, respectively. These quantities are known as the elastic constants of the material and are defined by Eqns (1.4) through (1.6) when the stresses except in the specified direction are zero.

$$
\begin{gathered}
E_{11}=\frac{\sigma_{11}}{\varepsilon_{11}} \quad E_{22}=\frac{\sigma_{22}}{\varepsilon_{22}} \quad E_{33}=\frac{\sigma_{33}}{\varepsilon_{33}} \\
\mathrm{v}_{12}=\frac{\varepsilon_{22}}{\varepsilon_{11}} \quad \mathrm{v}_{13}=\frac{\varepsilon_{33}}{\varepsilon_{11}} \quad \mathrm{v}_{23}=\frac{\varepsilon_{33}}{\varepsilon_{32}} \\
G_{12}=\frac{\tau_{12}}{\gamma_{12}} \quad G_{13}=\frac{\tau_{13}}{\gamma_{13}} \quad G_{23}=\frac{\tau_{23}}{\gamma_{23}}
\end{gathered}
$$

The stress-strain relations in the $x y z$ coordinate system have the following forms

$$
\{\bar{\varepsilon}\}=[\bar{S}]\{\bar{\sigma}\}
$$




$$
\{\bar{\sigma}\}=[S]^{-1}\{\bar{\varepsilon}\}=[\bar{Q}]\{\bar{\varepsilon}\}
$$

where $\{\bar{\sigma}\}$ and $\{\bar{\varepsilon}\}$ are the stress and strain vectors, and $[\bar{S}]$ and $[\bar{Q}]$ are the compliance and the stiffness matrices in the $x y z$ coordinate system, respectively. $[S]$ and $[\bar{Q}]$ are related to $[S]$ and $[Q]$ through the following relations

$$
[S]=\left[T_{\sigma}\right]^{T}[S]\left[T_{\sigma}\right]
$$

and

$$
[\bar{Q}]=\left[T_{\varepsilon}\right]^{T}[Q]\left[T_{\varepsilon}\right]
$$

where $\left[T_{\sigma}\right]$ and $\left[T_{c}\right]$ are the stress and the strain transformation matrices, respectively. It is common to define the $x y z$ coordinate system in such a way that the $z$ axis is parallel to the axis 3 of the plies. In this case the transformation matrices take the following forms.

$$
\begin{aligned}
& {\left[T_{\sigma}\right]=\left[\begin{array}{cccccc}
\cos ^{2} \theta & \sin ^{2} \theta & 0 & 0 & 0 & 2 \cos \theta \sin \theta \\
\sin ^{2} \theta & \cos ^{2} \theta & 0 & 0 & 0 & -2 \cos \theta \sin \theta \\
0 & 0 & 1 & 0 & 0 & 0 \\
0 & 0 & 0 & \cos \theta & -\sin \theta & 0 \\
0 & 0 & 0 & \sin \theta & \cos \theta & 0 \\
-\cos \theta \sin \theta & \cos \theta \sin \theta & 0 & 0 & 0 & \cos ^{2} \theta-\sin ^{2} \theta
\end{array}\right]} \\
& {\left[T_{c}\right]=\left[\begin{array}{cccccc}
\cos ^{2} \theta & \sin ^{2} \theta & 0 & 0 & 0 & \cos \theta \sin \theta \\
\sin ^{2} \theta & \cos ^{2} \theta & 0 & 0 & 0 & -\cos \theta \sin \theta \\
0 & 0 & 1 & 0 & 0 & 0 \\
0 & 0 & 0 & \cos \theta & -\sin \theta & 0 \\
0 & 0 & 0 & \sin \theta & \cos \theta & 0 \\
-2 \cos \theta \sin \theta & 2 \cos \theta \sin \theta & 0 & 0 & 0 & \cos ^{2} \theta-\sin ^{2} \theta
\end{array}\right]}
\end{aligned}
$$

where $\theta$, as shown in Fig. 1.1 , is the angle between the $x$ axis and the $l$ axis. For a more detailed and comprehensive discussion on the subject, and the proof of the above equations the reader is referred to Whitney (1987). 
In laminated structures, the thickness of the laminate with respect to its other dimensions is usually small. Therefore, it is common to assume that the normal stress in the throughthe-thickness direction of the laminate $\left(\sigma_{z=}\right)$ in comparison to the other stress components is negligible. A special case of laminated structures is when the structure behaves as a beam. The coordinate system for laminated beam is shown in Fig 1.3. In this case, the dimensions of the beam in the $y$ and $z$ directions are small, and therefore, it is admissible to neglect the stress components in these directions, i.e. normal stresses $\sigma_{y y}$ and $\sigma_{z:}$, and the shear stresses $\tau_{x y}$ and $\tau_{y y}$. Notice that the assumption of $\tau_{x y}=0$ is valid only when there is no lateral load in the $y$ direction. Substituting zero values for $\sigma_{y y}, \sigma_{z z}, \tau_{x y}$ and $\tau_{y=}$ in Eqn (1.7), one obtains the following stress-strain relations.

$$
\begin{gathered}
\sigma_{x x}=E_{x x} \varepsilon_{x x} \\
\tau_{x}=G_{x x} \gamma_{x}
\end{gathered}
$$

where

$$
\begin{gathered}
E_{x x}=\left[\cos ^{4} \theta S_{11}+\cos ^{2} \theta \sin ^{2} \theta\left(2 S_{12}+S_{66}\right)+\sin ^{4} \theta S_{22}\right]^{-1} \\
G_{x=}=\cos ^{2} \theta G_{13}+\sin ^{2} \theta G_{23}
\end{gathered}
$$

$S_{11}, S_{22}, S_{12}$ and $S_{66}$ are the members of the compliance matrix of Eqn (1.1). $E_{x \mathrm{x}}$ and $G_{x z}$ are also called the longitudinal and the through-the-thickness shear moduli of the layer, respectively. For $\theta=0^{\circ}$ and $\theta=90^{\circ}, E_{x x}$ and $G_{x=}$ will become

For $\theta=0^{\circ}$ :

$$
\begin{aligned}
& E_{x x}=E_{11} \\
& G_{x e}=G_{13}
\end{aligned}
$$

For $\theta=90^{\circ}$ :

$$
\begin{aligned}
& E_{x x}=E_{12} \\
& G_{x=}=G_{23}
\end{aligned}
$$




\subsection{Evaluation of the Elastic Constants}

An accurate evaluation of the elastic constants defined in Eqns (1.4) through (1.6) is necessary for the correct simulation and prediction of the behavior of FRPC structures. Several test methods have been developed to evaluate these properties. Some of these methods have gained more popularity because either they have easy procedures and/or they use specimens with simple geometry. A review of the most common test methods are presented in the next chapter. The advantages and disadvantages of each of them are also briefly discussed.

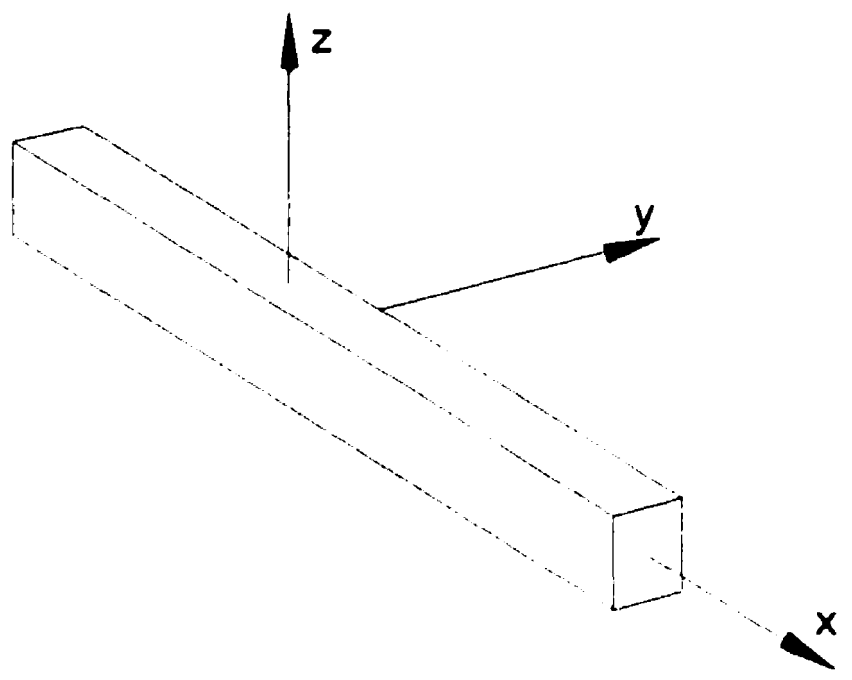

Figure 1.3 The Coordinates system in beam type problem.

Among these methods, three- and four- point bending tests are unique due to several inherent properties. These methods use a simple fixture to host specimens with simple rectangular geometry, and they do not require additional machining or tabbing. No strain gages or other expensive instrumentation or grips are required either. However, since most FRPC have low shear moduli, the evaluated longitudinal modulus by these methods depend on the span-to-depth ratio $(L / h)$ of the specimens. This characteristic of FRPC is used as the basis for the development of the method introduced by Jalali and Taheri (1997, 1998d), and is presented in this thesis in more detail. The method can simultaneously 
evaluate the longitudinal and the shear moduli of FRPC. The new method is called the "varying-span method" (VSM). This is due to the usage of specimens with different spans $(L)$ for producing different $L / h$.

In the VSM, unidirectional specimens (usually $0^{\circ}$ or $90^{\circ}$ ) are subjected to three-point bending. The flexural stiffness of each specimen is obtained from the load versus net midspan deflection curve recorded during the test. The stiffness of the specimen is a function of the longitudinal and the shear moduli of its material. The shear modulus has significant effect when $L / h$ of the specimen is small. The effect of the shear modulus rapidly decreases as $L / h$ increases. On the other hand, the longitudinal modulus has the dominant effect when $L / h$ is large. This characteristic enables one to evaluate both the longitudinal modulus and the shear modulus of the material by subjecting at least two specimens with different $L / h$ (one with a small $L / h$ and one with a large $L / h$ ) to three-point bending. However, for higher accuracy and reliability one should test more than two specimens.

\subsection{Applicable Theories for the VSM}

The behavior of a beam subjected to three-point bending can be predicted by different theories. Among these theories the Euler beam theory is the simplest one. It assumes that the plane normal to the centriodal axis of the beam before deformation remains plane and normal to the axis after deformation. This theory has been used extensively in structural applications. For details and the applications of this theory one may refer for example to text books by Timoshenko and Young (1965), Ghali and Neville (1978) and Smith (1988). The Euler theory ignores the deformation due to shear. Nevertheless, it gives fairly accurate results for most structural applications. This is due to the fact that in most structural members made of traditional materials such as steel, concrete and wood, the components' span-to-depth ratios are sufficiently large so that the shear deformation effect is quite negligible. For example for a simply supported concrete beam with rectangular cross section the shear deformation is responsible for about $2 \%$ of the total deformation of 
the beam when $L / h=10$ and the load is distributed uniformly along the entire length of the beam. It should be noted that in most cases $L / h$ of structural members made of traditional materials is larger than 10 .

The longitudinal modulus is the only elastic constant that one requires for the Euler beam theory. This theory, when applied to the data obtained from a three-point bending test will provide one with the flexural modulus of the material. The flexural modulus obtained this way is very similar to the longitudinal modulus when a relatively large $L / h$ specimen is used. However, one cannot evaluate the shear modulus of the material using this theory.

The exact elasticity solution is another method of predicting the behavior of a three-point bending beam. A comprehensive discussion on elasticity solutions for isotropic materials is given by Timoshenko and Goodier (1970) and for anisotropic materials, by Lekhnitskii (1981). A solution for an orthotropic beam under concentrated load(s) is given by Whitney (1985). A more accurate solution, however, for an orthotropic beam under threepoint bending will be presented in Chapter 3 of this manuscript. These solutions provide exact results when the assumed boundary conditions are compatible with the actual boundary conditions of the beam. They are usually used for validating other solutions which, because of their simplifying assumption(s), are approximate in nature. However, the major problem with the elasticity solutions is that they provide solutions for only simple cases. Besides that, they are often too complicated for every day applications. Also, elasticity solutions depend on elastic constants other than the longitudinal and the through-the-thickness shear moduli. This, coupled with the complexity of the elasticity solution, make them unsuitable for use with the VSM.

A suitable theory to form the basis of the VSM is one that includes the longitudinal modulus and the through-the-thickness modulus of the material as the only elastic constants for the accurate prediction of the three-point bending beam's deflections. For 
this, the Timoshenko beam theory and the through-the-thickness inextensibility theory are two theories that can efficiently be implemented for the VSM.

In the Timoshenko beam theory, it is assumed that planes normal to the centriodal axis of the beam before deformation remain plane after deformation. But in contrast to the Euler beam theory, the planes do not remain normal to the centriodal axis after deformation. The theory determines the deflections due to shear forces in addition to that caused by bending. Timoshenko (1983) acknowledges Poncelet as the first person who considered the effect of shear deflection for beams. However, the theory in its present form was introduced by Timoshenko (1921 and 1922). Others, for instance Gere (1963) and Cowper (1966) had also substantial contributions to the application of the theory. In this theory, the deflection of beams are composed of two separate parts, the deformation caused by bending and the deformation caused by shear.

The through-the-thickness inextensibility theory was developed for laminated plates by Jalali and Taheri (1998a, b, c). In this theory, deflection of plates along the thickness is assumed to be constant. This assumption, in conjunction with the equilibrium and the stress-strain relationships, leads to at least one differential equation. The solution of the differential equation(s) depends on the boundary conditions of the laminates, and as in the case of the elasticity solutions, provides answers only for simple cases. One of the advantages of the through-the-thickness inextensibility solution in comparison with the elasticity solutions is that it requires a smaller number of elastic constants. In beam type problems, the through-the-thickness inextensibility solution depends only on the longitudinal and the through-the-thickness shear moduli. Therefore, one can effectively apply the theory to the VSM. The solution for three-point beam based on this theory is given in Chapter 4. 
While the Timoshenko and the through-the-thickness inextensibility theories provide accurate results for the overall deflections, they are not capable of predicting the local deformations due to concentrated load(s) and the reaction forces at supports. As a result, to obtain accurate results from the VSM, one must be able to measure the overall deflection of the specimen. In order to achieve this requirement, a special apparatus was designed and fabricated in-house. The description of the apparatus is presented in Appendix $\mathrm{H}$. It was used for all the VSM tests conducted for the compilation of the data for the thesis.

\subsection{Organization of the Thesis}

Chapter 2 of this manuscript presents a review of the common test methods used for the evaluation of the elastic constants of FRPC. The test methods are critically evaluated and several aspects, such as the economy, the practicality and the reliability are used for their comparison. Chapter 3 presents the elasticity solution of a orthotropic beam under threepoint bending. The solution is used as a means for the evaluation of the integrity of the VSM. Chapters 4 and 5 are dedicated to the development of the VSM based on the through-the-thickness inextensibility and the Timoshenko beam theories, respectively. The theoretical investigation to evaluate the integrity of the VSM is presented in Chapter 6. Chapter 7 presents the experimental investigations. In this chapter the longitudinal and the shear moduli of several FRPC are evaluated by the VSM and the comparison is made with the results obtained by other common test methods. Summary and the conclusion of the work are presented in Chapter 8 . Computer programs written and used in the thesis and the description of the VSM apparatus along with many complimentary data are provided in the appendices. 


\section{Chapter 2}

\section{AVAILABLE TEST METHODS REVIEW}

Design and analysis of FRPC structures require one to know the stress-strain relationship of the constituent laminates in their principal directions, defined in Fig. 1.1. In general, the stress-strain relationships of materials may follow a nonlinear trend. However, in engineering applications, it is common to define the stress-strain relationship by two parameters, that is, the elastic modulus and the strength. As shown in Fig. 2.1, the elastic modulus may be defined as the slope of the initial tangent to the stress-strain curve, however, it is more common to take the slope of a secant line or a chord line for elastic modulus. The definition of the strength is also shown in Fig. 2.1. In some cases the strength is defined as the stress corresponding to a specified strain.

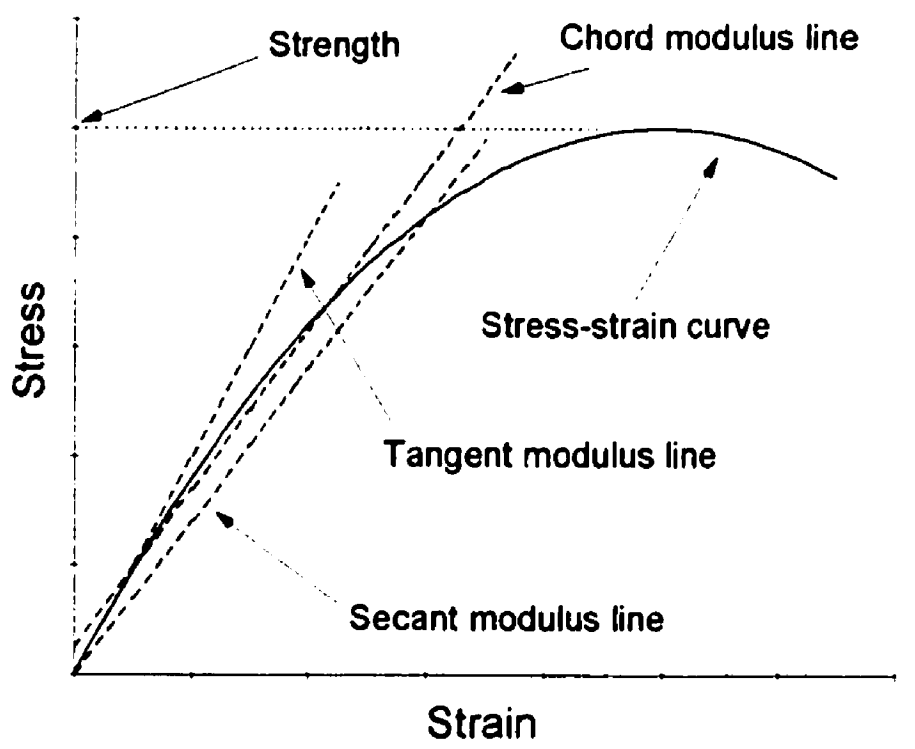

Figure 2.1 Definition of various elastic moduli and the strength of materials.

The stress-strain diagrams for all reinforcing fibers in use are linear up to the point of failure, therefore the tangent, the secant and the chord moduli are identical. However, for 
polymeric solids, the stress-strain diagrams show nonlinear behavior. The shape of the diagrams also change by the variation of loading rate and ambient temperature. In FRPC, the matrix plays a minor role when the material is subjected to tensile load along the longitudinal direction of fibers (axis $I$ ), therefore the stress-strain diagram like that of the constituent fibers is linear and shows a brittle failure. However, the behavior of FRPC when subjected to shear stresses is matrix dominant and exhibits a nonlinear stress-strain relationship.

The evaluation of different moduli and the corresponding strengths of FRPC are the scope of several test methods available in the literature. In this chapter we review some of these methods. However, our effort will be focused on those that have gained more popularity, and are commonly used. Furthermore, we restrict ourselves to those methods that measure the properties that are evaluated by the VSM. These tests can be classified into four categories: tension, compression, flexural and shear tests. The advantages and disadvantages of each test will be discussed. A short review on the latest achievements in this field is also presented.

\subsection{Tension tests}

The evaluation of longitudinal moduli along the material principal axes $I$ and 2 ( $E_{11}$ and $E_{22}$ ) and the corresponding strengths can be determined by tension tests. The various test methods are discussed in below.

\subsubsection{Strip-specimen tension test (ASTM D3039-93)}

With this method a thin flat strip of material having a constant rectangular cross-section is subjected to tension (Fig. 2.2) while the longitudinal and transverse strains, and the applied load are simultaneously measured and recorded. The longitudinal and transverse strains are measured in mid-length of the specimen using strain gages and/or extensometers. The specimen must have sufficient length so that the measured strains 
would be free of the stress concentration regions due to the test grips. Tabs are attached to the two ends of the specimen, to prevent the premature failure of the specimen in the grip zones and to promote a mid-length failure.

The test result usually includes the effect of bending moment caused by the misalignment of the grips, or the specimen itself. Misalignment of the specimen occurs when the specimen is gripped improperly or when it is out of tolerance. Excessive bending causes premature failure of the specimen and highly inaccurate modulus. ASTM E1012-93 provides a guideline for the evaluation of the bending and describes the potential sources of such misalignment. The degree of bending in a tensile system can also be evaluated by using the procedure described in ASTM D3039-93. A system with less than $3 \%$ bending is considered to be a good testing practice. When bending is greater than $3 \%$, the average longitudinal strain should be used for the evaluation of the elastic modulus. In such a case, one must attach two strain gages/extensometers on both faces of the specimen, in the longitudinal direction.

The method provides $E_{11}$ and the corresponding tensile strength of the material when unidirectional $0^{\circ}$ specimens are used. Test on $90^{\circ}$ specimens provides $E_{22}$ and the tensile strength in this direction. The test method and the geometry of the specimen are simple; however, the need for tabs and the misalignment problem are considered as major shortfalls of this method. In addition, when testing thick $0^{\circ}$ specimens, one often requires a high capacity testing machine and expensive hydraulically operated grips.

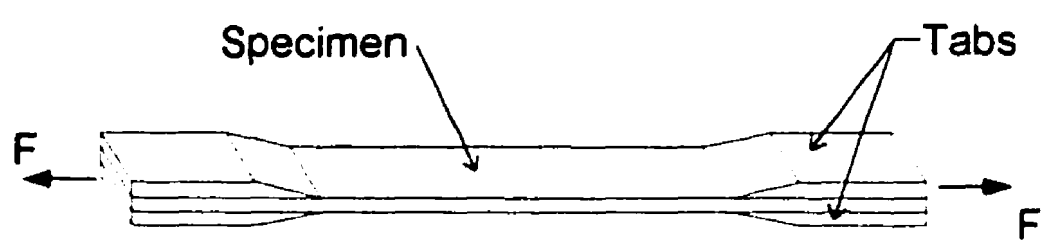

Figure 2.2 ASTM D3039-93 tensile test. 


\subsubsection{Dogbone-specimen tension test (ASTM D638M-93)}

A dogbone specimen is shown in Fig. 2.3. It has a flat surface and uniform thickness. The gradual increase in the cross section of the specimen at two ends are to prevent the premature failure of the specimen due to stress concentration in the grip zones. The test procedure is the same as outlined in the ASTM D3039 tension test, except for the fact that the specimen needs no tabs.

The elimination of tabs in a dogbone specimen reduces one source of misalignment. However, producing the dogbone shape requires precise machining which makes the test expensive and time consuming. Machining of composites made of carbon and graphite fibers should be done using special precautions since smoke and fumes produced during cutting are very hazardous. Machining of Kevlar composites by conventional methods using carbide blades or end mills induces delaminations and fuzzy edges. Therefore, special cutting devices, such as laser or water jet cutting techniques, may be necessary. The method is good for neat resin, however when it is used for FRPC, machining of the specimen is the biggest problem.

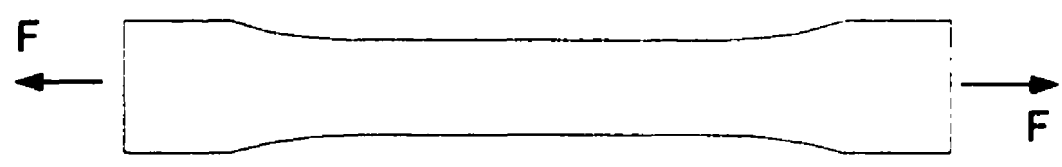

Figure 2.3 A typical dogbone specimen for tension test.

\subsubsection{Hydrostatic tension test}

In this method, a ring type specimen of approximately $25 \mathrm{~mm}$ diameter machined from a thin-wall composite cylinder is subjected to internal hydrostatic pressure. The procedure for fabrication of the thin-wall composite cylinder for ring-type specimen is outlined in ASTM D2291-83. The specimen is instrumented from outside by attaching strain gages along the longitudinal and hoop directions. For the specifics of hydrostatic tension test 
setup, one may refer to Daniel and Ishai (1994). Munjal et. al. (1983) reported a good correlation between the data obtained from tension tests on flat coupons and ring specimens. Ring specimens are more representative of cylindrical filament-wound components as compared to flat coupons. However, preparation of ring specimens is time consuming and costly. Moreover, the need for strain gages and special fixtures has made the method less attractive.

\subsubsection{Split disk method (ASTM D2290-92)}

The test setup for split disk method is shown in Fig. 2.4. As the figure shows, a ring-type specimen is loaded under tension through the two split disks fitted inside the specimen. The tensile stress in the specimen is calculated from the following equation

$$
\sigma=\frac{F}{2 t b}
$$

where $t$ and $b$ are the thickness and the width of the ring, respectively. However, the test specimen experiences bending moment; as a result, the evaluated tensile modulus and tensile strength cannot represent the true properties of the material. Test data obtained from this method are recommended only for material evaluation and quality control [Munjal (1989)].

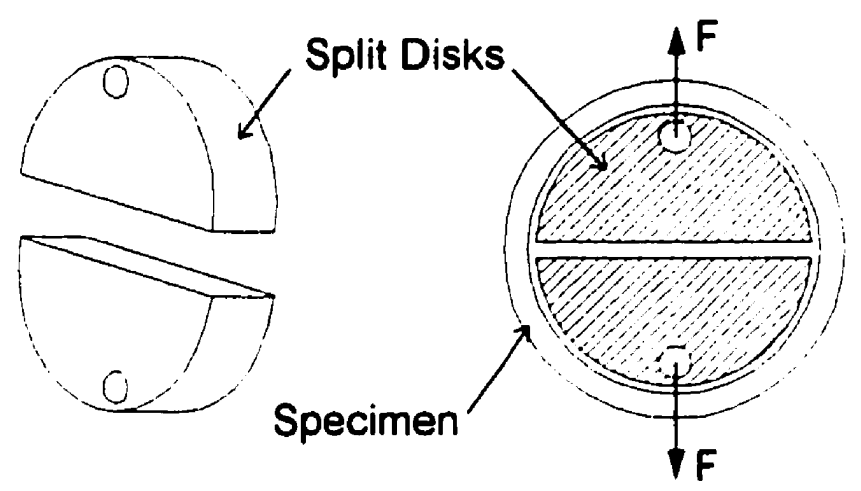

Figure 2.4 Test setup for split disk method. 
To reduce the effect of the bending moment during the test, Chiao and Hamstand (1976) and Clements and Chiao (1977) used elongated ring specimens. Clements and Chiao (1977) compared the results of elongated ring specimens with those of flat panels specimens. They obtained lower strength but higher modulus for the elongated ring. Scatter of the data obtained for elongated ring specimens was also higher.

The fixture for the split disk method is simple. However, the preparation of the specimen is time consuming and expensive. Moreover, the data obtained from this method is not acceptable for design purposes. Nevertheless, the method provides a convenient way for the quality control of tubular components, such as pressure pipes.

\subsubsection{Filament-wound pressure vessel tension test (ASTM D2585-68)}

In this test, a filament-wound pressure vessel with internal diameter of $146 \mathrm{~mm}$ is subjected to internal hydrostatic pressure (Fig. 2.5). For the evaluation of elastic modulus, strain gages are bonded to the outside surface of the vessel. Internal pressure is increased gradually while the strains and pressure are recorded simultaneously. The method provides the tensile strength and modulus of FRPC; however, the quantities obtained should not be considered as the true properties of the material, because a) the material is subjected to biaxial tension, and b) the fibers are not unidirectional. The latter phenomenon, as was shown by Feldman et. al. (1966) and Pagano and Whitney (1970), produces a complex behavior that makes the interpretation of the test results a complicated task. ASTM D2585 calls the measured quantities "apparent properties" of FRPC. The method is good for the evaluation of the material and the process of filament winding and curing. It is also used for the purpose of quality control and acceptance or rejection of the actual product's manufacturing specifications. 


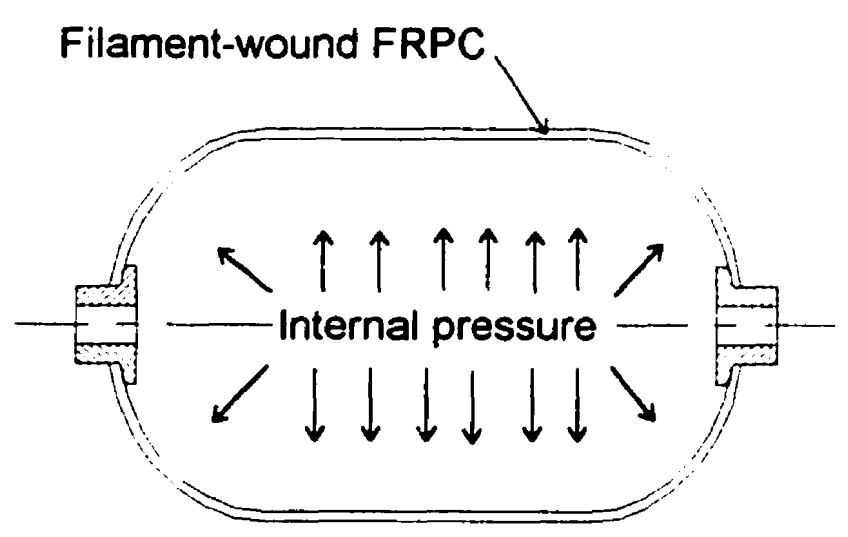

Figure 2.5 Filament-wound pressure vessel.

\subsection{Compression tests}

Unlike ductile metals, the compressive strength and modulus of $0^{\circ}$ FRPC are not equal to their tensile strength and modulus. Among the commercially used fibers, FRPC made of Kevlar have considerably lower strength and modulus in compression than in tension. Carbon and Glass FRPC show slightly lower strength and modulus under compression than in tension, while there is virtually no difference between the tensile and compressive properties of boron FRPC (See for example, Piggott and Harris (1980) and Mallick (1993)). The compressive properties of FRPC are difficult to obtain because of the tendency for premature failure due to buckling and end brooming. A number of test methods incorporating a variety of specimen designs and loading fixtures have been developed to overcome the associated problems with the compression tests. A review on these methods was presented by Whitney et. al. (1985). Adsit (1983) and Lamothe and Nunes (1983) showed that the values of compressive modulus are generally independent of the test method. Huwever, the compressive strength depends on mode of failure which varies from one test method to another. If the failure mode is not truly compressive, low compressive strength will be obtained. In general, a good test method induces a fiber compression failure, giving a high compressive strength value. The more commonly used compression test methods are reviewed in this section. 


\subsubsection{End-loaded specimen (ASTM 695-91)}

In this test method, a flat strip specimen with uniform thickness is placed in the jig shown in Fig. 2-6. The nuts and screws on the jig are finger-tightened, so that the specimen can slide freely inside. The specimen is then loaded at its two ends. The compressive strength data obtained from this method on $0^{\circ}$ specimen is consistently on the low side because of premature failure due to end brooming. Despite the lateral support, there is also the possibility of having a buckling mode of failure. In order to prevent the premature failure due to end brooming, a dogbone specimen (Fig. 2.1) may be used. However, as stated earlier, the preparation of this type specimen is not easy and is costly. Moreover, the data obtained from tests on the dogbone specimens does not indicate considerable improvement over those obtained from the strip specimens. Munjal (1989) attributes this problem to the stress concentration present at the corners of the dogbone specimens which causes premature failure. In the investigation undertaken by the ASTM Committee D-30, Adsit (1983) reports that end-loaded specimens seem to fail by some form of delamination or shear. He does not recommend the method for FRPC.

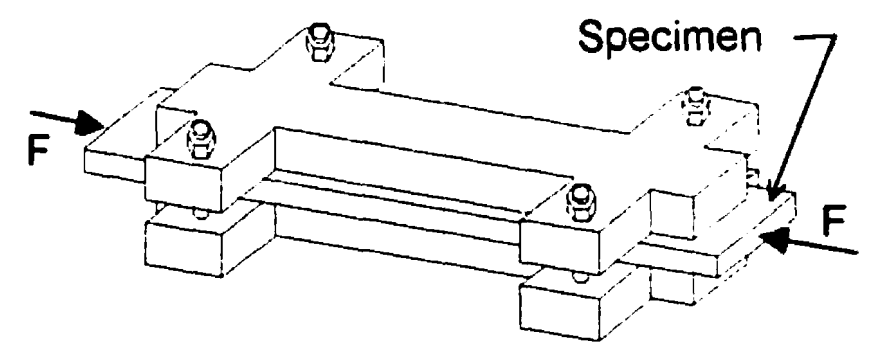

Figure 2.6 ASTM 695-91 fixture for FRPC compression test.

\subsubsection{Side-loaded specimen (ASTM D3410-95)}

A flat strip of material having a constant rectangular cross-section is used for the test. The specimen, like the one shown in Fig. 2.2, is tabbed at two ends, but longer tabs are used to leave only a short length of the specimen unsupported. The compression force is induced in the specimen by shear action between the tabs and the specimen. Two types of fixtures 
are commonly used for this purpose. In both fixtures, the load is transferred to the tabs by a shear load acting along the grips via wedge action between the tapered grips and the tapered sleeves, Fig. 2.7. The difference between the two fixtures is that in one fixture the wedges are conical and in the other one the wedges are trapezoidal. The former fixture is known as the Celanese fixture. The latter one, which was first developed at the Illinois Institute of Technology Research Institute, is known as the IITRI fixture. The compression tests using the two fixtures are referred to as Procedure A and Procedure B, respectively, by the ASTM D3410-95 standard.

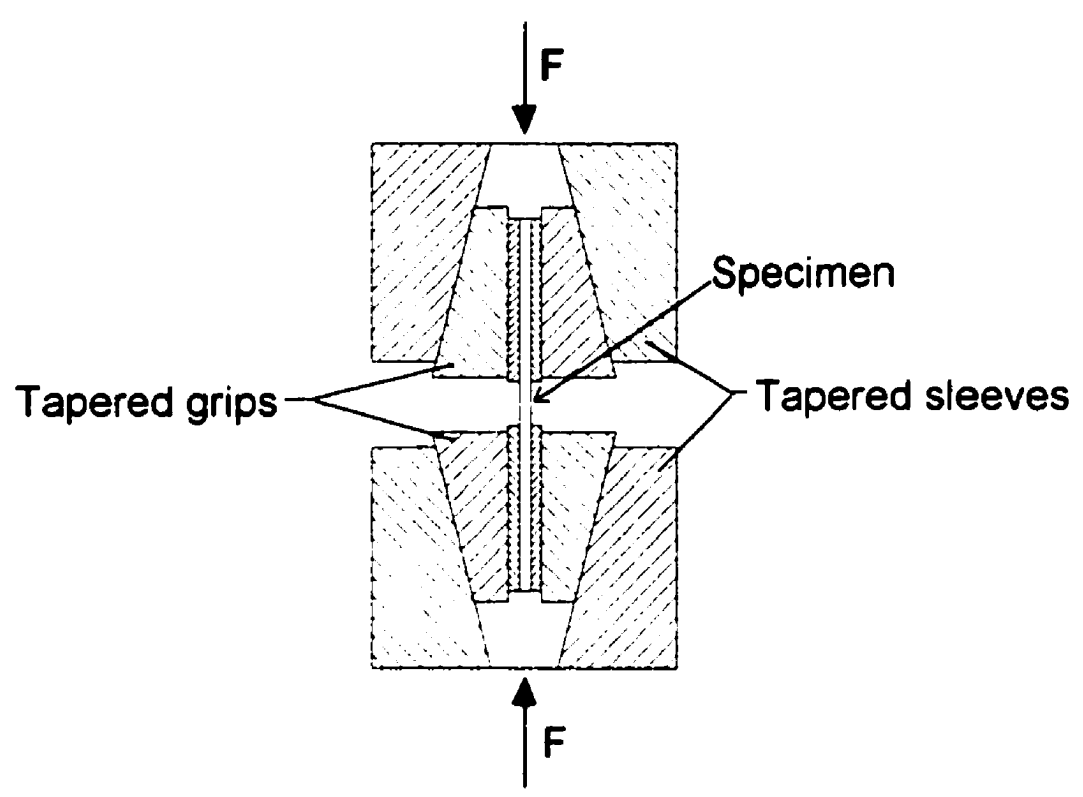

Figure 2.7 Schematic of side-loaded specimen compression test.

The conical wedges from Procedure $A$ are known to be prone to cone-to-cone seating problem. Mechanically, the Celanese fixture grips do not seat properly on a cone-to-cone surface contact arrangement. Instead, contact occurs along a pair of lines on the opposite sides of the specimen, at each end of the specimen. This unstable condition causes a lateral shift in the grips, which in turn, produces high frictional forces in the enveloping cylinder. This situation can result in erroneously high values for the compressive strength and 
modulus. This problem was discussed by Hofer and Rao (1977). The trapezoidal wedge grips in IITRI fixture eliminates the problem of line contact, but the fixture is much bigger in size and weight than the Celanese fixture.

The data resulting from both the Celanese and the IITRI fixtures are sensitive to the flatness and parallelism of the tabs, as well as the whole system alignment. Therefore, special care must be taken to assure that the specimen tolerance requirements are met. This usually requires precision grinding of the tab surfaces after bonding them to the specimen, which makes the test expensive and time consuming. System with poor alignment, as was discussed in Sec. 2.1.1, produces bending moment in the specimen which in turn, results in premature failure and erroneous modulus. Every effort should be made to eliminate bending from the test system. Nevertheless, the experimental work done by Adams and Odom (1991) did not show satisfactory results with this test method. They studied the effect of the type of tabbing material and tab taper on the measured axial compressive strength using the IITRI fixture. In part of their work, they report:

"Nearly every specimen of all the configurations tested failed in a region in close proximity to the ends of the tabs, rather than randomly throughout the gage section. Therefore, a true axial compressive strength of the composite, i.e., a strength independent of the configuration, was apparently not being measured. That is, the present results suggest that tabbing material and geometry need to be studied further in the research for an optimum specimen configuration.

In summary, compression testing of highly orthotropic, high strength composite materials, e.g., unidirectional-reinforced carbonepoxy composites, is very sensitive to specimen geometry and test technique. Existing standards are sufficiently general that less than satisfactory results can be obtained even if all guidelines are fully conformed to." 
The work done by Sinclair and Chamis (1983) also did not show that a unique failure mode was associated with the compression failure of the specimens tested using the IITRI fixture.

The specimen unsupported length must be short enough to prevent buckling. On the other hand, this region must be long enough to allow stress decay to uniaxial compression and to minimize the Poisson restraint effects due to the grips. This subject was discussed in more detail by Bogetti et. al. (1988), and Adams and Lewis (1991).

Despite the problem stated by Adams and Odom (1991), use of the IITRI fixture for the evaluation of compression properties is very common in industry. When compared with more accurate methods, the IITRI method is less expensive and simpler. The work carried out by the ASTM Committee D-30 [Adsit (1983)] showed the method gave test data comparable to those obtained from the sandwich beam test method.

\subsubsection{Sandwich edgewise compression test (ASTM C364-94)}

In this test, a sandwich specimen composed of two composite coupons bonded to an aluminum honeycomb core is subjected to compression, Fig. 2.8. The honeycomb core is to provide lateral stability to the composite coupons. It is also assumed that the core does not carry any load. Compressive load is applied through the end caps. They are for supporting the specimen at its two ends to prevent premature buckling failure due to separation of the facings from the core at the point of contact with the loading plates. The caps also prevent end crushing. The two ends of the specimen must be machined so that they are parallel to each other and at right angles to the length of the specimen. The results of the test are very dependent on the parallelism of the loaded surfaces. The preparation of the specimen is time consuming and expensive. Consequently, the test is not widely used. 


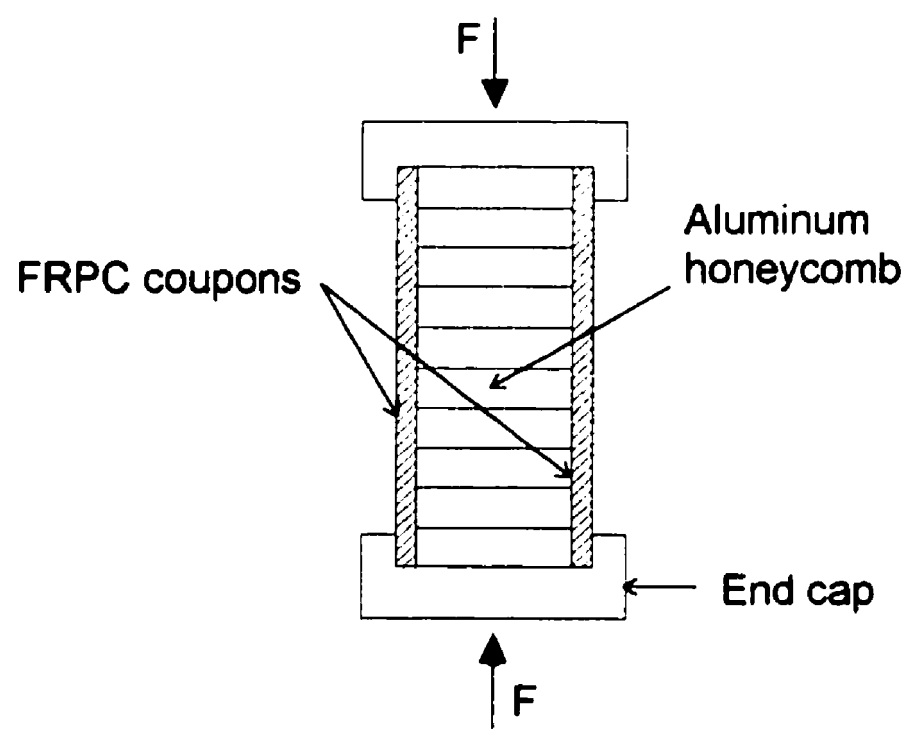

Figure 2.8 Sandwich edgewise compression test.

\subsubsection{Sandwich beam compression test (ASTM D5467-93)}

A four-point flexural test is conducted on a sandwich beam specimen composed of a honeycomb core with a composite sheet bonded on the top and a metal sheet bonded on the bottom side, Fig. 2.9. The top facing sheet undergoes longitudinal compressive stress. The honeycomb is to prevent the top facing from buckling. But its material is so selected that it does not carry considerable load. The thickness of the composite and metal faces must be adjusted to ensure compressive failure in the top face. Longitudinal strain is measured by attaching two strain gages to the top plate along the longitudinal axis of the beam. These strain gages are located between the two concentrated loads and are symmetric with respect to the mid-span of the beam. The readings from the two strain gages should not differ more than $10 \%$. The compression stress in the top plate is calculated by ignoring the load carrying effect of the core.

Theoretical investigation by Whitney (1973) revealed that the free edge effect produced a nonuniform compression stress distribution across the width of the composite facing while accompanied by other in-plane and out-of-plane stresses. However, it seems that the 
above mentioned problem does not have significant effect on the result of the test. The experimental work of Shuart (1981) and Adsit (1983) confirmed the integrity of the method for the evaluation of the compressive strength and modulus of FRPC. Nevertheless, the test is not a popular method due to high cost for the preparation of the sandwich specimens and large amount of material needed. The method is not recommended for determining the in-plane Poisson's ratio $\left(v_{x y}\right)$, since the lateral deformation of the composite facing is influenced by the core deformation. Whitney et. al. (1984) believed that the higher value obtained for Poisson's ratio from a sandwich beam as compared to that from a tensile coupon is probably due to boundary effects or to the presence of transverse curvature in the sandwich beam.

To reduce the cost and the amount of the materials used in the sandwich beam test, Gruber et. al. (1981) proposed the reusable sandwich beam concept. They showed that the data obtained by using the reusable beam of glass/Kevlar hybrid composites were consistent with those obtained from the compression test using the IIRTI fixture. Further investigations are needed to extend the finding for general purposes.

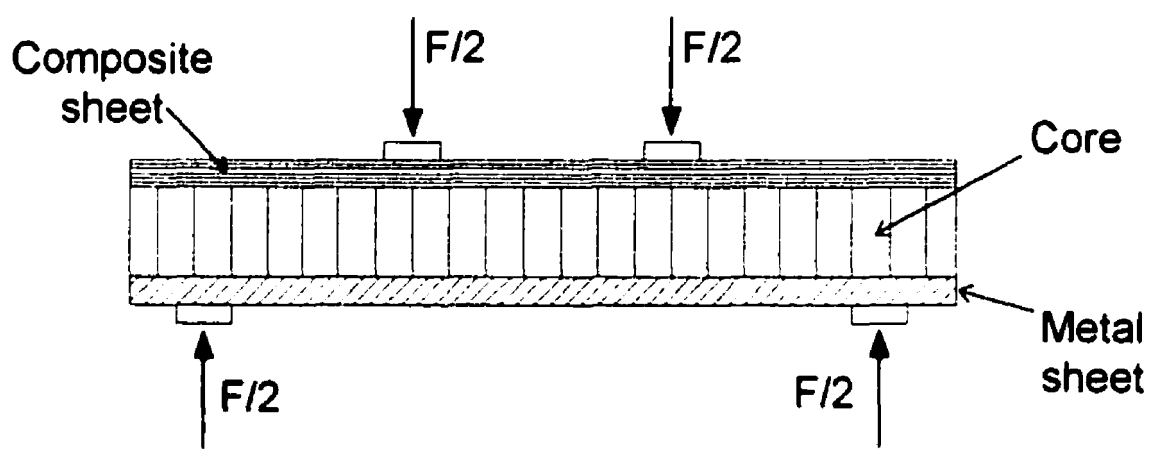

Figure 2.9 Sandwich beam compression test.

\subsubsection{Hydrostatic compression test ( ASTM D2586-68)}

In this test, a cylindrical specimen is plugged at its two ends and is subjected to an external hydrostatic pressure within a compression chamber, Fig 2.10. The test provides the 
compressive strength of the specimen. However, the specimen undergoes a state of biaxial stresses composed of hoop and longitudinal compressive stresses. Therefore the data obtained from this test cannot be considered as the true compressive strength of the laminate. The ASTM D2586 states that the method is limited to constructions containing greater than $50 \%$ by weight of glass reinforcement. The preparation of the test specimen is expensive and requires special manufacturing equipment. However, the compressive strength values obtained from this test provide a convenient means for assessing and comparing the influence of different fiber layouts and resin contents in filament-wound components. The test is also useful for quality control. The values obtained from this method are not recommended for design purposes. ASTM discontinued the test method in 1996.

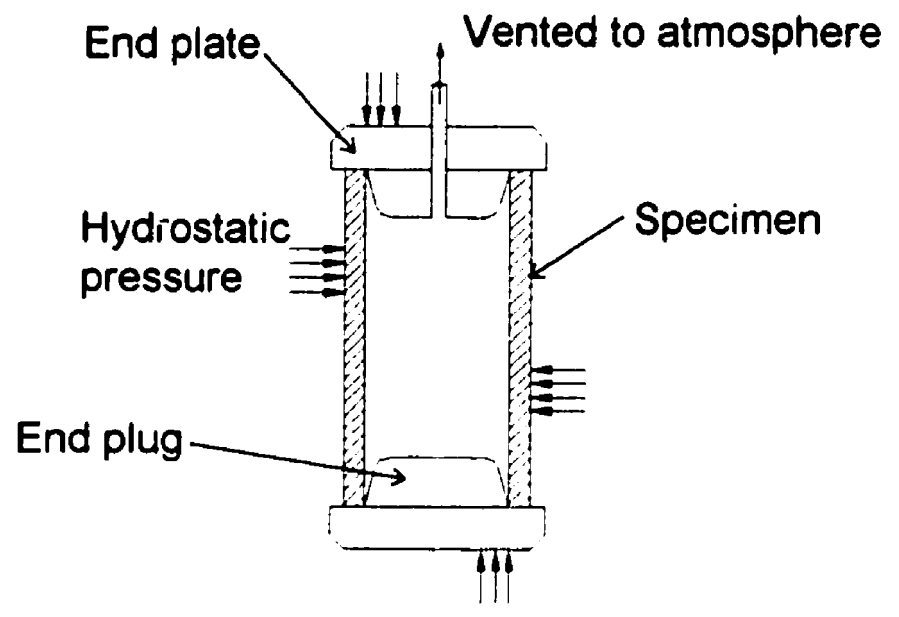

Figure 2.10 Hydrostatic compression test.

\subsection{Flexural test (ASTM D790-93)}

Flexural properties, such as flexural strength and modulus, are determined by test method I or II described in ASTM D790-93. Both methods use a specimen with uniform and rectangular cross-section. In test method $I$, the specimen is loaded in a three-point bending mode, Fig. 2.11 a. In test method II, the specimen is loaded is in four-point bending mode, Fig. $2.1 \mathrm{lb}$. The load is gradually increased while both the load and the deflection of the specimen at mid-span are simultaneously recorded. When the three-point bending test is 
used, the flexural modulus and strength are obtained from the following equations, respectively

$$
\begin{gathered}
E=\frac{F}{\Delta}\left(\frac{L^{3}}{4 b h^{3}}\right) \\
\sigma_{\text {max }}=\frac{3 F_{\max } L}{2 b h^{2}}
\end{gathered}
$$

where, $F / \Delta$ is determined from the slope of the initial straight portion of the loaddeflection curve and $b$ is the width of the specimen. The other parameters are as shown in Fig. 2.11a. When specimens with large span-to-depth ratio (say $L / h>16$ ) are used, significant horizontal reaction forces are developed at the supports which affect the moment in a simply supported beam. In this situation, ASTM 790-93 provides the following equation instead of Eqn (2.3)

$$
\sigma_{\max }=\frac{3 F_{\max } L}{2 b h^{2}}\left[1+6\left(\frac{\Delta}{L}\right)^{2}-4\left(\frac{h}{L}\right)\left(\frac{\Delta}{L}\right)\right]
$$

where $\Delta$ is the deflection of the beam at mid-span corresponding to $F_{\text {max }}$. Similar equations are used to obtain the flexural properties of the material when four-point bending test is used. When unidirectional $0^{\circ}$ or $90^{\circ}$ specimens are tested, the flexural modulus and strength are expected to equate to the corresponding tensile properties. However, in practice different values are obtained from the two tests. This problem will be discussed later.

In comparison to other test methods, flexural tests have many advantages. For example:

a) The test specimen has simple geometry and can be cut directly from the actual component.

b) The possible small curvature and misalignment of the specimen do not influence the test result.

c) Use of strain gage/gages, which are usually costly and time consuming, is not needed.

d) The test fixture is simple. 
e) The flexural test results are less sensitive to the quality of the specimen and its fabrication than the tensile test (see Whitney et. al. (1974)).

Because of these attributes, the flexural test methods have gained special popularity in composite material industry. However, there are some limitations that must be recognized when using the test methods. These limitations are discussed below.

(a)

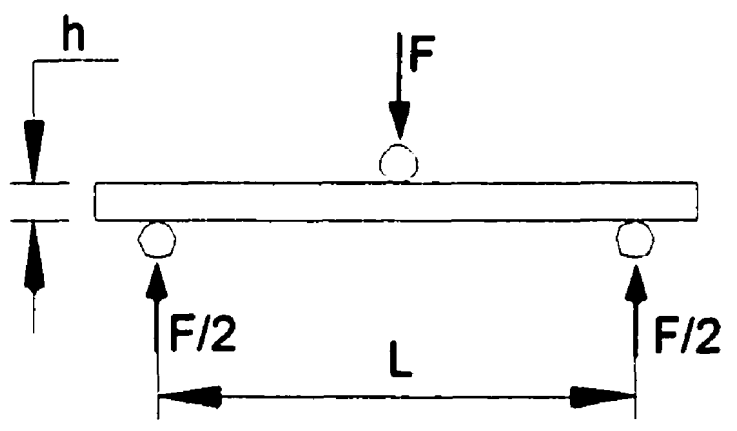

(b)

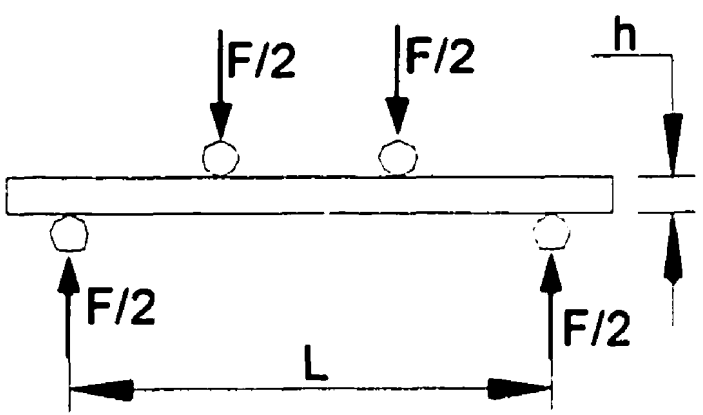

Figure 2.11 Flexural test. (a) Three-point bending test. (b) Four-point bending test.

Some FRPC, as was stated earlier in this chapter, have different response under tension than in compression. Due to this phenomena the stress-strain relationship obtained from flexural test will be different from that obtained from the tensile and compressive tests, since specimens under flexural test undergo a combination of tensile and compressive stresses. On the other hand, the presence of a stress gradient in a flexural test results in higher tensile strength compared to that obtained by tensile test under uniform stress. Owing to the fact that flexural tests are less expensive and time consuming than the tensile/compressive tests, many researchers have tried to correlate the flexural properties to tension/compressive properties. For example, Zhang and Sikarskie (1996) introduced a technique for generating stress-stress curves (for both tension and compression) for composite materials from flexural data. A similar technique was presented by Arai and Oku (1979) for isotropic graphite. Bullock (1974) used a two-parameter Weibull model to correlate the strength data obtained from flexural and tensile tests. His work was limited 
to unidirectional graphite/epoxy composites. A much broader work was carried out by Whitney and Knight (1980). Knight and Hahn (1975) presented similar work for randomly-distributed fiber composites. Although in general, these authors did not find a good correlation with the two-parameter Weibull statistical model, the model nevertheless explained why the flexural strength was expected to be higher than the tensile strength.

Despite the difference between the values obtained from flexural and tensile/compressive tests, the flexural test results can be considered acceptable for design and analysis, because:

a) For design and analysis of FRPC, it is common to define a single value for elastic modulus for both tension and compression in each principal direction. This value is usually defined based on the tensile modulus.

b) For most FRPC the compressive and the tensile moduli do not differ significantly.

c) When determined properly, the flexural modulus correlates very well with the tensile modulus. The experimental results presented in Chapter 7 confirm this fact.

d) The modulus and strength obtained from a flexural test are more representative for structures that are mainly subjected to flexural loading.

A major drawback of the flexural test is the effect of shear deformation in the test results. This problem leads to an underestimated value for flexural modulus. Since FRPC in general have low shear modulus, the deflection measured in flexural test may include a significant amount of shear deformation which is not accounted for in Eqn (2.2). The effect of shear is reduced rapidly when $L h$ of the specimen increases. Zweben et. al. (1979) suggest that $L / h$ greater than 60 be used.

It is also instructive to mention that the validity of Eqns (2.3) and (2.4) are questionable. These equations depend on two phenomena, a) the variation of longitudinal strain $\left(\varepsilon_{\mathrm{xx}}\right)$ through the thickness of the section under consideration must be linear, and b) the material 
must have a linear stress-strain relationship. There are several works in the literature, for example Jalali and Taheri (1998a) and Sandorff (1980), that discuss the effect of concentrated load on the behavior of simply supported orthotropic beams. These works show that the concentrated load changes the distribution of stresses in the vicinity of the load. This phenomenon is illustrated in Fig. 2.12 for a highly orthotropic beam $\left(E_{x x} / G_{x z}=50\right)$ with $L / h=30$. The deviation of the stress from a straight line decreases with increasing $L / h$, and with decreasing orthotropy. The non-linear behavior of the material is usually of minor concern, since in most cases FRPC show a linear behavior up to the failure load or very close to it.

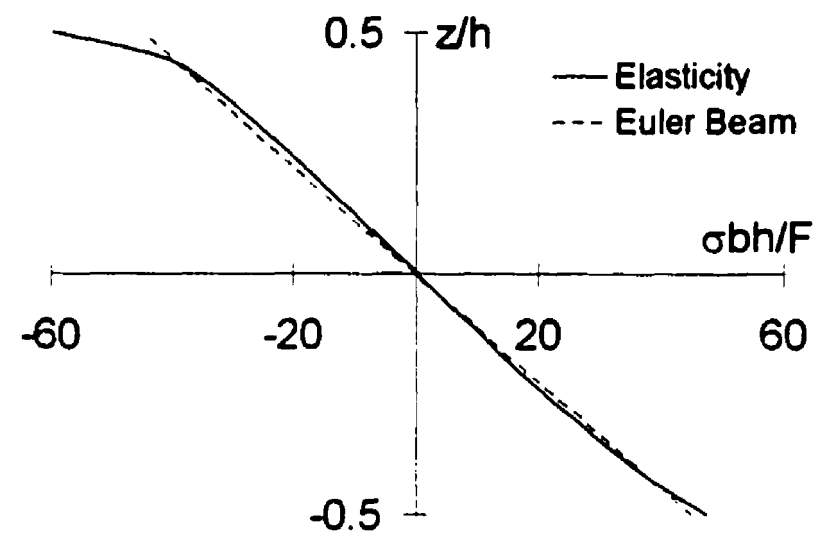

Figure 2.12 Distribution of the longitudinal stress through the thickness of a beam subjected to three-point bending at mid-span $\left(E_{\mathrm{xx}} / G_{\mathrm{xz}}=50, L h=30\right)$.

\section{$2.4 \quad$ In-plane shear tests}

The determination of in-plane shear properties of FRPC has been a controversial subject in composites research and industry. This is due to the fact that producing a pure state of shear stress in a given specimen is not simple. Several test methods are available; however, the results obtained from them do not usually match with each other very well. In this section, we review a few of the most widely used test methods. 


\subsubsection{Torsion test on a tube specimen}

In this test method, a unidirectional thin-wall tube is subjected to pure torsion. The simultaneous recording of the applied torque and the wall strain at $45^{\circ}$ angle with respect to the longitudinal axis enables one to establish the shear stress-strain relationship. Instead of strain gage reading, one may record the relative angular rotation between two points along the length of the tube. Among the several methods available for the evaluation of shear properties of FRPC, the torsion test method is believed to provide the most accurate results. Therefore, it has been used as a mean for the evaluation of the accuracy and validity of other shear test methods [Swanson et. al. (1985), Chiao et. al. (1977) and Sims (1972)]. It is important to note that for tubes with filament wound fibers at angles different from $0^{\circ}$ and $90^{\circ}$, the interpretation of the results obtained from the test is not simple. The laminate will undergo a combination of biaxial and shear stresses. Therefore, three strain gages will be needed to record the strains at three different angles. However, the end constraints will have a significant effect on the results except when a specimen with sufficient length is used. Theoretical discussion of this subject was given by Pagano and Whitney (1970) and Whitney and Halpin (1968). Rizzo and Vicario (1972) also conducted a finite element analysis to evaluate the effect of the grip constraints on the distribution of the stresses in tube specimens. The investigations showed that when $0^{\circ}$ or $90^{\circ}$ specimens are used, accurate data can be obtained using classical shell theory. However, for helically wrapped tubes, the ratios of the wall thickness to diameter and the length to diameter of the tube can influence the determination of the strength and the modulus.

Despite the accuracy of the results obtained from the torsion test on a unidirectional tube specimen, the method is not a cost effective and convenient one. That is, the fabrication of FRPC tubes is an expensive task, requiring special manufacturing machinery. This is in addition to a special apparatus that is required for conducting the test. It is also important to recognize the difference between the fabrication methods of the tube specimen and that 
of the actual structure. Therefore, it is reasonable to expect that the results obtained from the torsion on tube specimens not to be representative of the actual structures. Consequently, in practice other methods that employ flat specimens are used.

\subsubsection{Rail shear tests (ASTM D4255-83)}

ASTM D4255 explains two test methods for the determination of the in-plane shear properties of composite materials, i.e. Method A and Method B. Method A test configuration is shown in Fig. 2.13a. As shown in the figure, two pairs of rails hold the specimen along its sides. Bolts are usually used to fasten the specimen to the rails. This procedure requires drilling holes in the specimen. An in-plane shear force is induced in the specimen by applying a tensile force to the rails. For the evaluation of shear modulus one must attach at least one strain gage in the center of the specimen at $45^{\circ}$ to the longitudinal direction of specimen. However for more accurate results, one may use two to four strain gages. When four strain gages are used, they are mounted in $\pm 45^{\circ}$ configurations to each face.
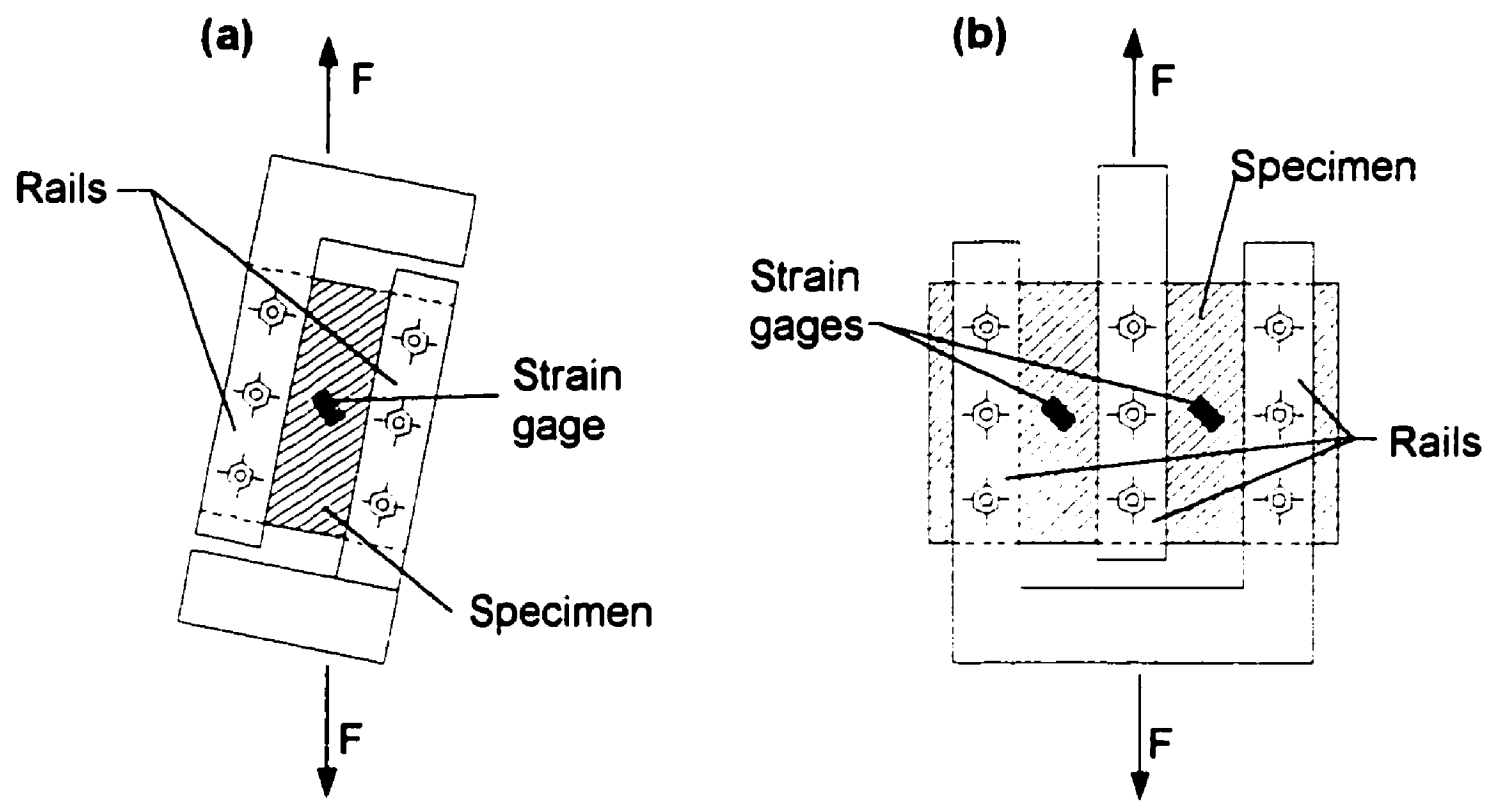

Figure 2.13 Test configuration for (a) two-rail and (b) three-rail shear tests. 
In Method B (Fig. 2.13b) the test fixture consists of three pairs of rails that are fastened to the test specimen. As in Method $A$, the rails and the specimen are usually bolted to each other. The in-plane shear force in the specimen can be produced by either a tensile or a compressive force. Strain gages must be used if shear modulus is to be evaluated.

By simultaneous recording of load and strain, one can obtain the shear stress-strain curve of the specimen. The shear stress and shear strain are related to the applied load and the strain measured at $45^{\circ}$ by the following equations

$$
\begin{aligned}
& \tau=F / A \\
& \gamma=2 \varepsilon_{45}
\end{aligned}
$$

where $A$ is the area of sections resisting the shear force. $\varepsilon_{4 s}$ will be the average strain reading when more than one strain gages are used. Note that since the shear stress at free edges of the specimen must be zero, theoretically, a uniform distribution of shear stress throughout the section is not possible. Therefore, the shear stress calculated from the above equation cannot be the true stress at the center of the specimen where the strain is measured. However, investigations conducted by Whitney et. al. (1971) and Garcia et. al. (1980) showed that the difference between the true value and the value obtained from Eqn (2.5) was not very significant. That is due to the fact that, in general, a uniform state of shear stress can be obtained at a short distance away from the free edges of the specimen.

In the rail-shear tests, the specimens usually fail in an out-of-plane buckling mode. The measured shear strength and shear modulus may be affected by the specimen dimensions and/or physical constraints. As a result, the method is referred to as a "standard guide" instead of a "standard method" by the ASTM. As was stated earlier, the tests produce a uniform state of shear stress over most of the test section/sections, however, it is accompanied by normal stresses having significant magnitude in the transverse direction. The existence of the transversal normal stress causes a failure mode that is a combination 
of shear and normal stresses. Garcia et. al. (1980) recognized the existence of the transverse normal stresses as the cause of premature failure in the $0^{\circ}$ specimens compared to the $90^{\circ}$ specimens. Sun and Yamada (1982) also reported a difference of a factor of 2 between the strength results obtained from $0^{\circ}$ and $90^{\circ}$ specimens.

Besides the above mentioned shortfalls, the difficulty involved with the installation of the test specimen in the fixtures makes the rail-shear test methods less attractive. Moreover, the results of the ASTM round-robin on this method as reported by Lockwood (1981) showed significant scatter among the average values, indicating that the method may not be a preferred test method. It is, however, informative to mention that some researchers like Sims (1973) reported good correlation between the results obtained from rail-shear tests and other methods.

\subsection{3 $10^{\circ}$ off-axis tensile test}

In this test method, a $10^{\circ}$ off-axis unidirectional specimen is loaded under tension. Like the ASTM D3039 tensile test, the specimen is tabbed at its two ends and special precautions taken to prevent bending in the test. As shown in Fig. 2.14 strains are recorded in three different directions, usually using a Delta rosette strain gage. To account for the possible out-of-plane bending, the use of two Delta rosette strain gages attached back-to-back of the specimen is recommended [Chamis and Sinclair (1977)]. Knowing the tensile load and the strains in the $x$ and two other directions at $120^{\circ}$ enables one to determine the in-plane shear stress and the strain in the principle directions of the specimen using the following equations, respectively

$$
\begin{gathered}
\tau_{12}=0.171 \sigma_{x x} \\
\gamma_{12}=-1.282 \varepsilon_{g 1}+1.879 \varepsilon_{g 2}-0.598 \varepsilon_{g^{3}}
\end{gathered}
$$

where $\varepsilon_{g 1}, \varepsilon_{g 2}$ and $\varepsilon_{g 3}$ are the strains from strain gages 1, 2 and 3 shown in Fig. 2.14. 


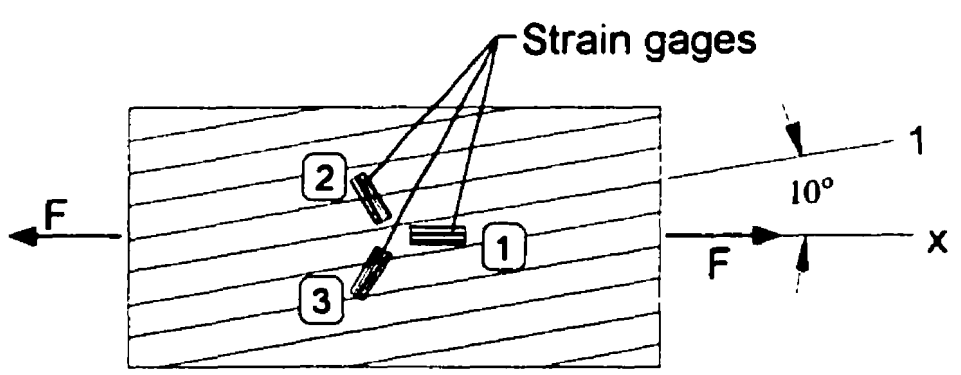

Figure $2.1410^{\circ}$ off-axis tensile test.

Chamis and Sinclair (1977) showed that when the tensile load is applied at a certain angle, depending on the property of the material, the material will experience the highest magnitude of shear stress and strain along the principal axes, while the two normal stresses $\left(\sigma_{11}\right.$ and $\left.\sigma_{22}\right)$ remain well below their critical limits. The investigation on Mod-I/epoxy, T300/epoxy and S-glass/epoxy showed that the angles were about 10, 11, and 15 degrees respectively, considered to be close enough to $10^{\circ}$. The test is very sensitive to a small misorientation error of strain-gage positioning and load alignment with respect to the fiber direction. The end constraints of the specimen in the grips can produce considerable error in the result of the test. However, the error can be reduced significantly by selecting a relatively long specimen (see for example Pindera and Herakovich (1986)). The failure of the specimen occurs under a combined state of stresses, therefore, the strength value predicted by the method is an underestimated value. On the other hand, as the analytical results of Pindera and Herakovich (1986) show, the method in general overestimates the shear modulus. These findings were also confirmed by Chiao et. al. (1977). Pindera et. al. (1987) do not recommend the method for the measurement of the shear strength. They believe that the $45^{\circ}$ off-axis tensile test provides more accurate results for shear modulus as compared to the $10^{\circ}$ off-axis test.

The $10^{\circ}$ off-axis tensile test has the advantage of using specimens with a simple geometry. However, measuring strains in three directions makes the method expensive. Moreover, as 
was stated earlier, the method is sensitive to small misalignment of the strain gages and to the direction of the load with respect to the fibers. These are in addition to the bending effect which is a common problem in all tensile tests. Nevertheless, Yeow and Brinson (1978) and Lee et. al. (1990) reported good agreement between the results obtained by the $10^{\circ}$ off-axis test and the other acceptable methods when specimens with sufficiently long length were used and/or the appropriate correction factor was applied. Lee and Munro (1986), who evaluated nine in-plane shear test methods, ranked the method below the $\pm 45^{\circ}$ and the losipescu shear methods, as the most promising testing method. They considered several parameters such as the cost of fabrication, testing cost, data reproducibility and the accuracy of the experimental results as the criteria for their ranking. It is, however, informative to mention that some researchers like Munjal (1989) do not recommend the method for the evaluation of the shear properties of FRPC.

\subsubsection{Tensile test on $\pm \mathbf{4 5}^{\circ}$ symmetric laminate (ASTM D3518-94)}

When a $\pm 45^{\circ}$ symmetric laminate is subjected to a uniaxial tensile stress in $0^{\circ}$ direction, the shear stresses along the principal axes of the laminae are independent of the material properties and equal to half of the applied tensile stress. This characteristic is the basis of the ASTM D3518 test method. The configuration of the test is shown in Fig 2.15. The specimen usually does not need tabs. Two strain gages are used to record the longitudinal and the transversal strains. Use of four strain gages, two at each face, is recommended to account for any possible bending. This enables one to calculate the shear strain along the principal direction of the laminate as follows

$$
\gamma=\varepsilon_{1}-\varepsilon_{2}
$$

where $\varepsilon_{1}$ and $\varepsilon_{2}$ are the longitudinal and the transversal strains, respectively. Simultaneous recording of the applied load and the strain enables one to determine the shear stress-strain relationship of the specimen. 


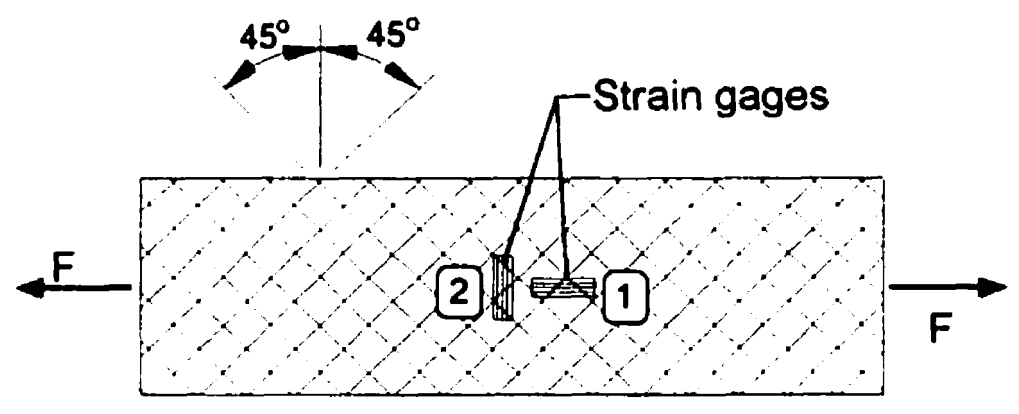

Figure $2.15 \pm 45^{\circ}$ tensile test.

Tensile test on a $\pm 45^{\circ}$ symmetric laminate for determining the in-plane properties of the FRPC was first proposed by Petit (1969). The method was later improved by Rosen (1972). This test method is believed to provide reliable information for the shear stressstrain relationship of FRPC materials well into the nonlinear region. The fact was reported by Chiao et. al. (1977), Terry (1979) and Hahn (1973). The method is also highly recommended by several researchers (see for example Munjal (1989) and Lee and Munro (1986)). In a recent investigation, Dickson et. al. (1995) employed a new method to evaluate the accuracy of the $\pm 45^{\circ}$ tensile tests along with the $10^{\circ}$ off-axis tensile and the Iosipescu shear test. In their method, they used the shear modulus obtained by these test methods to determine the tensile moduli of several shear-sensitive laminates. The comparison of the determined values with those obtained with experiments proved the $\pm 45^{\circ}$ tensile test to be the most accurate method.

Despite the wide acceptance of the $\pm 45^{\circ}$ tensile test, the shear strength evaluated by the method should be used with caution, since it does not represent the true strength value of material. A premature failure may occur due to the existence of a complex stress field close to the free edges and also due to in-plane stress normal to the fiber direction in each ply. The existence of a complex state of stresses in the vicinity of free-edges, when a laminated composite is subjected to uniaxial load, has been shown by different methods in the literature. For example, Rybicki (1971) and Conti and De Paulis (1985) presented 
approximate solutions for the determination of these stresses, while Pipes and Pagano (1970) and Isakson and Levy (1971) employed finite difference and finite element solutions, respectively. The influence of these stresses on the strength of the laminate and the possibility of the initiation of delamination failure from the free edges were discussed by Soni and Kim (1986), Herakovich (1981) and Pipes et. al. (1973). From these works, it becomes clear the failure of $\mathrm{a} \pm 45^{\circ}$ tensile specimen does not occur under pure shear.

Kellas et. al. (1993) believe that the free-edge effect is not as significant as the effect of the in-plane normal stress. They state that an in-plane stress normal to the fiber direction exists in all plies. However, the effect of this stress on a given ply is minimized by the reinforcing fibers of the neighboring plies. Since the ply constraint is reduced by the increase of the ply thickness, the thickness of each individual ply is an important parameter that influences both shear stress-strain response and the ultimate failure load of this specimen. Moreover, the surface plies of a given specimen, being constrained by only one neighboring ply, will experience higher normal stresses compared to the interior plies. Therefore, when a $\pm 45^{\circ}$ specimen undergoes tension, a combination of shear and in-plane normal stresses will initiate failure in the surface plies. The laminate can still carry more load if the remaining intact plies are capable of carrying the total applied load. It is obvious that the higher the total number of plies, the greater the chance that the remaining plies will be able to carry the load without sudden ultimate failure.

The other problem discussed by Kellas et. al. (1993) was the effect of fiber scissoring which occurs due to large deformation. This phenomenon changes the direction of the fibers from $\pm 45^{\circ}$. As a result, the accuracy of the test procedure becomes questionable.

Although the $\pm 45^{\circ}$ tensile test method provides a simple and efficient mean for evaluating the in-plane shear properties of the laminates, it requires specimens with $\pm 45^{\circ}$ lay-up 
sequence. Thus, it cannot evaluate the properties of laminates with other types of lay-up or randomly oriented chopped-fiber composites which are commonly used in practice.

\subsubsection{Iosipescu shear test (ASTM D5379-93)}

A small flat rectangular specimen having symmetrically located V-notches at its mid-length is used in this method. The configuration of the test apparatus is shown in Fig. 2.16. During the test, the specimen undergoes two counteracting moments and shear forces at the two sides of the notches. The induced moments cancel out at the mid-length of the specimen, thereby creating a state of pure shear force at the section. The $\mathrm{V}$-notches are there to promote a uniform distribution of shear stress at the section. Two strain gages (at least) are used to record the strains at $+45^{\circ}$ and $-45^{\circ}$ angle with respect to the longitudinal axis of the specimen (the $x$ axis). If the specimen is likely to twist, one must use four strain gages, two on each face. By simultaneous recording of the applied load and the strains, one can determine the shear stress-strain relationship. The following equations are used for the determination of shear stress and strain.

$$
\begin{gathered}
\tau=\frac{F}{b h} \\
\gamma=\varepsilon_{+45}-\varepsilon_{\rightarrow 5}
\end{gathered}
$$

where $h$ is the width of the specimen between the notches and $b$ is the thickness of the specimen.

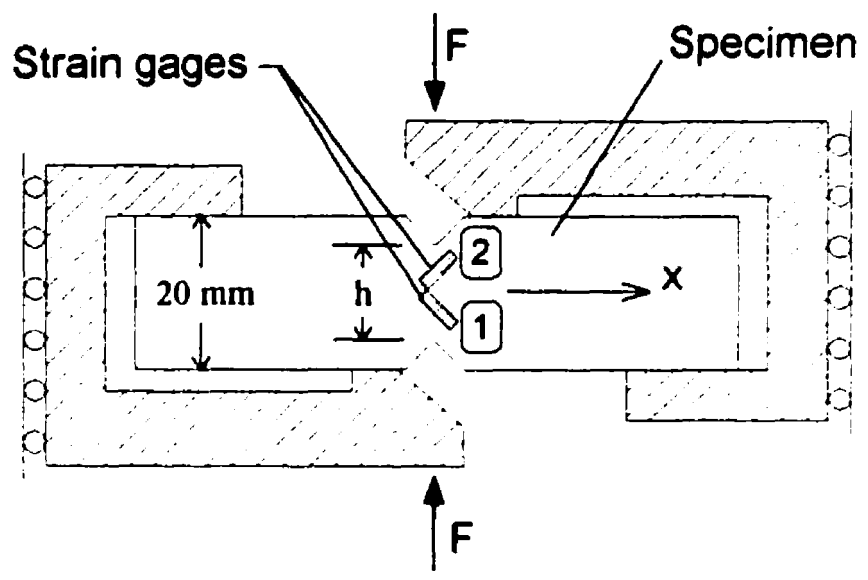

Figure 2.16 Iosipescu shear test. 
The Iosipescu shear test was originally developed for metals by Iosipescu (1967) and was later adopted for determining the shear properties of composite materials. Two alternatives of this method are the method developed by Arcan et. al. (1978) and the antisymmetric-four-point-bend method developed by Slepetz et. al. (1978). When compared with the other available methods, the Iosipescu shear test is simpler and provides reliable results. It can be used for measuring properties of unidirectional, crossply laminates and randomly oriented chopped-fiber composites. By this method, one can obtain both the in-plane ( $1-2$ direction) and the through-the-thickness (1-3 and 2-3 directions) shear properties of FRPC, depending on the direction of the specimen in the fixture. However, for the through-the-thickness shear properties, one needs specimens with $20 \mathrm{~mm}$ thickness, which are usually difficult to prepare. Lee and Munro (1986) ranked the losipescu shear test beside the $\pm 45^{\circ}$ tensile test as the most practical technique currently available for testing FRPC. Walrath and Adams (1983) and Adams and Walrath (1987) also considered the method as one of the most versatile methods for measuring the shear properties of FRPC.

Many experimental investigations have been carried out in which the results obtained by the Iosipescu method were compared with the results of the other methods. For example Sawnson et. al. (1985) compared the results of the losipescu test with those of the torsion tube test. Lee et. al. (1990) compared the results of the Iosipescu test with those of the $\pm 45^{\circ}$ and the $10^{\circ}$ off-axis tensile test. Pierron et. al. (1995) used an isotropic material and determined the shear properties of the material indirectly from the tensile test for the comparison with the results of losipescu test. The results of these investigations confirmed the Iosipescu to be an acceptable test method.

Despite the wide acceptance of the Iosipescu shear test, the result obtained from this method should not be considered as the true shear properties of the material. This is due 
to the fact that a uniform and pure shear stress state does not exist in the gage section of the specimen. Investigations by Pinder et. al. (1987) and Morton et. al. (1992) showed that the method overestimated the shear modulus of the material when $0^{\circ}$ specimens were examined, while the evaluated values from $90^{\circ}$ specimens were always underestimated. Pinder et. al. (1987) showed that the margin of the discrepancy depending on the degree of the anisotropy of the material might reach $40 \%$. Abdallah and Gascoigne (1989) reported that the best results for shear modulus could be obtained from $\left[0^{\circ} / 90^{\circ}\right]$ and $\left[0^{\circ} \pm 45^{\circ} / 90^{\circ}\right]$ specimens. This fact was also reflected in ASTM D5379-93.

Since a state of pure shear stress does not exist in the gage section, the specimen usually fails under a mixed-failure mode. This problem, beside the nonuniformity of the shear stress in the gage section, promotes a premature failure of the specimen. The finite element analyses conducted by Herakovich and Bergner (1980) and Abdallah et. al. (1989) showed the severity of the problem in $0^{\circ}$ specimens. Therefore, they suggested $90^{\circ}$ specimens be used for the determination of shear strength. Sullivan et. al. (1984) conducted similar work, however, they supported their findings by photoelasticity on vinyl-ester resin. Their numerical and photoelastic results indicated that for isotropic material better results could be obtained by the antisymmetric four-point-bend method compared to the common Iospescu fixture known as Adams and Walrath fixture.

Gipple and Hoyns (1994) conducted a comprehensive investigation on the Iosipescu shear test when used for the through-the-thickness shear properties of FRPC. They measured the shear strain at the gage section of the specimens by using conventional strain gages, full section strain gages and moire interferometry technique. They supported their results by non-linear finite element analyses. Their findings confirmed the findings of the previous researchers. 
The influence of the load location on the specimen and the notch geometry on the test result was investigated by Spigel et. al. (1987). They reported that these parameters significantly influenced the test results. Measurement of strains also has significant effect on the test result. That is because the area of pure shear is very small. Abdallah and Gascoigue (1989) reported that this area was about $1.5 \mathrm{~mm}$ long, and therefore they suggested strain gages with a gage length of $1 \mathrm{~mm}$ be used for the test.

\subsection{Through-the-thickness shear tests}

Evaluation of the through-the-thickness shear properties of FRPC is more involved than their in-plane properties. Among the various methods discussed in the above, only the Iosipescu method can be used for the evaluation of through-the-thickness shear properties. However, the method requires specimens with $20 \mathrm{~mm}$ thickness, which are not easy to prepare. Because of this difficulty, the method is not popular in the industry, and the related experimental works in the literature are very limited (see for example Gipple et. al. (1994)). The other shear test methods that enable one to produce a through-the-thickness shear stress in the materials are the short-beam shear test (ASTM D2344-84) and the notched-specimen test (ASTM D3846-94). These methods are discussed in below.

\subsubsection{Short-beam shear test (ASTM D2344-84)}

When a FRPC specimen is subjected to three-point bending test, failure will occur due to excessive bending moment, while the shear force will remain far below the specimen shear strength. This phenomenon is reversed by selecting a specimen with small span-to-depth ratio (usually $L / h \leq 5$ ) in the short-beam shear test. Assuming a parabolic shear distribution through the thickness of the beam, the shear strength is calculated from the following equation

$$
\tau_{\max }=\frac{3 F_{\max }}{4 b h}
$$


where $F_{\max }$ is the failure load and $b$ and $h$ are the width and the depth of the beam, respectively. No practical method is available for determining the shear modulus from this method.

In an early investigation, Sattar and Kellogg (1969) concluded that the failure in the shortbeam shear test was a pure shear failure starting from the center line of the beam. They reasoned that the combination of shear and flexure at intermediate locations was not of sufficient magnitude to cause failure. They had established their conclusions on the assumption of a parabolic distribution of shear stress through the thickness of the beam, which was later proved to be incorrect by other researchers. For example, Berg et. al. (1972) used the finite element method to determine the correct distribution of the stresses in the short-beam shear test while Whitney and Browning (1985) found the elasticity solution of the problem. Both investigations showed that the distribution of shear stress in the sections along the specimen is not even close to parabolic in shape. Indeed, the maximum shear stress occurs close to the top of the beam adjacent to the loading nose where high magnitude longitudinal compression stresses exist. The combination of shear and compression stresses at this region produces a mixed failure mode which cannot be correlated to the shear strength calculated from Eqn (2.12). Xie and Adams (1995) and Chatterjee (1996) also confirmed these findings. As a result the value obtained from Eqn (2.12) is referred to as the "apparent shear strength" by ASTM D2344-84 and the method is only recommended for screening and quality control.

\subsubsection{Notched-specimen test method (ASTM D3846-94)}

The shear strength of the material is measured by applying a compressive load to a notched specimen with the configuration shown in Fig. 2.17. The specimen is located in a jig similar to that of the ASTM D695 test method (Fig. 2.6) so that it cannot buckle laterally. Assuming a shear failure along line $a b$, the shear strength is the failure load divided by the shear area between the two notches. 


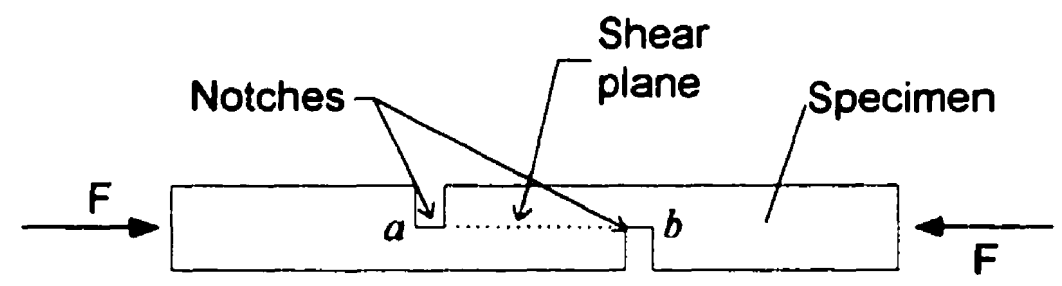

Figure 2.17 Notched-specimen test method.

The notched-specimen test does not provide the true shear strength of the material, since the shear stress is not pure and uniform over the specified gage length. The work conducted by Shokrieh (1995) indicated the existence of high stress concentration at notch regions. Consequently, a mix-failure initiating at the tip of the notches governs the strength of the specimen. As a result, as Chiao et. al. (1977) reported, the method underestimates the shear strength of the materials. Since the degree of stress concentration depends on the geometry of the notches, the results of the test is quite sensitive to the notch machining. This method was originally designed to accommodate those types of FRPC that could not be adequately characterized by the short-beam test. However, because of the difficulty involved in the precise machining of the notches and the above mentioned shortfalls, the method has not gained popularity. Munjal (1989) did not recommend the method even for quality control.

\subsection{Recent achievements}

The anisotropic nature of composite materials and the difficulty involved with the measurement of their elastic properties have always required the development of new test methods and the modification of available ones. However, each new technique must be validated through several investigations before it could be considered an acceptable testing method. Even after this stage, the method may not gain popularity, since the popularity of a given method depends mainly on the practicality of the test and the associated data processing effort. A few recent achievements in this field are reviewed below. 
Tsai and Danial (1990) and Tsai et. al. (1990) developed a new test method in which thin rectangular coupons were subjected to torsion. A closed form solution was obtained based on the Mindlin-Reissner theory [Mindlin (1951)]. In this method, the strains on the width and the thickness of the specimen must be measured at $45^{\circ}$ angle with respect to longitudinal axis. With a $0^{\circ}$ specimen one can obtain both $G_{12}$ and $G_{13}$ of the material. However, at least two tests on specimens having different width-to-thickness ratios are needed. The data from the tests, then, are used in a trail and error procedure to determine the two shear moduli simultaneously. To obtain $G_{23}$, one must use a $90^{\circ}$ specimen. When compared with the tube torsion test, the new method uses specimens with simple geometry. However, it requires a relatively complicated trail and error procedure to extract the shear moduli from the test data. Moreover, the accuracy of the MindlinReissner theory for this problem needs further investigation.

Short (1995) proposed a new test method that enabled one to obtain the through-thethickness shear strength of composite materials. In his method a sandwich specimen composed of two steel facings and composite core is subjected to four point bending. The span-to-depth ratio of the specimen is selected such that failure occurs due to interlaminar shear inside the core material. Short (1995) used classical laminated plate theory (CPLT) to determine the maximum shear stress at the center line of the specimen. He reasoned that the existence of the steel plates at two sides of the composite core prevented the stress concentration in the vicinity of the loads and reactions, therefore, the method did not have the problems associated with the short-beam shear test. Nevertheless, the reported shear strength values obtained by this method and short-beam shear test did not show significant differences. Furthermore, the validity of the existence of pure shear stress at the centerline of the specimen and the adequacy of CLPT in this method need to be investigated by finite element analysis or by some other numerical/mathematical methods. 
Bansal and Kumosa (1995) and Broughton et. al. (1990) developed a new fixture that enabled them to subject the Iosipescu shear test specimen to a combined shear and axial stress. Their fixture is good for characterization of mixed-failure modes of the material, however it is not a suitable device for common use in industry and research centers.

\subsection{Summary and conclusions}

Several test methods available for characterizing the mechanical properties of FRPC were discussed in this chapter. Among them, some are only suitable for quality control and screening, and some are too costly and time consuming or require complicated data processing. As a result, there remain only a few popular test methods that can provide acceptable data for design purposes. These methods are the strip-specimen tension test (ASTM D3039-93), the side-loaded specimen compression test (ASTM D3410-95), the $\pm 45^{\circ}$ tensile test (ASTM D3518-94) and the Iosipescu shear test (ASTM D5379-93). However, each of them, as was discussed in detail, has some shortfalls. Consequently, several attempts have been made to develop new test methods or modify available test methods to overcome the existing shortfalls. One may consider the ideal test method as a method that has the following attributes.

a) subjects the specimen to a state of stress that is similar to the state of stress that the material experiences in its actual life.

b) can use as-received specimens (e.g. does not require a specimen with special lay-up sequences).

c) uses specimens with simple geometry.

d) does not require time consuming alteration and machining of the specimens.

e) does not require considerable effort for mounting the specimen in the fixture.

f) does not require strain gages and/or other expensive instruments.

g) produces reliable results.

h) can provide several properties from the same set of tests. 
Although in most engineering applications FRPC are subjected to a combined state of stresses, the general trend in the design of testing methods has focused on producing a state of pure stress along a gage length within a given specimen. As a result, the evaluated properties from these methods may not represent the true in service behavior of the materials. On the other hand, when there are test methods that subject the materials to a combined state of stress, the interpretation of the test data either is not easy or the data are not useful for design purposes. Furthermore, the state of stress produced by the test method may not be close to the one that the material may experience in its service life. Item $a$ in the above list addresses this problem.

The development of the Varying-Span Method (VSM) is a step towards the development of an ideal test method. The method is an extension of the three-point bending test and, therefore, it has all the advantages of this method. On the other hand, the proposed modification to the three-point bending test eliminates its associated shortfalls and consequently makes the VSM a relatively ideal test method. With this method, specimens with different span-to-depth ratios $(L / h)$ are subjected to three-point bending from which one can determine the longitudinal modulus and the through-the-thickness shear modulus of the material. The flexural strength and the apparent interlaminar shear strength can also be obtained from the results of the same tests, however the method does not lend anything new to the evaluation of these two properties. Therefore, they will not be addressed in this thesis.

Also among the several advantages of the VSM is its capability of evaluating the throughthe-thickness shear modulus of the FRPC from thin specimens (say $2 \mathrm{~mm}$ thick). The evaluation of this property, as was explained when reviewing the different test methods in this chapter, is not a simple task. For instance, the Iosipescu shear test, which is capable of providing this property, requires $20 \mathrm{~mm}$ thick specimens; a thickness which is not easily 
achieved. On the other hand, the torsion test method developed by Tsai and Danial (1990) and Tsai et. al. (1990) has the shortfalls that a) it is involves complicated data interpretation and b) it requires a torsion machine which may not be available in many facilities. Therefore, besides other positive attributes, the VSM is expected to receive special credit for its ability to evaluate the through-the-thickness shear modulus. 


\section{Chapter 3}

\section{THE EXACT SOLUTION FOR SIMPLY SUPPORTED BEAMS UNDER THREE-POINT BENDING}

Although the analysis of a simply supported beam is a simple task in structural mechanics, its exact solution is lengthy and complicated. The Euler beam theory is adopted in structural mechanics. This theory produces fairly accurate results for beams with large span-to-depth ratios $(L / h)$, however, it is unable to accurately predict the behavior of the beams with small $L / h$. Moreover, as was shown in Fig. 2.12 , the validity of the theory in proximity of the concentrated loads is questionable. The exact solution for such a beam can be obtained by the application of theory of elasticity. Sandorff (1980) used the finite difference method to solve the differential equation of the theory of elasticity for the threepoint bending boundary conditions. Berg et. al. (1972) and Xie and Adams (1994) used the finite element method to analyze the problem. Two different closed form solutions proposed by Whitney and Browning (1985) and Chatterjee (1996) are also available in the literature.

Chatterjee (1996) used a Fourier transform solution for the elasticity problem of an infinite orthotropic strip. Although the solution is for an infinite strip, it gives accurate results for beams of finite length when the overhang lengths are about two times the depth of the beam. In this solution, the loads must be divided into antisymmetric and symmetric loads. The final result is obtained by superposing the results of the analyses of the antisymmetric and the symmetric loads. A numerical integration is employed for the Fourier transformations.

Whitney and Browning (1985) and Whitney (1985) solved the problem for a beam of finite length. The solutions were given for three- and four-point bending problems with the assumption that the concentrated loads and the reactions are distributed uniformly over 
small lengths. In their solution, the shear stress at the free ends of the beam automatically vanishes by the nature of the employed stress function. However, the boundary condition of $\sigma_{\mathrm{xx}}=0$ at the free ends were not be fully satisfied. Instead, the force and moment resultants are set to zero at the two ends. Although this brings approximation into the solution, the solution for beams with sufficient overhangs, which is usually the case for laboratory specimens, practically leads to zero longitudinal stresses at the two ends. As a result, from a practical standpoint, the solution satisfies the required boundary conditions.

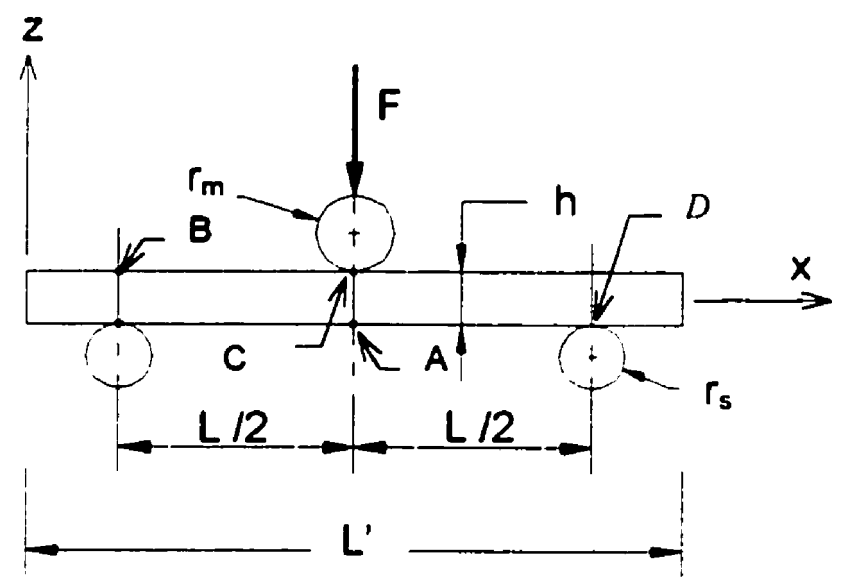

Figure 3.1 Geometry of simply supported beam for analytical solution.

In this chapter we solve the problem of the simply supported orthotropic beam with a new approach. The configuration of the problem is shown in Fig. 3.1. The distribution of the concentrated load and the reactions are assumed to follow the Hertizian contact law. The solution satisfies the conditions of zero longitudinal stress $\left(\sigma_{x x}=0\right)$ and zero shear force at the two free ends of the beam $\left(x=0, L^{\prime}\right)$. In general, the solution is simpler and more representative of the problem of three-point bending as compared to the two existing closed form solutions. The solution will be used for the evaluation of VSM and the through-the-thickness inextensibility theory later. First we will find the solution of a simply supported beam subjected to distributed harmonic loads on both sides. The solution will then be extended to the beam subjected to three-point bending. We will define a new 
terminology called the "net mid-span deflection". Subsequently, the effects of variables such as the transverse modulus of elasticity $\left(E_{33}\right)$, the Poisson ratio, the diameter of the loading nose and the magnitude of the load on the net mid-span deflection are investigated.

\subsection{Solution for harmonic loading}

Consider the orthotropic beam shown in Fig. 3.2. To produce such a beam from FRPC, the fibers must be either in the $x$ direction or perpendicular to the $x z$ plane. The boundary conditions at two ends are defined as follows

$$
\begin{gathered}
\sigma_{x x}(0, z)=\sigma_{x x}\left(L^{\prime}, z\right)=0 \\
w(0, z)=w\left(L^{\prime}, z\right)=0
\end{gathered}
$$

where $w$ is the dispiacement in the $z$ direction. As shown in the figure, sinusoidal loads with different amplitudes, but with the same wave length act on the top and the bottom surfaces of the beam. These loads are defined by the following functions

$$
\begin{aligned}
& q_{t}(x)=a_{n}{ }^{\prime} \sin (p x) \\
& q_{b}(x)=a_{n}{ }^{b} \sin (p x)
\end{aligned}
$$

where $a_{n}^{t}$ and $a_{n}^{b}$ are the amplitudes of the top and the bottom loads, respectively, and

$$
p=\frac{n \pi}{L^{\prime}} \quad n=1,2,3, \ldots
$$

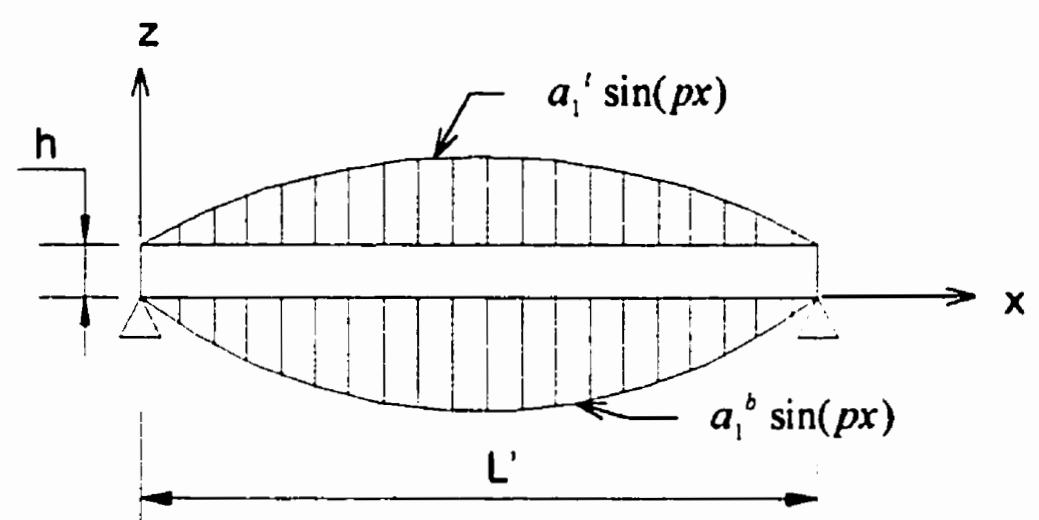

Figure 3.2 Simply supported beam subjected to harmonic loading on both its surfaces 
Idealizing the orthotropic beam as a plane stress problem, the following equations define the stress-strain relationships within the beam

$$
\begin{aligned}
& \varepsilon_{x x}=\bar{S}_{11} \sigma_{x x}+\bar{S}_{13} \sigma_{x:} \\
& \varepsilon_{:=}=\bar{S}_{13} \sigma_{x x}+\bar{S}_{33} \sigma_{:=} \\
& \gamma_{x=}=\bar{S}_{66} \tau_{x}
\end{aligned}
$$

where $S_{\eta}$ are the compliance coefficients of the material. These coefficients are defined by

$$
\begin{aligned}
& \bar{S}_{11}=\frac{1}{E_{x x}} \\
& \bar{S}_{33}=\frac{1}{E_{x z}} \\
& \bar{S}_{13}=-\frac{\mathrm{u}_{x e}}{E_{x z}} \\
& \bar{S}_{66}=\frac{1}{G_{x e}}
\end{aligned}
$$

where $E, G$ and $v$ are the elastic modulus, shear modulus and the Poisson's ratio of the material, respectively. Notice that as the definition of the orthototropic beam implies, the principal axes of the material must be along the axes of the $x y z$ coordinate system of the beam. Otherwise, Eqn (3.4) will not be valid. The equations of equilibrium for the state of plane stress are as follows

$$
\begin{aligned}
& \frac{\partial \sigma_{x x}}{\partial x}+\frac{\partial \tau_{x z}}{\partial z}=0 \\
& \frac{\partial \sigma_{z=}}{\partial z}+\frac{\partial \tau_{x}}{\partial x}=0
\end{aligned}
$$

The solution of differential equations (3.6) satisfying Eqns (3.1) through (3.4) was given by Pagano (1969) in the following form

$$
\begin{aligned}
& \sigma_{x x}=\sin (p x) \sum_{i=1}^{4} A_{i} m_{i}^{2} \exp \left(m_{t} z\right) \\
& \sigma_{z=}=-p^{2} \sin (p x) \sum_{i=1}^{4} A_{i} \exp \left(m_{i} z\right) \\
& \tau_{x=}=-p \cos (p x) \sum_{i=1}^{+} A_{i} m_{i} \exp \left(m_{i} z\right)
\end{aligned}
$$


and

$$
\begin{aligned}
& u=\frac{\cos (p x)}{p} \sum_{i=1}^{4} A_{i}\left(\bar{S}_{13} p^{2}-\bar{S}_{11} m_{1}^{2}\right) \exp \left(m_{1} z\right) \\
& w=\sin (p x) \sum_{i=1}^{4} A_{t}\left(\bar{S}_{13} m_{1}-\frac{\bar{S}_{33}}{m_{1}} p^{2}\right) \exp \left(m_{1} z\right)
\end{aligned}
$$

where $u$ and $w$ are the displacement components in the $x$ and $z$ directions, respectively. The four values of $m_{i}$ are defined by the different combinations of the following equation.

$$
m_{1}= \pm p \sqrt{\frac{a \pm d}{e}}
$$

where

$$
\begin{aligned}
& a=\bar{S}_{66}+2 \bar{S}_{13} \\
& d=\sqrt{a^{2}-4 \bar{S}_{11} \bar{S}_{33}} \\
& e=2 \bar{S}_{11}
\end{aligned}
$$

$A_{1}$ (for $i=1$ to 4 ) are unknown constants which will be determined by satisfying the boundary conditions on the top and the bottom surfaces of the beam. These boundary conditions are

$$
\begin{gathered}
\tau_{x=}(h / 2)=\tau_{x=}(-h / 2)=0 \\
\sigma_{z=}(h / 2)=q_{1}(x) \\
\sigma_{z=}(-h / 2)=q_{b}(x)
\end{gathered}
$$

Equations (3.11) and (3.12) in conjunction with Eqn (3.7) provide four equations so that one can solve them for the four unknowns. Knowing $A$, the components of displacement and stress can be easily determined from Eqns (3.7) and (3.8).

It is important to note that the solution provided is only for one term of the Fourier series. However, since any load can be transformed into a Fourier series, the above solution can be used for general purposes. For this, the behavior of the beam under each term of the series is determined separately. The final result will, then, be obtained by superposition of 
the results associated with each term of the Fourier series. This procedure will be used for the solution of a beam under three-point bending next.

\subsection{Solution for beam under three-point loading}

Consider the beam shown in Fig. 3.3. This beam is to represent the simply supported beam shown in Fig. 3.1, The beam has a total length of $L^{\prime}$ while the span between the two supports is $L$. The mid-span load and the reaction forces are distributed over small lengths representing the contact phenomenon. As shown in the figure, there are two fictitious supports at the two ends of the beam so that one can use the solution provided for the harmonic loads in section 3.1. Since the loads at the top and the bottom of the beam are in equilibrium, the supports do not apply any forces to the beam. As a result, the solution of the beam in Fig. 3.3 provides the correct answers, except that it includes a rigid body movement equal to the vertical displacement of the points located at the real supports $\left(w_{D}\right)$. As a result, to determine the absolute values of the vertical displacements, one must deduct the rigid body displacement from the displacement values obtained by the solution.

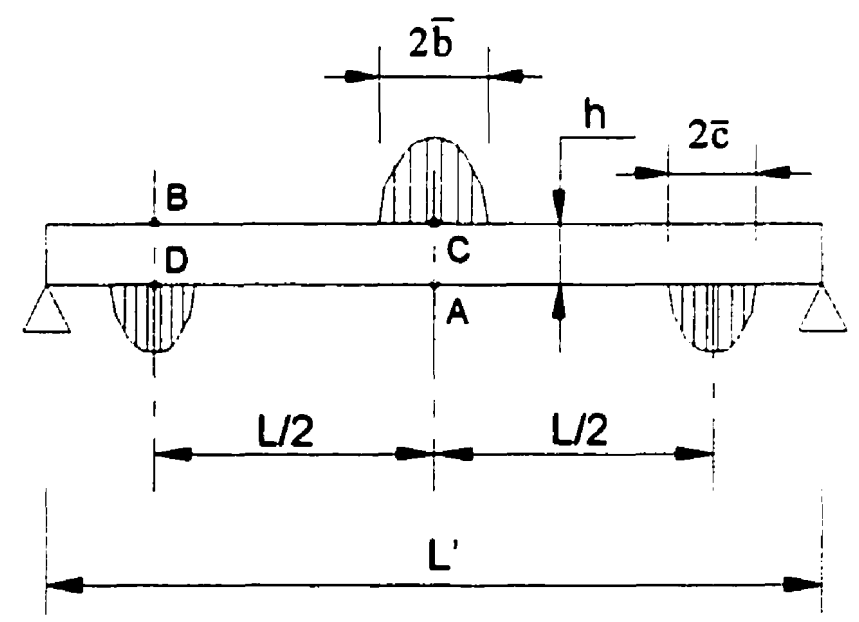

Figure 3.3 Idealization of a beam subjected to three-point bending.

The solution of the beam shown in Fig. 3.3 satisfies the boundary condition of zero longitudinal stress $\left(\sigma_{x x}=0\right)$ at the two free ends of the beam. However, it does not 
guarantee the condition of zero shear stress at beam's two ends. Instead, the condition of zero shear force, expressed by the following equation, exists.

$$
\int_{-h / 2}^{h / 2} \tau_{x z} d z=0 \text { at } x=0, L^{\prime}
$$

Because of the effect of the local reaction forces, the distribution of the shear stress in the vicinity of the supports is irregular. As a result, despite the fact that there is no shear force in the sections located between the supports and the free edges, shear stresses with considerable magnitude may exist. However, as was shown by Jalali and Taheri (1998a), the irregularity of the shear stress practically vanishes when the section has a distance equal or greater than the beam thickness $(h)$ from the point of application of local force. Therefore, for beams with overhangs larger than the beam thickness (a situation that always exists in laboratory specimens), Eqn (3.13) practically results in the condition of zero shear stress at the two free ends.

It is also instructive to mention that the solution implies the restriction of equal vertical displacement for the points located on the two free end sections. Although this restriction is against the definition of free ends, such a situation practically exists in beams with overhangs equal or bigger than the beam thickness. This is due to the fact that sections located far enough from the supports (Fig. 3.1) are free from stresses, and as a result no relative vertical movement may occur in those sections. In summary, since the overhangs of the laboratory specimens are bigger than the minimum amount required, the solution of the beam in Fig. 3.3 accurately represents the true behavior of the acual test specimens.

As was stated earlier and as shown in Fig. 3.3, the concentrated load at mid-span and the reactions are distributed over finite widths. This phenomenon is due to the deformation of the contacting bodies under the applied load. A comprehensive discussion on this subject was presented by Goldsmith (1960) and Timoshenko and Goodier (1970). They applied the Hertzian contact law to determine the distribution of the stresses and the area of 
contacts for isotropic elastic materials. Sankar (1989) included the effect of bending in his solution and Chatterjee (1996) presented a solution for anisotropic materials. However, none of these solutions has the simplicity of the solution based on the Hertzian contact law. Since the use of different contact laws does not alter the outcome of our investigation, we adopt the Hertzian contact law which has the advantage of simplicity. According to this, the width of contact for the mid-span concentrated load can be defined by

$$
2 \bar{b}=4 \sqrt{F\left(\delta_{1}+\delta_{2}\right) r_{m}}
$$

where $\bar{b}$ denotes half of the contact width and $r_{m}$ is the radius of the loading nose. Also

$$
\begin{aligned}
& \delta_{1}=\frac{1}{E_{1} \pi} \\
& \delta_{2}=\frac{1}{E_{2} \pi}
\end{aligned}
$$

where $E_{1}$, and $E_{2}$ are the elastic moduli of the two contacting bodies. The distribution of load over the contact width is slightly modified from the one defined by the Hertizian contact law to simplify the mathematical manipulations. For the mid-span load, the distribution is defined by the following equation

$$
q=\frac{\pi F}{4 \bar{b}} \cos \left(\frac{\pi \bar{x}}{2 \bar{b}}\right)
$$

where $\bar{x}$ is measured from the center of the contact width. The contact width and the distribution of load at the supports are defined by

$$
\begin{aligned}
& 2 \bar{c}=4 \sqrt{0.5 F\left(\delta_{1}+\delta_{2}\right) r_{s}} \\
& q=\frac{\pi F}{8 \bar{c}} \cos \left(\frac{\pi \bar{x}}{2 \bar{c}}\right)
\end{aligned}
$$

where $r_{s}$ is the radius of the support roller. To solve the problem, the load on the top and the bottom of the beam must be transformed into Fourier series separately. Since the distribution of the loads with respect to the mid-span is symmetric, the Fourier series will not include even components. Therefore, the transformation of loads into Fourier series will have the general format of 


$$
q=\sum_{n=1}^{\infty} a_{n} \sin (p x) \quad n=1,3,5, \ldots
$$

where $p$ has the same definition given in Eqn (3.3). To find the coefficients of the series we multiply both sides of Eqn (3.18) by $\sin \frac{m \pi x}{L^{\prime}}$ and integrate them over the entire length of the beam. Since for $n \neq m$ the result of $\int_{0}^{L^{\prime}} \sin \frac{n \pi x}{L^{\prime}} \sin \frac{m \pi x}{L^{\prime}} d x$ is zero, the following equation is obtained for the coefficients of the Fourier series.

$$
a_{n}=\frac{2}{L^{\prime}} \int_{0}^{L^{\prime}} q \sin \frac{n \pi x}{L^{\prime}} d x
$$

By changing the variable of $x$ to

$$
x=\frac{L^{\prime}}{2}+\bar{y}
$$

and with some manipulation the result will be

$$
a_{n}=(-1)^{\frac{n-1}{2}} \frac{4}{L^{\prime}} \int_{0}^{L^{\prime} / 2} q \cos \frac{n \pi \bar{y}}{L^{\prime}} d \bar{y} \quad n=1,3,5, \ldots
$$

where, $\bar{y}$ is measured from the mid-span. Note that because of symmetry, the integration in Eqn (3.21) is only over half of the length of the beam, however a coefficient of two was applied to provide the result for the whole length. The coefficient of Fourier series for the load on the top and the load on the bottom of the beam must be calculated separately. To determine $a_{n}$ for the load applied on the top surface of the beam, we substitute Eqn(3.16) into Eqn (3.21). By replacing $\bar{y}$ with $\bar{x}$ we obtain

$$
a_{n}{ }^{\prime}=(-1)^{\frac{n-1}{2}} \frac{\pi F}{\bar{b} L^{\prime}} \int_{0}^{\bar{b}} \cos \frac{\pi \bar{x}}{2 \bar{b}} \cos \frac{n \pi \bar{x}}{L^{\prime}} d \bar{x} \quad n=1,3,5, \ldots
$$

This equation can be simplified to

$$
a_{n}^{\prime}=(-1)^{\frac{n-1}{2}} \frac{2 F}{L^{\prime}} \frac{\cos (p \bar{b})}{1-4\left(\frac{p \bar{b}}{\pi}\right)^{2}} \quad \text { for } \quad \frac{p \bar{b}}{\pi} \neq \frac{1}{2}
$$




$$
a_{n}{ }^{\prime}=(-1)^{\frac{n-1}{2}} \frac{\pi F}{2 L^{\prime}} \quad \text { for } \frac{p \bar{b}}{\pi}=\frac{1}{2}
$$

To obtain $a_{n}$ for the load applied to the bottom surface of the beam, the second of Eqn (3.17) is substituted into Eqn (3.21). By changing the variable

$$
\bar{y}=\frac{L}{2}+\bar{x}
$$

and with some manipulation one obtains

$$
a_{n}^{b}=(-1)^{\frac{n-1}{2}} \frac{\pi F}{2 \bar{c} L^{\prime}} \int_{-\bar{c}}^{\bar{c}} \cos \frac{\pi \bar{x}}{2 \bar{c}} \cos \left(\frac{n \pi \bar{x}}{L^{\prime}}+\frac{n \pi L}{2 L^{\prime}}\right) d \bar{x} \quad n=1,3,5, \ldots
$$

After simplification, this equation becomes

$$
a_{n}^{b}=(-1)^{\frac{n-1}{2}} \frac{2 F}{L^{\prime}} \frac{\cos (p \bar{c})}{1-4\left(\frac{p \bar{c}}{\pi}\right)^{2}} \cos \left(\frac{p L}{2}\right) \quad \text { for } \frac{p \bar{c}}{\pi} \neq \frac{1}{2}
$$

and

$$
a_{n}^{b}=(-1)^{\frac{n-1}{2}} \frac{\pi F}{2 L^{\prime}} \cos \left(\frac{p L}{2}\right) \text { for } \frac{p \bar{c}}{\pi}=\frac{1}{2}
$$

Knowing the coefficients of the Fourier series components for the mid-span concentrated load and the reactions, the stresses and displacement at each point are obtained by the superposition of the results obtained from Eqns (3.7) and (3.8) for each components. As was mentioned earlier, the $z$ direction displacement components includes a rigid body displacement equal to $w_{D}$. To determine the absolute displacement values, one should deduct the rigid body displacement from the value obtained directly from the solution. However, since the VSM utilizes relative displacements of certain points along the beam, deduction is an unnecessary operation.

\subsection{Numerical investigations}

In this section the influence of various parameters on the response of FRPC beam under three-point bending is investigated. Since the effect of the local deformations is significant for beam with small $L / h$, a beam with $L / h=4$ is considered. The geometry of this beam is 
shown in Fig. 3.4. The radii of the loading nose and the supporting rollers are assumed to be equal. This radius is considered as a variable in the investigation. The magnitude of the applied load, the modulus of elasticity of the beam in the $z$ direction and the Poisson's ratio are also considered as variables. The material properties of the beam are taken as follows

$$
\begin{aligned}
& E_{x x}=200 \mathrm{GPa} \\
& E_{z=}=8-32 \mathrm{GPa} \\
& G_{x=}=4 \mathrm{GPa} \\
& U_{x=}=0.15-0.45
\end{aligned}
$$

The properties represent materials with very high anisotropy. As a result, the local effect of the concentrated load and reaction forces is pronounced. The loading nose and the rollers are assumed to be steel with modulus elasticity of $E=200 \mathrm{GPa}$. The elastic modulus of the beam in the $z$ direction $\left(E_{z:}\right)$ is also used for the determination of contact behavior. The following quantities are defined for the purpose of the analysis.

$$
\begin{array}{ll}
\text { Net mid-span deflection: } & \Delta_{N}=w_{A}-w_{B} \\
\text { Semi-net mid-pan deflection: } & \Delta_{S N}=w_{A}-w_{D} \\
\text { Total mid-span deflection: } & \Delta_{T}=w_{C}-w_{D} \\
\text { Local deformation: } & \Delta_{L}=\Delta_{T}-\Delta_{N}
\end{array}
$$

The solution provided in this chapter was implemented into a computer program (ELAS3P) for determining the above mentioned quantities. The program was written in Qbasic and is listed in Appendix A. In this investigation, the effect of the variables on the quantities defined in Eqn (3.30) is studied. The results of the investigation are shown in Fig. 3.5 through Fig. 3.8. The results are presented in the form of either the flexural stiffness $(F / \Delta)$ or the stiffness ratio versus the variable. When the quantity $F / \Delta$ is used, $\Delta$ is the displacement according to the definitions of Eqn (3.30). The stiffness ratios are the values of $F / \Delta$ normalized with respect to the $F / \Delta$ value of a situation which will be defined for each particular case separately. 


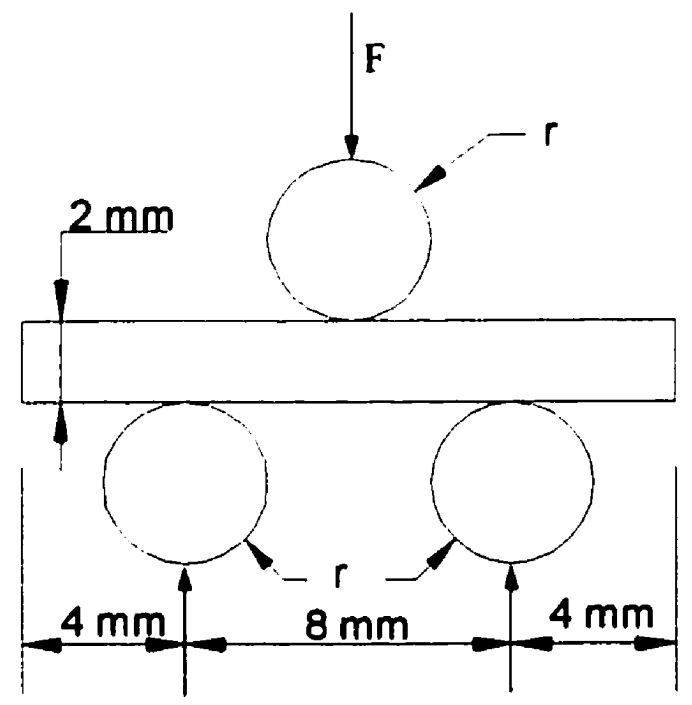

Figure 3.4 Geometry of the beam used for the numerical investigation.

Figure $3.5 \mathrm{a}$ shows the influence of load magnitude on the mid-span flexural stiffness values. As the figure shows, the behavior is nonlinear. However, the amount of nonlinearity for net mid-span flexural stiffness is insignificant. The nonlinear behavior of the flexural stiffness values is due to the increase of contact areas between the beam and the load and the support rollers as the applied load increases. The lowest stiffness value for each case is the stiffness at the start of the loading (initial stiffness). This value increases as the load increases. In Fig. 3.5b, the stiffness values are normalized with respect to the initial stiffness value. As this figure shows, the variation of the stiffness values are about $1.5 \%, 15 \%$ and $39 \%$ for the net, semi-net and total mid-span deflections, respectively. This indicates that the variation of the load does not have significant effect on the linear behavior of the net mid-span deflection.

The influence of radius of the rollers on the response of the beam is shown in Figs 3.6a and $3.6 \mathrm{~b}$. The values in Fig. 3.6b are normalized with respect to the stiffness value corresponding to $r=5 \mathrm{~mm}$. The figures show that the radius of the rollers has insignificant effect on the net mid-span deflection, while the semi-net and the total deflections are quite sensitive to this parameter. 

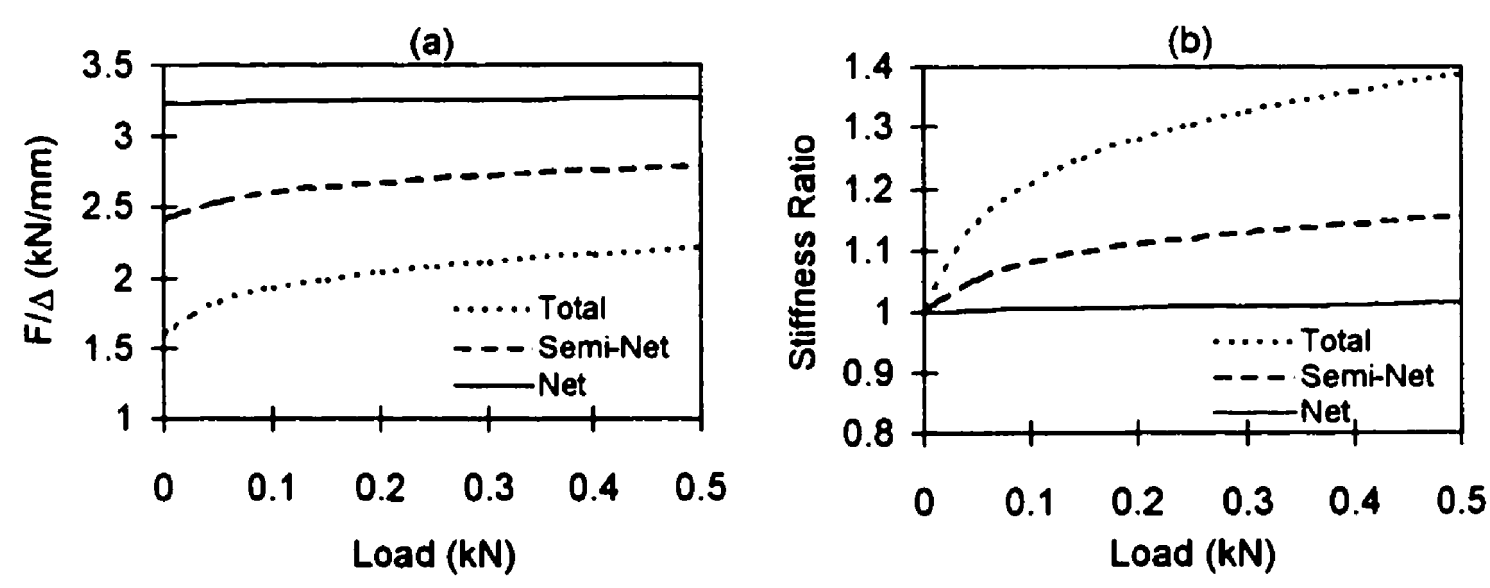

Figure 3.5 The effect of load magnitude on mid-span deflections for $r=5 \mathrm{~mm}, E_{z=}=10$ $\mathrm{GPa}$ and $v_{x e}=0.25$. (a) Non-normalized results. (b) Normalized results.
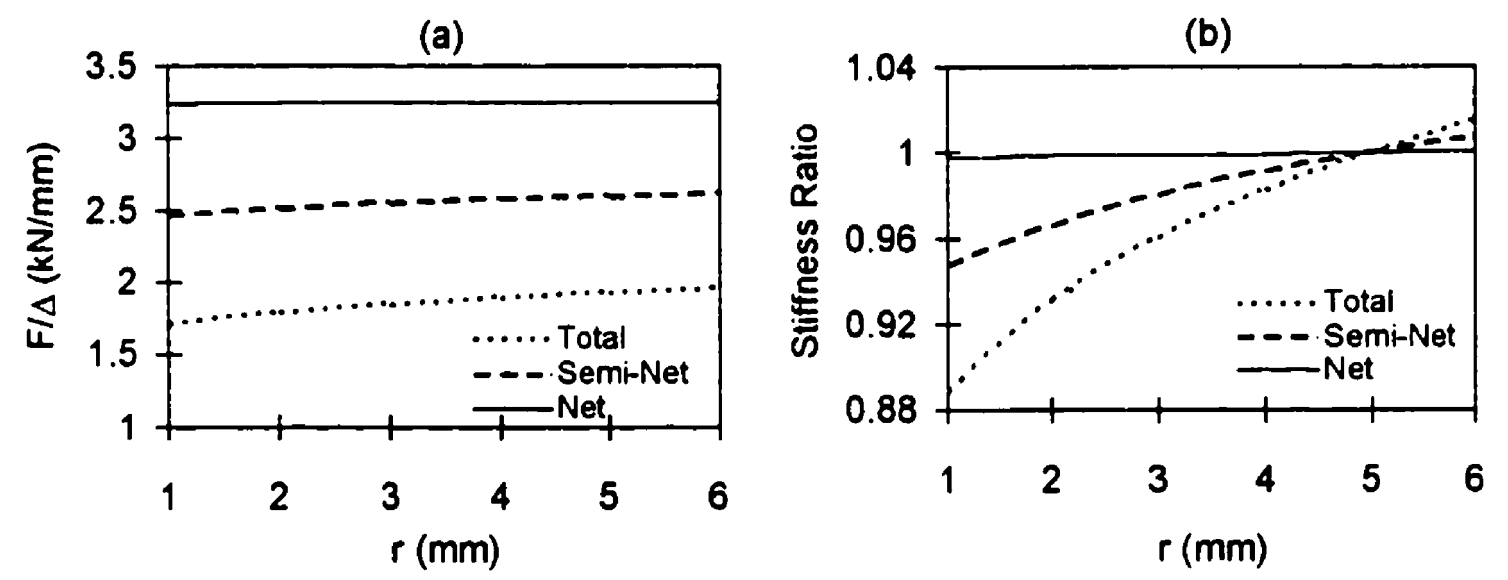

Figure 3.6 The effect of rollers radius on mid-span deflections for $E_{z:=}=10 \mathrm{GPa}$ and $v_{x=}=$ 0.25 . (a) Non-normalized results. (b) Normalized results.

The influence of $E_{z:}$ and $v_{x}$ values on the behavior of the beam are shown in Figs 3.7 and 3.8, respectively. The values in Fig. $3.7 \mathrm{~b}$ are normalized with respect to the stiffness value corresponding to $E_{z:=}=10 \mathrm{GPa}$. The stiffness value of $v_{x z}=0.25$ is also used for the normalization of values in Fig. $3.8 \mathrm{~b}$. The net mid-span deflection and the local deformation 
are selected as the dependent variables in these figures so that one can observe the variation of each of them separately. Figures $3.7 \mathrm{a}$ and $3.7 \mathrm{~b}$ show that the variation of $E_{z=}$ has significant effect on the local deformation, while the net mid-span deflection is not very sensitive to this parameters. The influence of $v_{x}$ as is shown in Figs $3.8 \mathrm{a}$ and $3.8 \mathrm{~b}$ is higher on net mid-span deflection than the local deformation, however the amount of variation is not of significant value.

(a)

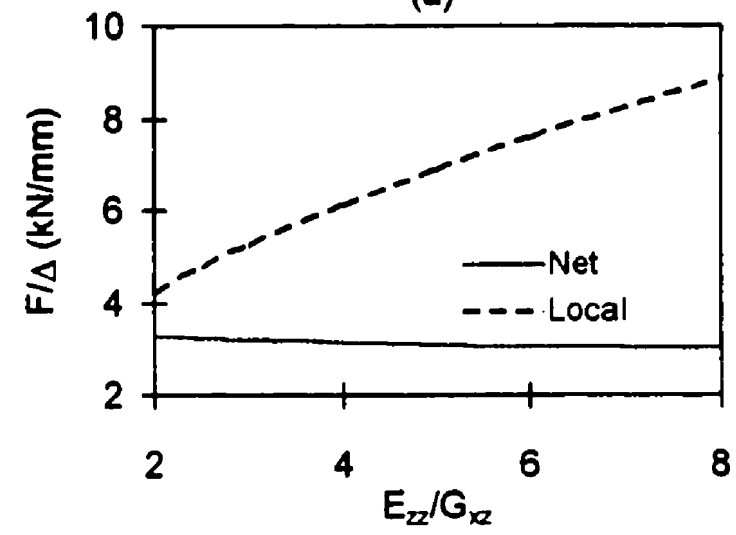

(b)

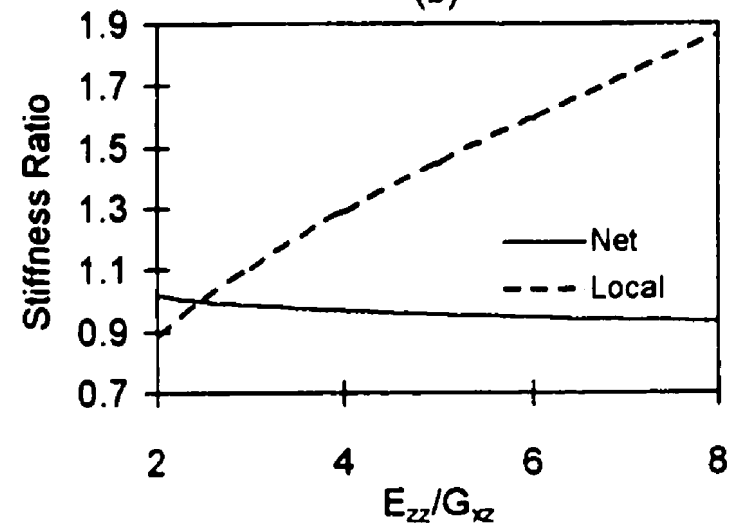

Figure 3.7 The effect of $E_{::}$on the mid-span deflections for $r=5 \mathrm{~mm}$ and $v_{x=}=0.25$.

(a) Non-normalized results. (b) Normalized results.

(a)

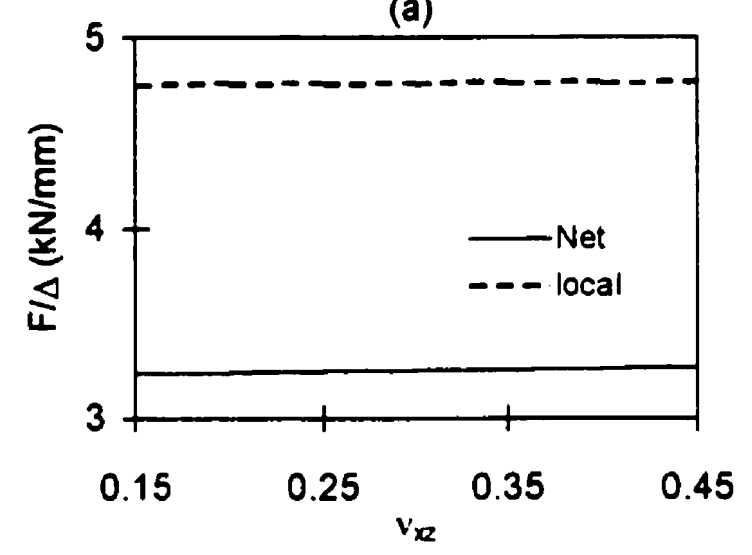

(b)

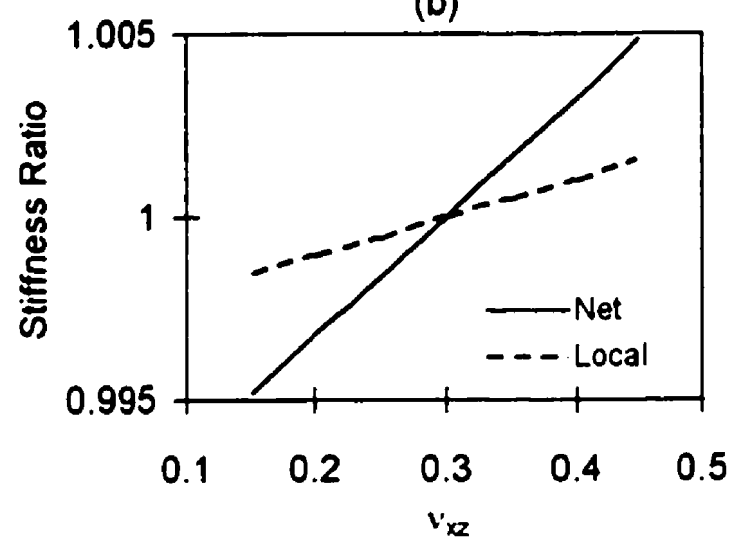

Figure 3.8 The effect of $v_{x z}=0.25$ on the mid-span deflections for $r=5 \mathrm{~mm}$ and $E_{z:}=10$ GPa. (a) Non-normalized results. (b) Normalized results. 
Recognizing that the application of the exact elasticity solution is too complicated to be considered as a practical tool for the evaluation of the VSM test results, one should rely on an approximate theory for this purpose. The investigation conducted in this section on beams with small $L / h$ and high anisotropy indicates that among the three mid-span deflections defined in Eqn (3.30), i.e. $\Delta_{N}, \Delta_{\mathrm{SN}}$ and $\Delta_{\mathrm{T}}$, only the net mid-span deflection $\left(\Delta_{N}\right)$ shows insignificant dependency to the parameters that usually are not accounted for in the approximate theories available for beams. Therefore, when the approximate theories are used, among the quantities defined in Eqn (3.30), the net mid-span deflection is expected to be the most accurately determined quantity. The application of two approximate beam theories will be presented in Chapter 5 . It will be shown that the solution based on these theories will be capable of accurately predicting the net mid-span deflection.

\subsection{Summary and conclusions}

The exact solution of a simply supported beam subjected to three-point bending was developed by the application of the theory of elasticity. The solution is different from the solutions available in the literature. The governing boundary conditions in the solution better represent the problem of the simply supported beam compared with those of existing solutions. The solution was then implemented into a computer program called ELAS-3P which was used to investigate the behavior of short beams with different geometry and material properties.

In summary the effect of parameters, such as the magnitude of the applied load, the radius of the loading nose and the supporting rollers, $E_{z:}$ and $v_{x=}$ on the response of a beam with $L / h=4$ and highly anisotropic material was investigated. A new terminology called the "net mid-span deflection" was defined. The investigation showed that the net mid-span deflection is not very sensitive to the above parameters. 
This finding is important, since for the VSM, approximate beam theories must be used. This is due to the fact that the exact elasticity solution accounts for the through-thethickness properties and is very complex. The insensitivity of the net mid-span deflection to parameters such as the radii of the loading nose and supports rollers and the throughthe-thickness properties is a promising sign that this quantity for all range of $L / h$ can be accurately predicted by the approximate beam theories. 


\section{Chapter 4}

\section{APPLICATION OF THE THROUGH-THE-THICKNESS INEXTENSIBILITY THEORY TO ORTHOTROPIC BEAMS}

The exact elasticity solution for an orthotropic beam was presented in the previous chapter. The solution provided is very useful for academic and research verification purposes. However, it is too complicated and lengthy for practical use. It requires the through-the-thickness elastic properties $\left(E_{z=}, v_{x}\right)$ of the material beside the longitudinal and the shear moduli. Therefore, the solution treats the flexural test as a four-unknown problem, requiring four independent tests and complicated data processing for the determination of the four elastic constants. A practical solution for the VSM must depend only on the longitudinal and shear moduli. As a result, the exact elasticity solution cannot be considered a practical means for this purpose. In this chapter we investigate the application of the through-the-thickness inextensibility (TTTI) theory developed by Jalali and Taheri (1998a, b, c) in which the effect of $E_{z: z}$ and $v_{x}$ is ignored. The theory is approximate in its nature, since it assumes that the beam is inextensible through its thickness; otherwise the theory involves no other approximation.

The through-the-thickness inextensibility theory was used by Jalali and Taheri (1998a, b, c) to solve the problems of simply supported laminated plates under cylindrical and planer bending. Their results showed very good agreement with the results obtained by Pagano $(1969,1970)$ and Jones (1970), who presented the exact elasticity solutions of the same problems for static and dynamic cases, respectively. The main advantage of the TTTI solutions provided by Jalali and Taheri $(1998 \mathrm{a}, \mathrm{b}, \mathrm{c})$ compared to the exact elasticity solutions is that they do not require the through-the-thickness properties of the materials. Furthermore, solutions based on the TTTI theory require less computational effort. On the other hand, the theory is different from many higher-order laminate theories [Whitney and Pagano (1970), Chow (1971), Whitney and Sun (1973), Lo et al. (1977) and Reddy 
$(1984,1989)]$ and the layerwise laminate theories [Robbins and Reddy (1993), Basar et. al. (1993) and Reddy (1989)] available in the literature, since it does not imply any presumption on the in-plane displacement components of the laminate. Instead, the engineering strain, $\gamma_{x}$ and $\gamma_{y z}$, are used for developing the through-the-thickness displacement field of laminates. These strains are defined as

$$
\begin{aligned}
& \gamma_{x}=\frac{\partial w}{\partial x}+\frac{\partial u}{\partial z} \\
& \gamma_{y z}=\frac{\partial w}{\partial y}+\frac{\partial v}{\partial z}
\end{aligned}
$$

where $u$ and $v$ are the in-plane displacements in the $x$ and $y$ directions, respectively, and $w$ denotes the out-of-plane displacement in the $z$ direction. If one assumes $w$ is constant through the thickness, the integration of Eqn (4.1) with respect to $z$ leads to a displacement field in the $x$ and $y$ directions as follows

$$
\begin{aligned}
& u=-z \frac{\partial w}{\partial x}+u^{0}+\int_{0}^{z} \gamma_{x=} d z \\
& v=-z \frac{\partial w}{\partial y}+v^{0}+\int_{0}^{z} \gamma_{y z} d z
\end{aligned}
$$

where $u^{0}$ and $v^{0}$ are the displacements at $z=0$. The assumption of a constant $\gamma_{x z}$ and $\gamma_{y z}$ through the thickness in the above equations leads to the solution of Mindlin (1951) for isotropic plates, and of Yang et al. (1966), Whitney and Pagano (1970) and Chow (1971), for laminated plates. The assumption of linear variation of $\gamma_{x}$ and $\gamma_{y z}$ leads to the solution of Whitney and Sun (1973) and by taking a parabolic function for $\gamma$, one obtains the solution of Reissner $(1945,1975)$ and Reddy (1984) for isotropic and laminated plates, respectively.

The solutions provided by Jalali and Taheri $(1998 \mathrm{a}, \mathrm{b}, \mathrm{c})$ are different from the above mentioned ones, since the exact form of $\gamma$ was found by solving the equilibrium differential 
equation(s), satisfying the boundary and the compatibility conditions. They presented the solutions for the multi-layer laminates. Their solution can be directly used for our singlelayer orthotropic beam in here. However, for the sake of clarity, the application of the TTTI theory to this problem is discussed in this chapter.

\subsection{Formulation for a beam subjected to harmonic loading}

Consider the beam shown in Fig. 4.1 with the $x y z$ coordinate system located at the center of the beam. The beam is subjected to a distributed harmonic load and has two simple supports at its two ends. It is composed of an orthotropic material with the principal material axes parallel to the $x y z$ coordinate system axes. Since we are only concerned with the variables in the $x$ direction, we omit the subscripts for simplicity. Therefore, $\sigma$ and $\varepsilon$ are the stress and strain in the $x$ direction and $\tau$ is the through-the-thickness shear stress in the $z y$ plane, respectively. The boundary conditions of the beam at the two ends are defined by

$$
x=0, L\left\{\begin{array}{l}
w=0 \\
\sigma=0
\end{array}\right.
$$

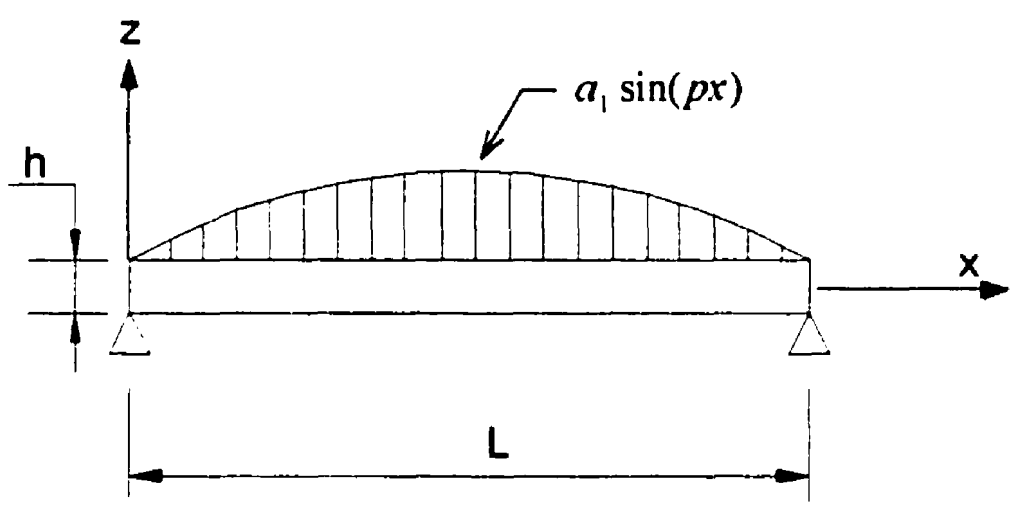

Figure 4.1 Simply supported beam subjected to harmonic loading.

Assuming the above problem is a plane stress problem, one can write the stress-strain relationship in the following form 


$$
\begin{aligned}
& \sigma=E \varepsilon \\
& \tau=G \gamma
\end{aligned}
$$

where $E$ is the longitudinal modulus in the $x$ direction and $G$ is the through-the-thickness shear modulus in the $z y$ plane.

The first derivative of Eqn (4.2a) with respect to $x$ gives the longitudinal strain, $\varepsilon$. Multiplying the result by the longitudinal modulus $(E)$, one can find the longitudinal stress as

$$
\sigma=-z E \frac{d^{2} w}{d x^{2}}+E \frac{d u^{0}}{d x}+\frac{E}{G} \int_{0}^{\delta_{0}} \frac{d \tau}{d x} d z
$$

Recognizing that there is no body force in the $x$ direction, the equilibrium condition in the $x$ direction is defined by

$$
\frac{d \sigma}{d x}+\frac{d \tau}{d z}=0
$$

Substituting Eqn(4.5) into Eqn(4.6) gives

$$
\frac{d \tau}{d z}=z E \frac{d^{3} w}{d x^{3}}-E \frac{d^{2} u^{0}}{d x^{2}}-\frac{E}{G} \int_{0}^{\sum_{0}} \frac{d^{2} \tau}{d x^{2}} d z
$$

The harmonic load is defined by

$$
q(x)=a_{n} \sin p x
$$

where $a_{n}$ is a constant indicating the amplitude of the load and

$$
p=\frac{n \pi}{L} \quad n=1,2,3, \ldots
$$

The solution of Eqn (4.7) for the load defined by Eqn (4.8) is of the form

$$
\begin{aligned}
& w=w_{0} \sin p x \\
& \tau=B \cos p x \\
& u^{0}=c \cos p x
\end{aligned}
$$

where $B$ is a function of $z$ but $w_{o}$ and $c$ are constants. Substituting Eqns (4.10) into Eqn (4.7) leads to 


$$
\frac{d B}{d z}=-z E w_{0} p^{3}+c E p^{2}+\frac{E}{G} p^{2} \int_{0}^{z} B d z
$$

To solve this differential equation, we use the Laplace transformation technique. Equation (4.11) after transformation becomes

$$
s \bar{B}-B(0)=-\frac{E w_{0} p^{3}}{s^{2}}+\frac{c E p^{2}}{s}+\frac{\beta \bar{B}}{s}
$$

where $\bar{B}$ is the $B$ function after transformation and $s$ is the variable in the transformed domain. Also

$$
\beta=\frac{E p^{2}}{G}
$$

Equation (4.12) after some manipulation can be written in the following form

$$
\hat{B}=\left[B(0)-\frac{E w_{0} p^{3}}{\beta}\right] \frac{s}{s^{2}-\beta}+\frac{E w_{0} p^{3}}{\beta s}+\frac{c E p^{2}}{s^{2}-\beta}
$$

The inverse Laplace transformation of this equation gives the $B$ function as

$$
B=\left[B(0)-\frac{E w_{0} p^{3}}{\beta}\right] \cosh (\sqrt{\beta} z)+\frac{E w_{0} p^{3}}{\beta}+\frac{c E p^{2}}{\sqrt{\beta}} \sinh (\sqrt{\beta} z)
$$

Equations (4.10) satisfy the boundary conditions of simple supports at the two ends of the beam defined by Eqn (4.3). To satisfy the condition of zero shear stress at the top and the bottom of the beam, it requires

$$
\begin{aligned}
& B(h / 2)=0 \\
& B(-h / 2)=0
\end{aligned}
$$

Applying these boundary conditions to Eqn (4.15) gives

$$
\begin{gathered}
B=w_{0} G p\left[1-\frac{\cosh (\sqrt{\beta} z)}{\cosh (\sqrt{\beta} h / 2)}\right] \\
c=0
\end{gathered}
$$

As a result 


$$
\begin{gathered}
\tau=w_{0} G p\left[1-\frac{\cosh (\sqrt{\beta} z)}{\cosh (\sqrt{\beta} h / 2)}\right] \cos (p x) \\
\sigma=\frac{w_{0} E p^{2}}{\sqrt{\beta}} \frac{\sinh (\sqrt{\beta} z)}{\cosh (\sqrt{\beta} h / 2)} \sin (p x)
\end{gathered}
$$

To determine the shear force along the beam, one must integrate Eqn (4.18) over the thickness of the beam. The result of the integration gives the following relationship between the shear force and the amplitude of the deflection

$$
V=w_{0} G p h\left[1-\frac{\tanh \eta}{\eta}\right] \cos (p x)
$$

where

$$
\eta=\sqrt{\beta} h / 2
$$

On the other hand from the mechanics of structures the following relation holds.

$$
\frac{d V}{d x}=-q
$$

Substituting Eqns (4.20) and (4.8) into Eqn (4.22), one can determine $w_{o}$ as

$$
w_{0}=\frac{a_{n}}{p^{2} G h} \frac{1}{1-\frac{\tanh \eta}{\eta}}
$$

The longitudinal and the shear stresses can now be written in their final form by substituting Eqn (4.23) into Eqns (4.19) and (4.18), respectively. Thus

$$
\begin{gathered}
\sigma=\frac{1}{2} \times \frac{a_{n} \phi^{2}}{\eta-\tanh (\eta)} \times \frac{\sinh (2 \eta z / h)}{\cosh (\eta)} \sin (p x) \\
\tau=\frac{1}{2} \times \frac{a_{n} \phi}{\eta-\tanh (\eta)}\left[1-\frac{\cosh (2 \eta z / h)}{\cosh (\eta)}\right] \cos (p x)
\end{gathered}
$$

where

$$
\phi=\sqrt{E / G}
$$




\subsection{Numerical investigations}

In this section we compare the solution provided in the previous section with the exact elasticity solution of Section 3.1. The comparison is made for a distributed half-sine load $(n=1)$ on the top of the beam and a concentrated load at mid-span. In both cases the properties of the beam material are assumed to be

$$
\begin{array}{ll}
E_{x x}=200 \mathrm{GPa} & E_{z=}=10 \mathrm{GPa} \\
G_{x=}=4 \mathrm{GPa} & U_{x=}=0.25
\end{array}
$$

which represents a highly anisotropic material.

\subsubsection{Half-sine load}

Consider the beam shown in Fig. 4.1. The exact elasticity solution of this beam is the same as what was presented in Section 3.1 when the amplitude of the bottom load is set to zero. The solution was implemented in a program called ELAS-SIN. The program's code is presented in Appendix B. The computer program for the TTTI solution is called "TTTISIN" and its code is presented in Appendix C. Both programs were written in the Qbasic programming language.

First we compare the deflections of the two solutions for beams with different $L / h$. Since in the exact solution the displacement in the $z$ direction changes over the thickness of the beam, we determine the average displacement of the section. For this, we integrate the second equation of Eqn (3.8) over the thickness, and divide the result by the thickness of the beam. The result is as follows

$$
w_{a v}=\sin (p x) \sum_{i=1}^{+} \frac{A_{1}}{h m_{i}}\left(\bar{S}_{13} m_{1}-\frac{\bar{S}_{33}}{m_{1}} p^{2}\right)\left[\exp \left(\frac{m_{i} h}{2}\right)-\exp \left(-\frac{m_{i} h}{2}\right)\right]
$$

The distribution of $w$ normalized with respect to $w_{a v}$ for a beam with $L / h=4$ is shown in Fig. 4.2. As the figure shows the displacement in the $z$ direction increases from the bottom of the beam to the top. This is due to the through-the-thickness deformation of the beam. This phenomenon is ignored in the TTTI theory, and as a result the deflection at each 
section represents the $z$ direction displacements of all the points in the section. The comparison of the deflections obtained from the two solutions for beams with different $L / h$ is presented in Fig. 4.3. The figure depicts the deflections obtained from TTTI after normalization with respect to those obtained from the exact elasticity solution. As the figure shows, the maximum margin of error for the deflections obtained from the TTTI solution is about $0.2 \%$. This indicates that the TTTI theory is quite accurate in predicting the overall deflections of the beams. The existing error vanishes when $L / h$ increases.

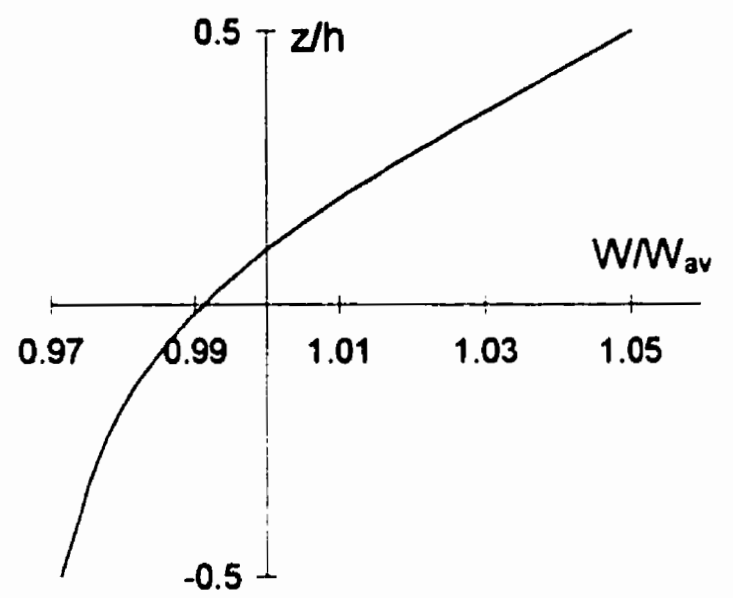

Figure 4.2 Distribution of the displacement in $z$ direction over the thickness of the beam in exact elasticity solution.

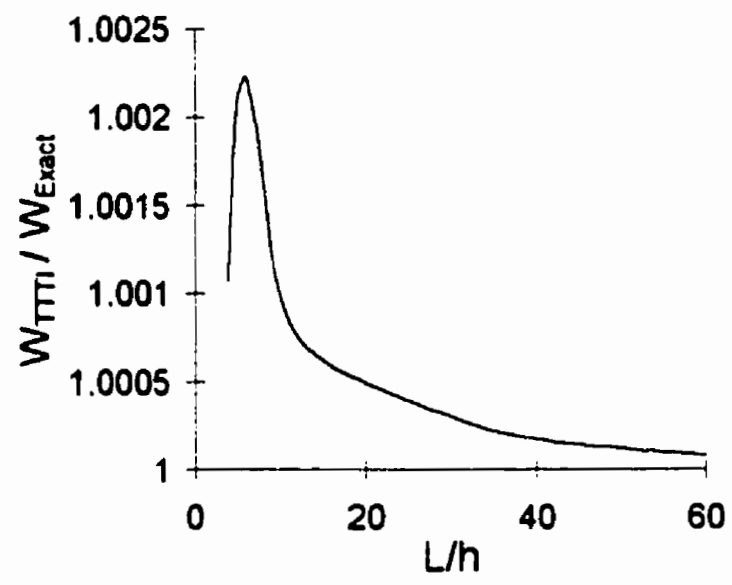

Figure 4.3 Comparison of the deflections of the TTTI and the exact elasticity solution. 
The minute difference between the exact and the TTTI solutions is due to the TTTI assumption that the beam is inextensible through its thickness. The over estimation of the deflections can be explained using the principle of energy. The energy due to the applied external load is transferred into internal energy comprising of various components. For the present case study the constituents are due to $\varepsilon_{x x} \sigma_{x x}, \gamma_{x} \tau_{x=}$ and $\varepsilon_{z z} \sigma_{z z}$. The first two constituents correspond to the overall deflection of the beam and the last corresponds to the through-the-thickness deformation. Ignoring the through-the-thickness deformation, and therefore the corresponding energy, leads to an over estimation of the energy associated with the overall deflection. This, in turn, results to an over estimation of the overall deflections. Note that the exact solution converges to the TTTI solution when the through-the-thickness stiffness of the beam increases.

The comparison of the stresses obtained from the TTTI and the exact elasticity solution is shown in Fig. 4.4. Since the discrepancy of the results increases as $L / h$ decreases, the comparison is presented for a beam with $L / h=4$ which is considered to be the most severe case in the VSM. The following normalized quantities have been used in connection with the figure

$$
\bar{\sigma}=\frac{\sigma\left(\frac{L}{2}, z\right)}{a_{1}} \quad \bar{\tau}=\frac{\tau(0, z)}{a_{1}}
$$

where $a_{1}$ is the amplitude of the half-sine load. As the figure shows, the TTTI solution agrees very well with the exact elasticity solution. These results in conjunction with those obtained for the deflections confirms the adequacy of the TTTI theory for predicting the behavior of simply supported beams under distributed loads. 
(a)

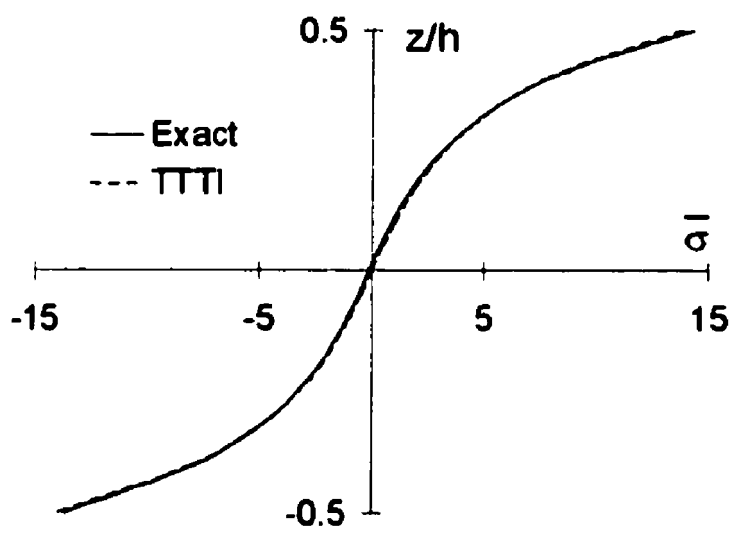

(b)

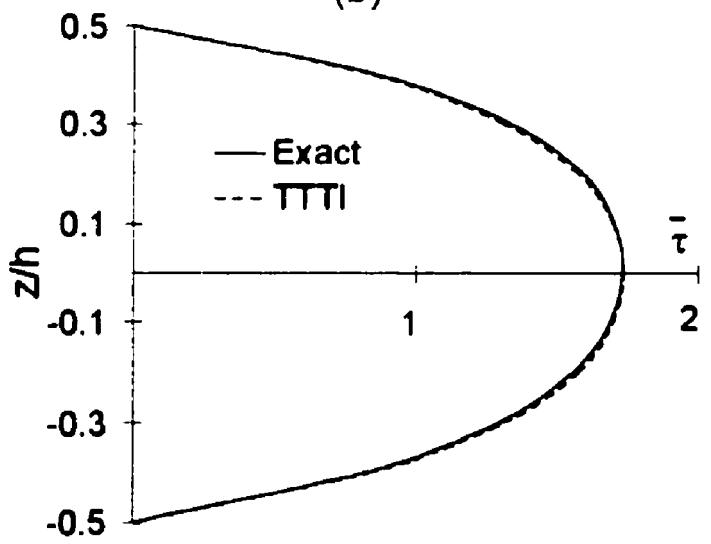

Figure 4.4 Comparison of the stresses obtained from the TTTI and the exact elasticity solution for a beam with $L / h=4$. (a) Longitudinal stresses. (b) Shear stresses.

\subsubsection{Concentrated load at mid-span}

To investigate the adequacy of the TTTI theory for predicting the response of beams subjected to a concentrated load at mid-span, consider the beam shown in figure 4.5. The thickness of the beam and the diameter of the loading nose are selected to represent the actual dimensions of a real FRPC specimen flexural test. The distribution of the stresses beneath the loading nose is assumed to follow Eqns (3.14) through (3.16). The loading nose is assumed to be steel with $E=200 \mathrm{GPa}$. The elastic modulus of the beam in the $z$ direction is also used for the determination of the contact behavior. The Fourier transformation of the load is expressed by Eqn (3.18). Replacing $L^{\prime}$ with $L$ in Eqns (3.23) and (3.24), the series coefficients are obtained from the following relations

$$
\begin{gathered}
a_{n}=(-1)^{\frac{n-1}{2}} \frac{2 F}{L} \frac{\cos (p \bar{b})}{1-4\left(\frac{p \bar{b}}{\pi}\right)^{2}} \quad \text { for } \frac{p \bar{b}}{\pi} \neq \frac{1}{2} \\
a_{n}=(-1)^{\frac{n-1}{2}} \frac{\pi F}{2 L} \quad \text { for } \frac{p \bar{b}}{\pi}=\frac{1}{2}
\end{gathered}
$$

where $\bar{b}$ is half of the contact length and $p$ is defined by Eqn (4.9). The exact elasticity solution and the TTTI solution of the beam are obtained by the superposition of the result 
obtained for the various series components. The procedures for the two solutions were implemented in two different computer programs, called ELAS-CON and TTTI-CON, and are documented in Appendices D and E, respectively. The results obtained from the analyses of beams with different $L / h$ are discussed next.

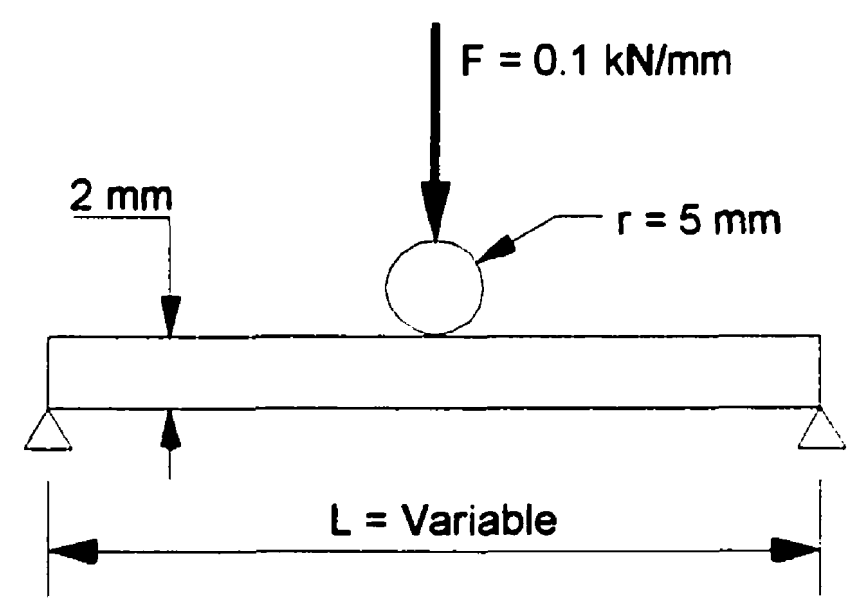

Figure 4.5 Configuration of the beams subjected to a concentrated load.

Since the concentrated load produces a significant amount of through-the-thickness displacement, the average deflection at mid-span from the exact solution cannot represent the overall deflection of the beam. A more representative quantity for the comparison with the deflection obtained from the TTTI solution is deemed to be the deflection obtained at the bottom surface of the beam. This comparison for beams with different $L / h$ is presented in Fig. 4.6. As the curve identified by TTTI shows, the overestimation of the deflection in this case is much higher than the previous case when a half-sine load was considered. The figure also presents a curve identified by "TTTI-modified". Definition of this curve will be discussed later. In Figs $4.7 \mathrm{a}$ and $4.7 \mathrm{~b}$ the comparison is made for a beam half length with $L / h=4$. In these figures $\bar{y}$ is the distance measured from mid-span. The figures show that the difference of the results from the two solutions is significant in the vicinity of the concentrated load, while the difference vanishes as the distance from the concentrated load increases. 


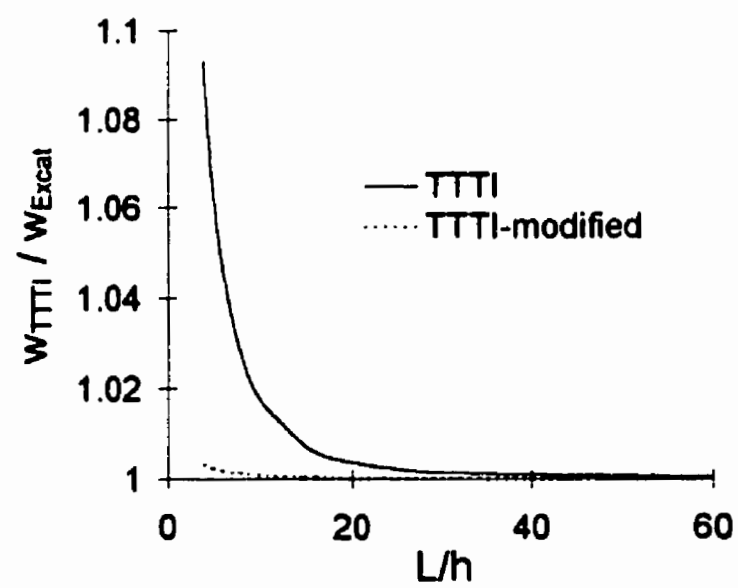

Figure 4.6 Comparison of mid-span deflections determined from the TTTI and the exact elasticity solutions.
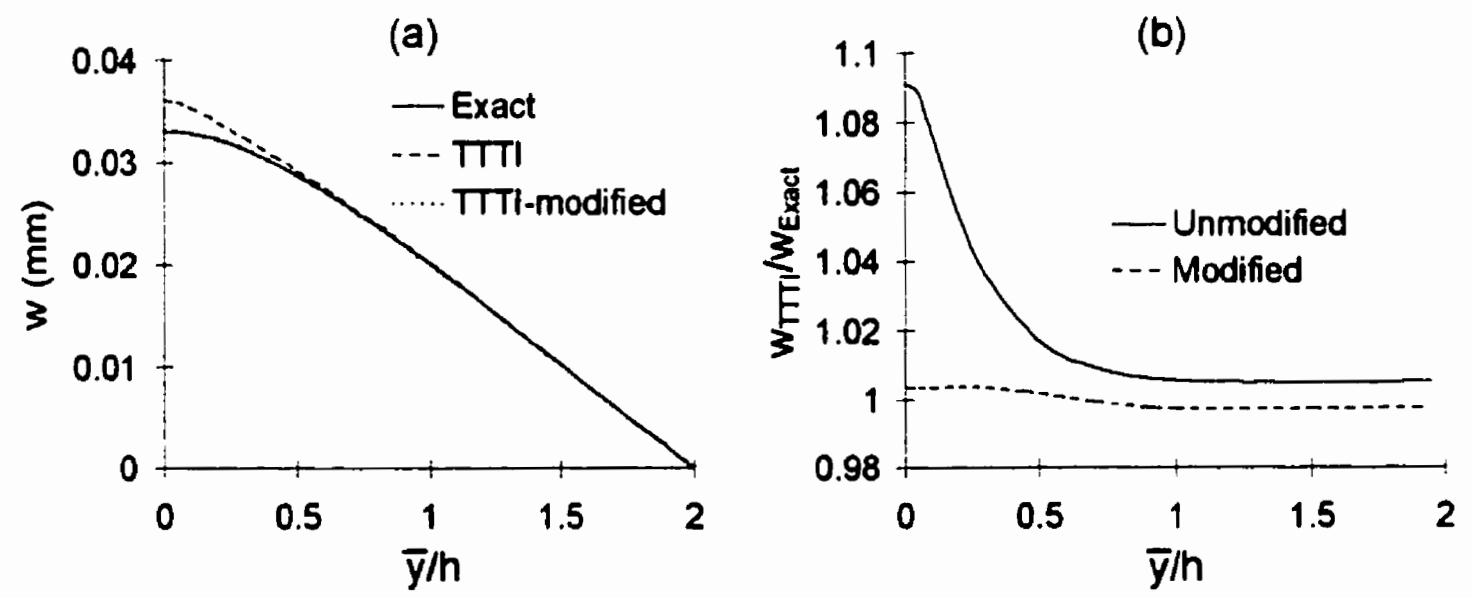

Figure 4.7 Comparison of deflections determined from the TTTI and the exact elasticity solutions for beam with $L / h=4$.

The longitudinal stresses at the top surface of the beam at the mid-span are significantly affected by the presence of the concentrated load. As shown in Fig. 4.8, this stress is several times higher than the stress at the bottom of the beam in the same section. The local effect of the concentrated load rapidly vanishes as the distance from the contact point increases. This is illustrated in Fig. 4.8 by the fluctuation of the top longitudinal stress in 
the vicinity of the contact point and the fact that its value approaches the bottom longitudinal stress at a distance of about $\bar{y}=h$. On the other hand, the variation of the bottom longitudinal stress along the beam is quite smooth and is not affected by the concentrated load. This is due to the fact that the bottom surface has sufficient distance from the contact point of the load, so that the local effect of the load has completely decayed at that distance.

The longitudinal stress obtained from the TTTI solution is also illustrated in Fig. 4.8. Similar to the deflections, the stresses at the bottom of the beam from the two solutions do not agree very well. The difference becomes significant at the vicinity of the mid-span. This difference cannot be related to the local effect of the concentrated load, since as stated earlier the bottom surface has sufficient distance from the contact point. The reason for such differences and its remedy will be discussed next.

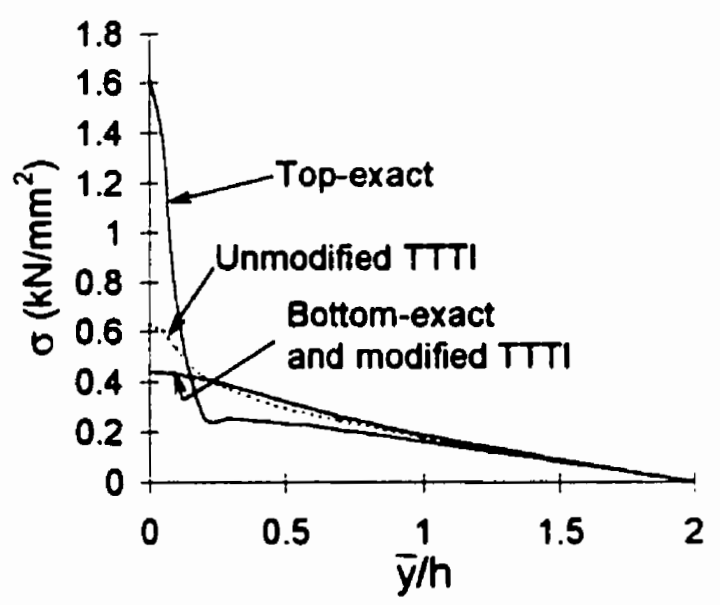

Figure 4.8 Comparison of stresses determined from the TTTI and the exact elasticity solutions for beam with $L / h=4$.

Figure 4.9 shows the distribution of $\sigma_{:=}$at three elevations through the thickness of the beam. These distributions were determined by the exact solution. The curve corresponding to each elevation is normalized in such a way that the area underneath represents $0.1 \mathrm{kN}$. 
As the figure shows, the heavily concentrated stress distribution on the top surface $(z / h=$ 0.5 ) becomes smoother as the distance from the top surface increases. The assumption of inextensibility, which is the basis of the TTTI method, does not account for this gradual distribution of the load, and as a result, it leads to an overestimation of the axial stresses and the deflections in the vicinity of the mid-span. To account for the gradual distribution of the load in the TTTI method, one must distribute the concentrated load over a slightly wider length. For this purpose, we assume the distribution of load to be based on the following relation, which is also illustrated in Fig. 4.9.

$$
q=\frac{F}{8.75 \bar{b}}[1+\cos (\pi \bar{y} / \bar{b})]^{4}
$$

where $\bar{b}$ is half of the width assumed for the distribution of the load. The value of $\bar{b}$ depends on the degree of anisotropy of the beam material and increases as the anisotropy increases. Jalali and Taheri (1998a) reported that for most practical purposes this value falls between $1.3 h$ and 1.6h. The curve in Fig. 4.9 is drawn for $\bar{b}=1.45 h$.

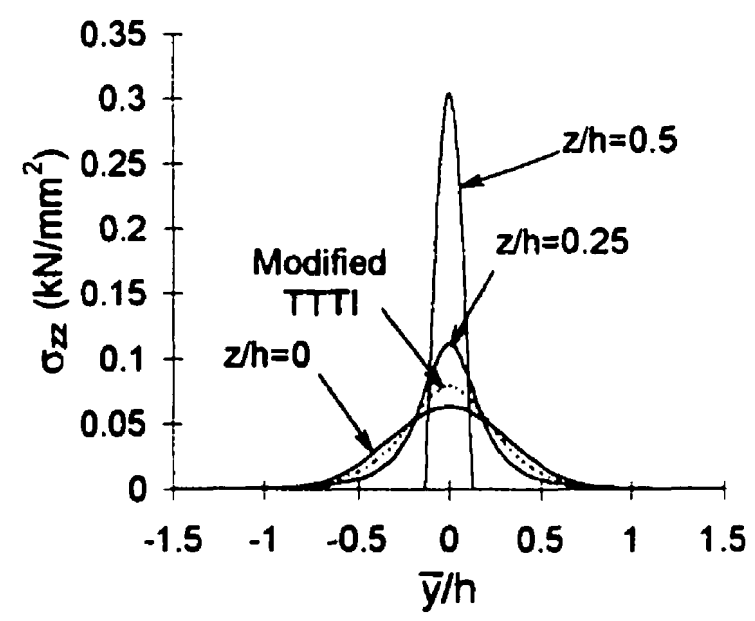

Figure 4.9 Distribution of the through-the-thickness normal stress at various levels of a beam with $L / h=4$.

The results of the calculations based on the modified distribution (Eqn (4.31)), as presented by lines labeled "modified TTTI" in Figs 4.6 through 4.8, agree very well with 
those of the exact solution. The distribution of the stresses on a section at a distance equal to the thickness of the beam from the mid-span is also shown in Fig. 4.10. The figure compares the stresses obtained from the TTTI solution based on the modified distribution and those from the exact solution. The agreement of the stresses except for a small part close to the top of the beam is very good. The small discrepancy between the results of the two solutions vanishes as the distance from the concentrated load increases. In summary the good agreement between the results obtained from the modified TTTI solution and those of the exact solution confirms the method of load distribution as discussed.
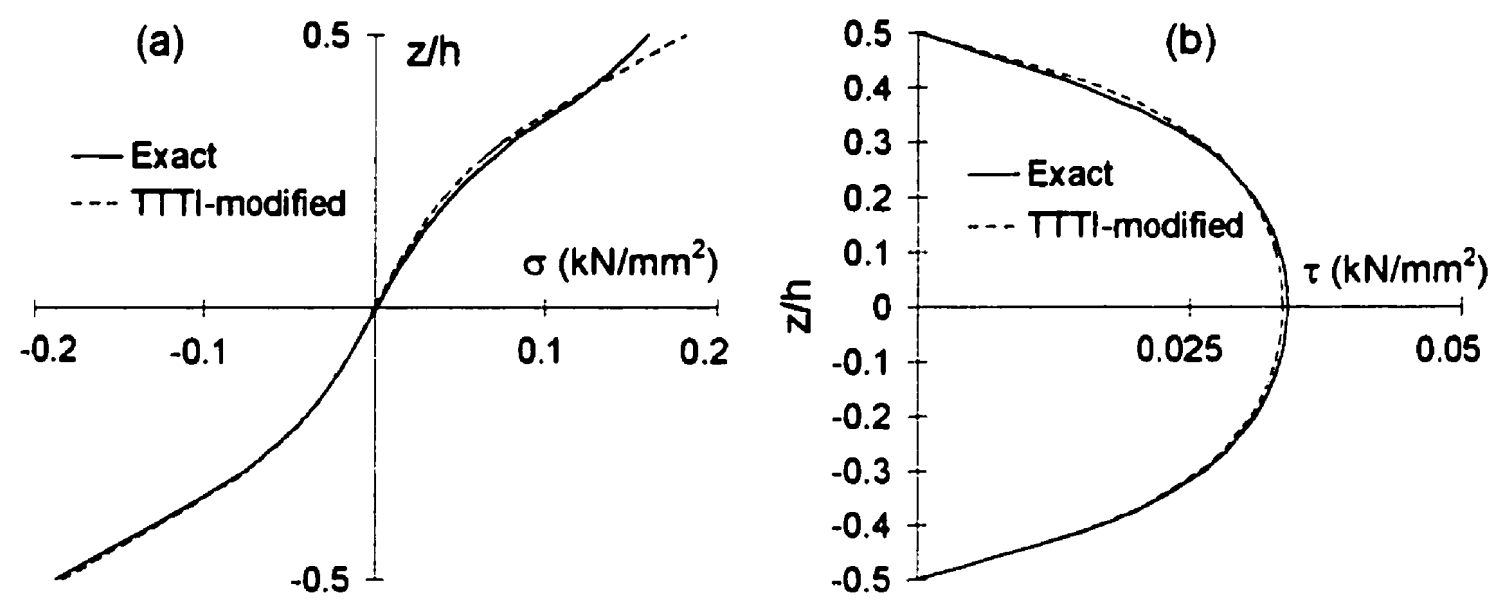

Figure 4.10 Comparison of the stresses obtained from the TTTI and the exact elasticity solutions. (a) Longitudinal stresses. (b) Shear stresses.

\subsection{Summary and conclusions}

The through-the-thickness inextensibility (TTTI) theory proposed by Jalali and Taheri (1998a, b, c) was used to find a solution for a simply supported orthotropic beam. The solution was used for the determination of the stresses and the deflections of a highly anisotropic beam under a half-sine distributed load and a concentrated load. The results were compared with those obtained by the exact elasticity solution discussed in Chapter 3 . The agreement between the results obtained from the two solutions was excellent for the half-sine load. It was also shown (Fig. 4.9) that the transfer of the concentrated load to the 
beam occurred gradually. As a result, to compensate for this delay in TTTI method, the concentrated load should have been distributed over a wider length than the one predicted by contact law. A new relation was proposed for this distribution. In the concentrated load case, the TTTI solution was incapable of predicting the accurate deflections and the stresses in the vicinity of the load. However, the method provided accurate results for the bottom and the sections farther than a distance equal to the thickness of the beam from the concentrated load when the proposed distribution was employed.

The solution based on the TTTI theory requires only the longitudinal and the through-thethickness shear moduli ( $E_{x x}$ and $G_{x x}$ ) of the material for predicting the behavior of an orthotropic beam. In contrast, the exact elasticity solution additionally requires $E_{=z}$ and $v_{x z}$. This characteristic of the TTTI solution makes it a practical tool for use with the VSM to determine the longitudinal and the through-the-thickness shear moduli of the FRPC specimens. 


\section{Chapter 5}

\section{DEVELOPMENT OF THE VARYING-SPAN METHOD}

The exact solution of a simply supported beam subjected to three-point bending was presented in Chapter 3. The solution assumed the beam was under a state of plane stress, and as a result, $E_{x x}, E_{z=}, G_{x=}, v_{x=}$ were the elastic properties required in the formulation provided. A new terminology, called the net mid-span deflection, was also introduced. It was shown that this quantity was not sensitive to the through-the-thickness properties of the beam $\left(E_{z:}\right.$ and $\left.v_{x z}\right)$ and the contact phenomenon.

In chapter 4, we employed the theory of the through-the-thickness inextensibility (TTTI) to predict the behavior of a simply supported beam. It was shown that the TTTI solution, which does not consider the effect of $E_{z:}$ and $\nu_{x=}$, provided accurate results for the overall behavior of the beam. For beams subjected to a concentrated load, the TTTI solution overestimated the mid-span deflection. This shortfall, however, was resolved by using Eqn (4.31), thereby applying the concentrated load on a finite width. The deflections predicted by TTTI solution were then in very good agreement with those obtained by the exact elasticity solution for the bottom of the beam.

These findings assure that the overall behavior of a simply supported beam can be accurately predicted by knowing only the longitudinal and the through-the-thickness shear moduli. The level of contribution of these two elastic moduli in the response of the beam depends on the span-to-depth ratio $(L / h)$ of the beam. The effect of the shear modulus for beams with small $L / h$ is quite significant, while its effect reduces rapidly as $L h$ increases.

On the other hand for a given $L / h$, the influence of the shear modulus becomes more significant when the ratio of the longitudinal-to-shear moduli $(E / G)$ of the material increases. 
In three-point bending tests the net mid-span deflection is one of the quantities that is controlled by the overall behavior of the beam. This quantity can easily be recorded during the test and is selected for the characterization of the test specimen. Recognizing that FRPC often have large $E / G$ values, the net mid-span deflection measured for specimens with small and average $L / h$ carries a significant amount of shear deformation. This characteristic brings the possibility of simultaneous evaluation of both the longitudinal and shear moduli of FRPC by changing the effect of shear modulus in the three-point bending test. This can easily be accomplished by conducting tests on specimens with different $L \boldsymbol{h}$. We call the test method the "Varying Span Method" (VSM), due to the fact that in practice variable $L / h$ can easily be produced by only changing the span of the test.

The distribution of the concentrated load over a finite width according to Eqn (4.31), as was shown in Chapter 4, produces good results for the deflections and also the stresses on the bottom surface of the beam and at any location farther than " $h$ " from the concentrated load. However, further investigation has shown that even a simple uniform distribution of the concentrated load produces acceptable results, as long as the only concern is the deflection values. In Fig. 5.1b the applied mid-span load and the reactions are distributed uniformly over a length equal to $2 \bar{b}$. This configuration is used to predict the net mid-span deflection of the beam shown in Fig 5.1 a. Note that we are looking for solutions which do not consider the effects of $E_{z=}$ and $v_{x=}$. Otherwise, for the exact elasticity solution, the distribution of the concentrated load must be based on the contact behavior of the contacting bodies (i.e. interface of the beam with rollers and loading nose). Therefore, the suggested technique of distributing the load and reaction forces over wider length should not be applied.

For simultaneous evaluation of the longitudinal and through-the-thickness shear moduli of the material, one needs a solution that can adequately predict the response of a beam under three-point bending and requires only the two elastic constants. The solution based 
on TTTI theory, as was presented in Chapter 4 , has the required characteristic. The solution based on this theory for a beam with two simple supports at its two ends and under a concentrated load at mid-span was presented in Section 4.1. However, it cannot be used directly, since in the VSM, the supports are not located at the two ends of the beam. Therefore, the appropriate solution and the procedure for determining the longitudinal and the shear moduli from the test results are presented in this chapter.
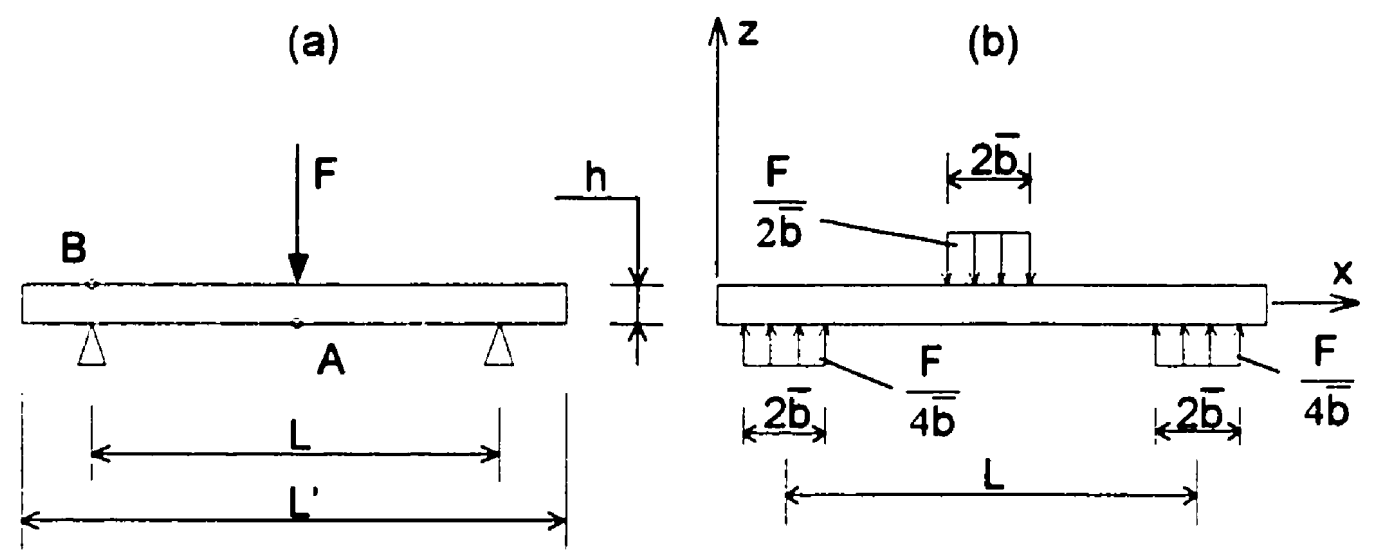

Figure 5.1 Representation of the mid-span concentrated load and the reactions with uniformly distributed load.

As was discussed earlier, among the various available higher-order theories proposed for the laminated structures, the first-order theory is the simplest one and provides satisfactory results for the deflections. For the beam type problem, this theory is identical to Timoshenko beam theory. The application of this theory to the VSM is also discussed in this chapter. The solution based on Timoshenko beam theory is much simpler than the one provided by TTTI method and will be widely used later.

Notice that we denote the $E_{x x}$ and $G_{x z}$ by $E$ and $G$ in the formulations. This is due to the fact that these two quantities are the only elastic constants in the formulation used for the VSM. Therefore, dropping the subscripts will not produce any confusion, while it provides some simplicity in the formulations. The subscripts, however, will be added wherever 
there is the possibility of confusion between these quantities and the other elastic constants.

\subsection{The application of the through-the-thickness inextensibility (TTTI) theory}

Consider the beam shown in Fig. 5.1b with the total length of $L^{\prime}$ and two imaginary simple supports at its two ends. The existence of the two imaginary supports enables one to use the TTTI solution for the harmonic load condition presented in section 4.1. However, since the loads at the top and the bottom of the beam are in equilibrium, the imaginary supports do not carry any forces. As a result, the solution provides the correct answers for the beam shown in Fig. 5.1b except that it carries a rigid body movement equal to the vertical displacement of the points located at the real supports $\left(w_{D}\right)$. This problem can easily be resolved by deducting $w_{D}$ from the deflection values.

To obtain the coefficients of the Fourier series for the loads shown in Fig 5.1b, we substitute the loads in Eqn (3.21). With some manipulation the following equation is derived

$$
a_{n}=(-1)^{\frac{n-1}{2}} \frac{2 F}{n \pi \bar{b}} \sin (p \bar{b})[1-\cos (p L / 2)] \quad n=1,3,5,7, \ldots
$$

where

$$
p=\frac{n \pi}{L^{\prime}} \quad n=1,3,5,7, \ldots
$$

The deflections along the beam for a single term of the Fourier series are defined by Eqn (4.10a). Substituting Eqns (5.1) and (4.23) into Eqn (4.10a) and superposing for all the series terms, one reaches the following equation

$$
w=\frac{2 F}{G b h \pi \bar{b}} \sum_{n=1}^{\infty} \frac{(-1)^{\frac{n-1}{2}}}{n p^{2}\left(1-\frac{\tanh \eta}{\eta}\right)} \sin (p \bar{b})[1-\cos (p L / 2)] \sin (p x)
$$


Since the deflection values obtained from this equation carry the rigid body movement of $w_{B}$, one must deduct $w_{B}$ from the values obtained from this equation. For the mid-span deflection, $\Delta$, one can write

$$
\Delta=w_{A}-w_{B}
$$

where $w_{A}$ and $w_{B}$ are the deflection values at points $A$ and $B$ obtained from Eqn (5.3), respectively. Substituting $w_{A}$ and $w_{B}$ from Eqn (5.3) into Eqn (5.4) gives

$$
\Delta=\frac{F}{G b h} f
$$

or after rearranging

$$
\frac{F}{\Delta}=\frac{G b h}{f}
$$

where $f$ is defined by the following equation

$$
f=\frac{2}{\pi \bar{b}} \sum_{n=1}^{\infty} \frac{1}{n p^{2}\left(1-\frac{\tanh \eta}{\eta}\right)} \sin (p \bar{b})[1-\cos (p L / 2)]^{2}
$$

The left hand side of Eqn (5.6) represents the flexural stiffness of the beam and can be determined from the slope of the load-deflection curve recorded from the flexural test. Since $E$ and $G$ are the only unknowns in Eqn (5.6), one needs two independent equations for determining the two unknowns. The data required for these equations can be obtained by conducting two flexural tests on specimens with different $L / h$. Using Eqn (5.6), one can correlate the flexural stiffness values obtained from the two tests as

$$
\frac{K_{1}}{K_{2}}=\frac{b_{1} h_{1} f_{2}}{b_{2} h_{2} f_{1}}
$$

where $K$ is the flexural stiffness defined by the left hand side of Eqn (5.6), and the subscripts $l$ and 2 correspond to the two different tests with different $L / h$. The only unknown in Eqn (5.8) is the $E / G$ value. This quantity, which determines $f_{l}$ and $f_{2}$, can be easily calculated by a trail and error procedure. For this, one may determine the right hand side of Eqn (5.8) by assuming different values of $E / G$ until the equality of the equation is 
satisfied. The final $E / G$ is then used to determine the value of $G$ by applying Eqn (5.6) to either test data. The value of $E$ is determined by multiplying $G$ by $E / G$.

A graphical solution of Eqn (5.8) is presented in Fig 5.2. Figure 5.2a presents the solution for the cases that specimens with $L / h=7$ and 30 are used. The solution for the cases that specimens with $L / h=5$ and 20 are used is presented in Fig. 5.2b. In each figure three curves representing different values of $\bar{b} / h$ are provided. To use these curves, one must first obtain the quantity of the abscissa from the test data of the two specimens with specified $L / h$ values. The $E / G$ value can then be easily obtained from the corresponding curve when the value of $\bar{b} / h$ is known. Determination of $\bar{b} / h$ will be discussed in the next chapter.
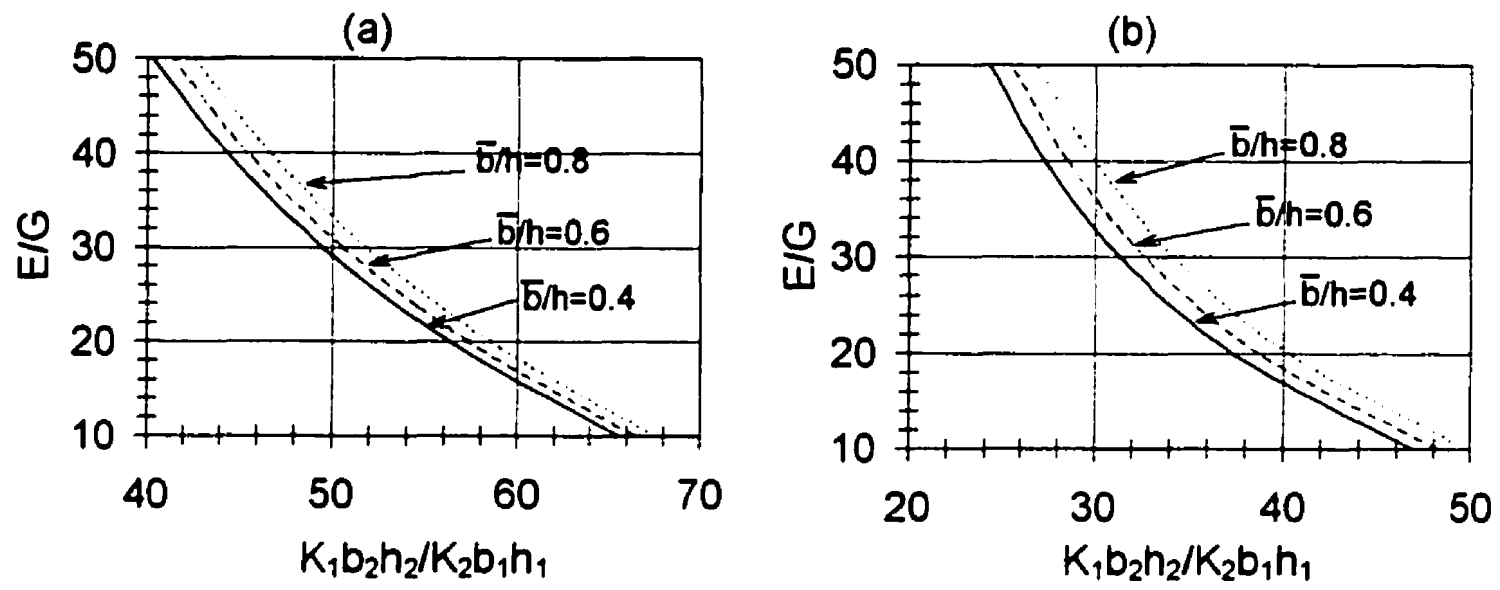

Figure 5.2 Graphic representation of Eqn (5.8). (a) For specimens with $L / h=7$ and 30. (b) For specimens with $L h=5$ and 20.

One may, obviously, question the reliability of the $E$ and $G$ values that are established based on only two tests. To increase the reliability of the results, one can perform more tests on specimens with different $L / h$. As a result, one will obtain $\mathrm{N}(\mathrm{N}-1) / 2$ different values of $E$ and $G$, where $\mathrm{N}$ is the total number of tests conducted on beams with different $L / h$. Special statistical procedures will then be needed to determine the values of the most probable $E$ and $G$. 
Alternatively, a simpler approach can be adopted to establish reliable values. One can use two sets of specimens. Each set will have several specimens with the same $L / h$, while the two sets will have different $L / h$. The $K / b h$ value for each $L / h$ will, then, be determined by averaging the values of the different specimens in the same set. As a result, the outcome will be statistically more reliable.

\subsection{The application of Timoshenko beam theory}

This theory assumes that the deflection of a beam comprises of two parts: one due to bending moment and the other due to shear forces. These quantities can be calculated separately. Therefore, for the deflection at mid-span one can write

$$
\Delta=\Delta_{\text {Flexural }}+\Delta_{\text {shear }}
$$

For the beam shown in Fig. 5.1b $\Delta_{\text {Flenural }}$ and $\Delta_{\text {Shear }}$ are determined from the following equations, respectively

$$
\begin{gathered}
\Delta_{\text {Newural }}=\frac{F}{48 E I}\left(L^{3}-2 L \bar{b}^{2}+\bar{b}^{3}\right) \\
\Delta_{\text {shear }}=\frac{3 F}{10 A G}(L-1.5 \bar{b})
\end{gathered}
$$

Therefore

$$
\Delta=\frac{F}{48 E I}\left(L^{3}-2 L \bar{b}^{2}+\bar{b}^{3}\right)+\frac{3 F}{10 A G}(L-1.5 \bar{b})
$$

where $I$ and $A$ are the moment of inertia and the cross section area of the beam, respectively. Ignoring the contribution of the shear in the deflection of the beam, Eqn (5.10) should express the total deflection. As a result, the $E$ value in the equation will be smaller than its real value when based on the total deflection. We call this quantity the "apparent modulus of elasticity" and denote it by $E^{\prime}$. Substituting $\Delta$ instead of $\Delta_{\text {fexural }}$ in Eqn (5.10) and rearranging the equation, one can express $E^{\prime}$ as

$$
E^{\prime}=\frac{F}{\Delta} \frac{L^{3}-2 L \bar{b}^{2}+\bar{b}^{3}}{48 I}
$$


In a flexural test the value of $F / \Delta$ can be obtained from the slope of the recorded loaddeflection curve. It is important to note that $E^{\prime}$ is not a constant value. In fact it carries the shear effect, and as a result, is a function of $L / h$ of the specimens. The value of $E^{\prime}$, however, approaches $E$ as $L / h$ becomes large.

Dividing both sides of Eqn (5.12) by $\frac{\left(L^{3}-2 L \bar{b}^{2}+\bar{b}^{3}\right)}{48 I}$ and setting $I=b h^{3} / 12$ and $A=b h$ gives

$$
\frac{\Delta}{F} \times \frac{4 b h^{3}}{\left(L^{3}-2 L \bar{b}^{2}+\bar{b}^{3}\right)}=\frac{1}{E}+1.2 \frac{L-1.5 \bar{b}}{L-\frac{2 \bar{b}^{2}}{L}+\frac{\bar{b}^{3}}{L^{2}}} \times \frac{1}{G}\left(\frac{h}{L}\right)^{2}
$$

The left hand side of this equation is the reciprocal of $E^{\prime}$. Defining $J$ as

$$
J=1.2 \frac{L-1.5 \bar{b}}{L-\frac{2 \bar{b}^{2}}{L}+\frac{\bar{b}^{3}}{L^{2}}}
$$

one reaches the following equation

$$
\frac{1}{E^{\prime}}=\frac{1}{E}+\frac{1}{G} \mathcal{J}\left(\frac{h}{L}\right)^{2}
$$

This equation is the equation of a straight line in which $J(h / L)^{2}$ and $1 / E^{\prime}$ are the independent and dependent variables, respectively. An schematic view of the equation is illustrated in Fig. 5.3. As shown in the figure, $1 / E$ and $1 / G$ are the intercept and the slope of the line, respectively. The line is called the "characteristic line" of the material, since the properties of the line are defined by the longitudinal and the through-thethickness shear moduli of the material. To establish the characteristic line of a material one needs at least two points in the coordinate system of Fig. 5.3. For this one must perform flexural tests (Fig. 5.1a) on two specimens having different $L / h$. Determining the slope of the load-deflection curve of each test provides a $F / \Delta$ value for Eqn (5.13), from which the corresponding $E^{\prime}$ is calculated. Since the $E^{\prime}$ value obtained from each test corresponds to a specific value of $J(h / L)^{2}$, the two tests on specimens with different $L h$ generates two 
distinct points in the coordinate system of Fig. 5.3 from which the characteristic line can be established. Knowing the characteristic line, one can easily determine the value of $E$ and $G$.

It is obvious that the accuracy and the reliability of the results obtained from the two tests are questionable. One can increase accuracy by conducting several tests, since each test generates a point in the coordinate system of the characteristic line. A linear regression analysis can then be used to establish the best fitted line. Statistically, the $E$ and $G$ values obtained from the intercept and the slope of the regression line are more reliable when the number of data points increases.

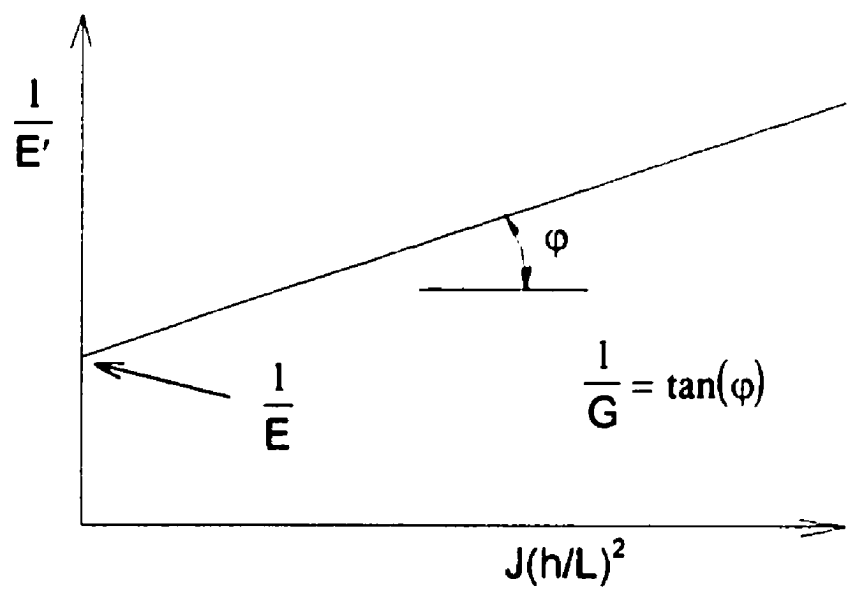

Figure 5.3 Schematic view of the characteristic line.

The value of $\bar{b}$ is also an important parameter influencing the value of $J$ and $E^{\prime}$. It is more convenient to write $\bar{b}$ as a fraction of the beam depth,

$$
\bar{b}=\alpha h
$$

in which case, Eqns (5.13) and (5.15) take the following forms, respectively

$$
E^{\prime}=\frac{F}{4 \Delta b}\left[\left(\frac{L}{h}\right)^{3}-2 \alpha^{2} \frac{L}{h}+\alpha^{3}\right]
$$




$$
J=1.2 \frac{\frac{L}{h}-1.5 \alpha}{\frac{L}{h}-2 \alpha^{2} \frac{h}{L}+\alpha^{3}\left(\frac{h}{L}\right)^{2}}
$$

The $J$ coefficient is a correction factor that includes the load and reaction distribution effects. For zero load distribution $(\alpha=0)$ the coefficient becomes 1.2 , however for $\alpha>0$, it is always smaller than 1.2. For beams with large $L / h$, the effect of the load distribution becomes insignificant, and as a result, the value of $J$ approaches 1.2. The influence of $L / h$ and $\alpha$ on $J$ is illustrated in Fig. 5.4.

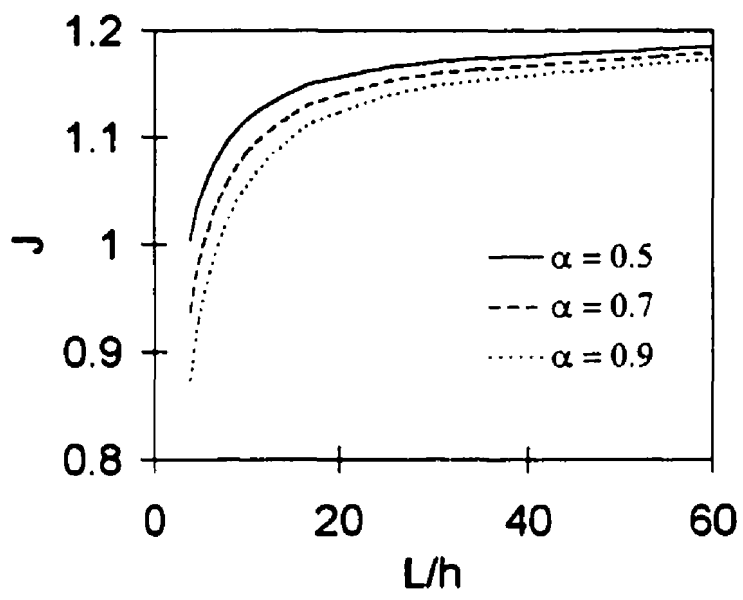

Figure 5.4 Variation of $J$ with respect to $L / h$ and $\alpha$.

When compared with the TTTI formulation, the application of the Timoshenko beam theory provides a much simpler and more practical procedure for evaluating the longitudinal and shear moduli from the VSM test data. As a result, the next two chapters of the thesis will focus on this procedure when investigating the integrity of the VSM and the reliability of the results obtained from the method.

\subsection{Summary and conclusions}

For simultaneous evaluation of the longitudinal and through-the-thickness shear moduli of FRPC by the VSM one needs a relationship between the applied load and the mid-span 
deflection that only depends on these two elastic moduli. For this purpose, the TTTI and Timoshenko beam theories were used in this chapter. While the relationship obtained from TTTI theory is believed to provide more accurate results, the application of the Timoshenko beam theory was shown to be simpler. The procedure for determining the said elastic moduli from the VSM results based on the two relationships were discussed separately.

When using the TTTI solution, one must have the result of tests on specimens with two different $L / h$. Determination of the elastic moduli requires one to perform a trial and error procedure, or to use the graphical solution provided in Fig. 5.2. Special statistical procedures will be needed if one wishes to determine the $E$ and $G$ values from testing specimens with more than two different $L / h$.

The solution based on the Timoshenko beam theory led to the introduction of a new terminology called the "characteristic line" of the material. One may conduct tests with different $L / h$ to produce several data points in the coordinate system used for constructing the characteristic line. The characteristic line is obtained by fitting the best line among the data points. The intercept and the slope of the characteristic line provide the reciprocal of the longitudinal and shear moduli of the material, respectively. This procedure is summarized in the flowchart of Fig. 5.5.

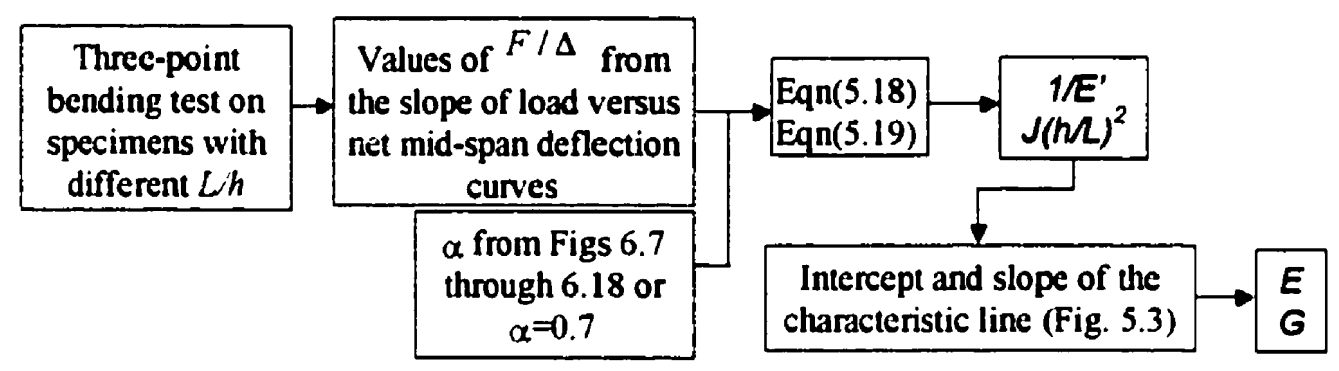

Figure 5.5 Flowchart for the VSM based on the Timoshenko beam theory. 


\section{Chapter 6}

\section{THEORETICAL INVESTIGATIONS}

Two possible methods for interpreting the VSM data were introduced in Chapter 5. The methods were based on two-dimensional models along with some simplifying assumptions. Therefore, to assess the efficiency and the reliability of the method, comprehensive theoretical and experimental investigations are needed. While the experimental investigations will be presented in the next chapter, the theoretical investigations are presented herein. Between the two methods of data processing developed in Chapter 5 , the method based on the Timoshenko beam theory is simpler and more practical. As a result, the attention will be focused on this method. The effect of several influential parameters on the accuracy and efficiency of the VSM are investigated and discussed below.

\subsection{Efficiency}

The efficiency of the VSM method depends on the variation of the shear effect on the response of FRPC when specimens with various $L / h$ are subjected to three-point bending. This variation is reflected in the magnitude of $1 / E^{\prime}$. Referring to Fig. 5.3, the variation of $1 / E^{\prime}$ determines the slope and the intercept value of the characteristic line from which the shear and the longitudinal moduli are obtained. Since there is always the possibility of obtaining different properties from one specimen to another, a wide variation of $1 / E^{\prime}$ in a set of tests is needed to compensate for possible variability due to errors in tests and material nonuniformity.

In order to investigate the variation of the shear effect in the response of the FRPC specimens, we multiply the two sides of Eqn (5.16) by $E$ to reach the following equation.

$$
\frac{E}{E^{\prime}}=1+J \frac{E}{G}\left(\frac{h}{L}\right)^{2}
$$


As the equation shows, the change in the shear effect is proportional to the change in $(h / L)^{2} E / G$ from one specimen to another. The characteristic of the equation for $\alpha=0.7$ is illustrated in Fig. 6.1. As the figure shows, for specimens made of a material with $E / G=30$, one can generate $47 \%$ change in the shear effect by changing $L / h$ from 8 to 30 . To get the same change for a material with $E / G=10, L / h$ has to vary from 4.5 to 30 . However, for a material with $E / G=2$, a wide change in $L / h$ from 4 to 30 , produces only a $9 \%$ change in the shear effect. It is, therefore, clear that for the proposed method to work effectively, one should be able to test specimens with relatively small spans. This factor is more critical when materials with smail $E / G$ are tested.

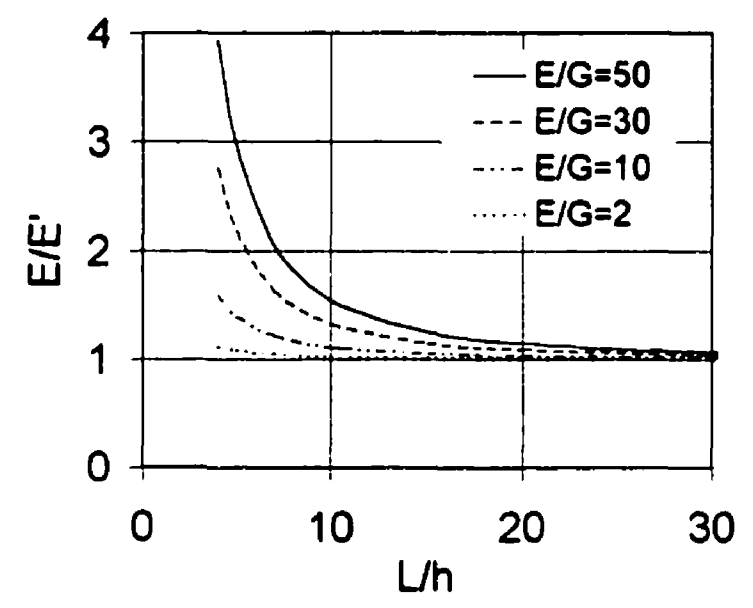

Figure 6.1 Variation of the shear effect in three-point bending.

\subsection{Effect of support friction}

When a specimen is subjected to three-point bending, the points over the supports move outward. This is due to the fact that the bottom surface of the specimen is under tension. Free movement occurs only if the interface friction between the specimen and supports is null. Otherwise, as shown in Fig. 6.2, two horizontal forces at the points of contact with the supports will act on the specimen. These forces counteract the applied load and tend to reduce the mid-span deflection. As a result, the flexural stiffness obtained from the test 
will be overestimated. To assess the extent of the phenomenon, the elasticity solution of the problem is derived. The solution will then be used to investigate the phenomenon for specimens with different material properties and with different $L / h$.

The elasticity solution for a simply supported beam subjected to harmonic loads at the top and bottom surfaces was presented in Section 3.1. The applied loads were distributed normal to the surfaces, and as a result, the shear stresses on these two surfaces were set to zero by applying Eqn (3.11). To obtain the solution for our present case, we assume that the shear stress at the bottom of the beam is not zero. Therefore, the boundary conditions for the top and bottom of the beam are expressed as follows

$$
\begin{aligned}
& \tau_{x=}(h / 2)=0 \\
& \tau_{x=}(-h / 2)=\tau_{b}(x) \\
& \sigma_{: z}(h / 2)=q_{t}(x) \\
& \sigma_{: z}(-h / 2)=q_{b}(x)
\end{aligned}
$$

where $q_{t}(x)$ and $q_{b}(x)$ are the harmonic loads on the top and the bottom of the beam respectively defined by Eqn (3.2), and

$$
\tau_{b}(x)=Q_{n} \cos (p x)
$$

where $p$ is defined by Eqn (3.3).

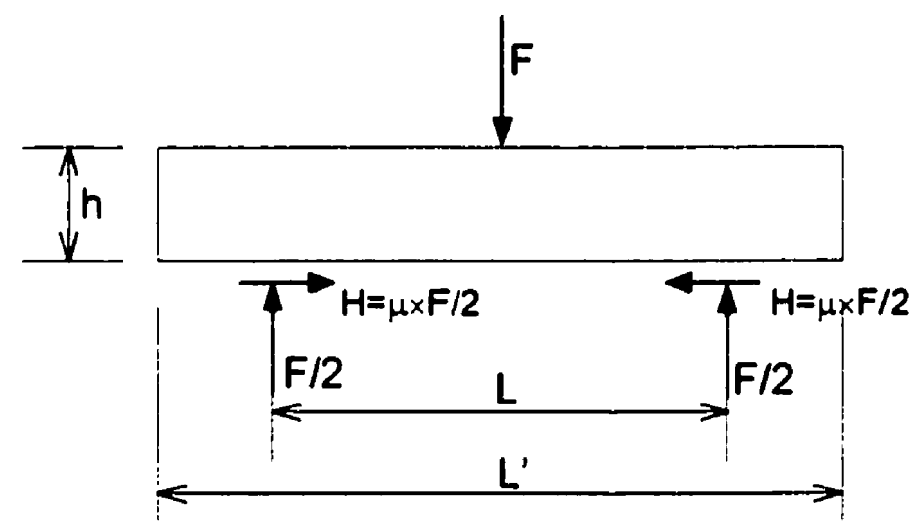

Figure 6.2 Free body diagram of beam under three-point bending when the supports are not friction-free. 
To include the effect of the horizontal reactions on the solution provided in Section 3.2 for a beam under three-point bending, one must transform the horizontal reactions to Fourier series as

$$
\tau=\sum_{n=1}^{\infty} Q_{n} \cos (p x) \quad n=1,3,5, \ldots
$$

where $\tau$ defines the distribution of the reactions on the bottom surface of the beam. To find the coefficients of the series one must multiply both sides of the equation by $\cos \frac{m \pi x}{L^{\prime}}$ and integrate over the entire length of the beam. Since for $n \neq m$ the result of $\int_{0}^{L} \cos \frac{n \pi x}{L^{\prime}} \cos \frac{m \pi x}{L^{\prime}} d x$ is zero, one obtains the following equation for the coefficients of Fourier series.

$$
Q_{n}=\frac{2}{L^{\prime}} \int_{0}^{L^{\prime}} \tau \cos \frac{n \pi x}{L^{\prime}} d x
$$

By changing the variable $x$ to

$$
x=\frac{L^{\prime}}{2}+\bar{y}
$$

one can write

$$
Q_{n}=(-1)^{\frac{n+1}{2}} \frac{2}{L^{\prime}} \int_{-L^{\prime} / 2}^{L^{\prime} / 2} \tau \sin (p \bar{y}) d \bar{y} \quad n=1,3,5, \ldots
$$

where, $\bar{y}$ is measured from the mid-span. Since both $\tau$ and $\sin (p \bar{y})$ are odd functions, the integrand in Eqn (6.8) is an even function. As a result, $Q_{n}$ can be determined by integrating only over half of the beam as

$$
Q_{n}=(-1)^{\frac{n+1}{2}} \frac{4}{L^{\prime}} \int_{0}^{L^{\prime} / 2} \tau \cos (p \bar{y}) d \bar{y} \quad n=1,3,5, \ldots
$$

Since the horizontal reactions are the result of friction, they can be calculated by the multiplication of the vertical reactions by a friction coefficient, $\mu$. Assuming that the 
horizontal reactions follow the same distribution as the vertical reactions (Eqn (3.17)), one can define the shear stress due to the friction, $\tau$, as

$$
\tau=-\frac{\pi \mu F}{8 \bar{c}} \cos \left(\frac{\pi \bar{x}}{2 \bar{c}}\right)
$$

where $\bar{x}$ is measured from the center of the support and the other variables have the same meaning as defined before. The negative sign occurs because a positive $F$ produces a negative shear stress. Substituting this equation into Eqn (6.9) and applying the following variable change

$$
\bar{y}=\frac{L}{2}+\bar{x}
$$

one reaches

$$
Q_{n}=(-1)^{\frac{n-1}{2}} \frac{\pi \mu F}{2 \bar{c} L^{\prime}} \int_{-\bar{c}}^{\bar{c}} \cos \frac{\pi \bar{x}}{2 \bar{c}} \sin \left(\frac{n \pi \bar{x}}{L^{\prime}}+\frac{n \pi L}{2 L^{\prime}}\right) d \bar{x} \quad n=1,3,5, \ldots
$$

After simplification, the above equation becomes

$$
Q_{n}=(-1)^{\frac{n-1}{2}} \frac{2 \mu F}{L^{\prime}} \frac{\cos (p \bar{c})}{1-4\left(\frac{p \bar{c}}{\pi}\right)^{2}} \sin \left(\frac{p L}{2}\right) \quad \text { for } \frac{p \bar{c}}{\pi} \neq \frac{1}{2}
$$

and

$$
Q_{n}=(-1)^{\frac{n-1}{2}} \frac{\pi \mu F}{2 L^{\prime}} \sin \left(\frac{p L}{2}\right) \text { for } \frac{p \bar{c}}{\pi}=\frac{1}{2}
$$

The computer program ELAS-3P introduced in Chapter 3 can also treat the above problem. The program accepts the friction coefficient as an input. When $\mu=0$, the horizontal reaction forces are zero, and as a result, the program simulates the problem as outlined in Chapter 3. For $\mu>0$, however, the effect of horizontal reactions are implemented duly.

To investigate the effect of the support friction, we consider two laminae with the following material properties 
Material I

$$
\begin{array}{ll}
E_{x x}=200 \mathrm{GPa} & E_{z=}=16.67 \mathrm{GPa} \\
G_{x z}=6.67 \mathrm{GPa} & \mu_{x=}=0.25
\end{array}
$$

Material II

$$
\begin{array}{ll}
E_{x x}=200 \mathrm{GPa} & E_{z=}=50 \mathrm{GPa} \\
G_{x=}=20 \mathrm{GPa} & \mu_{x z}=0.25
\end{array}
$$

which represent materials with $E_{x x} / G_{x z}=30$ and 10 , respectively, while $E_{z z} / G_{x e}$ for both of them is 2.5. The diameters of the loading nose and the support rollers are assumed to be the same and equal to $10 \mathrm{~mm}$. It is assumed that the beams are $2 \mathrm{~mm}$ thick and have $4 \mathrm{~mm}$ overhangs at both ends. The results of the calculations for $F=0.1 \mathrm{kN} / \mathrm{mm}$ are presented in Fig. 6.3. In this figure $\Delta$ and $\Delta_{0}$ are the net mid-span deflections with and without the effect of friction, respectively. As the figure shows, the effect of friction becomes more significant as $L / h$ becomes smaller. The effect of friction is also more significant for material II which has a smaller $E_{x x} / G_{x}$ value. These results can also be demonstrated by the application of the Timoshenko beam theory to the beam shown in Fig. 6.2. For this, one must add the mid-span deflection due to flexure, shear and the horizontal load. The total deflection can be expressed by

$$
\Delta=\frac{F}{b E}\left[\frac{1}{4}\left(\frac{L}{h}\right)^{3}+\frac{3}{10} \times \frac{E L}{G h}-\frac{3}{8} \mu\left(\frac{L}{h}\right)^{2}\right]
$$

where the last term in the brackets is the contribution of the horizontal reactions. The effect of friction as defined in Fig. 6.3 becomes

$$
1-\frac{\Delta}{\Delta_{0}}=\frac{1.5 \mu}{\frac{L}{h}+1.2 \frac{E}{G} \times \frac{h}{L}}
$$

Although Eqn (6.18) is approximate, it shows a similar trend to that shown in Fig. 6.3.

The above investigation shows that the support friction can have significant effect on the result of three-point bending. Moreover, the effect of friction changes with the changes in $L / h$. As a result, the friction effect will influence both the slope and the intercept of the 
characteristic line, thereby causing inaccuracy in the evaluated $E$ and $G$ values. Therefore, every effort should be made to minimize the amount of friction at the supports, with the clause that more precaution is needed when materials with small $E / G$ are tested.
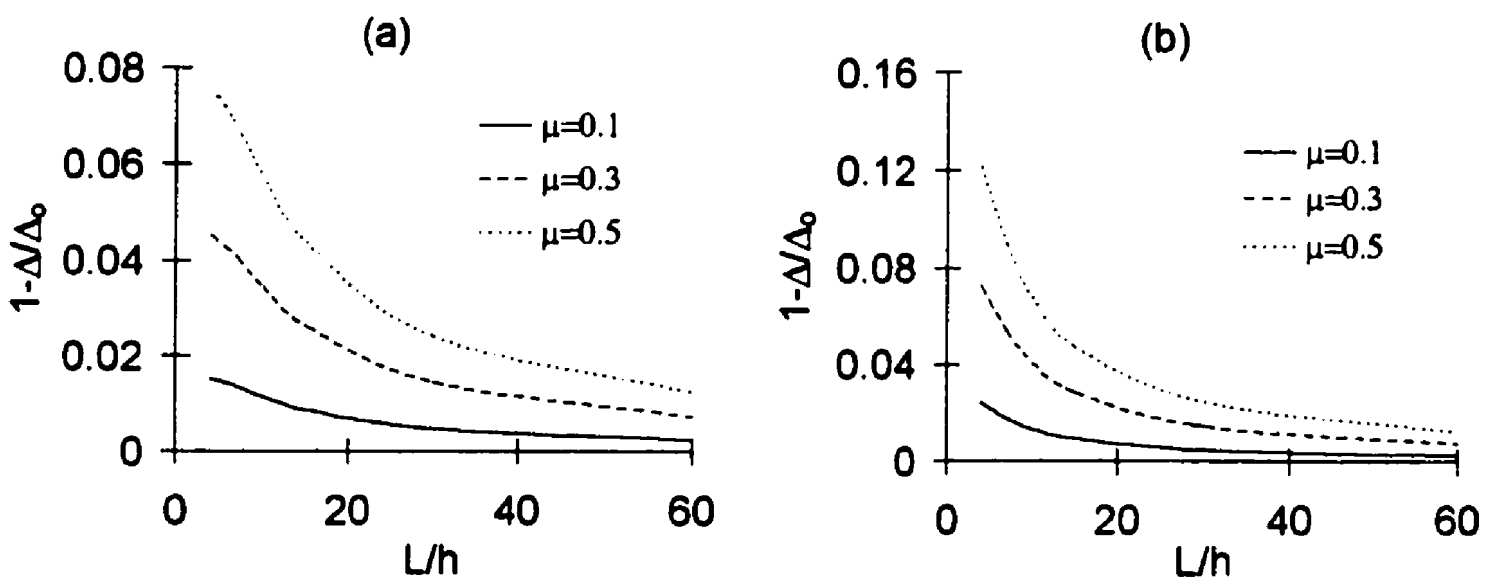

Figure 6.3 Effect of support friction on the net mid-span deflection. (a) For material type I. (b) For material type II.

\subsection{Specimen alignment}

As was discussed earlier, FRPC have three distinct values of $E$ and $G$ corresponding to the fiber direction and the two orthogonal axes to it. One can obtain these quantities by changing the alignment of the material axes (Fig. 1.1) with respect to the loading axes. Figure 6.4 shows two possible alignments for the specimens. In alignment type $\mathrm{A}$, the width of the specimen, $b$, in comparison to its other dimensions is small, and therefore, a state of plane stress governs. Furthermore, producing $L / h$ equal to 4 or even less is easily achievable. Consequently, not only the measurement of the moduli of materials with relatively small $E / G$ is possible, but also the accuracy and reliability of the results in general is high. The shortfall of this type of alignment is the difficulty in providing adequate lateral support to maintain the specimen in a vertical position and to prevent lateral buckling. With this alignment, one can determine either $E_{l l}$ and $G_{l:}$ or $E_{22}$ and $G_{l 2}$ by changing the direction of the material principal axes with respect to the loading axes. 
As it can be seen from Fig. 6.4, alignment type B is very simple and it enables one to obtain simultaneously either $E_{1 /}$ and $G_{13}$ or $E_{22}$ and $G_{23}$. Obviously, producing small $L / h$ in this case is not as easy as that in the alignment type A, unless relatively thick specimens are used. This problem may make the method less attractive in situations where $E / G$ ratio is small. Therefore, one may not find this alignment suitable for the evaluation of $E_{22}$ and G23.

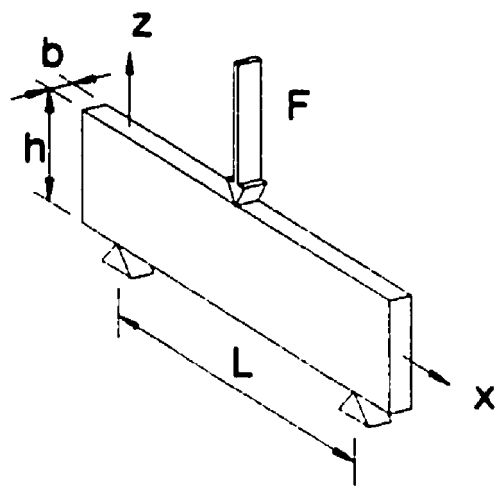

(A)

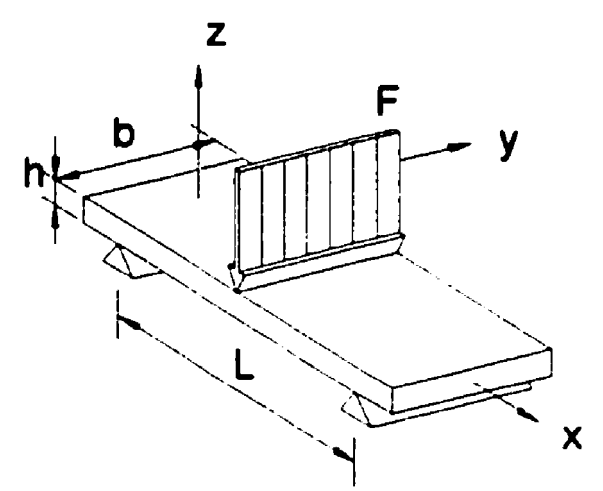

(B)

Figure 6.4 Two possible alignments for the VSM

It should be mentioned that the specimens in alignment type $B$ behave somewhere between the state of plane stress and plane strain. Having a constant width, specimens with large $L / h$ are almost in a state of plane stress, while as $L / h$ decreases, they approach the state of plane strain. From the theory of elasticity, it is known that the flexural stiffness of a plate under plane strain is $\left[1 /\left(1-v_{x y}{ }^{2}\right)\right]$ times larger than when the same plate is under plane stress state. Although the effect of this phenomenon will be considered in the establishment of $\alpha$ values in Section 6.5, it suffices to mention that the phenomenon can be problematic when materials with small $E / G$ and $v_{x y}$ are tested. For example, consider a material with $E / G=2.6$ and $v_{x y}=0.3$. For a specimen with $L / h=4$, the shear effect 
reduces the flexural stiffness by as much as $15 \%$ while, behaving under the state of plane strain increases the stiffness by $11 \%$. This leaves only a $4 \%$ difference for the determination of the slope of the characteristic line, which obviously will not provide sufficient accuracy and reliability. This problem, however, can be significantly reduced by selecting specimens with smaller width.

It is obvious that as with any new test method, the VSM must be subjected to a significant amount of theoretical and experimental investigations before any conclusion on the integrity of the method based on each possible alignment can be made. Thus, a comprehensive long term research program must be designed to investigate all the possible cases. Considering the present work as the starting point of this program, the attention will be focused only on one case. For this, alignment type B when the axis $l$ of the material is aligned in the $x$ direction will be considered.

\subsection{Influence of the interface contact and the non-linear behavior of the specimens}

The response of the test specimens subjected to three-point bending is a non-linear behavior. This non-linearity is due to the increase in the interface contact areas; that is the contact area between the specimen and the loading nose, and those between the specimen and the supporting rollers. This non-linearity becomes quite significant in specimens with small $L / h$. The problem was implicitly discussed in Section 3.3. However, to further illustrate the influence of this non-linear behavior on the net mid-span deflection, a critical case is investigated in here. For this, the exact elasticity solution presented in Section 3.2 is used. The properties of the beams are taken as follows

$$
\begin{array}{lll}
E_{x x}=200 G P a & E_{z=}=4 \quad G P a \\
G_{x=}=4 \quad G P a & U_{x=}=0.25
\end{array}
$$

which represent a highly anisotropic material. The geometry of the test is assumed to be $L^{\prime}=12 \mathrm{~mm}, L=8 \mathrm{~mm}, h=2 \mathrm{~mm}, r_{m}=5 \mathrm{~mm}$ and $r_{s}=3 \mathrm{~mm}$ (see Fig. 3.1). The beam is 
analyzed for a load $F$ varying from zero to a maximum of $F=400 \mathrm{~N}$ per millimeter width. The maximum load was taken from a test on a graphite/epoxy specimen with approximately the same geometry.

The result of the analysis is shown in Fig. 6.5a. The three different lines in the figure represent the total displacement, the semi-net displacement and the net displacement at mid-span. Referring to Fig. 3.1 and Eqn (3.30), these displacements are denoted by $\Delta_{T}$, $\Delta_{S N}, \Delta_{N}$. As Fig. 6.5a shows, the effect of non-linear behavior of the beam on the net midspan deflection is quite negligible. The initial slope of this curve is only $0.5 \%$ different from the slope of the best fit line through the first one quarter of the curve. The difference remains less than $2 \%$ when the slope of the best fit line is obtained by considering the full curve. On the other hand, the non-linearity of the total and semi-net displacements are quite significant. The slopes of these curves vary along their length and are different from the slope of net displacement. The initial slope of the semi-net and the total curves are $63 \%$ and $38 \%$ of the net curve, respectively. These values increase to $79 \%$ and $59 \%$ at the end of the curves, respectively.

Figure $6.5 \mathrm{~b}$ also shows the result of a flexural test on a specimen of graphite/epoxy with $L / h \equiv 5.5$. The solid line represents the measurement of the net mid-span deflection. The deviation of this line from the initial linear portion is due to the non-linear response of the material, and it does not reflect the geometric non-linearity due to the contact phenomenon. Owing to such material non-linearity, the slope of the load-deflection curve to be used in the evaluation of the elastic properties should be based on the slope of the linear portion at the beginning of the curve. Alternatively, one can use the initial tangent method to establish the slope. In either case, as the result of the numerical analysis in Fig. 3.5a shows, the effect of geometric non-linearity on the measured net mid-span loaddeflection curve is quite insignificant. 
(a)

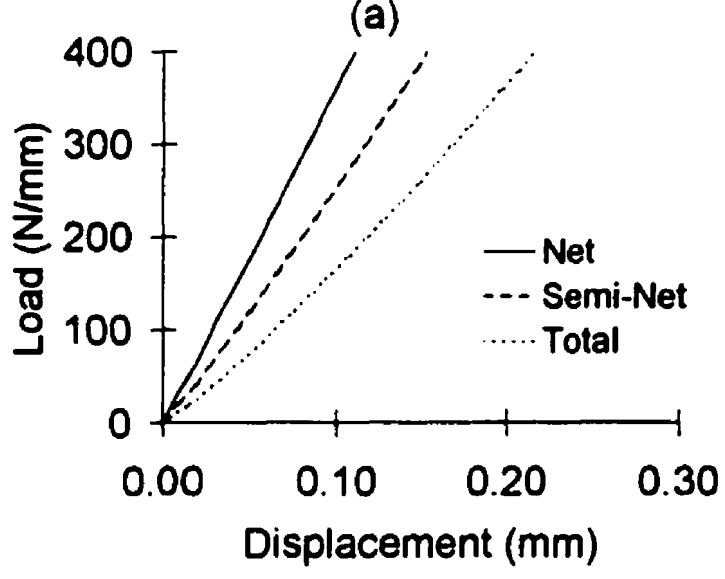

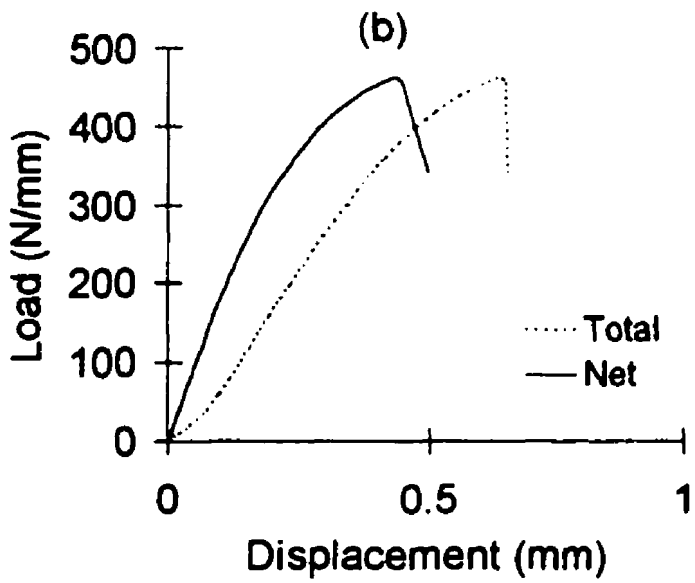

Figure 6.5 Load-displacement curves. (a) From the elasticity analysis. (b) From flexural test on a graphite/epoxy specimen with $\mathrm{L} / \mathrm{h} \cong 5.5$.

The effect of the diameters of the loading nose and supporting rollers were already investigated in Section 3.3. It was shown that the variation of the diameters had insignificant effect on the net mid-span deflection. In general, it is more desirable to use loading nose and supporting rollers with small diameters to make the test as close as possible to the assumptions of the theory. Furthermore, rollers with small diameters provide more freedom to produce small spans. However, the rollers and loading nose diameters must be kept large enough to avoid premature failure of the specimen due to stress concentration in the vicinity of the applied load and reactions.

\subsection{The value of " $\alpha$ "}

In Chapter 5 two different solutions were developed to determine the longitudinal and through-the-thickness shear moduli of materials from the VSM tests results. In both solutions, the applied load and the reactions were assumed to be distributed over a small length denoted by $2 \vec{b}$. This length, however, as defined by Eqn (5.17), was related to the depth of the beam by a coefficient called $\alpha$. The magnitude of $\alpha$ must be known before either solutions can be used for the VSM tests results. The value of $\alpha$ depends on the following factors. 
1- the alignment of the specimen with respect to the applied load ( Alignment type A or B in Fig. 6.4),

2- the solution for which it is being used,

3- the elastic properties of the material, and

4- the ratio of width to depth of the specimens $(b / h)$.

Considering the first two factors, it is obvious that there can be 4 different possibilities. The effect of the two last factors then must be investigated for each case separately. In the present work, attention will be focused on alignment type B. This will be in conjunction with the Timoshenko beam theory discussed in Section 5.2, which provides an easier and more straight forward solution compared to the TTTI theory. The evaluation of $\alpha$ and the other investigations will only be performed for $0^{\circ}$ specimens (where the axis 1 of the material is aligned in the $x$ direction).

\subsubsection{Evaluation of $\alpha$}

As was shown in Section 5.2, evaluation of the longitudinal and the shear moduli of FRPC requires one to determine the characteristic line in the coordinate system of Fig. 5.3. For this one must draw a straight line through the data points obtained from a series of threepoint bending tests performed on specimens with different $L h$. The evaluated moduli from this procedure, however, depends on the value of $\alpha$.

To establish the value of $\alpha$ for a particular set of material properties and $b / h$, the data points are produced from theoretical analysis using the same properties and $b / h$ in the analysis. The longitudinal and through-the-thickness shear moduli determined from the characteristic line should match the moduli used for the generation of the data points. If not, the value of $\alpha$ is changed in such a way until the two pairs of moduli are equal or very close to each other. The $\alpha$ value obtained in this way is called the "best $\alpha$ " for the specified case. 


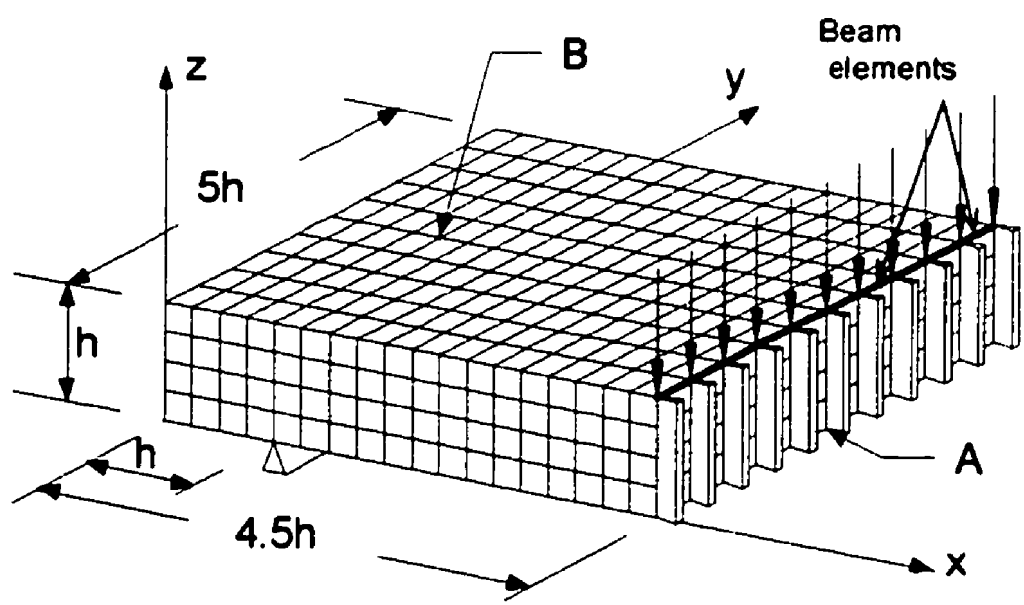

Figure 6.6 Finite element three dimensional model.

For the generation of the data points, one can follow the elasticity solution given in Chapter 3.2. However, the solution is only valid when a state of plane stress exists. For other situations, one should obtain the required data from a three dimensional finite element analysis. Here, the required data is obtained by conducting a set of finite element analyses. Three dimensional solid elements with 20 nodes (parabolic elements) are used to model beams with $L h=5,7,10,20$ and 30 and $b / h=1,3$ and 5. Figure 6.6 shows the finite element model used for $L / h=7$ and $b / h=5$. Because of symmetry, only half of the beam is modeled and, therefore, the displacements of the nodes in the $\boldsymbol{x}$ direction at midspan are constrained. Beam elements with very large flexural stiffnesses are also used to simulate the rigid loading nose. The vertical displacements of points $A$ and $B$, as shown in Fig. 6.6, are the major focus of the analyses. The net mid-span deflection is calculated from the vertical displacement of these points, and the flexural stiffness, $F / \Delta$, is determined subsequently.

The best $\alpha$ value for each case is calculated based on the results of three different values of $L / h$. For this, two ranges are considered; beams with $L h=5,7$ and 20, representing 
tests on short specimens, and beams with $L / h=7,10$ and 30 , simulating tests on longer specimens. The procedure was performed for 120 different sets of assumed properties, as tabulated in Appendix F. The different sets of the properties were taken in such a way that the effect of the following normalized quantities on $\alpha$ could be investigated. The values of the normalized quantities used in the investigation are as follows

$$
\begin{gathered}
\frac{E_{11}}{G_{13}}=10,20,30,40,50 \\
\frac{E_{33}}{G_{13}}=1,2,3,4 \\
\frac{G_{13}}{G_{23}}=1,2 \\
\cup_{12}=025,0.3,0.35
\end{gathered}
$$

It was also assumed that $E_{22}=E_{33}, G_{12}=G_{13}$ and $v_{13}=v_{23}=v_{12}$. Considering 3 different cases for $b / h$ and 5 different cases for $h / L$, a total of 1800 analyses were performed. For this, the general purpose finite element program NISA was used. The $\alpha$ values obtained from the finite element analyses and the procedure explained above are tabulated in Appendix G. These values are also presented in graphical form in Figs 6.7 through 6.18. As the plots show, $\alpha$ varies from 0.5 to 0.9 with an average value of 0.7 . 


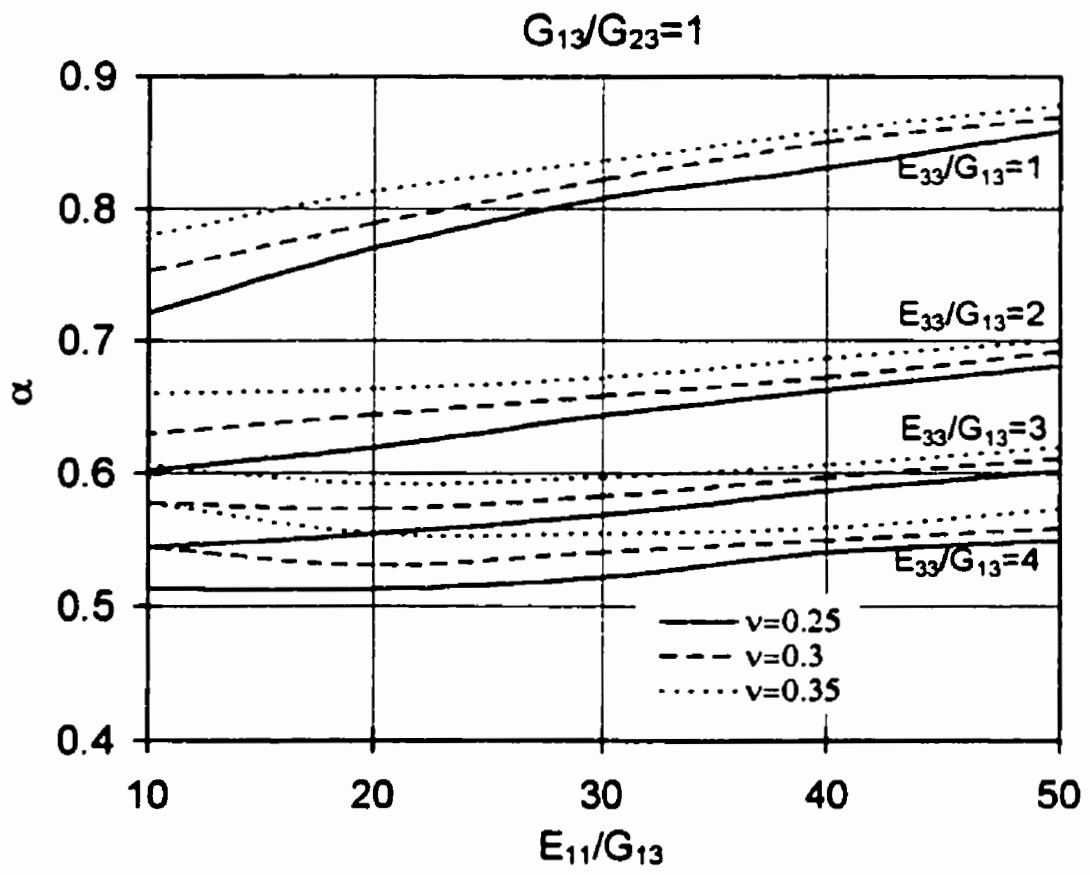

Figure 6.7 Variation of $\alpha$ for long specimens $(7 \leq L / h \leq 30)$ with $b / h=1$ and $G_{13} / G_{23}=1$.

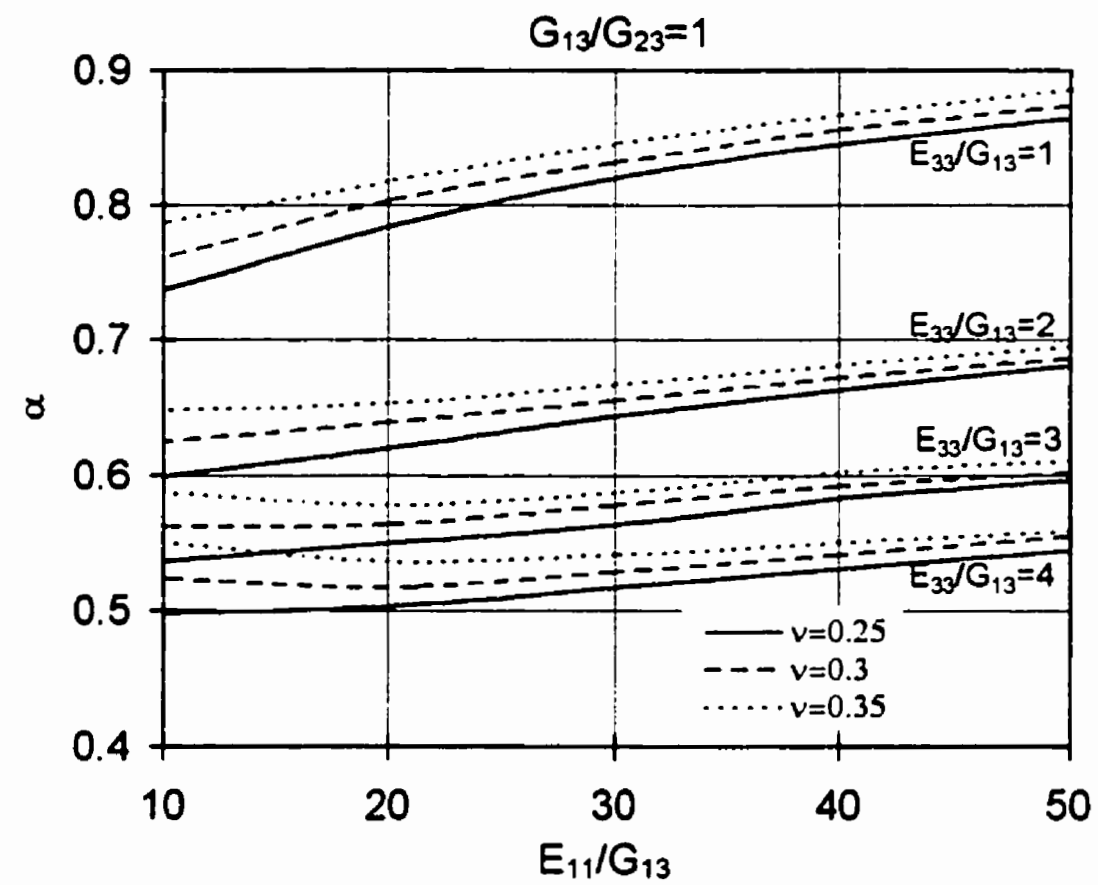

Figure 6.8 Variation of $\alpha$ for short specimens $(5 \leq L / h \leq 20)$ with $b / h=1$ and $G_{13} / G_{23}=1$. 


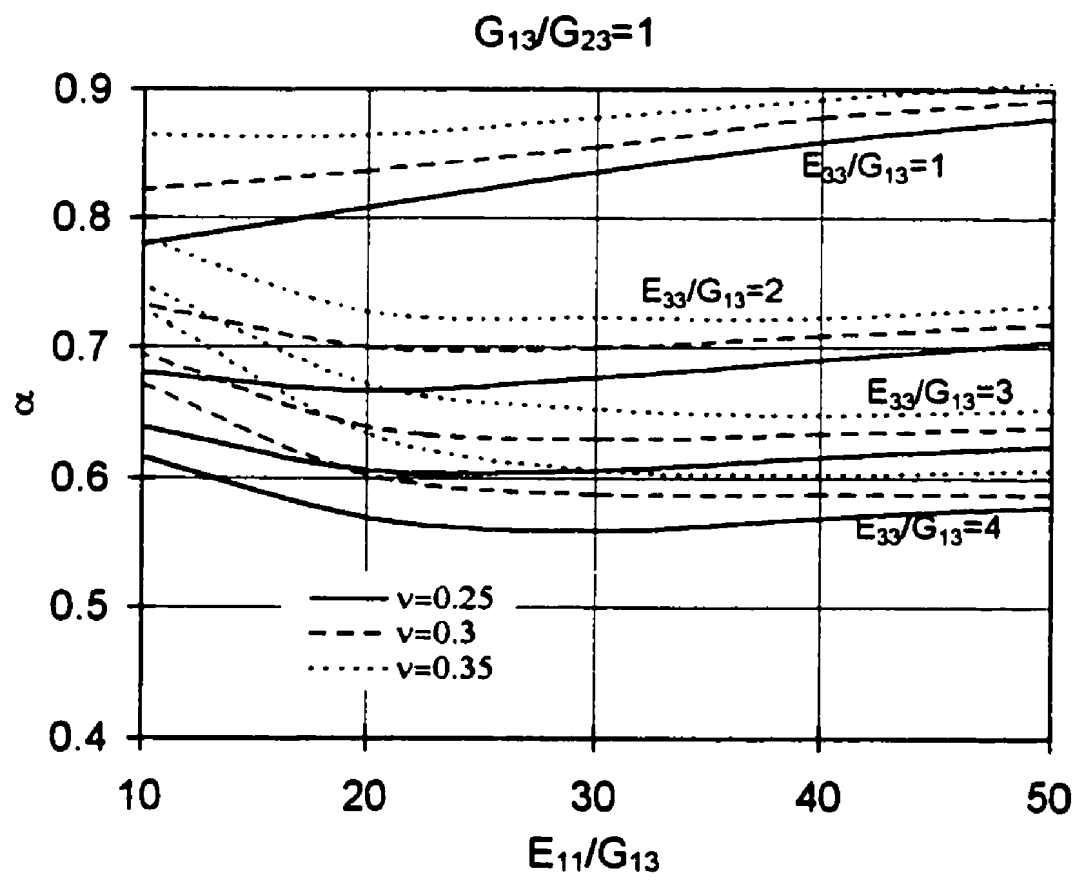

Figure 6.9 Variation of $\alpha$ for long specimens $(7 \leq L / h \leq 30)$ with $b / h=3$ and $G_{13} / G_{23}=1$.

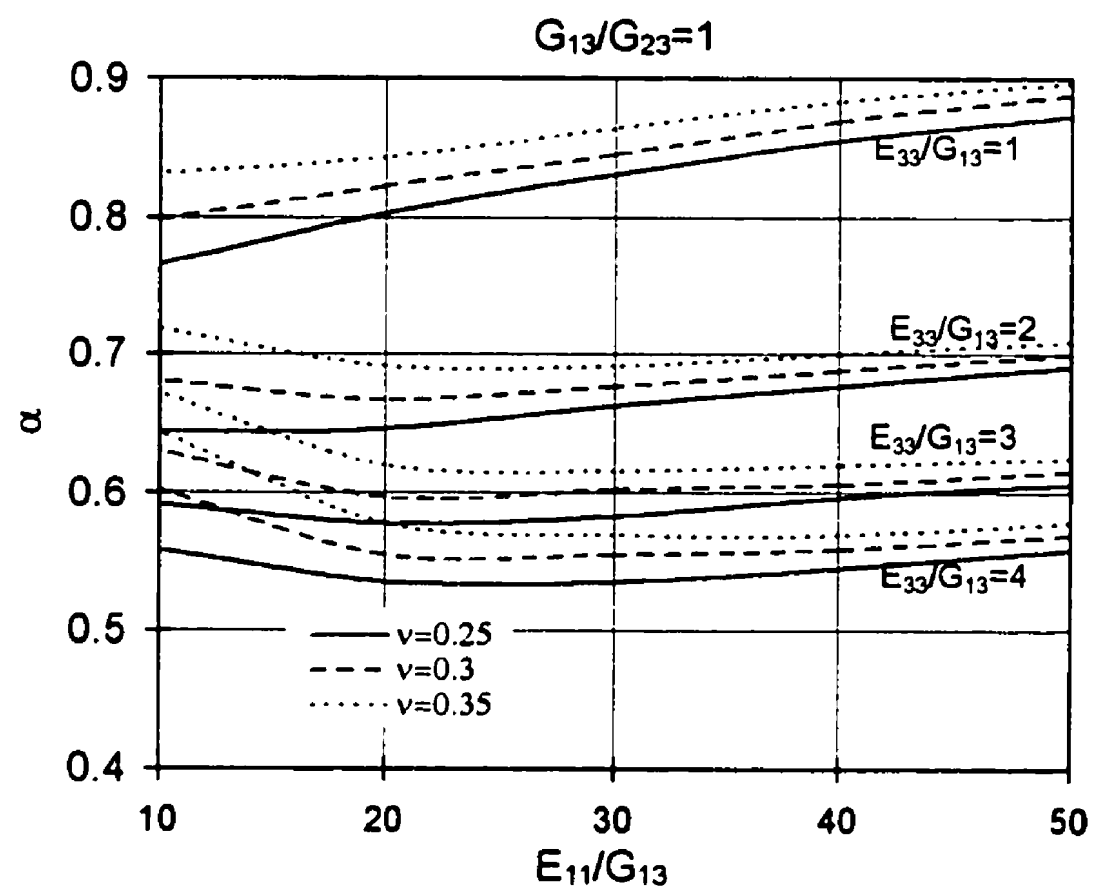

Figure 6.10 Variation of $\alpha$ for short specimens $(5 \leq L / h \leq 20)$ with $b / h=3$ and $G_{13} / G_{23}=1$. 


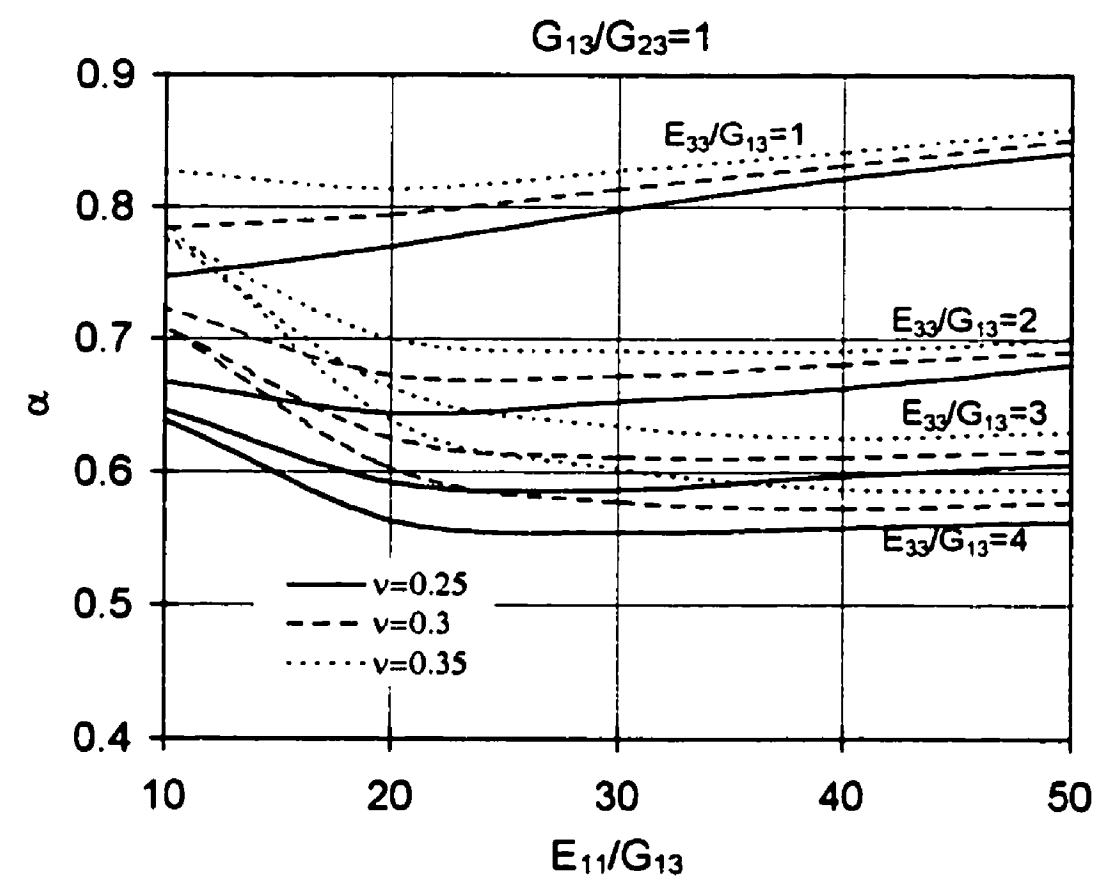

Figure 6.11 Variation of $\alpha$ for long specimens $(7 \leq L / h \leq 30)$ with $b / h=5$ and $G_{13} / G_{23}=1$.

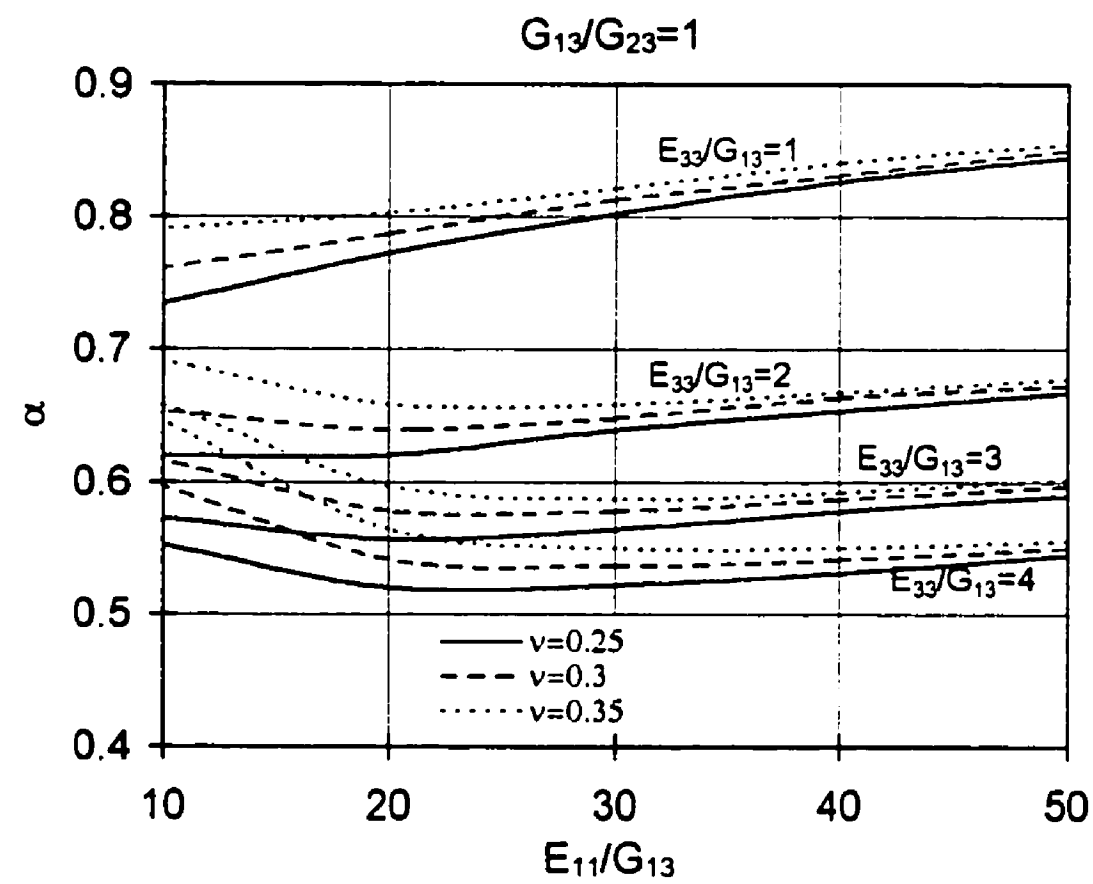

Figure 6.12 Variation of $\alpha$ for short specimens $(5 \leq L / h \leq 20)$ with $b / h=5$ and $G_{13} / G_{23}=1$. 


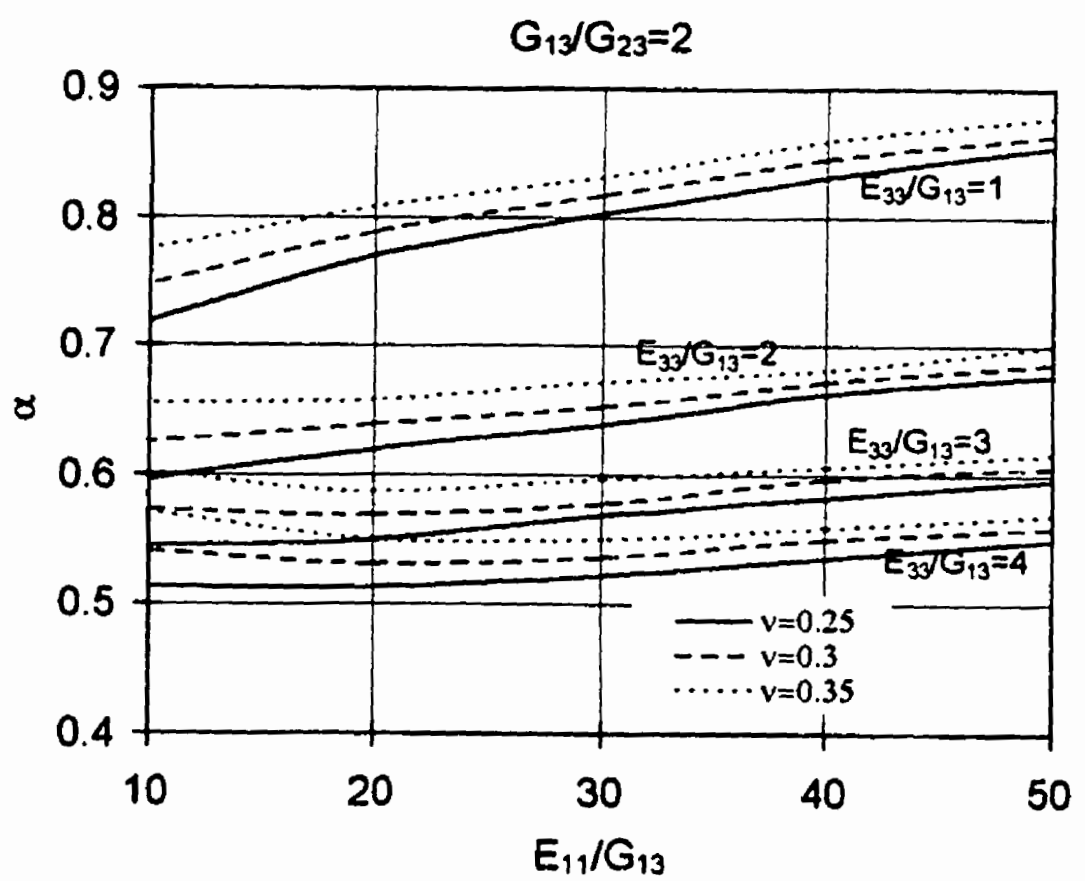

Figure 6.13 Variation of $\alpha$ for long specimens $(7 \leq L / h \leq 30)$ with $b / h=1$ and $G_{13} / G_{23}=2$.

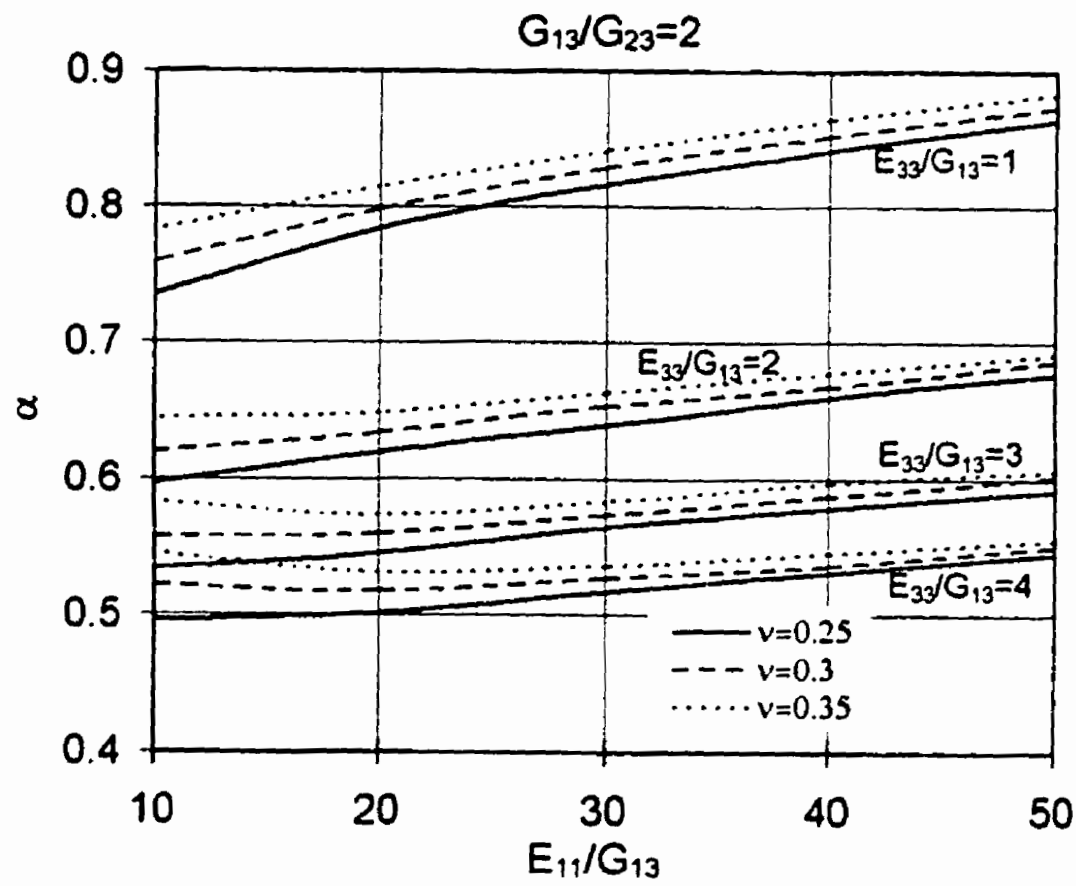

Figure 6.14 Variation of $\alpha$ for short specimens $(5 \leq L / h \leq 20)$ with $b / h=1$ and $G_{13} / G_{23}=2$. 


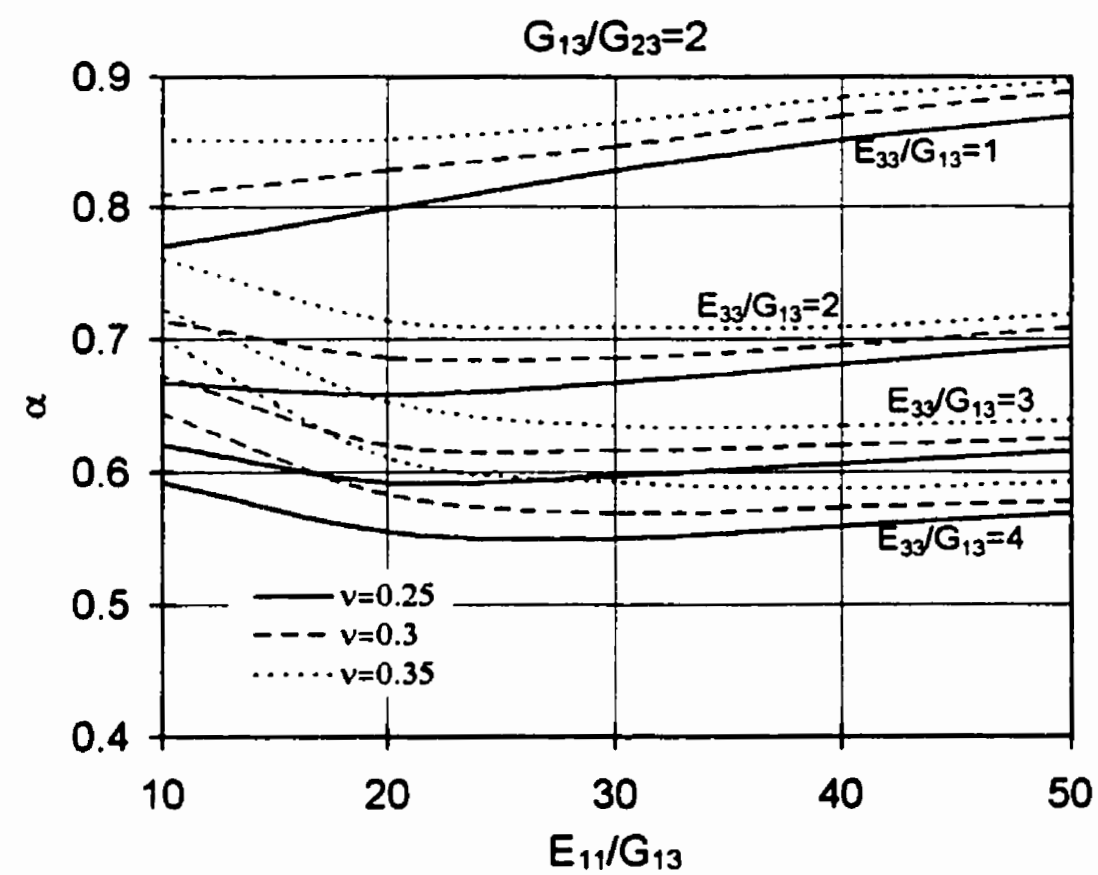

Figure 6.15 Variation of $\alpha$ for long specimens $(7 \leq L / h \leq 30)$ with $b / h=3$ and $G_{13} / G_{23}=2$.

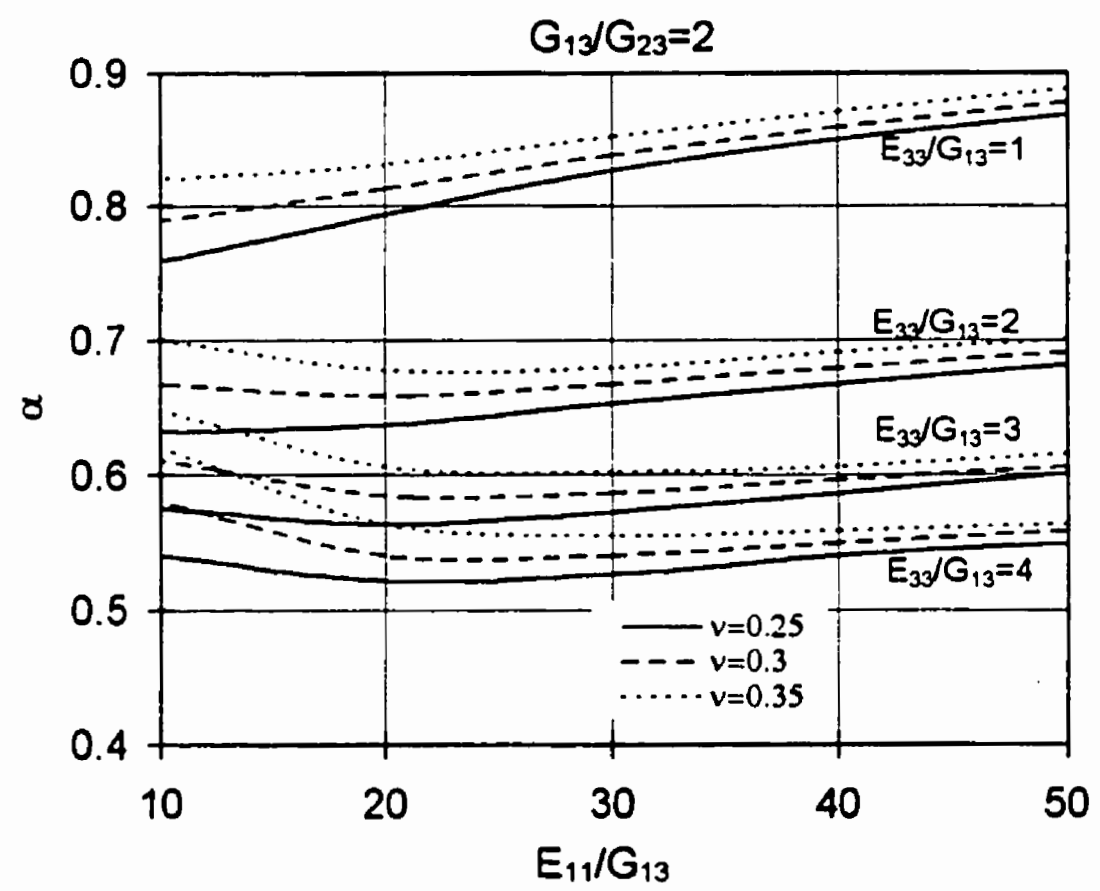

Figure 6.16 Variation of $\alpha$ for short specimens $(5 \leq L / h \leq 20)$ with $b / h=3$ and $G_{13} / G_{23}=2$. 


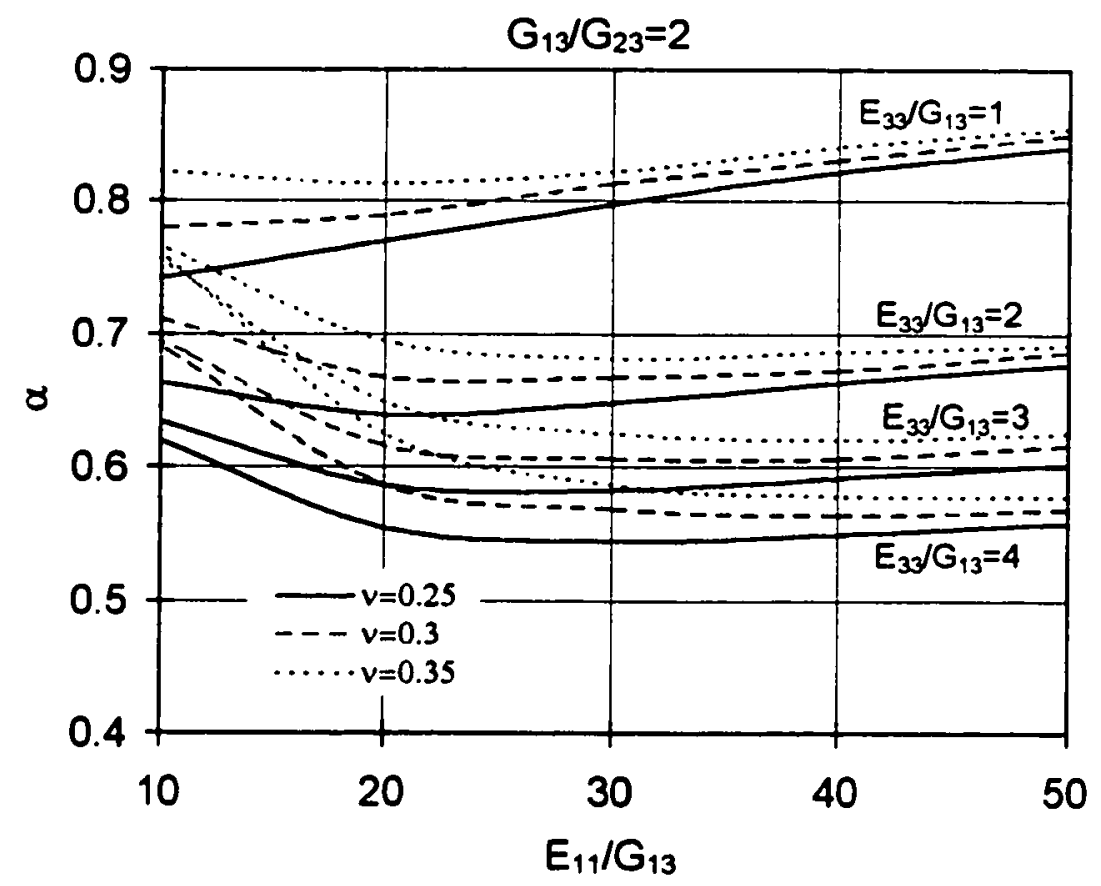

Figure 6.17 Variation of $\alpha$ for long specimens $(7 \leq L / h \leq 30)$ with $b / h=5$ and $G_{13} / G_{23}=2$.

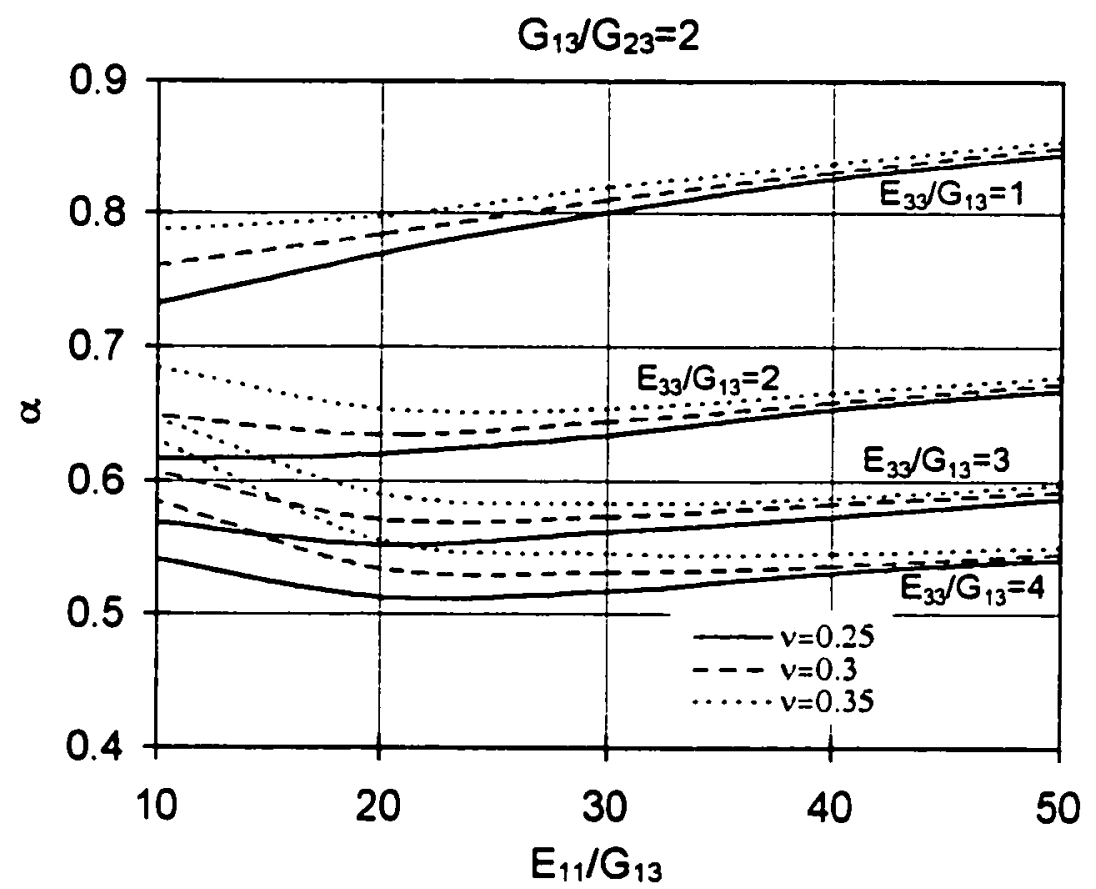

Figure 6.18 Variation of $\alpha$ for short specimens $(5 \leq L / h \leq 20)$ with $b / h=5$ and $G_{13} / G_{23}=2$. 


\subsubsection{The sensitivity of the method to the variation of $\alpha$}

The prerequisite for the use of Figs 6.7 through 6.18 is the knowledge of the material elastic properties. However, since these quantities cannot be accurately known before performing different types of tests, one must usually depend on previously available data or one's own judgment to attain some values for the normalized quantities needed in these Figures. As a result, the $\alpha$ value will be approximate. The question which arises is how sensitive the results of the VSM are to the variation in $\alpha$ (from its best value).

To answer this question, first the feasibility of taking the average value of $\alpha=0.7$ for all cases is investigated. For this, the values of $E$ and $G$ for the various cases used for the evaluation of the best $\alpha$ in Section 6.5.1 are calculated correspondingly. The difference between the correct $E$ and $G$ and the values obtained based on $\alpha=0.7$ indicates the sensitivity of the method to the variation of $\alpha$. The amount of errors in calculation of $E$ and $G$ for all cases are determined and are tabulated in Appendix $G$. These values show that the percentage error in no case exceeds $2 \%$ and $12 \%$, respectively, for $E$ and $G$, while the corresponding average errors are $0.58 \%$ and $4.4 \%$. The maximum error recorded for long specimens is lower than what was recorded for small specimens. Also cases with larger $b / h$ show less error for the value of $G$. For example, the maximum errors obtained for long specimens with $b / h=5$ are only $1 \%$ for $E$ and $5 \%$ for $G$. Recognizing the other uncertainties involved with the design of engineering structures and the variability involved with the manufacturing of the FRPC structural components, the error induced by taking the average value of $\alpha=0.7$ can be considered negligible. Moreover, the uncertainty of the results obtained from other established methods used for evaluating the longitudinal and shear moduli of composites are usually higher than $0.58 \%$ and $4.4 \%$, respectively. Therefore, one can conclude that using the average value of $\alpha=0.7$ is sufficient to obtain reasonably accurate $E$ and $G$ values for most practical purposes. 
The above investigation clearly indicates that the VSM is not sensitive to small variations in $\alpha$. While the average value of $\alpha=0.7$ provides sufficient accuracy, more accurate $\alpha$ can be easily obtained from Figs. 6.7 through 6.18 . For this, one needs to know the quantities of $E_{33} / G_{13}, E_{11} / G_{13}, G_{13} / G_{23}$ and $v$ of the material. However, a close examination reveals that $G_{13} / G_{23}$ has insignificant effect on the value of $\alpha$. The variation of the other quantities also do not change the value of $\alpha$ significantly. As a result, if no data exist for these quantities, approximate values obtained by the rule-of-mixture will provide sufficient accuracy. A more accurate value for $E_{11} / G_{13}$, however, can be obtained by using the $E_{11}$ and $G_{13}$ values determined based on the approximate value of $\alpha$ from the VSM.

The above investigations were conducted based on assuming a wide range of elastic properties to encompass all commonly used FRPC. Nevertheless, further validation was carried out by applying the above procedure to 8 commercially available FRPC. The elastic properties of these materials were obtained from Tsai (1988), and are listed in Table 6.1. The following assumptions were also made.

$$
\begin{aligned}
& E_{22}=E_{33} \\
& G_{12}=G_{13} \\
& G_{23}=\frac{2}{3} G_{12} \\
& u_{12}=v_{13}=v_{23}
\end{aligned}
$$

The best values of $\alpha$ and the errors associated with the evaluation of the $E$ and $G$ for long specimens with $b / h=5$ are tabulated in Table 6.2. The errors tabulated in the columns 3 and 4 are those resulting when the best values of $\alpha$ were used in the calculations; those resulting from $\alpha=0.7$ are also listed in the columns 5 and 6 . As can be seen from Table 6.2 , the changes in $\alpha$ has insignificant influence on the accuracy of $E$. Nevertheless, the corresponding error in no case exceeds $0.5 \%$. The error associated with the evaluation of the $G$ values based on $\alpha=0.7$, except for one case, is always less than $2 \%$. Figure 6.19 shows the characteristic lines of these materials based on $\alpha=0.7$. 
Table 6.1 The properties of the selected materials from Tsai (1988).

\begin{tabular}{ccccccc}
\hline $\begin{array}{c}\text { Materials } \\
\text { ID }\end{array}$ & Fibers & Resin & $E_{11}$ & $E_{22}$ & $G_{13}$ & $v_{12}$ \\
\hline l & T-300 & N5208 & 181 & 10.3 & 7.17 & 0.28 \\
2 & B(4) & N5505 & 204 & 18.5 & 5.59 & 0.23 \\
3 & AS & H3501 & 138 & 8.96 & 7.1 & 0.3 \\
4 & E-glass & Epoxy & 38.6 & 8.27 & 4.14 & 0.26 \\
5 & Kev 49 & PEEK & 76 & 5.5 & 2.3 & 0.34 \\
6 & AS 4 & Epoxy & 134 & 8.9 & 5.1 & 0.28 \\
7 & IM6 & PEEK & 203 & 11.2 & 8.4 & 0.32 \\
8 & T300 & Epoxy & 148 & 9.65 & 4.55 & 0.3 \\
\hline
\end{tabular}

Table 6.2 The variation of $\alpha$ and the associated percentage of error.

\begin{tabular}{|c|c|c|c|c|c|}
\hline \multirow[t]{2}{*}{$\begin{array}{l}\text { Materials } \\
\text { ID }\end{array}$} & \multirow[t]{2}{*}{$\begin{array}{c}\text { Best } \\
\alpha\end{array}$} & \multicolumn{2}{|c|}{$\begin{array}{l}\% \text { Error based } \\
\text { on the best } \alpha\end{array}$} & \multicolumn{2}{|c|}{$\begin{array}{c}\text { \%Error based on } \\
\alpha=0.7\end{array}$} \\
\hline & & $E$ & $G$ & $E$ & $G$ \\
\hline 1 & 0.719 & 0.22 & -0.06 & 0.20 & 0.63 \\
\hline 2 & 0.569 & 0.22 & 0.05 & 0.48 & -4.28 \\
\hline 3 & 0.742 & 0.26 & 0.08 & 0.22 & 1.77 \\
\hline 4 & 0.681 & 0.44 & -0.04 & 0.45 & -1.05 \\
\hline 5 & 0.653 & 0.39 & 0.04 & 0.48 & -1.56 \\
\hline 6 & 0.681 & 0.25 & 0.03 & 0.28 & -0.65 \\
\hline 7 & 0.747 & 0.28 & -0.04 & 0.22 & 1.72 \\
\hline 8 & 0.663 & 0.30 & -0.09 & 0.37 & -1.37 \\
\hline
\end{tabular}




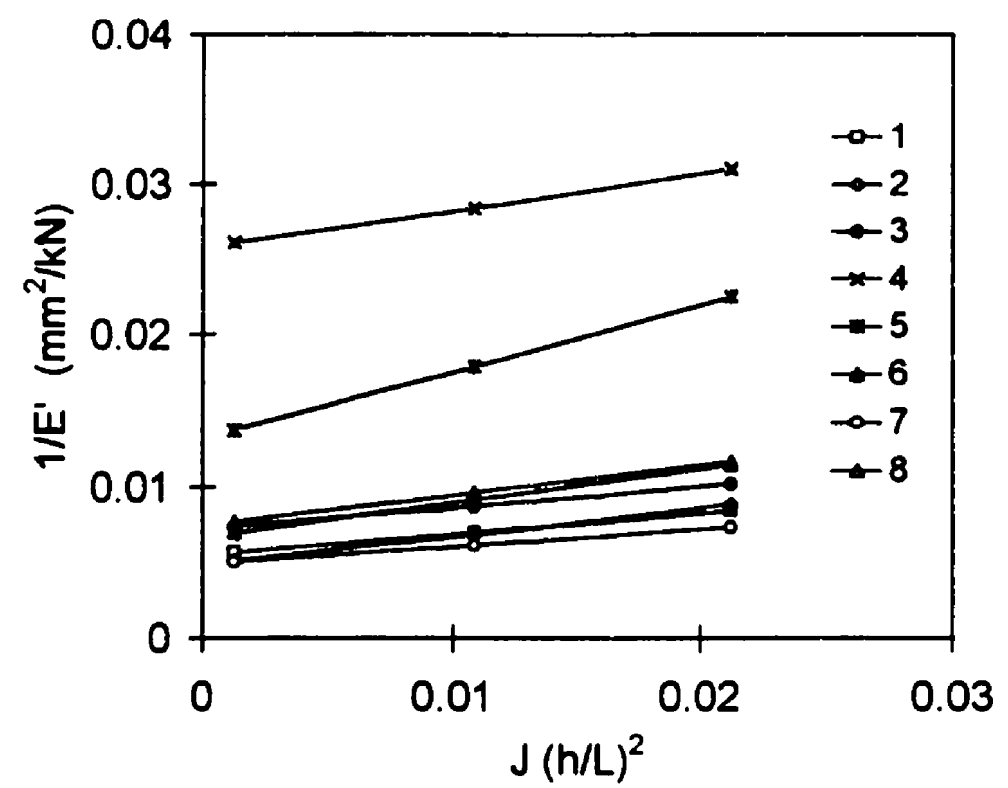

Figure 6.19 Characteristic lines of the materials identified in Table 6.1.

\subsection{Strain rate effect}

The standard procedure for a FRPC flexural test involves increasing the mid-span deflection at a constant rate while recording the corresponding load. The same procedure is adopted for the VSM. Since the mid-span deflection and the specimen strains are correlated, one can adjust the desired strain rate by changing the rate of mid-span deflection. To do this, the relations between the mid-span deflection rate and the strains rate must be known.

In determining the net mid-span deflection of a beam under three-point bending in Section 5.2 , the load and reactions were assumed to be distributed over a length equal to $2 \alpha h$. This was to eliminate differences between the Timoshenko beam theory and the exact elasticity solution. The assumption was important, since any inaccuracy in prediction of the mid-span deflection would directly affect the values of elastic moduli evaluated from the VSM tests results. However, for the strain rate, the situation is not so sensitive. As a result, and for the sake of simplicity, the load and the reactions are assumed to be 
concentrated (i.e. $\alpha=0$ ). Under this assumption, Eqns (5.10) and (5.11) are simplified as follows

$$
\begin{aligned}
\Delta_{\text {lexure }} & =\frac{F L^{3}}{4 E b h^{3}} \\
\Delta_{\text {shear }} & =0.3 \frac{F L}{G b h}
\end{aligned}
$$

respectively. The total deflection will then be determined by

$$
\Delta=\frac{F L^{3}}{4 E b h^{3}}+0.3 \frac{F L}{G b h}
$$

Taking the ratio of Eqns (6.22) and (6.23) gives

$$
\frac{\Delta_{\text {Fleare }}}{\Delta_{\text {Shear }}}=\frac{5 G L^{2}}{6 E h^{2}}
$$

On the other hand, the maximum longitudinal strain and the average shear strain are determined from the following equations

$$
\begin{aligned}
& \varepsilon_{\max }=\frac{3 F L}{2 E b h^{2}} \\
& \gamma_{\text {our }}=\frac{F}{2 G b h}
\end{aligned}
$$

Substituting Eqns (6.26) and (6.27) into Eqns (6.22) and (6.23), and dividing the two equations gives

$$
\frac{\Delta_{\text {Fleaure }}}{\Delta_{\text {Shear }}}=\frac{10 \varepsilon_{\max } L}{36 \gamma_{\text {arr }} h}
$$

The left hand sides of Eqns (6.28) and (6.25) are the same. As a result, one can equate the right hand sides of the two equations to obtain

$$
\frac{\varepsilon_{\mathrm{max}}}{\gamma_{\mathrm{arr}}}=3 \frac{G}{E} \times \frac{L}{h}
$$

or

$$
\frac{\dot{\varepsilon}_{\max }}{\dot{\gamma}_{\text {ar }}}=3 \frac{G}{E} \times \frac{L}{h}
$$

where $\dot{\varepsilon}_{\max }$ and $\dot{\gamma}_{a r}$ denote the longitudinal and shear strain rates of the specimen, 
respectively, in three-point bending. As Eqn (6.30) shows, the ratio of $\dot{\varepsilon}_{\max }$ to $\dot{\gamma}_{a r}$ is a function of $L / h$ of the specimen. Consequently, it is not possible to maintain a constant strain rate for both longitudinal and shear strains in the VSM from one specimen to another. For example by maintaining one of $\dot{\varepsilon}_{\max }$ and $\dot{\gamma}_{\text {aw }}$ constant for the specimens with $L / h$ varying from 4 to 30 , the other strain rate changes by an order of 7.5 . This phenomenon influences the accuracy of the VSM when strain rate sensitive materials are tested. Whether the influence is significant when characterizing FRPC, depends on the degree of strain rate sensitivity of a given material. The works of Daniel et al. (1982) and Adams and Adams (1990) provide good information for the strain sensitivity of some FRPC. Considering the range of strain rate change in the VSM and the strain sensitivity of FRPC, one can conclude that for most FRPC the variation of strain rate in the VSM does not have significant effect on the evaluated properties by this method. However a definite conclusion can be reached only when the results of VSM obtained under different strain rate arrangements are compared to each other. It is also important to compare the results obtained by the VSM with those obtained from other standard test methods. These comparisons will be carried out in Chapter 7. However, the theoretical background of the problem is presented here.

To obtain relationships between the strains and the mid-span deflection, Eqns (6.26) and (6.27) are substituted into Eqn (6.24). Taking the first derivative of the resulting equation with respect to time, one obtains

$$
\dot{\Delta}=\frac{1}{6} \dot{\varepsilon}_{\max } \frac{L^{2}}{h}+0.6 \dot{\gamma}_{a r} L
$$

where $\dot{\Delta}$ is the rate of the mid-span deflection. By combining this equation with Eqn (6.30), one can express the mid-span deflection rate of the beam in the following forms

$$
\dot{\Delta}=\dot{\varepsilon}_{\max } L\left[0.2 \frac{E}{G} \frac{h}{L}+\frac{1}{6} \frac{L}{h}\right]
$$




$$
\dot{\Delta}=\dot{\gamma}_{\text {avr }} L\left[0.6+0.5 \frac{G}{E}\left(\frac{L}{h}\right)^{2}\right]
$$

To maintain a constant longitudinal strain rate from one specimen to another, the rate of mid-span deflection must be determined from Eqn (6.32). Equation (6.33) is for maintaining a constant shear strain rate. Which equation to be used in practice depends on the sensitivity of $E$ and $G$ to strain rate, and to other parameters which will be discussed below. It is obvious that when one of the $E$ and $G$ values is considerably more sensitive to strain rate than the other, the corresponding strain rate must be kept constant. For other cases, one may find it useful to implement the following consideration to improve the consistency of the results.

The shear does not have significant influence on the result of the tests when specimens with $L / h>20$ are tested. However, the results are quite sensitive to the longitudinal properties. Therefore, in testing specimens with large $L / h$, maintaining a constant longitudinal strain rate has more importance than a constant shear strain rate. In specimens with $L / h<10$, when the longitudinal strain rate is kept constant, the shear strain rate varies by 2.5 when $L / h$ changes from 4 to 10 . Although this variation is quite insignificant, one may find it more useful to calculate the rate of the mid-span deflection by taking the average of the values obtained from Eqns (6.32) and (6.33). Nevertheless, for most common composite materials, the use of Eqn (6.32) for determining the mid-span deflection rate seems to be quite adequate.

A numerical investigation will better show the effect of strain rate sensitivity and the implementation of the above guidelines on the result of the VSM. For this, the sensitivity of $E$ and $G$ to strain rate are assumed to obey the following equations

$$
E=E_{s t}\left(1+0.05 \log \frac{\dot{\varepsilon}}{\dot{\varepsilon}_{s t}}\right)
$$




$$
G=G_{s t}\left(1+0.05 \log \frac{\dot{\gamma}}{\dot{\gamma}_{s t}}\right)
$$

where $\dot{\varepsilon}_{s t}$ and $\dot{\gamma}_{s t}$ are the standard rate for the evaluation of $E$ and $G$, respectively. These values usually are taken equal to $0.01 \mathrm{sec}^{-1} . E_{s t}$ and $G_{s t}$ are also the value of $E$ and $G$ at the standard strain rate. Considering the variation of $E$ and $G$ according to Eqns (6.34) and (6.35), Eqn (5.16) is used to produce data points in the coordinate system of Fig. 5.3. The characteristic line is drawn and the estimates of $E$ and $G$ are calculated accordingly. By comparison of these values with $E_{s t}$ and $G_{s t}$ used for the generation of the data points, the percentage error associated with the VSM is evaluated.

To carry out the investigation, three different cases of strain rate arrangements are considered. In case I, the mid-span deflection rate is assumed to be calculated from Eqn (6.32). As a result the shear strain rate is variable from specimen to specimen, while the longitudinal strain rate remains constant. The variation of $G$ is, therefore, determined from Eqns (6.35). In case II, Eqn (6.33) is used for mid-span deflection and the variation of $E$ is determined from Eqn (6.34). In case III, Eqn (6.32) is used for $L / h \geq 20$ and the average value of Eqns (6.32) and (6.33) is used for other $L / h$. The variation of $E$ and $G$ are accordingly determined from Eqns (6.34) and (6.35). The results of the investigation on five types of materials are presented in Table 6.3 and 6.4 for long and short specimens, respectively. The investigations for the long specimens were based on producing data points for $L / h=7,8,10,14$ and 30 , while $L / h=4,5,7,10$ and 20 were used for short specimens.

It is important to notice that the sensitivity to strain rate provided by Eqns (6.34) and (6.35) is considerably higher than the sensitivity of most FRPC. As a result, the errors tabulated in Tables 6.3 and 6.4 may be exaggerated for many cases. Nevertheless, in general, the results of the investigation confirm the earlier discussion on the application of Eqns (6.32) and (6.33) for the determination of the mid-span deflection rate. The results 
also show that when the mid-span deflection rate is determined properly, the effect of material strain rate sensitivity can be significantly reduced.

Table 6.3 Percent of error associated with different strain rate arrangements and the assumed sensitivity to strain rate for long specimens.

\begin{tabular}{|c|c|c|c|c|c|c|}
\hline \multirow[t]{3}{*}{$E / G$} & \multicolumn{6}{|c|}{ Percent of error } \\
\hline & \multicolumn{2}{|c|}{ Case I } & \multicolumn{2}{|c|}{ Case II } & \multicolumn{2}{|c|}{ Case III } \\
\hline & $E$ & $G$ & $E$ & $G$ & $E$ & $G$ \\
\hline 10 & 0.08 & 1.36 & 4.4 & 12.16 & 0.89 & 1.24 \\
\hline 20 & 0.16 & 0.15 & 2.89 & 6.65 & 0.40 & 0.55 \\
\hline 30 & 0.24 & 1.03 & 2.01 & 4.61 & 0.11 & 0.30 \\
\hline 40 & 0.31 & 1.65 & 1.38 & 3.54 & 0.09 & 0.12 \\
\hline 50 & 0.39 & 2.14 & 0.90 & 2.88 & 0.25 & 0.52 \\
\hline
\end{tabular}

Table 6.4 Percent of error associated with different strain rate arrangements and the assumed sensitivity to strain rate for short specimens.

\begin{tabular}{|c|c|c|c|c|c|c|}
\hline \multirow[t]{3}{*}{$E / G$} & \multicolumn{6}{|c|}{ Percent of error } \\
\hline & \multicolumn{2}{|c|}{ Case I } & \multicolumn{2}{|c|}{ Case II } & \multicolumn{2}{|c|}{ Case III } \\
\hline & $E$ & $G$ & $E$ & $G$ & $E$ & $G$ \\
\hline 10 & 0.22 & 0.16 & 3.34 & 5.16 & 0.72 & 0.79 \\
\hline 20 & 0.43 & 1.35 & 1.83 & 2.72 & 0.04 & 0.1 \\
\hline 30 & 0.63 & 2.23 & 0.95 & 1.86 & 0.37 & 0.85 \\
\hline 40 & 0.82 & 2.85 & 0.33 & 1.42 & 0.68 & 1.42 \\
\hline 50 & 1.02 & 3.34 & 0.16 & 1.15 & 0.95 & 1.88 \\
\hline
\end{tabular}

It is also important to note that both Eqns (6.32) and (6.33) are dependent on the value of $E / G$, a ratio that is not precisely known before testing. Therefore, one must use an approximate value for $E / G$. A close investigation of Eqns (6.32) and (6.33) shows that the rates calculated by these two equations are not very sensitive to the change in $E / G$. For example, for $L / h>20$ the variation of $E / G$ from 10 to 50 alters the rate calculated by Eqn (6.32) (the equation which is recommended for use for beams with large $L / h$ ) by less than $11 \%$. On the other hand, for $L / h<10$, when $E / G$ changes from 10 to 50 , the rates obtained by Eqns (6.32) and (6.33) vary less than 2.7 and 3.5 times, respectively. Recognizing these 
facts, assuming an average value of $E / G=30$ can be considered quite accurate for most practical purposes. However, one can easily estimate a more reasonable value for $E / G$, based on the rule-of-mixture, or from the literature.

\subsection{Efrect of lateral Friction}

For the evaluation of $\alpha$ in Section 6.5.1, the lateral ( $y$ direction) movements of the finite element models at the supports and loading nose were assumed to be free. However, in practice, due to the existence of the friction forces such an assumption may not be fully valid. The magnitude of this friction, even though it may be very small, is unknown. To examine the influence of the friction quantitatively, the finite element models used in section 6.5.1 (Fig. 6.6) are used to examine two extreme cases. In one case, the nodes on the contact lines are restrained against lateral displacement. This case simulates the extreme situation where the friction between the contacting surfaces in the y direction is very large. In the other case, the nodes are allowed to move freely laterally, thus simulating a friction free contact. The investigation is conducted for the material properties used in section 6.5.3 (Table 6.1). To determine the effect of friction for the worst condition, beams with $L / h=4$ and $b / h=5$ are examined. The ratio of the net midspan deflection obtained from the two models are determined and tabulated in Table 6.5. As the results show, the difference between the results of the two cases are quite insignificant, leading to the conclusion that friction between the contacting surfaces in the lateral direction is not an influential parameter.

Table 6.5 The ratio of the net mid-span deflection obtained from models with and without lateral friction at the supports.

\begin{tabular}{ccccccccc}
\hline Material ID & 1 & 2 & 3 & 4 & 5 & 6 & 7 & 8 \\
Ratio & 1.0043 & 1.0019 & 1.0046 & 1.0013 & 1.0037 & 1.0036 & 1.0054 & 1.0036 \\
\hline
\end{tabular}




\subsection{Summary and conclusions}

The effect of several influential factors on the efficiency and the accuracy of the VSM was theoretically investigated and discussed. Between the two possible alignments for testing the specimens (Fig. 6.4), the alignment type $B$ was the focus of the investigations. The effect of lateral friction at the supports was shown to be insignificant, while maintaining friction-free hinge supports was shown to be very important for obtaining accurate results. The non-linear behavior due to the contact of the specimen with the loading nose and the supporting rollers was shown to have insignificant effect on the net mid-span deflection. The values of $\alpha$ for materials with different properties were established. It was found that this quantity varied between 0.5 and 0.9 , while the use of the average value of $\alpha=0.7$ was shown to be sufficient for practical purposes. The influence of the variation of the longitudinal and shear strain rates from one specimen to another was discussed. Considering the relative insensitivity of most FRPC to strain rate, it was postulated that the range of changes in the strain rate in the VSM would not have significant effect on the results of the tests. Nevertheless, special guidelines and equations were provided to obtain the best results.

In general, the theoretical investigation validates the integrity of the VSM as a promising method for the simultaneous evaluation of the longitudinal and the through-the-thickness moduli of FRPC. The errors produced due to the nature of the method and those associated with the simplifying assumptions are all less than other uncertainties present in the design of FRPC engineering structures. In addition, the accuracy of the results are better than or at least comparable to the results of other available test methods. 


\section{Chapter 7}

\section{EXPERIMENTAL INVESTIGATIONS}

This chapter discusses the results of the experimental investigations designed to assess the integrity of the VSM. The materials considered in these investigations were unidirectional graphite/epoxy, unidirectional Kevlar/epoxy and unidirectional E-glass/epoxy. The investigations were to evaluate the following issues:

a) the applicability of the VSM for the selected materials,

b) comparison of the longitudinal and the shear moduli values determined by the VSM with other common test methods in practice,

c) the reliability of the VSM test results, and

d) the effect of the variable strain rate inherent in the VSM on the result of the test.

The applicability of the VSM method was assessed by the scatter of the data points obtained from the test results when they were plotted in $1 / E^{\prime}$ versus $J(h / L)^{2}$ coordinates system. A linear distribution of the data points was interpreted to mean that the VSM was a viable test method to the selected material.

The reference test method for the comparison of the longitudinal modulus evaluated by the VSM for all cases was the ASTM D3039-93 tensile test. However, as was mentioned in Chapter 2, the evaluation of the through-the-thickness shear modulus $\left(G_{13}\right)$ of the materials is only possible by the losipescu shear test (ASTM D5379-93) which requires a $20 \mathrm{~mm}$ thickness specimen; a configuration usually not easy to prepare. As a result, depending on the situation, one or two of the common test methods for the in-plane shear properties, such as the $\pm 45^{\circ}$ (ASTM D3518-94), the Iosipescu (ASTM D5379-93) and the $10^{\circ}$ off-axis shear tests, were used to assess the shear modulus obtained by the VSM. It should be mentioned that theoretically, if the distribution of fibers through the thickness and through the width of the laminate follows the same pattern, $G_{13}$ and $G_{12}$ should be 
equal. In practice, however, the situation may not be ideal. For instance, in hand lay-up laminates, the layer interfaces are more susceptible to defects and therefore a smaller value for $G_{13}$ can be expected.

The reliability of the VSM was estimated by determining the $95 \%$ confidence interval for the test results. This was accomplished by a linear regression analysis for the material characteristic line.

To assess the effect of variable strain rate from one specimen to another, different sets of specimens were prepared from each material. The test speed for each set was determined in such a manner that either the longitudinal or the shear strain rate remained constant for all the specimens in the given set. Equations (6.32) and (6.33) were used for this purpose. Since a constant net mid-span deflection rate could not be maintained through the loading device, the values from Eqn (6.32) and (6.33) were used directly to establish the speed of the actuator of the loading device.

A special apparatus was designed and fabricated for the VSM. It was to allow the measurement of the net mid-span deflection and also to allow testing of specimens with small spans. The schematic and the description of the apparatus are documented in Appendix $\mathrm{H}$. The loading device was an universal MTS testing machine.

\subsection{Investigation on graphite/epoxy}

Experimental investigation on graphite/epoxy was carried out by conducting the VSM, the ASTM D3039-93 tensile test, the ASTM D3518-94 \pm 45 shear test and the ASTM D537993 Iosipescu shear tests. The specimens were cut from $300 \times 300 \mathrm{~mm}$ hand lay-up panels made of 24 layers of Fiberite graphite/epoxy prepreg. The panels were vacuum bagged and were cured in an autoclave using the curing cycle specified by the supplier of the prepregs. The specimens for the VSM, the tensile and the Iosipescu shear tests were cut 
from unidirectional panels, while a symmetric cross-ply laminate was used to provide the specimens for the $\pm 45^{\circ}$ shear tests. The panels were cut into strips of approximately equal width. These strips were then used to prepare specimens with different lengths. To eliminate the effect of resin reach layers on two sides of the specimens and also to ensure smooth surfaces and uniform thickness, the specimens were sanded with No. 400 silicon carbide powder. The final thickness of the specimens was between 2.51 and $2.72 \mathrm{~mm}$ for the VSM tests, between 2.33 and $2.51 \mathrm{~mm}$ for the tensile tests, between 2.41 and $2.51 \mathrm{~mm}$ for the \pm 45 shear tests and between 2.55 and $2.58 \mathrm{~mm}$ for the Iosipescu shear tests. The width of the specimens were about $12.5,13$ and $25 \mathrm{~mm}$ for the VSM, tensile and for $\pm 45^{\circ}$ shear tests, respectively. The dimensions of specimens for the Iosipescu shear test were based on the dimensions specified by the ASTM D5379-93. The details for the specimens are presented in Appendix I.

Four sets of specimens were prepared for the VSM. The speed of the test for the first two sets were determined by assuming a constant longitudinal strain rate of $\dot{\varepsilon}_{\max }=0.01 \mathrm{sec}^{-1}$ and assuming $E / G=30$ for use in Eqn (6.32). For the third set, the value of $E / G$ in Eqn (6.32) was assumed to be equal to 10 , while the longitudinal strain rate was assumed to be the same as that used for the first two sets. The fourth set of specimens were subjected to a constant shear strain rate. Equation (6.33) with the assumption of $\dot{\gamma}_{a r r}=0.01 \mathrm{sec}^{-1}$ and $E / G=30$ was used for this set. Table 7.1 summaries the different strain rate cases.

Table 7.1 Specifications of strain rate cases for different sets of graphite/epoxy specimens.

\begin{tabular}{ccccc}
\hline Designation & Equation & $E / G$ & $\dot{\varepsilon}_{\max }$ & $\dot{\gamma}_{\text {ar }}$ \\
\hline GR-1 & 6.32 & 30 & 0.01 & var. \\
GR-2 & 6.32 & 30 & 0.01 & var. \\
GR-3 & 6.32 & 10 & 0.01 & var. \\
GR-4 & 6.33 & 30 & var. & 0.01 \\
\hline
\end{tabular}


Each set of specimens were tested with 5 different spans, for $L / h$ from 5.52 to 30.05 . For each span, at least three specimens were tested. Each specimen was tested twice. In the first trial, depending on the linearity limit, the specimen was loaded to about $50 \%$ to $80 \%$ of the failure load. The test was stopped before the specimen showed significant nonlinearity. In the second trial, the specimen was loaded until failure. The initial slope of the load-deflection curve of each test was evaluated. This value was used for $F / \Delta$ in Eqn (5.18) to calculate the corresponding $E^{\prime}$. The plots of $1 / E^{\prime}$ versus $J(h / L)^{2}$ for the results obtained from the tests for each set, and the corresponding materials characteristic lines are presented in Figs 7.1 through 7.4. The $E$ and $G$ values, respectively, determined from the reciprocal of the intercept and the slope of the characteristic lines for different sets of the specimens are tabulated in Table 7.2. The approximate value of $\alpha=0.7$ was used for all cases. The load-displacement curves for all these specimens are included in Appendix J.

The plot of the test data in Figs 7.1 through 7.4 for both loadings follow a clear linear trend which confirms the applicability of the proposed method for the selected material. The $E$ and $G$ values obtained for different strain rate cases (Table 7.2) are quite similar, their differences being in the acceptable range of accuracy in practice. Furthermore, as Fig. 7.5 shows an obvious pattern between the results obtained for different strain rate cases (Table 7.1) does not exist. Consequently, it can be concluded that the variable strain rate, inherent in the VSM, is not problematic for graphite/epoxy. 

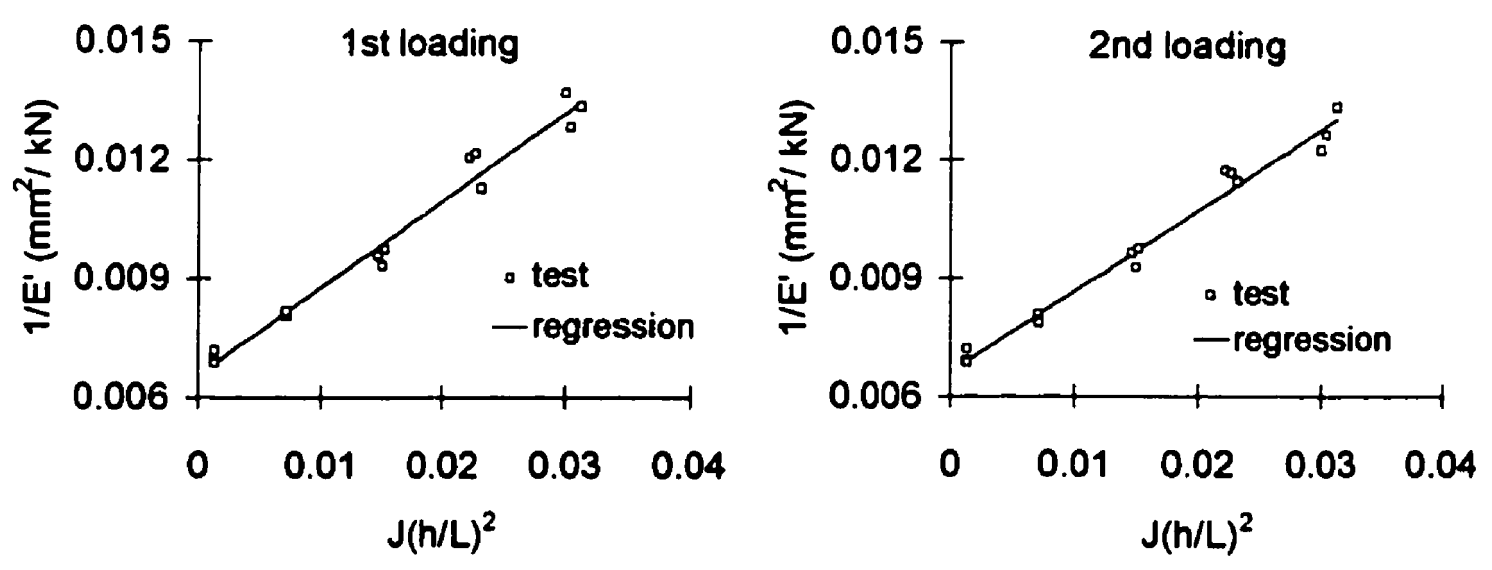

Figure 7.1 Plot of $1 / E^{\prime}$ versus $J(h / L)^{2}$ and the material characteristic line for GR-1 graphite/epoxy specimens.
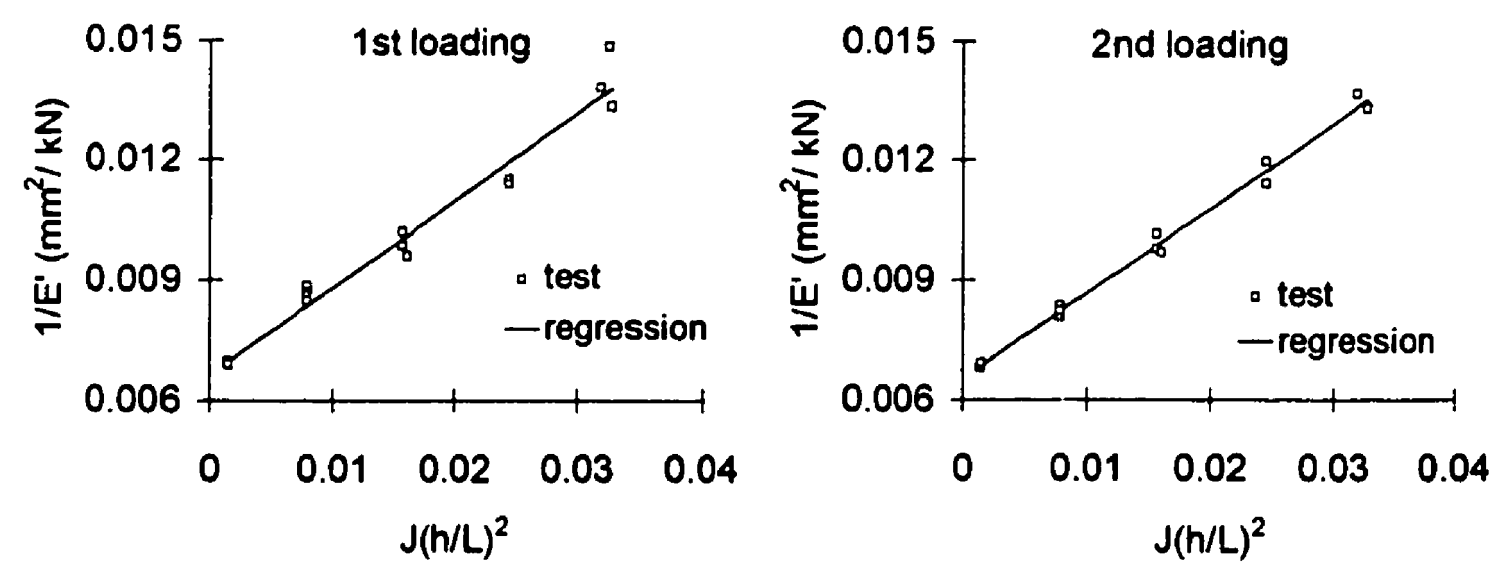

Figure 7.2 Plot of $1 / E^{\prime}$ versus $J(h / L)^{2}$ and the material characteristic line for GR-2 graphite/epoxy specimens. 

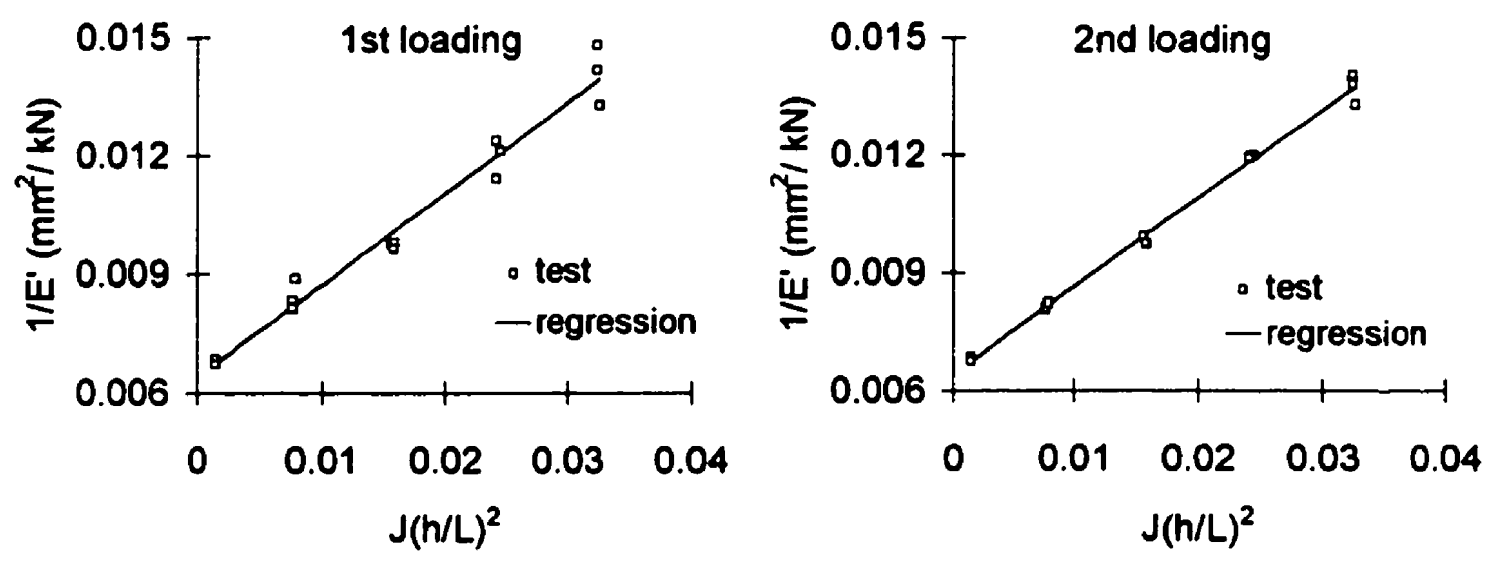

Figure 7.3 Plot of $1 / E^{\prime}$ versus $J(h / L)^{2}$ and the material characteristic line for GR-3 graphite/epoxy specimens.
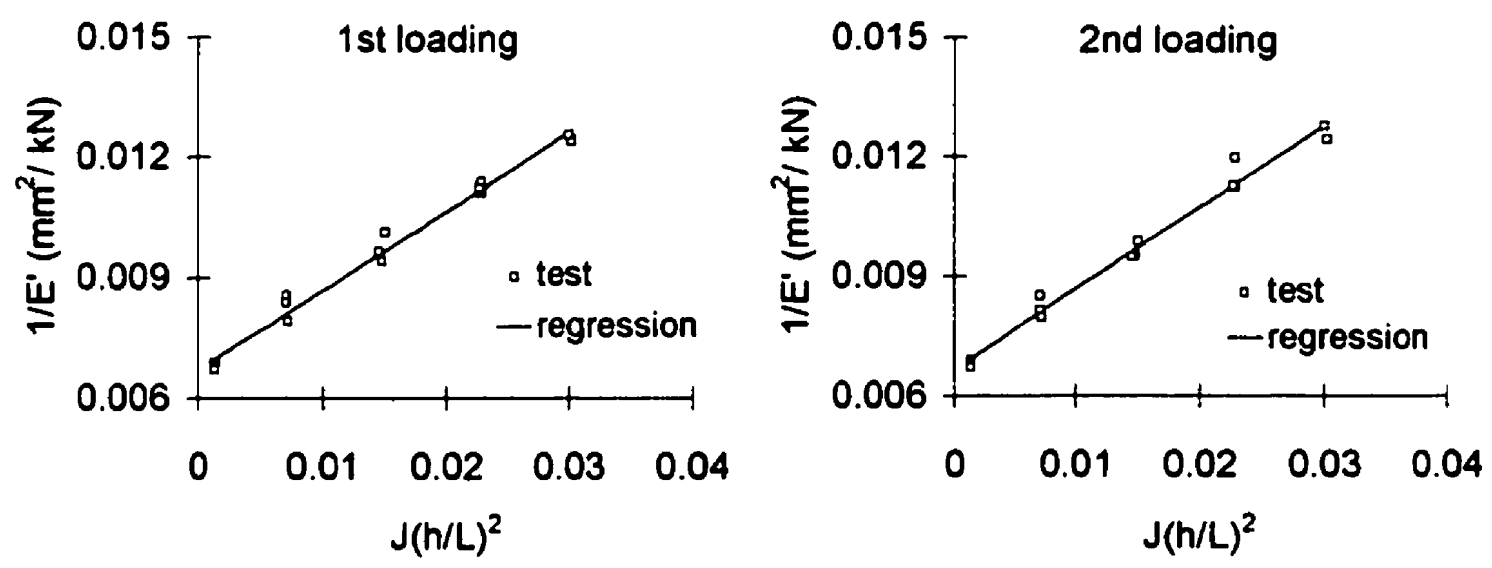

Figure 7.4 Plot of $1 / E^{\prime}$ versus $J(h / L)^{2}$ and the material characteristic line for GR-4 graphite/epoxy specimens. 
Table 7.2 Values of $G_{13}$ and $E_{11}$ for different sets of the graphite/epoxy specimens obtained by the VSM (GPa).

\begin{tabular}{|c|c|c|c|c|c|c|c|c|}
\hline \multirow[b]{2}{*}{ Set ID. } & \multicolumn{4}{|c|}{ First loading } & \multicolumn{4}{|c|}{ Second loading } \\
\hline & GR-1 & GR-2 & GR-3 & GR-4 & GR-1 & GR-2 & GR-3 & GR-4 \\
\hline$E_{11}$ & 151.9 & 150.8 & 155.8 & 149.4 & 151.0 & 153.1 & 156.4 & 151.0 \\
\hline$G_{13}$ & 4.55 & 4.59 & 4.32 & 5.07 & 4.87 & 4.7 & 4.44 & 4.88 \\
\hline
\end{tabular}
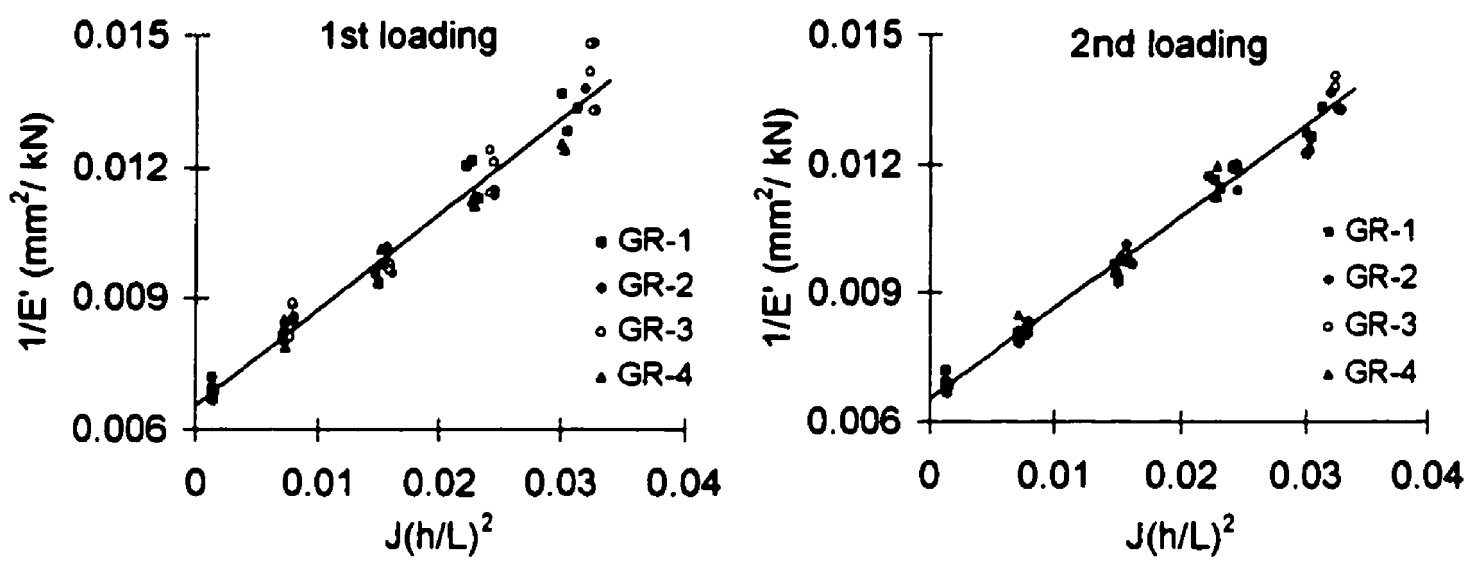

Figure 7.5 Plot of $1 / E^{\prime}$ versus $J(h / L)^{2}$ for all graphite/epoxy sets.

Four specimens of graphite/epoxy were used to evaluate the longitudinal modulus by the tensile test. Two extensometers, with $25.4 \mathrm{~mm}$ gage lengths, were attached back to back on each specimen to measure the elongation of the specimens during the test. The longitudinal moduli obtained from these tests were 149.8, 146.7, 153.3 and $150.9 \mathrm{GPa}$ giving an average value of $150.2 \mathrm{GPa}$.

To evaluate the shear modulus of the material by the $\pm 45^{\circ}$ and the Iosipescu shear tests, three specimens were used for each method. In the $\pm 45^{\circ}$ shear test the longitudinal strain was measured by attaching two $25.4 \mathrm{~mm}$ gage length extensometers to the two surfaces of the specimens while the transverse strain was measured by strain gages. The shear moduli obtained from these tests were $4.86,5.0$ and $4.76 \mathrm{GPa}$ for the $\pm 45^{\circ}$ shear test, and 5.83 , 6.11 and $6.51 \mathrm{GPa}$ for the Iosipescu shear test for average values of 4.87 and $6.15 \mathrm{GPa}$, 
respectively.

Table 7.3 Summary of the results obtained from different test methods and $95 \%$ confidence intervals for graphite/epoxy (GPa).

\begin{tabular}{cccccc}
\hline & $\begin{array}{c}\text { lst loading } \\
\text { VSM }\end{array}$ & $\begin{array}{c}\text { 2nd loading } \\
\text { VSM }\end{array}$ & Tensile test & $\begin{array}{c} \pm 45^{\circ} \text { shear } \\
\text { test }\end{array}$ & $\begin{array}{c}\text { Iosipescu } \\
\text { shear test }\end{array}$ \\
\hline$E_{11}$ & $152.4 \pm 4.4$ & $153.1 \pm 2.8$ & 150.8 & - & - \\
$G_{13}$ & $4.57 \pm 0.21$ & $4.69 \pm 0.15$ & - & - & - \\
$G_{12}$ & - & - & - & 4.87 & 6.15 \\
\hline
\end{tabular}

Table 7.3 summarizes the results obtained from the various test methods. The values of $E_{11}$ and $G_{13}$ of the VSM in this table were evaluated from the results of the tests on all the specimens (Fig. 7.5). The $95 \%$ confidence intervals are also reported for the results obtained by the VSM. The small margin of the confidence intervals confirm the reliability of the proposed method. The difference between the values of longitudinal moduli obtained by the tensile test and the VSM is less than $2 \%$, indicating a good agreement. The value of $G_{12}$ obtained by the $\pm 45^{\circ}$ shear test and the $G_{13}$ values obtained by the VSM are also in good agreement. Nevertheless, as was mentioned earlier, the $G_{13}$ and $G_{12}$ values of the materials may not be equal due to different distribution of the fibers through-thethickness and through-the-width of the specimens. Moreover, in hand lay-up materials, $G_{12}$ is expected to be larger than $G_{13}$. The results obtained from the losipescu shear test are considerably higher than the other values. This difference, as mentioned in the ASTM 5379-93, can be attributed to the nature of this method which usually produces higher value for shear modulus when highly anisotropic $0^{\circ}$ specimens are used. Finally, it should be emphasized that the fixture fabricated for the VSM is in its preliminary design stage. Refinement and enhancement of the fixture is expected to further improve the results.

\subsection{Investigation on Kevlar/epoxy}

The specimens of Kevlar/epoxy were cut from a pulltruded panel with average thickness of $1.9 \mathrm{~mm}$. The panel was provided by TW Pultrusion Ltd of Dartmouth, NS. The 
specimens were prepared for the VSM, $10^{\circ}$ off-axis and Iosipescu shear tests. The tensile properties of the material had been provided by the supplier of the material. The width of the specimens was about $12.5 \mathrm{~mm}$ for the VSM and $13 \mathrm{~mm}$ for the $10^{\circ}$ off axis. The dimensions of the specimens are presented in Appendix I. No sanding or polishing was applied to the surfaces of the specimens.

Six sets of specimens were subjected to the VSM, with the $L / h$ varing between 7.02 and 30.6. There were at least 15 specimens in each set for five different spans. The speed of the tests for each set was calculated based on the strain rate specifications of Table 7.4. Similar to the previous case, the specimens of Kevlar/epoxy were tested twice. The loaddeflection curves of the tests are presented in Appendix J. The initial slopes of the loaddeflection curves are also presented in Appendix I. These values were used to draw the plots of $1 / E^{\prime}$ versus $J(h / L)^{2}$ for each set of specimens in Figs 7.6 through 7.11. The value of $\alpha$ for all cases was taken to be 0.7 . Also shown in these figures are the characteristic lines of each sets which were determined by linear regression analysis. The $E$ and $G$ values of each set were determined from the reciprocal of the intercepts and slopes of the characteristic lines, respectively. The values are tabulated in Table 7.5. The plot of $1 / E^{\prime}$ versus $J(h / L)^{2}$ for all sets are shown in Fig. 7.12.

Table 7.4 Specifications of strain rate cases for different sets of Kevlar/epoxy specimens.

\begin{tabular}{ccccc}
\hline Designation & Equation & $E / G$ & $\dot{\varepsilon}_{\max }$ & $\dot{\gamma}_{\text {ar }}$ \\
\hline $\mathrm{K}-1$ & 6.32 & 30 & 0.01 & var. \\
$\mathrm{K}-2$ & 6.32 & 30 & 0.01 & var. \\
$\mathrm{K}-3$ & 6.32 & 10 & 0.01 & var. \\
$\mathrm{K}-4$ & 6.32 & 10 & 0.01 & var. \\
$\mathrm{K}-5$ & 6.33 & 30 & var. & 0.01 \\
$\mathrm{~K}-6$ & 6.33 & 30 & var. & 0.01 \\
\hline
\end{tabular}



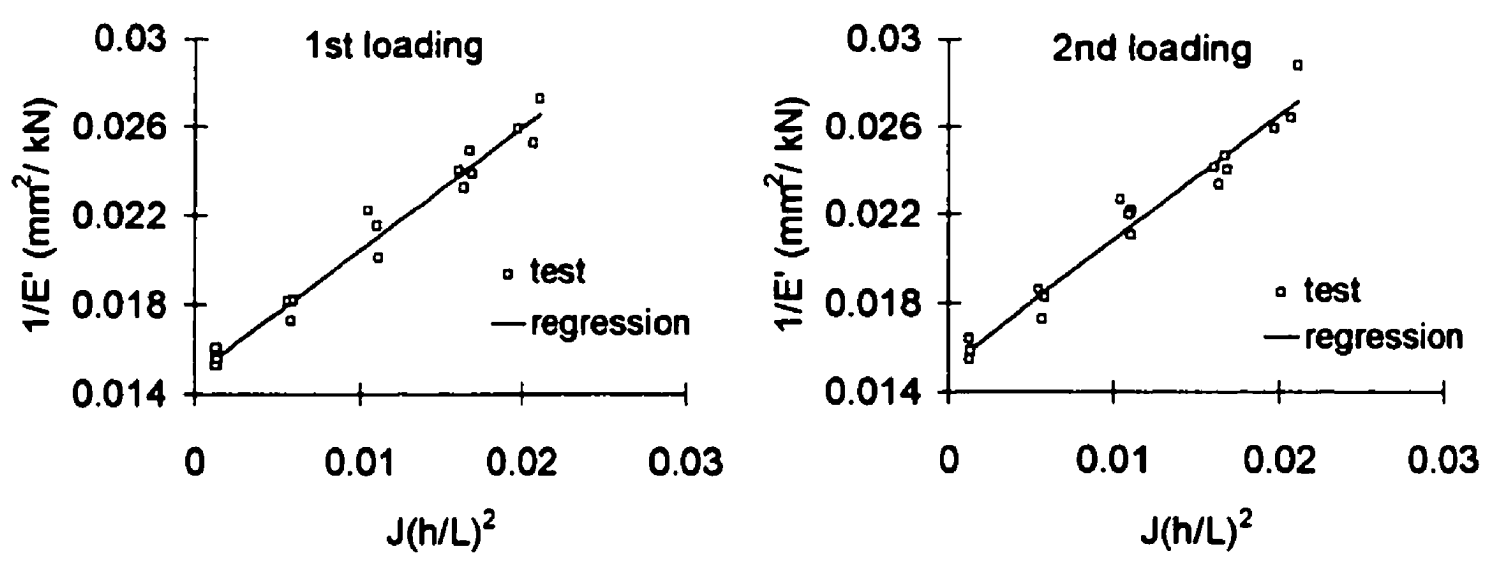

Figure 7.6 Plot of $1 / E^{\prime}$ versus $J(h / L)^{2}$ and the material characteristic line for K-1 Kevalr/epoxy specimens.
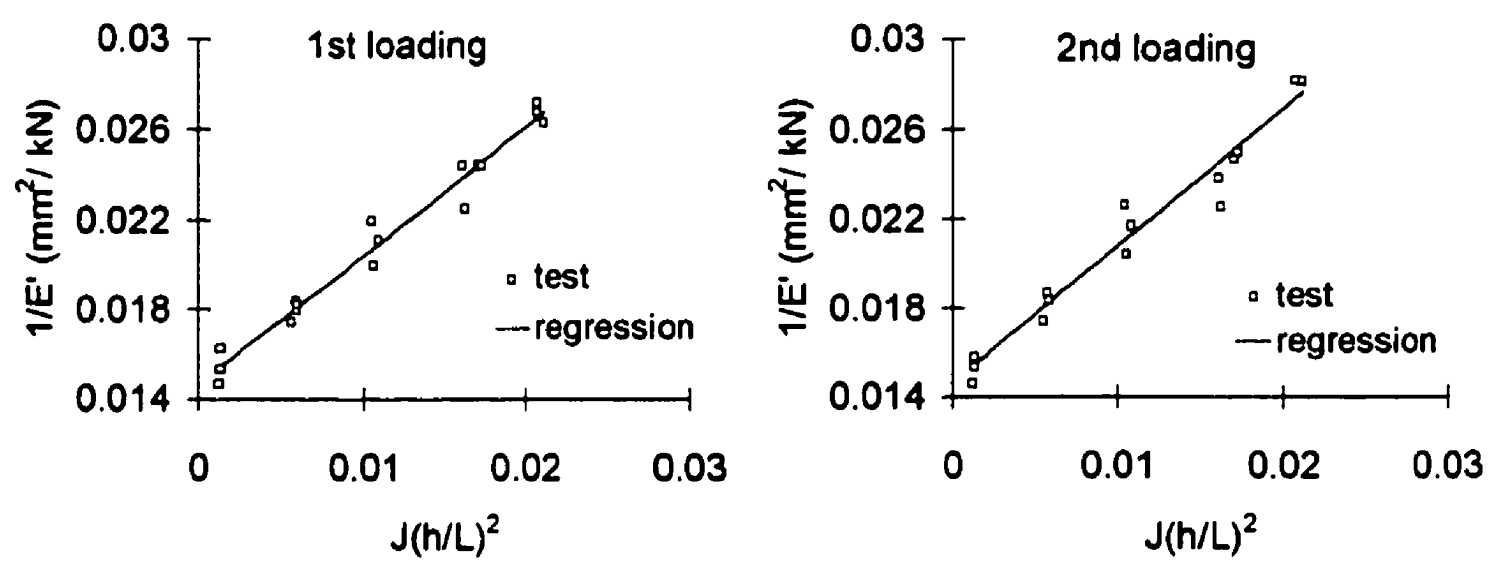

Figure 7.7 Plot of $1 / E^{\prime}$ versus $J(h / L)^{2}$ and the material characteristic line for K-2 Kevalr/epoxy specimens. 

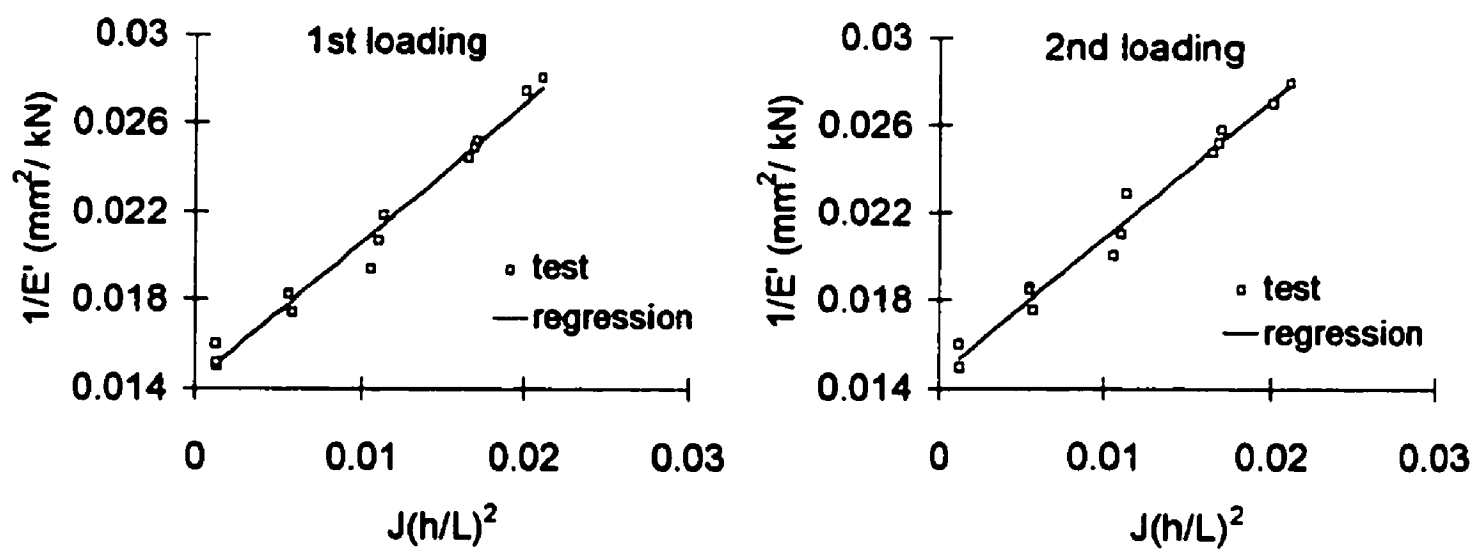

Figure 7.8 Plot of $1 / E^{\prime}$ versus $J(h / L)^{2}$ and the material characteristic line for K-3 Kevalr/epoxy specimens.
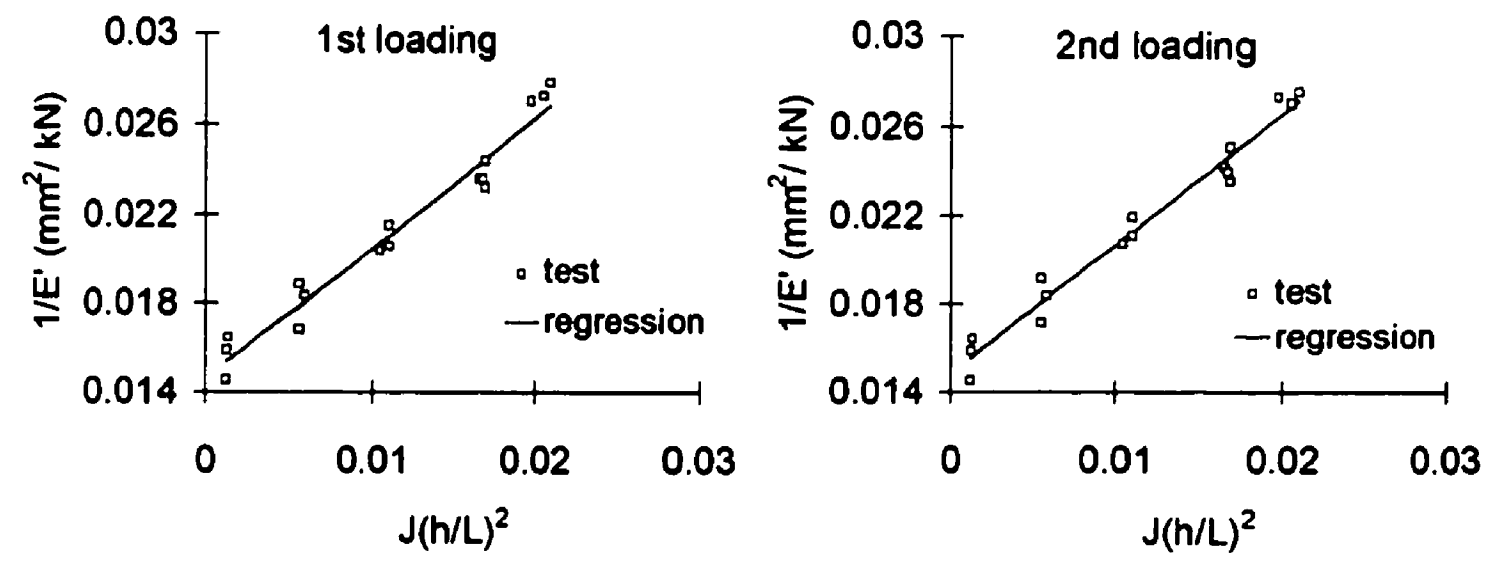

Figure 7.9 Plot of $1 / E^{\prime}$ versus $J(h / L)^{2}$ and the material characteristic line for K-4 Kevalr/epoxy specimens. 

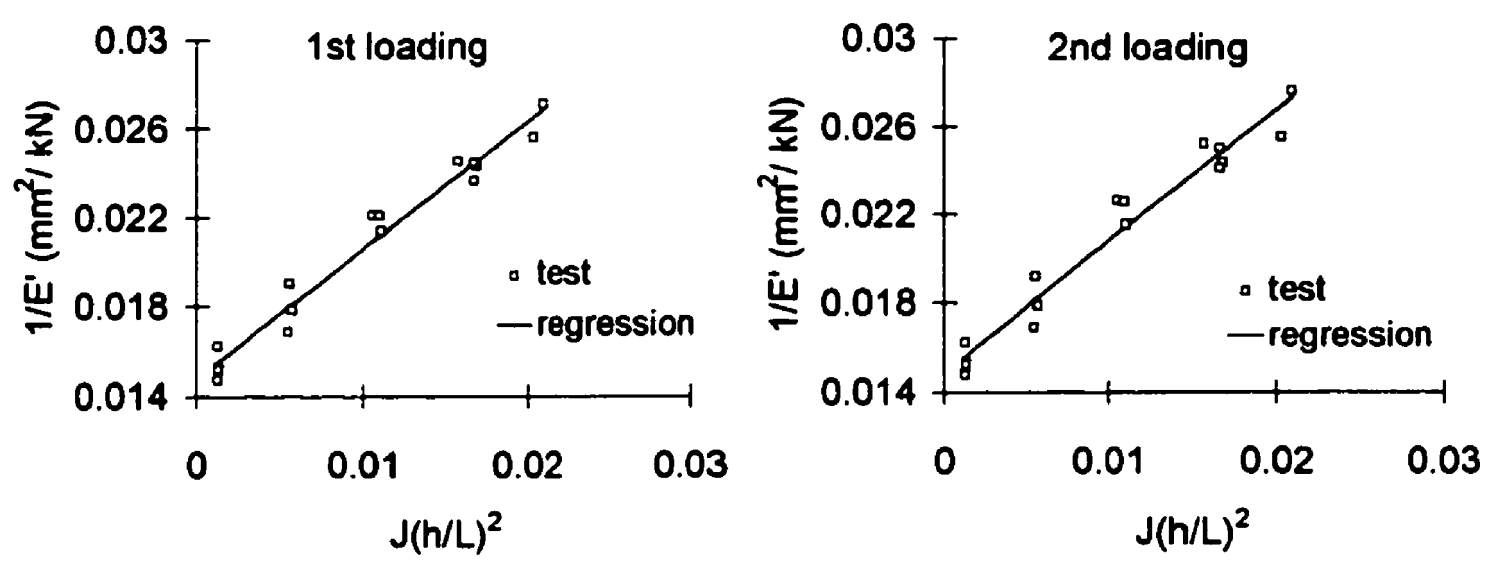

Figure 7.10 Plot of $1 / E^{\prime}$ versus $J(h / L)^{2}$ and the material characteristic line for K-5 Kevalr/epoxy specimens.
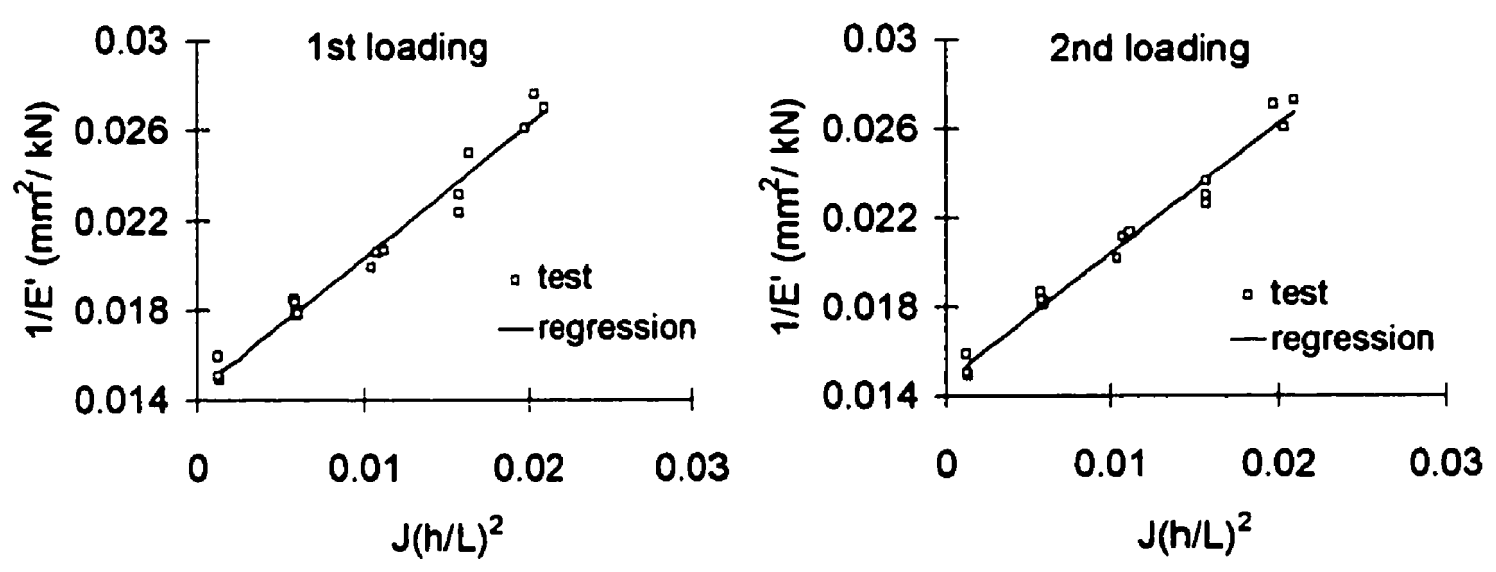

Figure 7.11 Plot of $1 / E^{\prime}$ versus $J(h / L)^{2}$ and the material characteristic line for K-6 Kevalr/epoxy specimens. 
Table 7.5 Values of $G_{13}$ and $E_{11}$ for different sets of the Kevlar/Epoxy specimens obtained by the $\operatorname{VSM}(\mathrm{GPa})$.

\begin{tabular}{|c|c|c|c|c|c|c|c|c|c|c|c|c|}
\hline \multirow[b]{2}{*}{ Set ID } & \multicolumn{6}{|c|}{ First loading } & \multicolumn{6}{|c|}{ Second loading } \\
\hline & $\mathrm{K}-1$ & $\mathrm{~K}-2$ & $\mathrm{~K}-3$ & $\mathrm{~K}-4$ & K-5 & K-6 & K-1 & K-2 & $\mathrm{K}-3$ & $\mathrm{~K}-4$ & $\mathrm{~K}-5$ & K-6 \\
\hline$E_{11}$ & 66.9 & 67.9 & 69.7 & 68.3 & 67.6 & 69.4 & 66.0 & 68.4 & 68.9 & 67.6 & 67.4 & 68.3 \\
\hline$G_{13}$ & 1.82 & 1.75 & 1.59 & 1.71 & 1.73 & 1.68 & 1.76 & 1.62 & 1.58 & 1.69 & 1.68 & 1.73 \\
\hline
\end{tabular}
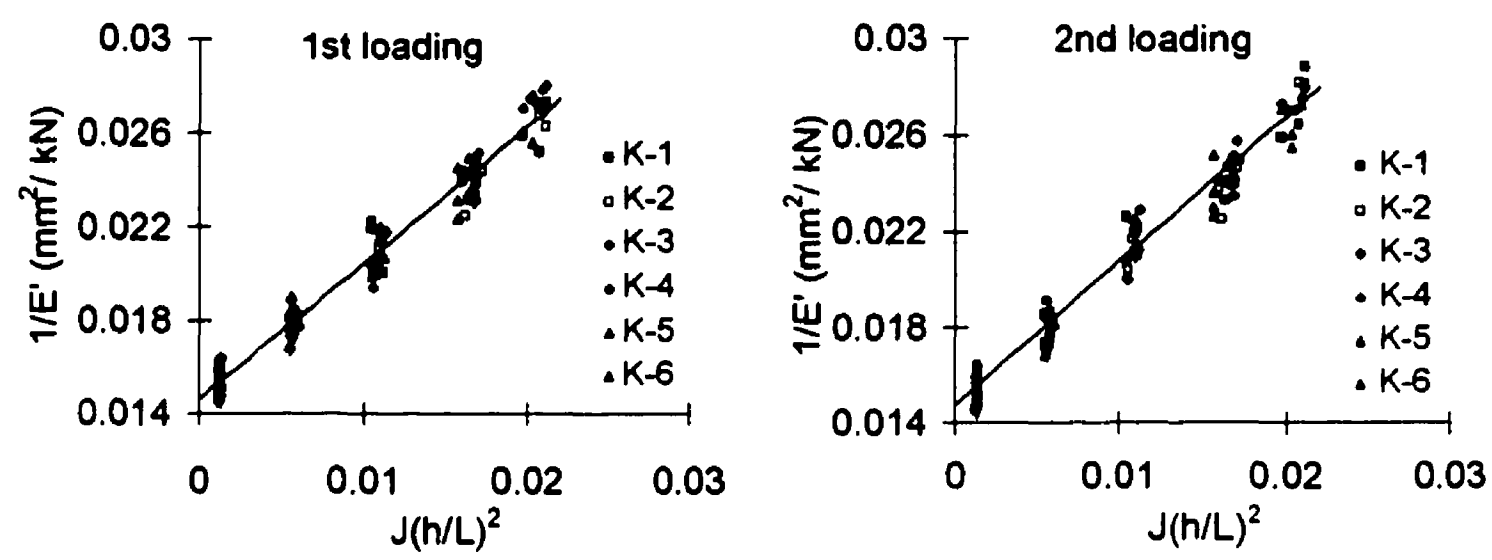

Figure 7.12 The plot of $1 / E^{\prime}$ versus $J(h / L)^{2}$ for all sets of Kevlar/epoxy specimens.

As can be seen in Figs 7.6 through 7.11 , the distribution of the data points in the $1 / E^{\prime}$ versus $J(h / L)^{2}$ coordinate system closely follows a linear pattern. As a result, one can conclude that the VSM is applicable to the Kevlar/epoxy composite. The $E$ and $G$ values obtained from different sets of specimens are also quite close to each other and do not show dependency on the strain rate cases of Table 7.4. Figure 7.12, which shows the scatter of the data points for all specimen sets, also does not indicate a dependency on the strain rate cases. As a result, the variation of strain rate for specimens with different $L h$ is not a problem for this material.

The values of the tensile modului, as provided by TW Pultrosion Ltd., were $67.8,66.6$ and $65.3 \mathrm{GPa}$ with an average of $66.6 \mathrm{GPa}$. The panel used to obtain the tensile test specimens was the same one that later was used for the VSM, the $10^{\circ}$ off-axis and the Iosipescu 
tests. To evaluate the $G_{12}$ value of the material, three specimens were used for the Iosipescu and two specimens were used for the $10^{\circ}$ off-axis shear tests. The $G_{12}$ values obtained from these tests were 2.09, 2.13 and $2.24 \mathrm{GPa}$ for the Iosipescu shear tests, and 1.86 and $1.72 \mathrm{GPa}$ for the $10^{\circ}$ off-axis shear tests giving average values of 2.15 and 1.79 $\mathrm{GPa}$, respectively.

Table 7.6 Summary of the results obtained from different test methods and $95 \%$ confidence intervals for Kevlar/epoxy (GPa).

\begin{tabular}{cccccc}
\hline & $\begin{array}{c}\text { l st loading } \\
\text { VSM }\end{array}$ & $\begin{array}{c}\text { 2nd loading } \\
\text { VSM }\end{array}$ & tensile test & $\begin{array}{c}10^{\circ} \text { off-axis } \\
\text { shear test }\end{array}$ & $\begin{array}{c}\text { Iosipescu } \\
\text { shear test }\end{array}$ \\
\hline $\mathrm{E}_{11}$ & $68.3 \pm 1.3$ & $67.8 \pm 1.3$ & 66.6 & - & - \\
$\mathrm{G}_{13}$ & $1.72 \pm 0.06$ & $1.68 \pm 0.07$ & - & - & - \\
$\mathrm{G}_{12}$ & - & - & - & 1.79 & 2.15 \\
\hline
\end{tabular}

The summary of the various tests results are tabulated in Table 7.6. The material characteristic line fitted to the results of all the VSM specimens (Fig. 7.12) was used for the determination of $E_{11}$ and $G_{13}$ values in this table. The $95 \%$ confidence intervals reported for the VSM results are very small. This confirms the integrity and reliability of the method for the evaluation of $E$ and $G$ values for the Kevlar/epoxy composite. The difference between the values of longitudinal moduli obtained by the tensile test and the VSM is quite small. Recalling the earlier discussion on the difference between $G_{12}$ and $G_{13}$, the values of $G_{13}$ obtained by the VSM are quite close to the $G_{12}$ value obtained by the $10^{\circ}$ off-axis shear test. Nevertheless, as it was discussed in Chapter 2 , one should expect an overestimated value for shear modulus from the $10^{\circ}$ off-axis method, unless relatively long specimens are used. The values from the losipescu shear test are considerably higher than the other values. This difference, as mentioned in the previous section, can be attributed to the nature of this method which usually overestimates the shear modulus when highly anisotropic $0^{\circ}$ specimens are used. The errors introduced by the VSM fixture due to possible fabrication imperfection can also be considered as another source for discrepancy. 


\subsection{Investigation on E-glass/epoxy}

The specimens of E-glass/epoxy were subjected to the VSM, tensile and Iosipescu shear tests. The specimens were cut from pulttruded bars provided by Glasforms Inc., San Jose, California, with an average thickness of $6.3 \mathrm{~mm}$. The average width of the specimens was about $13.4 \mathrm{~mm}$ for the VSM and $14 \mathrm{~mm}$ for the tensile test. The exact dimensions of the specimens are presented in Appendix I. Similar to the Kevlar/epoxy specimens, no sanding or polishing was applied to the surfaces of the specimens.

For the VSM tests, four sets of specimens were prepared. There were 12 specimens in each set which were tested at 4 different spans. The $L / h$ of the specimens varied between $4.93 \mathrm{~mm}$ and $17.82 \mathrm{~mm}$. The speed of the tests for each set was calculated based on the strain rate specifications of Table 7.7. Similar to the previous cases, the specimens of Eglass/epoxy were tested twice. The load-deflection curves of the tests are presented in Appendix J with the initial slopes reported in Appendix I. The values of the initial slopes were used to draw the plots of $1 / E^{\prime}$ versus $J(h / L)^{2}$ for each sets of specimens. These plots are presented in Figs 7.13 through 7.16. Similar to the previous cases, $\alpha=0.7$ was used for all sets of the specimens. The characteristic lines of each set, determined by linear regression analysis, are also drawn in the figures. The $E$ and $G$ values of each set, determined respectively from the reciprocal of the intercepts and slopes of the characteristic lines, are tabulated in Table 7.8. The plot of $1 / E^{\prime}$ versus $J(h / L)^{2}$ containing the results of tests for all sets of specimens are also shown in Fig. 7.17.

Table 7.7 Specifications of strain rate cases for different sets of E-glass/epoxy specimens.

\begin{tabular}{ccccc}
\hline Designation & Equation & $E / G$ & $\dot{\varepsilon}_{\max }$ & $\dot{\gamma}_{\mathrm{ar}}$ \\
\hline GL-1 & 6.32 & 30 & 0.01 & var. \\
GL-2 & 6.32 & 10 & 0.01 & var. \\
GL-3 & 6.32 & 10 & 0.01 & var. \\
GL-4 & 6.33 & 10 & var. & 0.01 \\
\hline
\end{tabular}



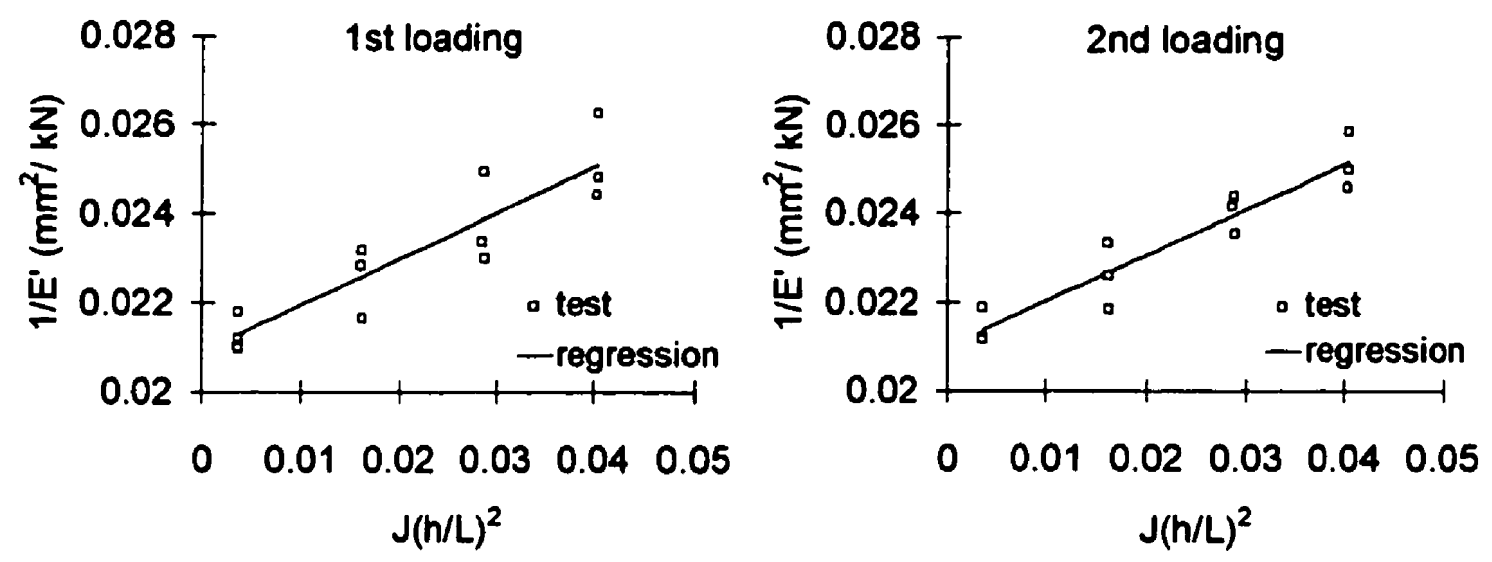

Figure 7.13 Plot of $1 / E^{\prime}$ versus $J(h / L)^{2}$ and the material characteristic line for GL-l E-glass/epoxy specimens.
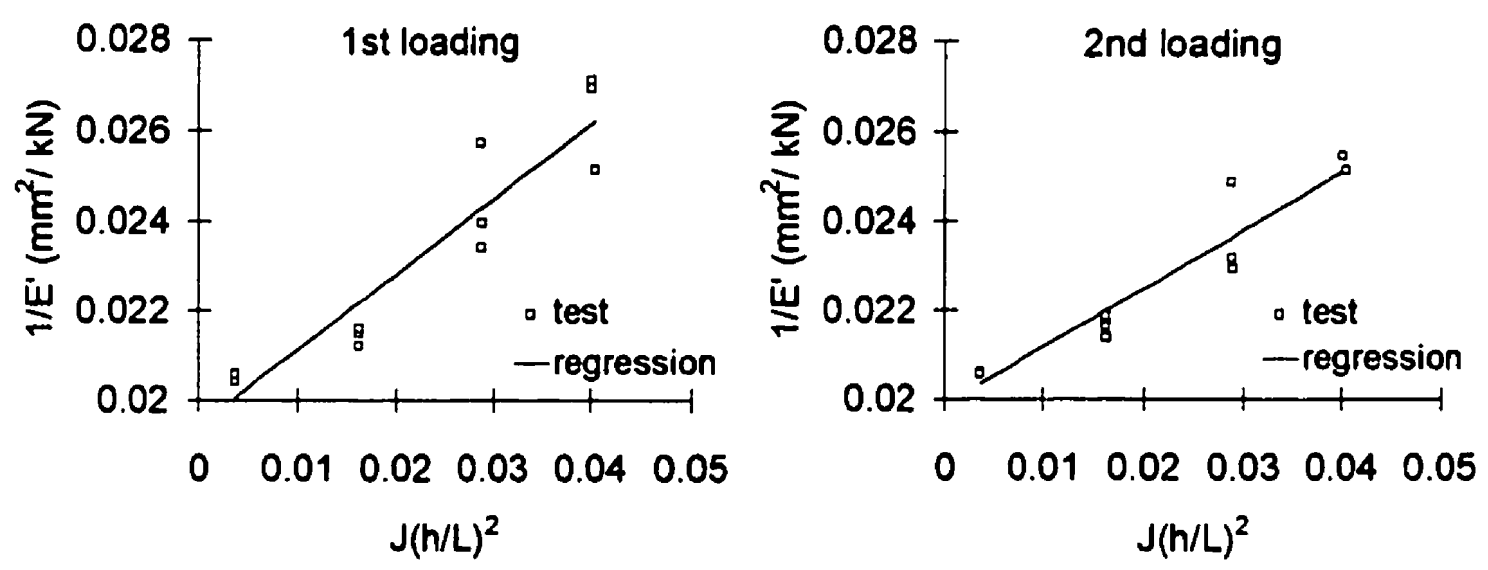

Figure 7.14 Plot of $1 / E^{\prime}$ versus $J(h / L)^{2}$ and the material characteristic line for GL-2 E-glass/epoxy specimens. 

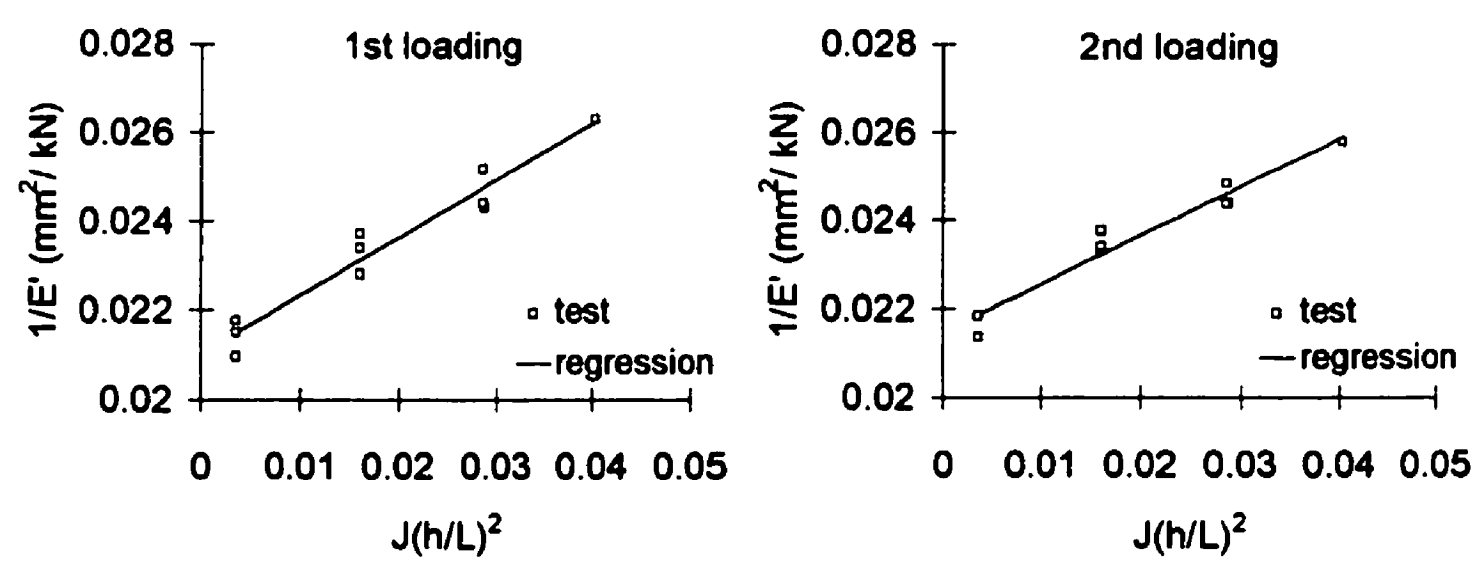

Figure 7.15 Plot of $1 / E^{\prime}$ versus $J(h / L)^{2}$ and the material characteristic line for GL-3 E-glass/epoxy specimens.
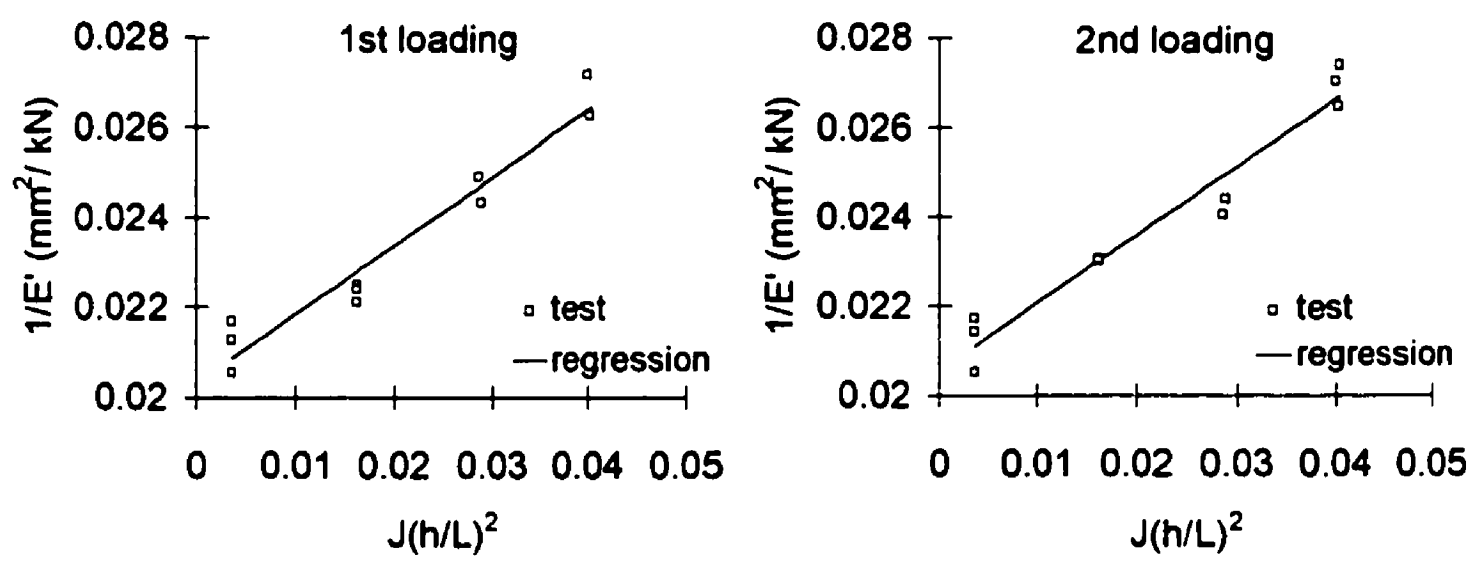

Figure 7.16 Plot of $1 / E^{\prime}$ versus $J(h / L)^{2}$ and the material characteristic line for GL-4 E-glass/epoxy specimens. 
Table 7.8 Values of $G_{13}$ and $E_{11}$ for different sets of the E-glass/epoxy specimens obtained by the VSM (GPa).

\begin{tabular}{|c|c|c|c|c|c|c|c|c|}
\hline \multirow[b]{2}{*}{ Set ID } & \multicolumn{4}{|c|}{ First loading } & \multicolumn{4}{|c|}{ Second loading } \\
\hline & GL-1 & GL-2 & GL-3 & GL-4 & GL-1 & GL-2 & GL-3 & GL-4 \\
\hline$E_{11}$ & 47.8 & 51.4 & 47.5 & 49.2 & 47.6 & 50.3 & 46.6 & 48.7 \\
\hline $\mathrm{G}_{13}$ & 9.72 & 5.98 & 7.75 & 6.56 & 9.78 & 7.66 & 9.09 & 6.57 \\
\hline
\end{tabular}

A linear trend between $1 / E^{\prime}$ and $J(h / L)^{2}$ is evident from Figs 7.13 through 7.17. For this material, the deviation of the data points from the regression lines appears to be significant. A closer examination of the figures reveals that the relative deviation (ratio of deviation to the measured value) is in the same range of those of Kevlar/epoxy and graphite/epoxy. However, the range of $1 / E^{\prime}$ in this case is considerably smaller than for the other two materials. That is, the ratio of the maximum $1 / E^{\prime}$ to the minimum $1 / E^{\prime}$ in the E-glass/epoxy tests is less than 1.3, while the same ratio for the Kevlar/epoxy and the graphite/epoxy tests results is lager than 2. As a result, a usual variation of 5 to 10 percent in the material properties appears as a significant discrepancy in Figs 7.13 through 7.17 for the present case. This problem, as was discussed in Chapter 6 , is due to the small $E / G$ value of E-glass/epoxy which makes the VSM less efficient. Consequently, the values of $G$ obtained from different sets of specimens do not agree with each other very well. A wider range in $1 / E^{\prime}$ could be obtained by testing specimens with wider range of $L h$.

The $G$ values of Table 7.8 for second loading and also the plots of the second loading test results in Fig. 7.17 indicate that the results of the GL-4 set of specimens are considerably different from those of other sets. This difference may be interpreted as the influence of the strain rate sensitivity of E-glass/epoxy in the VSM. However, a more detailed investigation, as follows, would imply that the reason for the difference must be attributed to the variation of the material properties and the other variables involved with testing and estimating the initial slopes of the load-deflection curves. 
Referring to Fig. 7.17, the $E^{\prime}$ values obtained for the second loading of GL-4 specimens are considerably smaller than those of other sets for tests with the smallest span (31.19 $\mathrm{mm}$ ). Obviously, this difference is the reason for the small value of $G$ obtained from this set of specimens. By checking Appendix J, one will realize that the speed of tests for GL-4 specimens was between those of GL-1, GL-2 and GL-3 specimens. As a result, in case of sensitivity to strain rate, the $E^{\prime}$ values of GL-4 specimens should have fallen between the $E^{\prime}$ values obtained from the other sets of the specimens. Since this was not the case, the existing difference cannot be related to the strain rate sensitivity of the material.

The $G$ value of the GL-1 set of specimens in Table 7.8 for first loading is also considerably higher than the $G$ values of the other sets. Since the plot in Fig. 7.17 of the first loading does not show an obvious dependence of the GL-1 specimens to strain rate, one can again attribute the higher value of $G$ to the variation of the material properties and the other variables involved with testing and estimating the initial slopes of the load-deflection curves.
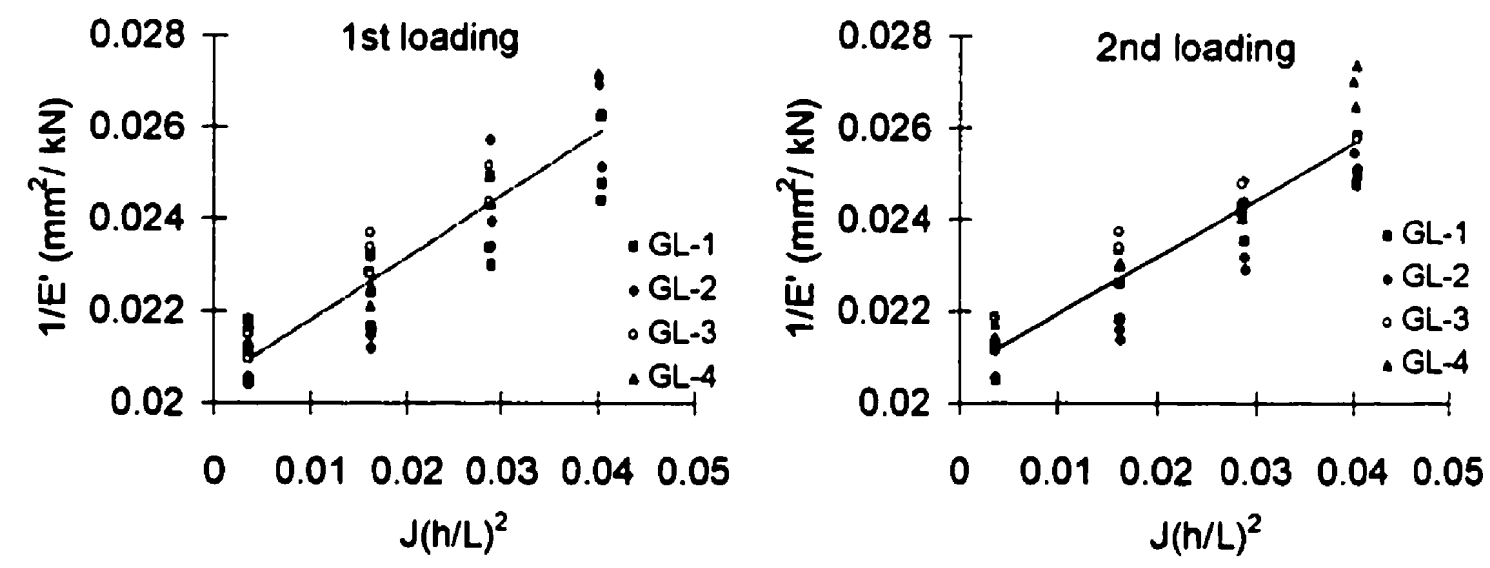

Figure 7.17 Plot of $1 / E^{\prime}$ versus $J(h / L)^{2}$ for all sets of E-glass/epoxy specimens.

The tensile test was conducted on three specimens. The $E$ values obtained from these specimens were $47.2,50.61$ and $47.7 \mathrm{GPa}$ with an average value of $48.5 \mathrm{GPa}$. The result 
from the Iosipescu shear tests were $7.07,7.39$ and 8.27 with an average value of 7.57 GPa. These average values, along with the $E$ and $G_{13}$ obtained from the characteristic line of all the VSM specimens (Fig. 7.17), are tabulated in Table 7.9. The $95 \%$ confidence intervals for the results of the VSM are also determined by statistical means and reported in the same table. The small confidence interval for the $E$ value confirms the reliability of the VSM method for evaluation of the E-glass/epoxy's longitudinal modulus. The $95 \%$ interval for the shear modulus is more than $10 \%$ of the modulus itself which is considerably higher than the values obtained for the Kevlar/epoxy and the graphite/epoxy investigated earlier. The cause of this problem, as was discussed earlier, is due to the small range in $1 / E^{\prime}$ for the tests. The consistency of the results could be improved by increasing the range of $L / h$ of the specimens. Nevertheless, both values of $E$ and $G$ calculated from the VSM test results on all sets of specimens, agree very well with the values obtained by the tensile and the Iosipescu shear tests.

Table 7.9 Summary of the results obtained from different test methods and $95 \%$ confidence intervals for E-glass/epoxy (GPa).

\begin{tabular}{ccccc}
\hline & $\begin{array}{c}\text { 1st loading } \\
\text { VSM }\end{array}$ & $\begin{array}{c}\text { 2nd loading } \\
\text { VSM }\end{array}$ & tensile test & $\begin{array}{c}\text { Iosipescu } \\
\text { shear test }\end{array}$ \\
\hline$E_{11}$ & $48.9 \pm 1.1$ & $48.3 \pm 1.0$ & 48.5 & - \\
$G_{13}$ & $7.41 \pm 1.03$ & $8.06 \pm 1.09$ & - & - \\
$G_{12}$ & - & - & - & 7.57 \\
\hline
\end{tabular}

\subsection{Summary and conclusions}

In this chapter, the integrity of the VSM was experimentally investigated. For this, three $0^{\circ}$ unidirectional FRPC, including graphite/epoxy, Kevlar/epoxy and E-glass/epoxy, were considered. The longitudinal and the through-the-thickness shear moduli of the materials were evaluated by subjecting them to the VSM test. The longitudinal moduli obtained by the VSM were verified by conducting the ASTM D3039-93 tensile test on the materials. Since there was not a simple method to evaluate the through-the-thickness shear moduli of 
the materials, the in-plane shear moduli were evaluated by common available shear test methods to assess the values obtained by the VSM. For this, the Iosipescu shear test was used on all materials while the $\pm 45^{\circ}$ shear test was applied only to the specimens of graphite/epoxy and the $10^{\circ}$ off-axis test was applied to the Kevlar/epoxy specimens. With the exception of the Iosipescu test results for graphite/epoxy and Kevlar/epoxy, which were about $30 \%$ higher than the shear moduli obtained by the VSM, the values obtained by the different test methods were in good agreement. The higher values obtained from the Iosipescu test method for graphite/epoxy and Kevlar/epoxy were attributed to the nature of the test method which is known to generally overestimate the shear modulus of highly anisotropic $0^{\circ}$ specimens. The small discrepancies for other cases is quite acceptable for practical purposes. Nevertheless the discrepancy can be attributed to the following parameters.

a) The results obtained by the tensile test can be influenced by possible bending due to specimen and/or system misalignment.

b) The shear modulus obtained by the VSM method is $G_{13}$, however the results obtained from the other methods are $G_{12}$. As explained, these values are not necessarily equal.

c) None of the shear test methods used here are considered to be exact.

d) The $10^{\circ}$ off-axis shear test generally gives higher values, unless when applied to specimens with very large aspect ratios.

e) The quality of the material may not be uniform for all specimens.

f) None of the test methods has an exact procedure for the determination of the modulus value from the test data.

g) The fabricated fixture for the VSM is in its preliminary stage; further modification and enhancements of the fixture may improve the results. 
The effect of variable strain rate for specimens with different $L / h$, which is inherent in this method, was also investigated by performing tests under different strain rate cases. No apparent dependency was observed between the results obtained for different cases. Consequently, one can conclude that the non-constant strain rate in the VSM is not an issue for the materials tested. Based on the results of the VSM on all the specimens of each material, the $95 \%$ confidence intervals for the $E$ and $G$ values were statistically determined. With the exception for the $G$ value of the E-glass/epoxy, the $95 \%$ confidence intervals were quite small, confirming the reliability of the VSM. The larger confidence interval obtained for the shear modulus of the E-glass/epoxy was due to the small range in $1 / E^{\prime}$ over the VSM test results, which in turn was due to the low $E / G$ value of this material. Selecting a wider range of $L / h$ for the specimens is believed to improve the results. 


\section{Chapter 8}

\section{SUMMARY AND RECOMMENDATIONS}

A novel test method called the "Varying-Span Method" (VSM) was introduced and was extensively discussed in the previous chapters. Every effort was made to study and investigate the VSM from various angles so that one could recognise its advantages and its limitations. A summary of the investigation is presented below. Recommendations for future work and further development of the method are also provided in this chapter. Despite the extensive effort carried out, the author believes that the subject is still in its preliminary stage and requires further research and development.

\subsection{Summary}

A new test method capable of simultaneous evaluation of the longitudinal and throughthe-thickness shear moduli of FRPC was introduced in this thesis. With the new test method specimens with different spans were subjected to three-point bending. Therefore, the method was called the "Varying-Span Method" (VSM). The importance of evaluating the elastic properties of FRPC as an orthotropic material in different directions and the corresponding nomenclatures were presented in Chapter 1. Chapter 2 reviewed the common test methods available for the determination of the longitudinal and the shear moduli of FRPC. As a result of the review, it became clear that the VSM was a unique test method due to its capability of simultaneously evaluating two elastic moduli of a given FRPC while being a simple and efficient test method.

Chapter 3 provided the exact elasticity solution for three-point bending of orthotropic beams. This solution was used later to assess two approximate, yet simple solutions developed for treating the same problem. The approximate solutions sought for this problem were based on the through-the-thickness inextensibility and the Timoshenko 
beam theories. The first theory was discussed in Chapter 4 and references were given for the second.

The fundamentals of the VSM were presented in Chapter 5. It was shown that by determining the flexural stiffness of specimens with different length-to-depth ratio $(L / h)$, one could theoretically evaluate the longitudinal and through-the-thickness shear moduli of elastic materials, simultaneously. Chapter 6 showed that the efficiency of the method depended on the ratio of the longitudinal to shear moduli $(E / G)$ of the material, providing higher efficiency for materials with larger $E / G$. As a result, it was concluded that the method would be particularly applicable to FRPC. The theoretical and the experimental investigations in Chapters 6 and 7 confirmed that the VSM would be an efficient and a practical test method for this type of material.

The VSM's development, outlined in Chapter 5, was built on the two approximate theories introduced earlier, that is, the through-the-thickness inextensibility and the Timoshenko beam theories. Because of the simplicity of the second theory, the theoretical and the experimental investigations were limited to the use of this theory. In the solution developed based on the Timoshenko beam theory, a new quantity called the "apparent modulus of elasticity" was introduced. This quantity, as defined by Eqn (5.18), is determined from the result of three-point bending test. It was shown that when the apparent moduli of elasticity of specimens with different $L / h$ are plotted in the coordinates system of Fig. 5.3, a straight line called the "material characteristic line" is obtained. One could then evaluate the longitudinal and the shear moduli of the material from the reciprocal of the intercept and the slope of this line, respectively.

The distribution width of the applied load onto the specimen, and the supports reaction forces were defined by a parameter denoted by " $\alpha$ ". It was shown in Chapter 6 that $\alpha$ was a function of specimen geometry and material properties. The exact values of $\alpha$ were 
provided in Figs 6.8 through 6.18. It was also shown that for most practical cases, determination of the exact value of $\alpha$ was not necessary and the use of an approximate value of $\alpha=0.7$ would provide sufficient accuracy. This approximate value was later used in Chapter 7 for the experimental investigations.

The materials considered in the experimental investigations of Chapter 7 were $0^{\circ}$ unidirectional graphite/epoxy, Kevlar/epoxy and E-glass/epoxy. The longitudinal and shear moduli evaluated by the VSM were compared with the values obtained by the tensile test (ASTM D3039-93) and the $\pm 45^{\circ}$ (ASTM D3518-94), Iosipescu (ASTM D5379-93) and $10^{\circ}$ off-axis shear test methods. The results obtained by the VSM were in close agreement with those obtained from the other test methods. The small differences were attributed to the following factors:

a) The effect of the possible bending due to fiber, specimen and/or system misalignment in the tensile test.

b) The inherent difference between the quantity of the through-the-thickness shear modulus obtained by the VSM method and the in-plane shear modulus obtained by other shear test methods. These two shear moduli are not necessarily equal.

c) None of the shear test methods used is considered to be an exact method.

d) The overestimation of shear modulus by the $10^{\circ}$ off-axis shear test when specimens with very large aspect ratios are not used.

e) The variation of material properties from one specimen to another.

f) The fact that the procedure for the determination of $E$ and $G$ values from the tests results is not an exact procedure.

g) The imperfections in the VSM fixture due to its preliminary design and fabrication.

In Chapter 6, it was shown that maintaining a constant strain rate for both the longitudinal and shear strain when the specimens with different $L / h$ are used is not possible. This 
phenomenon prompted the question of "how accurate would the results of the VSM be if the materials were strain rate sensitive?". The influence of the strain rate was therefore evaluated by numerical and experimental investigations outlined in Chapters 6 and 7, respectively. The numerical investigation showed that the variation of the strain rate from one specimen to another would not impact the results of the VSM for common FRPC materials. The same conclusion was reached from the experimental observations in Chapter 7. Nevertheless, the necessary guidelines to reduce the possible effect of variable strain rates were provided in Chapter 6 .

The reliability of the VSM was assessed by determining the $95 \%$ confidence intervals for the $E$ and $G$ values evaluated by this method in Chapter 7. Except for the shear modulus of E-glass epoxy, the $95 \%$ confidence intervals were quite narrow, indicating the reliability of the results. The exception was the shear modulus $(G)$ of the E-glass epoxy whose $95 \%$ confidence interval was larger than $30 \%$ of the $G$ value. This was attributed to the low $E / G$ ratio of the material which did not produce sufficient change in the value of $1 / E^{\prime}$ obtained from the tests. A wider range of $L / h$ is believed to resolve the problem.

In conclusion, the VSM was shown to be a promising test method for evaluating the longitudinal and shear moduli of FRPC. The efficiency of the method increases for materials with large ratios of $E / G$. For a material with a small ratio of $E / G$, the predicted value of $G$ by the VSM becomes less reliable. However, from practical point of view, this is not of significance, since in a material with low $E / G$ ratio, the through-the-thickness shear modulus has insignificant role in effecting the behavior of the structure. Consequently, the importance of having an accurate value for the shear modulus fades.

In comparison to other available test methods, the VSM has significant advantages. In summary, these advantages are: 
a) The test specimens are subjected to a combined state of stresses which is representative of the in-service behavior of the material and is therefore more realistic.

b) The specimens can be extracted directly from the as-received structural components.

c) The specimens have simple rectangular geometry.

d) The specimens do not require special alteration (i.e. notches or holes).

e) Mounting of the specimens in the fixture is easy.

f) No strain gages and/or other expensive instruments are required.

g) The longitudinal and the through-the-thickness shear moduli are obtained simultaneously.

h) The properties obtained by the other methods are representative of the material behavior in a small gage length. In contrast, the properties obtained by the VSM reflects the response of the material in its entirety.

\subsection{Recommendations for future works and further development}

The limitations of time and resources usually do not allow one to reach to one's desired destination in research and science. However, while these are available, the spread of unknowns to be answered is so vast that they overwhelm one's capability. As a result, there must be others to continue and improve earlier works and accomplishments. The work presented in this thesis obviously is not an exception, and it follows the same rule. To keep the work started here going, a few recommendations for future work are provided below. In these recommendations different aspects of the VSM which require work and improvement are discussed.

i) Experimental investigations on other types of $0^{\circ}$ unidirectional FRPC: The experimental investigations in this thesis were limited to graphite/epoxy, Kevlar/epoxy and E-glass/epoxy having certain percentages of fibers. To validate the applicability of the VSM to other types of FRPC, experimentation on other composites, similar to the ones carried out in this thesis are recommended. 
ii) Application of the VSM to more general types of FRPC: The VSM can also be used for FRPC such as randomly oriented chopped-fiber, mat-fiber and woven-fiber composites. Additional experimental investigation, therefore, is recommended.

iii) Development of a fixture for the alignment shown in Fig 6.4a: All the theoretical and the experimental investigations in this thesis were based on the alignment of Fig. 6.4b. Moreover, the apparatus developed for the experiment was also designed for this purpose. As a result, the development of a fixture capable of holding a specimen based on the alignment of Fig. $6.4 \mathrm{a}$ and the corresponding investigations remain for future. This alignment will enable one to evaluate either $E_{11}$ and $G_{12}$ or $E_{22}$ and $G_{12}$ of the material, simultaneously. A state of plane stress always exists and specimens with very small $L / h$ can be tested. As a result, the shear modulus of materials with small $E / G$ values can be determined with sufficient accuracy.

iv) Extension of the VSM to dynamic testing: The same fundamentals used for simultaneous evaluation of the longitudinal and shear moduli of materials can be used to evaluate these properties by dynamic method. In the same vein, the method can be based on the effect of shear modulus on the flexural natural frequency of a composite beam. The effect of shear modulus increases for beams with small $L / h$ and for higher natural frequencies. Consequently, determining the first natural frequency of a specimen for at least two different $L / h$, or determining at least two different natural frequencies of the same span enables one to evaluate both the longitudinal and shear moduli of the material. For the evaluation of the two moduli from the test results, however, one needs to apply a beam theory which accounts for the shear effect. For this, use of the Timoshenko beam theory, despite its simplicity, is not recommended. This is due to the fact that the theory cannot provide the needed accuracy. This subject was discussed by Jalali and Taheri (1998b) and several others. Jalali and Taheri (1998b) showed that the through-the- 
thickness inextensibility theory provided very accurate values for the natural frequency of laminated beams. Therefore, this theory can efficiently be used for the suggested purpose. 


\section{REFERENCES}

Abdallah, M. G. and Gascoigne, H. E., (1989) The Influence of Test Fixture Design on the Iosipescu Shear Test for Fiber Composite Materials, Test Methods for Design Allowables for Fibrous Composites: 2nd Volume, ASTM STP 1003, American Society for Testing and Materials, pp. 231-260.

Adams, D. F. and Adams, L. G., (1990) Tensile Impact Tests of AS4/3501-6 and S2/3501-6 Unidirectional Composites and the 3501-6 Epoxy Matrix, J. Composite Materials, Vol. 24, pp. 256-269.

Adams, D. F. and Walrath, D. E., (1987) Current Status of the Iosipescu Shear Test Method, J. Composite Materials, Vol. 21, pp. 494-507.

Adams, D. F. and Lewis, E. Q., (1991) Influence of Specimen Gage Length and Loading Method on the Axial Compressive Strength of a Unidirectional Composite Materials, Experimental Mechanics, Vol. 31, pp. 14-20.

Adams, D. F. and Odom, E. M., (1991) Influence of Specimen Tabs on the Compressive Strength of a Unidirectional Composite Material, J. Composite Materials, Vol. 25, pp. 774-785.

Adist, N. R., (1983) Compression Testing of Graphite/Epoxy, Compression Testing of Homogeneous Materials and Composites, ASTM STP 808, American Society for Testing and Materials, pp. 175-186.

Arai, T. and Oku, T., (1979) Journal of Nuclear Materials, Vol. 79, pp. 227-234. 
Arcan, M., Hashin, Z. and Voloshin, A., (1978) A Method to produce Uniform PlaneStress States with Applications to Fiber-Reinforced Materials, Experimental Mechanics, Vol. 18, pp. 141-146.

Bansal, A. and Kumosa, M. (1995) Experimental and Analytical Studies of Failure Modes in Iosipescu Specimens Under Biaxial Loadings, J. Composite Materials, Vol. 29, pp. 334358.

Basar, Y., Ding, Y., and Schultz, R., (1993) Refined Shear-Deformation Models for Composite Laminated With Finite Rotations, Int. J. Solids and Structures, Vol. 30, pp. 2611-2638.

Berg, C. A., Tirosh, J. and Israeli, M. (1972) Analysis of Short Beam Bending of Fiber Reinforced Composites, Composite Material: Testing and Design, ASTM STP 497, American Society for Testing and Materials, pp. 206-218.

Bogetti, T. A., Gillespie, J. W., Jr. and Pipes, R. B., (1988) Evaluation of the IITRI Compression Test Method for Stiffness and Strength Determination, Composites Science and Technology, Vol. 32, pp. 57-76.

Broughton, W. R., Kumosa, M. and Hull, D., (1990) Analysis of the Iosipescu Shear Test as Applied to Unidirectional Carbon-Fiber Reinforced Composites, Composites Science and Technology, Vol. 38, pp. 299-325.

Bullock, R.E., (1974) Strength Ratio of Composite Materials in Flexure and in Tension, J. Composite Materials, Vol. 8, pp. 200-207. 
Chamis, C. C. and Sinclair, J. H., (1977) Ten-deg Off-Axis Test for Shear Properties in Fiber Composites, Experimental Mechanics, Vol. 17, pp. 339-346.

Chatterjee, S. N., (1996) Analysis of the Short-Beam Shear Test for Unidirectional Composites, Composite Materials: Testing and Design, ASTM STP 1274, American Society for Testing and Materials, pp. 320-339.

Chiao, T. T. and Hamstad, M. A., (1975) Testing of Fiber Composite Materials, Proceeding of the 1975 International Conference On Composite Materials, Vol. 2, pp. 884-915.

Chiao, C. C., Moore, R. L. and Chiao, T. T., (1977) Measurement of Shear Properties of Fiber Composites, Composites, Vol. 8, pp. 161-169.

Chow, T. S., (1971) On the Propagation of Flexural Waves in an Orthotropic Laminated Plate and Its Response to an Impulsive Load, J. Composite Materials, Vol. 5, pp. 306319.

Clements, L. L. and Chiao, T. T., (1977) Engineering Design Data for an Organic Fiber/epoxy Composite, Composites, Vol. 8, pp. 87-92.

Conti, P. and De Paulis, A., (1985) A Simple Model to Simulate the Interlaminar Stress Generated Near the Free Edge of a Composite Laminate, Delamination and Debonding of Materials, ASTM STP 876, American Society for Testing and Materials, pp. 35-51.

Cowper, G. R., (1966) The Shear Coefficient in Timoshenko's Beam Theory, Journal of Applied Mechanics, Vol. 33, pp. 335-340. 
Daniel, I. M., Hamilton, W. G. and Labedz, R. H. (1982) Strain Rate Characterization of Unidirectional Graphite/Epoxy Composite, Composite Materials: Testing and Design (Sixth Conference), ASTM STP 787, American Society for Testing and Materials, pp. 393-413.

Daniel, I. M., Ishai, O. (1994) Engineering Mechanics of Composite Materials, Oxford University Press, New York, USA.

Dickson, T., Munro, M. and Lee, S., (1995) Selection of an In-Plane Shear Test Method Based on the Shear Sensitivity of Laminate Tensile Modulus, Composite, Vol. 26, pp. 1724.

Feldman, A., Tasi, J. and Stang, D. A., (1966) Experimental Determination of Stiffness Properties of Thin-Shell Composite Cylinders, Experimental Mechanics, Vol. 6, pp. 385394.

Garcia, R., Weisshaar, T .A. and McWithey, R. R. (1980) An Experimental and Analytical Investigation of the Rail Shear-test Method as Applied to Composite Materials, Experimental Mechanics, Vol. 20, pp. 273-279.

Gere, J. M., (1963) Moment Distribution, D. Van Nostrand Co., Inc., Princeton, New Jersey, USA.

Ghali, A. and Neville, A. M., (1978) Structural Analysis, Richard Clay Ltd., Bungay, UK.

Gibson, R. F., (1994) Principles Composite Material Mechanics, McGraw-Hill, Inc., New York, USA. 
Gipple, K. L. and Hoyns, D., (1994) Measurement of The Out-of-Plane Shear Response of Thick Section Composite Materials Using the V-Notched Beam Specimen, J. Composite Materials, Vol. 28, pp. 543-572.

Goldsmith, W., (1960) Impact, the Theory and Physical Behavior of Colliding Solids, Edward Arnold, Ltd., London, England.

Gruber, B. M., Overbeeke, J. L. and Chou, T., (1982) A Reusable Sandwich Beam Concept for Composite Compression Test, J. Composite Materials, Vol. 16, pp. 162-171.

Hahn, H. T., (1973) A Note on Determination of the Shear Stress-Strain Response of Unidirectional Composites, J. Composite Materials, Vol. 7, pp. 383-386.

Herakovich, C. T., (1981) On the Relationship Between Engineering Properties and Delamination of Composite Materials, J. Composite Materials, Vol. 15, pp. 336-348.

Herakovich, C. T. and Bergner, H. W., (1980) Finite Element Stress Analysis of a Notched Coupon Specimen for In-Plane Shear Behavior of Composites, Composites, pp. 149-154.

Hofer, K. E. and Rao, P. N., (1977) A New Static Compression Fixture for Advanced Composite Materials, Journal of Testing and Evaluation, Vol. 5, pp. 278-283.

Iosipescu, N., (1967) New Accurate Procedure for Single Shear Testing of Metals, J. Materials, Vol. 2, pp. 537-566.

Isakson, G. and Levy, A., (1971) Finite-Element Analysis of Interlaminar Shear in Fibrous Composites, J. Composite Materials, Vol. 5, pp. 273-276. 
Jalali, S. J. and Taheri, F., (1998a) An Analytical Solution for Cross-Ply Laminated Plates Under Cylindrical Bending Based on Through-the-Thickness Inextensibility, Part I- Static loading, Int. J. Solids and Structures. Vol. 35, No. 14, pp. 1559-1574.

Jalali, S. J. and Taheri, F., (1998b) An Analytical Solution for Cross-Ply Laminated Plates under Cylindrical Bending Based on Through-the-Thickness Inextensibility, Part IIVibrations, Int. J. Solids and Structures. Vol. 35, No. 14, pp. 1575-1587.

Jalali, S. J. and Taheri, F., (1998c) Application of an Effective Solution on General Laminated Plates Under Cylindrical Bending with Shear Coupling Effect, to be published, Int. J. Iranica Scientia.

Jalali, S. J. and Taheri, F., (1998d) A New Test Method for Measuring the Longitudinal and Shear Moduli of Fiber Reinforced Composites, Submitted for publication to J. Composite Materials.

Jalali, S. J. and Taheri, F., (1997) Evaluation of the Elastic Properties of Fiber Reinforced Composites by the Varying-Span Method, Proceeding of Eleventh International Conference on Composite Materials (ICCM-11), Gold Coast, Australia, Vol. 5, pp. 625634.

Jones, A. T. (1970), Exact Natural Frequencies for Cross-Ply Laminates, J. Composite Materials, Vol. 4, pp. 476-491.

Kellas, S., Morton, J. and Jackson, K. E., (1993) Damage and Failure Mechanisms in Scaled Angled-Ply Laminates, Fourth Composites Symposium on Fatigue and Fracture, ASTM STP 1156, American Society for Testing and Materials, pp. 237-280. 
Knight, M. and Hahn, H. T., (1975) Strength and Elastic Modulus of a RandomlyDistributed Short Fiber Composite, J. Composite Materials, Vol. 9, pp. 77-89.

Lamothe, R. M. and Nunes, J., (1983) Evaluation of Fixturing for Compression Testing of Metal Matrix and Polymer/Epoxy Composites, Compression Testing of Homogeneous Materials and Composites, ASTM STP 808, American Society for Testing and Materials, pp. 241-253.

Lee, S. and Munro, M., (1986) Evaluation of In-Plane Shear Test Methods for Advanced Composite Materials by the Decision Analysis Technique, Composites, Vol. 17, pp. 13-22.

Lee, S., Munro, M. and Scott, R. F., (1990) Evaluation of Three In-Plane Shear Test Methods for Advanced Composite Materials, Composites, Vol. 21 pp. 495-502.

Lekhnitskii, S. G. (1981) Theory of Elasticity of an Anisotropic Body, Mir Publisher, Moscow, Russia.

Lo, K. H., Christensen, R. M. and Wu, E. M., (1977) A Higher-Order Theory of Plate Deformation, Part 2: Laminated Plates, J. Applied Mechanics, pp. 669-676.

Lockwood, P. A., (1981) Results of ASTM Round-Robin on the Rail Shear Test for Composites, Composites Technology Review, Vol. 3, pp. 83-93.

Mallick, P. K., (1993) Fiber-Reinforced Composites, Materials, Manufacturing, and Design, second edition, Marcel Dekker, Inc., New York, USA. 
Mindlin, R. D., (1951) Influence of Rotatory Inertia and Shear on Flexural Motions of Isotropic Elastic Plates, J. Applied Mechanics, Vol. 18, pp. 31-38.

Morton, J., Ho, H., Tsai, M. Y. and Earley, G. L., (1992) An Evaluation of the Iosipescu Specimen for Composite Materials Shear Property Measurement, J. Composite Materials, Vol. 26, pp. 708-750.

Munjal, A. K., (1989) Test Methods for Determining Design Allowables for Fiber Reinforced Composites, Test Methods for Design Allowables for Fiber Composites, ASTM STP 1003, American Society for Testing and Materials, pp. 93-110.

Munjal, A. K., Kulkarni, S. B., and Starrett, H. S., (1983) Characterization of Filament Wound Kevlar and Glass Composites for Rocket Motor Applications, 29th National SAMPE Symposium, pp. 324-337.

Pagano, N. J., (1969) Exact Solution for Composite Laminates in Cylindrical Bending, J. Composite material, Vol. 3, pp. 398-411.

Pagano, N. J. (1970), Influence of Shear Coupling in Cylindrical Bending of Anisotropic Laminates, J. Composite Materials, Vol. 4, pp. 330-343.

Pagano, N. J. and Whitney, J. M., (1970) Geometric Design of Composite Cylindrical Characterization Specimens, J. Composite Materials, Vol. 4, pp. 360-378.

Petit, P. H., (1969) A Simplified Method of Determining the In-Plane Shear Stress-Strain Response of Unidirectional Composites, Composite Material: Testing and Design, ASTM STP 460, American Society for Testing and Materials, pp. 83-93. 
Pierron, F., Vautrin, A. and Harris, B., (1995) The Iosipescu In-Plane Shear Test: Validation on an Isotropic Material, Experimental Mechanics, Vol. 35, pp. 130-135.

Piggott, M. R. and Harris, B., (1980) Compression Strength of Carbon, Glass and Kevlar49 Fiber Reinforced Polyester Resins, J. Materials Science, Vol. 15, pp. 2523-1538.

Pindera, M., Choksi, G. J., Hidde, S. and Herakovich, C. T., (1987) A Methodology for Accurate Shear Characterization of Unidirectional Composites, J. Composite Materials, Vol. 21, pp. 1164-1184.

Pindera, M. -J. and Herakovich, C. T., (1986) Shear Characterization of Unidirectional Composites with the Off-Axis Tension Test, Experimental Mechanics, Vol. 26, pp. 103112.

Pipes, R. B., Kaminski, B. E. and Pagano, N. J., (1973) Influence of the Free Edge upon the Strength of Angle-Ply Laminates, Analysis of the Test Method for High Modulus Fibers and Composites, ASTM STP 521, American Society for Testing and Materials, pp. 218-228.

Pipes, R. B. and Pagano, N. J., (1970) Interlaminar Stress in Composite Laminates Under Uniform Axial Extension, J. Composite Materials, Vol. 4, pp. 538-554.

Reddy, J. N., (1984) A Simple Higher-Order Theory for Laminated Composite Plates, J. Applied Mechanics Vol. 51, pp. 745-752.

Reddy, J. N., (1989) On Refined Computational Models of Composite Laminates, International Journal for Numerical Methods in Engineering, Vol. 27, pp. 361-382. 
Reissner, E., (1945) The Effect of Transverse Shear Deformation on the Bending of Elastic Plates, J. Applied Mechanics, Vol. 12, pp. A69-A77.

Reissner, E., (1975) On Transverse Bending of Plates, Including the Effect of Transverse Shear Deformation, Int. J. Solids and Structures, Vol. 11, pp. 569-573.

Rizzo, R. R. and Vicario, A. A., (1972) A finite Element Analysis for Stress Distribution in Gripped Tubular Specimens, Composite Materials: Testing and Design (Second Conference), ASTM STP 497, American Society for Testing and Materials, pp. 68-88.

Robbins, Jr, H. D. and Reddy, J. N., (1993) Modeling of Thick Composites Using a Layerwise Laminate Theory, International Journal for Numerical Methods in Engineering Vol. 36, pp. 655-677.

Rosen, B. W., (1972) A Simple Procedure for Experimental Determination of the Longitudinal Shear Modulus of Unidirectional Composites, J. Composite Materials, Vol. 6, pp. 552-554.

Rybicki, E. F., (1971) Approximate Three-Dimensional Solution for Symmetric Laminates Under Inplane Loading, J. composite Materials, Vol. 5, pp. 354-360.

Sandorff, P. E., (1980) Saint-Venant Effect in an Orthotropic Beam, J. Composite Materials, Vol. 14, pp. 199-211.

Sankar, B. V., (1989) Smooth Indentation of Orthotropic Beams, Composites Science and Technology, Vol. 34, pp. 95-112. 
Sattar, S. A. and Kellogg, D. H., (1969) The Effect of Geometry on the Mode of Failure of Composites in Short Beam Shear Test, Composite Material: Testing and Design, ASTM STP 460, American Society for Testing and Materials, pp. 62-71.

Schwartz, M. M., (1984) Composite Materials Handbook, McGraw-Hill Book Company, New York, USA.

Shokrieh, M. M., Eilers, O. P., Kotsiopriftis, P. and Lessard, L. B., (1995) Determination of Interlaminar Shear Strength of Graphite/Epoxy Composite Materials in Static and Fatigue Loading, Proceeding of ICCM-10, Whistler, B.C., Canada, Vol. 4, pp. 81-88.

Short, S. R. 1995. Characterization of Interlaminar Shear Failures of Graphite/Epoxy Composite Materials, Composites, Vol. 26, pp. 431-449.

Shuart, M. J., (1981) An Evaluation of the Sandwich Beam Compression Test Method for Composites, Test Method and Design Allowables for Fibrous Composites, ASTM STP 734, Ameriacn Society for Testing and Materials, pp. 152-165

Sims, D. F., (1973) In-Plane Shear Stress-Strain Response of Unidirectional Composite Materials, J. Composite Materials, Vol. 7, pp. 124-128.

Sinclair, J. H. and Chamis, C. C., (1983) Compressive Behavior of Unidirectional Fibrous Composites, ASTM STP 808, American Society for Testing and Materials, pp. 155-174.

Slepetz, J. M., Zageski, T. F., and Novello, R., (1978) In-Plane Shear Test for Composite Materials, Rep. AMMRC TR 78-30, Army Materials and Mechanics Research Center, Watertown, MA, USA. 
Smith, J. C., (1988) Structural Analysis, Harper \& Row, Publishers, Inc., New York, USA.

Soni, S. R. and Kim, R. Y., (1986) Delamination of Composite Laminates Stimulated by Interlaminar Shear, Composite Materials: Testing and Design, ASTM STP 893, American Society for Testing and Materials, pp. 286-307.

Spigel, B. S., Prabhakaran, R. and Sawyer, J. W., (1987) An Investigation of the Iosipescu and Asymmetrical Four-Point Bend Tests, Experimental Mechanics, Vol. 27, pp. 57-63.

Sullivan, J. L., Kao, B. G. and Van Oene, H., (1984) Shear Properties and a Stress Analysis Obtained from Vinyle-Ester Iosipescu Specimens, Experimental Mechanics, Vol. 24, pp. 223-232.

Sun, C. T. and Yamada, S., (1982) On the Measurement of Lamina In-Plane Shear Strength, Composites Technology Review, Vol. 4, pp. 52-53.

Swanson, S. R., Messick, M. and. Toombes, G. R., (1985) Comparison of Torsion Tube and Iosipescu In-Plane Shear Test Results for a Carbon Fibber-Reinforced Epoxy Composite, Composites, Vol. 16, pp. 220-224.

Terry, G., (1979) A Comparative Investigation of Some Method of Unidirectional, InPlane Shear Characterization of Composite Materials, Composites, Vol. 10, pp. 233-237.

Timoshenko, S. P., (1921) On The Correction for Shear of the Differential Equation for Transverse Vibrations of Prismatic Bars, Philosophical Magazine, Vol. 41, pp. 744-746. 
Timoshenko, S. P., (1922) On The Transverse Vibration of Bars of Uniform CrossSection, Philosophical Magazine, Vol. 43, pp. 125-131.

Timoshenko, S. P., (1983) History of Strength of materials, Dover Publications, Inc., New York, USA.

Timoshenko, S. P. and Goodier, J. N., (1970) Theory of Elasticity, 3rd edition, McGraw Hill Book Company, Inc., New York, USA.

Timoshenko, S. P., and Young, D. H., (1965) Theory of Structures, McGraw-Hill Book Company, New York, USA.

Tsai, C. L. and Daniel, I. M., (1990) Determination of In-Plane and Out-of-plane Shear Moduli of Composite Materials, Experimental Mechanics, Vol. 30, pp. 295-299.

Tsai, C. L., Daniel, I. M. and Yaniv, G., (1990) Torsional Response of Rectangular Composite Laminates, J. Applied Mechanics, Vol. 57, pp. 383-387.

Tsai, S. W. (1988) Composite Design, Fourth Edition, Think Composites, Dayton, USA.

Walrath, D. E. and Adams, D. F., (1983) The Iosipescu Shear Test as Applied to Composite Materials, Experimental Mechanics, Vol. 23, pp. 105-110.

Whitney, J. M., (1973) Free-Edge Effect in the Characterization of Composite Materials, Analysis of the Test Methods for High Modulus Fibers and Composites, ASTM STP 521, American Society for Testing and Materials, pp. 167-180. 
Whitney, J.M., (1985) Elasticity Analysis of Orthotropic Beams Under Concentrated Loads, Composites Science and technology, Vol. 22, pp. 167-184.

Whitney, J. M., (1987) Structural Analysis of Laminated Anisotropic Plates, Technomic Publishing Company, Inc., Lancaster, USA.

Whitney, J. M. and Browning, C. E. (1985) On Short-Beam Shear Tests for Composite Materials, Experimental Mechanics, Vol. 25, pp. 294-300.

Whitney, J. M., Browning C. E. and Mair, A., (1974) Analysis of the Flexural Test for Laminated Composite Materials, Composite Materials: Testing and Design, ASTM STP 546, American Society for Testing and Materials, pp. 30-45.

Whitney, J. M., Daniel, I. M. and Byron Pipes, R., (1984) Experimental Mechanics of Fiber Reinforced Composite Materials, Society for Experimental Mechanics, Brookfield Center, Conn., USA.

Whitney J. M. and Halpin, J. C., (1968) Analysis of Laminated Anisotropic Tubes Under Combined Loading, J. Composite Materials, Vol. 2, pp. 360-367.

Whitney, J. M. and Knight, M., (1980) The Relationship Between Tensile Strength and Flexure Strength in Fiber-Reinforced Composites, Experimental Mechanics, Vol. 20, pp. 211-216.

Whitney, J. M. and Pagano, N. J., (1970) Shear Deformation in Heterogeneous Anisotropic Plates, J. Applied Mechanics, pp. 1031-1036. 
Whitney, J. M. and Sun, C. T., (1973) A Higher Order Theory for Extentional Motion of Laminated Composites, J. Sound and Vibration, Vol. 30, pp. 85-97.

Whitney, J. M., Stansbarger, D. L. and Howell, H. B., (1971) Analysis of the Rail Shear Test-Applications and Limitations, J. Composite Materials, Vol. 5, PP. 24-34.

Xie, M. and Adams, D. F., (1995) Study of Three- and Four-Point Shear Testing of Unidirectional Composite Materials, Composites, Vol. 26, pp. 653-659.

Yang, P. C., Norris, C. H. and Stavsky, Y., (1966) Elastic Wave Propagation in Heterogeneous Plates, Int. J. Solids and Structures, Vol. 2, pp. 665-684.

Yeow, Y. T. and Brinson, H. F., (1978) A Comparison of Simple Shear Characterization Methods for Composite Laminates, Composites, Vol. 9, pp. 49-55.

Zhang, Y. and Sikarskie, D. L., (1996) On a Technique for Generating Stress-Strain Curves from Flexural Data, J. Composite Materials, Vol. 30, pp. 966-983.

Zweben, C., Smith, W. S. and Wardle, M. W., (1979) Test Methods for Fiber Tensile Strength, Composite Flexural Modulus and Properties of Fabric-Reinforced Laminates, Composite Materials: Testing and Design, ASTM STP 674, American Society for Testing and Materials, pp. 228-262. 


\section{Appendix A}

\section{ELAS-3P PROGRAM}

'The net, semi-net and total mid-span deflections of orthotropic beam under three-point 'bending is predicted by elasiticity solution

DECLARE SUB SOLVE (N!, AO AS DOUBLE, BO AS DOUBLE)

'LT: TOTAL LENGTH OF THE BEAM

'L: SPAN LENGTH

'H: THICKNESS OF THE BEAM

'F: CONCENTRATED LOAD AT MID-SPAN

'NSERI: NUMBER OF FOUREIR SERIES COMPONENTS IN THE ANALYSIS

'EX: MODULUS OF ELASTICITY IN X DIRECTION

'EZ: MODULUS OF ELASTICITY IN Y DIRECTION

'NUXZ: POISSON'S RATIO

'GXZ: THROUGH-THE-THICKNESS SHEAR MODULUS

'RM: THE RADIUS OF LOADING NOSE

'RS: THE RADIUS OF SUPPORTING ROLLERS

'ER: MODULUS OF ELASTICITY OF THE ROLLERS AND THE LOADING NOSE

'SDYNAMIC

DEFDBL $P$

CLS

READ LT, L, $H, F, N S E R I$

READ EX, EZ, NUXZ, GXZ

READ rm, rs, ER

DATA $16,8,2,1,100$

DATA $200,4,25,4$

DATA $5,5,200$

$\mathrm{PI}=4 \cdot \mathrm{ATN}(1)$

$D E L 1=1 /(E Z * P I)$

DEL2 $=1 /(E R * P I)$

BBAR $=2 * \operatorname{SQR}(F \cdot(D E L 1+D E L 2) * \mathrm{rm}) \quad$ CONTACT WIDTH AT MID-SPAN

CBAR $=2 * \operatorname{SQR}(F / 2 *(D E L 1+D E L 2) *$ rS $)$ 'CONTACT WIDTH AT THE SUPPORTS

$R 11=1 / E X$

$R 33=1 / E Z$

$R 13=-N U X Z / E X$

$R 66=1 / G X Z$

$A A=R 66+2 * R 13$

$B B=S Q R\left(A A \wedge 2 \cdot 4^{*} R 11 * R 33\right)$

$C C=2 * R 11$

DIM COEFMAT $(4,4)$ AS DOUBLE, A(4) AS DOUBLE

FOR $\|=1$ TO NSERI

$N=2 * \|-1$

$P=N \cdot P \mid / L T$

$M(1)=P \cdot S Q R((A A+B B) / C C)$

$M(2)=P \cdot S Q R((A A-B B) / C C)$

$M(3)=\cdot P * \operatorname{SQR}((A A+B B) / C C)$ 
$M(4)=-P * \operatorname{SQR}((A A-B B) / C C)$

'UNKNOWN COEFFICIENTS ARE DETRMINED IN THIS PART 'BY SATISFYING THE BOUNDARY CONDITIONS AT THE TOP 'AND THE BOTTOM OF THE BEAM
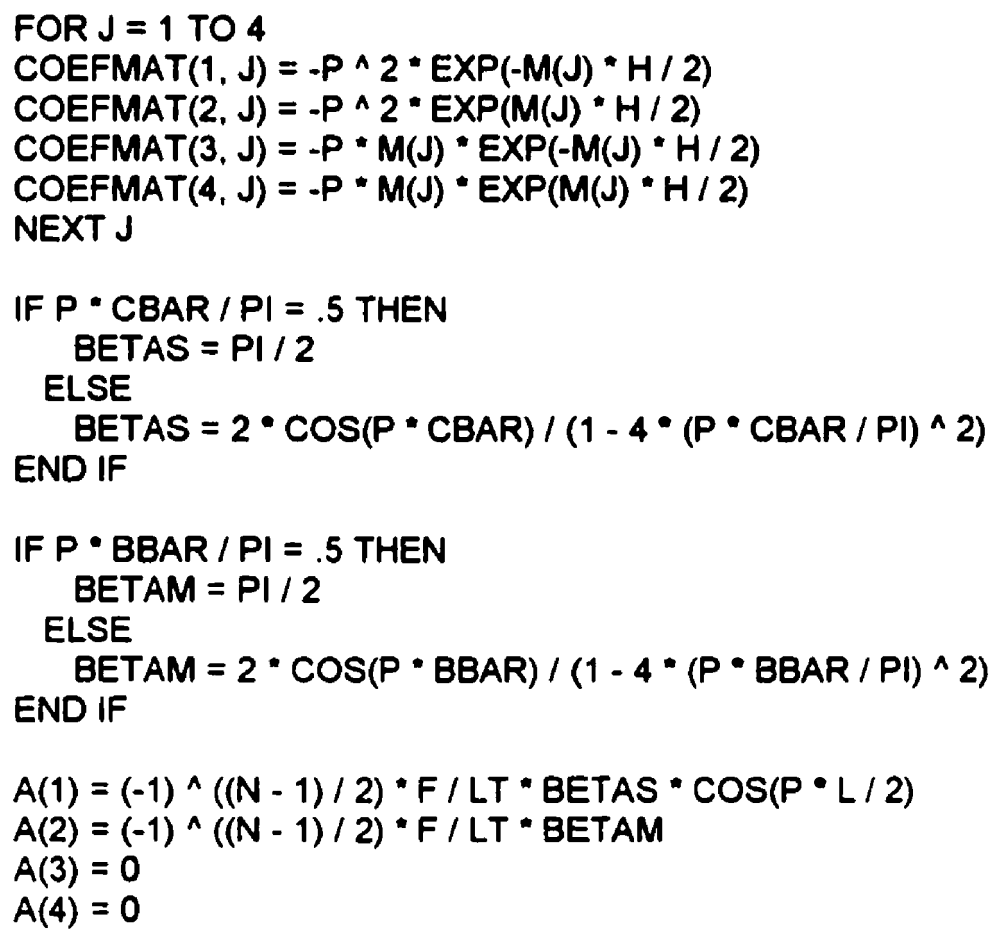

CALL SOLVE(4, COEFMATO, AO)

'Determination of Deflections

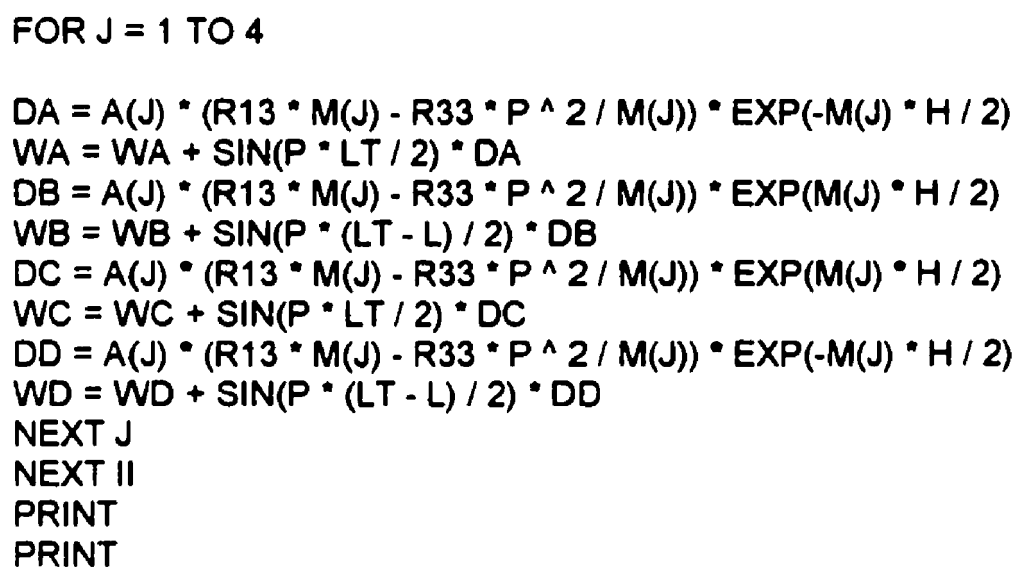

PRINT TAB(8): "WC-WD="; USING " \#\#\#伊 PRINT TAB(8); "WA-WD="; USING " "WA "WAAn"; WA - WD PRINT TAB(8); "WA-WB="; USING " W 


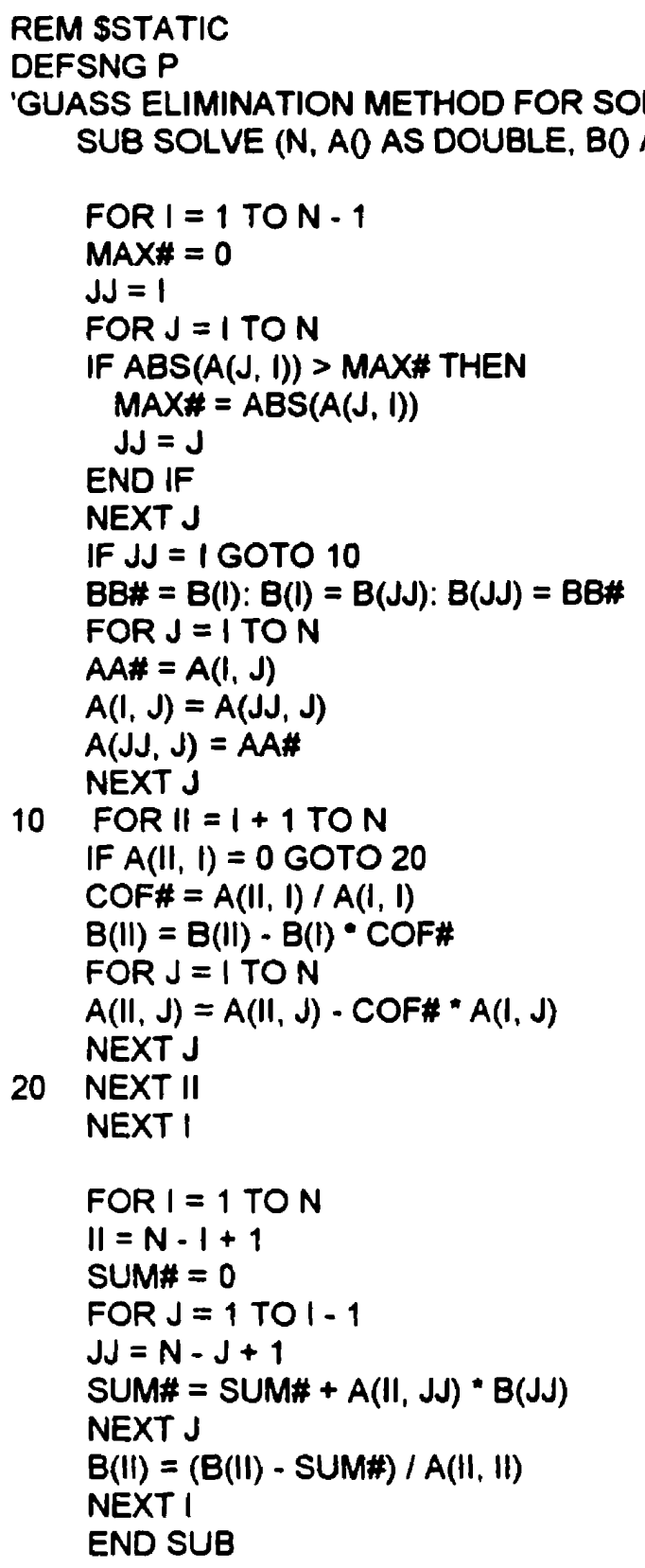




\section{Appendix B}

\section{ELAS-SIN PROGRAM}

'The behavior of simply supported orthotropic beam under HALF-SINE 'load is predicted by elasiticity solution

DECLARE SUB SOLVE (N!, AO AS DOUBLE, BO AS DOUBLE)

'L: SPAN LENGTH

'H: THICKNESS OF THE BEAM

'EX: MODULUS OF ELASTICITY IN X DIRECTION

'EZ: MODULUS OF ELASTICITY IN Y DIRECTION

'NUXZ: POISSON'S RATIO

'GXZ: THROUGH-THE-THICKNESS SHEAR MODULUS

'Note: The amplitude of the SINE load is 1.

' The load is applied on the top of the beam.

'SDYNAMIC

DEFDBL $P$

CLS

OPEN "ELAS-SIN.OUT" FOR OUTPUT AS \#1 'Open Output file

'Data Input

READ L, H, EX, EZ, NUXZ, GXZ

DATA $8,2,200,10,25,4$

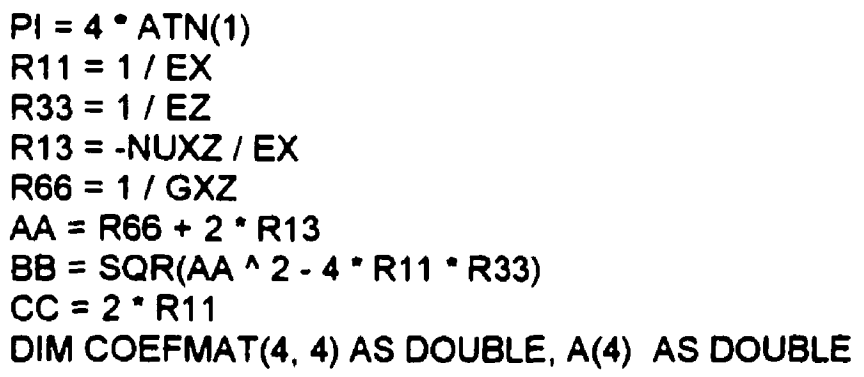

$$
\begin{aligned}
& P=P I / L \\
& M(1)=P * \operatorname{SQR}((A A+B B) / C C) \\
& M(2)=P * \operatorname{SQR}((A A-B B) / C C) \\
& M(3)=-P \cdot \operatorname{SQR}((A A+B B) / C C) \\
& M(4)=-P * \operatorname{SQR}((A A-B B) / C C)
\end{aligned}
$$

1.

'UNKNOWN COEFFICIENTS ARE DETRMINED IN THIS PART 'BY SATISFYING THE BOUNDARY CONDITIONS AT THE TOP 'AND THE BOTTOM OF THE BEAM

'Coefficients of the simultaneous equations 
FOR $\mathrm{J}=1$ TO 4

$\operatorname{COEFMAT}(1, J)=-P^{\wedge} 2 * \operatorname{EXP}(-M(J) * H / 2)$

$\operatorname{COEFMAT}(2, J)=-P \wedge 2 * \operatorname{EXP}(M(J) \bullet H / 2)$

$\operatorname{COEFMAT}(3, J)=-P * M(J) * \operatorname{EXP}(-M(J) * H / 2)$

$\operatorname{COEFMAT}(4, J)=-P * M(J) * \operatorname{EXP}(M(J) * H / 2)$

NEXT J

'KNOWN VECTORE

$A(1)=0$

$A(2)=1$

$A(3)=0$

$A(4)=0$

CALL SOLVE(4, COEFMATO, AO)

'Determination of Deflections and stresses

FOR $J=1$ TO 4

'Average deflection

COF $=A(J) *\left(R 13 * M(J)-R 33^{*} P \wedge 2 / M(J)\right) / M(J)$

COF $=\operatorname{COF}^{*}(\operatorname{EXP}(M(J) * H / 2)-\operatorname{EXP}(-M(J) * H / 2)) / H$

$W A=W A+C O F$

NEXT J

PRINT TAB(8); "Average Deflection:"; WA

PRINT

PRINT TAB(8); " ZH Deflection Sigxx";

PRINT " Sigzz TuXz"

PRINT \#1, TAB(8); "Average Deflection:"; WA

PRINT \#1,

PRINT \#1, TAB(8); " Z/H Deflection Sigxx";

PRINT \#1, " Sigzz Tuxz"

'Stresses at 11 points through the thickness

FOR I $=0$ TO 10

$\mathrm{DFL}=0$

COFXX $=0$

$\operatorname{COFZZ}=0$

COFXZ $=0$

$Z=I * H / 10 \cdot H / 2$

FOR $J=1$ TO 4

$D F L=D F L+A(J) *\left(R 13 * M(J) \cdot R 33^{*} P^{\wedge} 2 / M(J)\right) * \operatorname{EXP}(M(J) * Z)$

COFXX $=$ COFXX + A(J)*M(J)^ ${ }^{*} \cdot \operatorname{EXP}(M(J) * Z)$

COFZZ $=$ COFZZ + A(J)*EXP(M(J)*Z)

$\operatorname{COFXZ}=\operatorname{COFXZ}+A(J) * M(J) * \operatorname{EXP}(M(J) * Z)$

NEXT J

DEFLECTION = DFL 
$\operatorname{SIGXX}=\operatorname{COFXX}$

Sigzz $=-P^{\wedge} 2 \cdot \operatorname{COFZZ}$

TuXz $=-P * \operatorname{COFXZ}$

PRINT TAB(8); USING "\#.\#"; $Z$ / H;

PRINT USING " \#.\#\#^^^"; DEFLECTION; SIGXX; Sigzz; TUXZ

PRINT \#1, TAB(8); USING "\#.\#; $Z / H$;

PRINT \#1, USING " \#.\#\#^^^n“; DEFLECTION; SIGXX; Sigzz; TUXZ

NEXT I

REM \$STATIC

DEFSNG $P$

'GUASS ELIMINATION METHOD FOR SOLVING S!A:IULTANEOUS EQUATIONS SUB SOLVE (N, AO AS DOUBLE, BO AS DOUBLE)

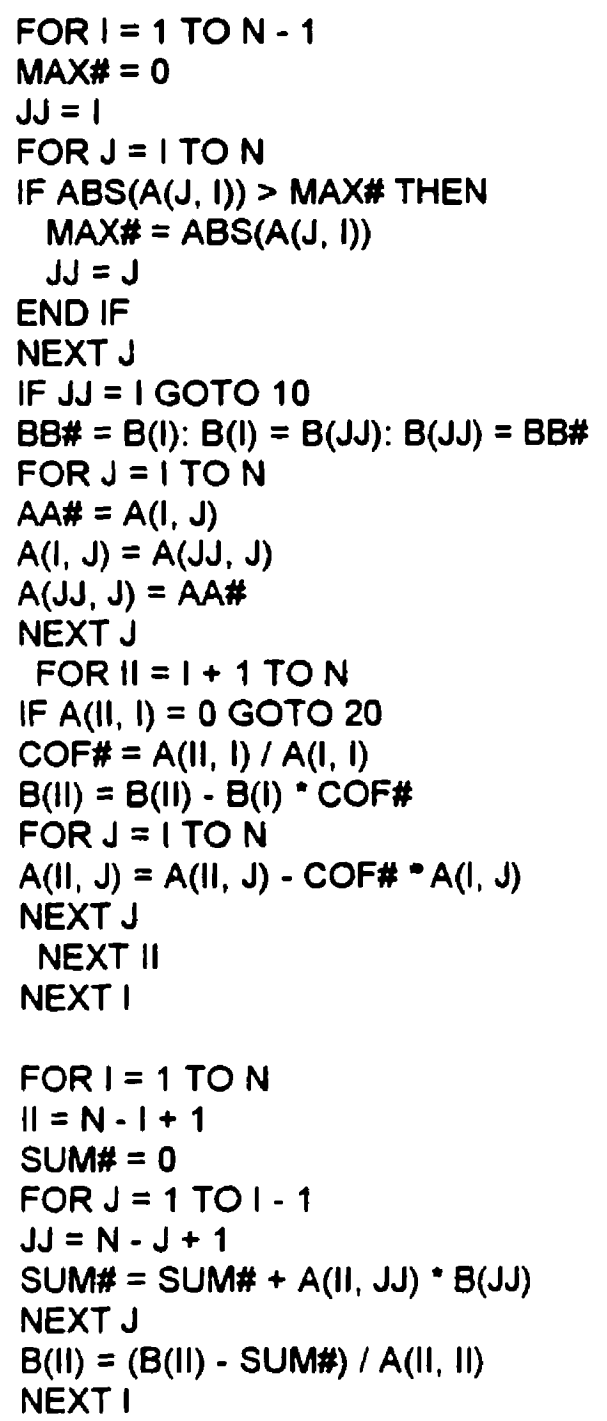

END SUB 


\section{Appendix C}

\section{TTTI-SIN PROGRAM}

'The behavior of simply supported orthotropic beam under HALF-SINE load 'is determined by the application of the through-the-thickness inextensibility 'theory.

\section{DECLARE FUNCTION SINH\# (X AS DOUBLE)}

DECLARE FUNCTION COSH\# (X AS DOUBLE)

DECLARE FUNCTION TANH\# (X AS DOUBLE)

'L: SPAN LENGTH

'H: THICKNESS OF THE BEAM

'EX: MODULUS OF ELASTICITY IN X DIRECTION

'GXZ: THROUGH-THE-THICKNESS SHEAR MODULUS

'Note: The amplitude of the SINE load is 1.

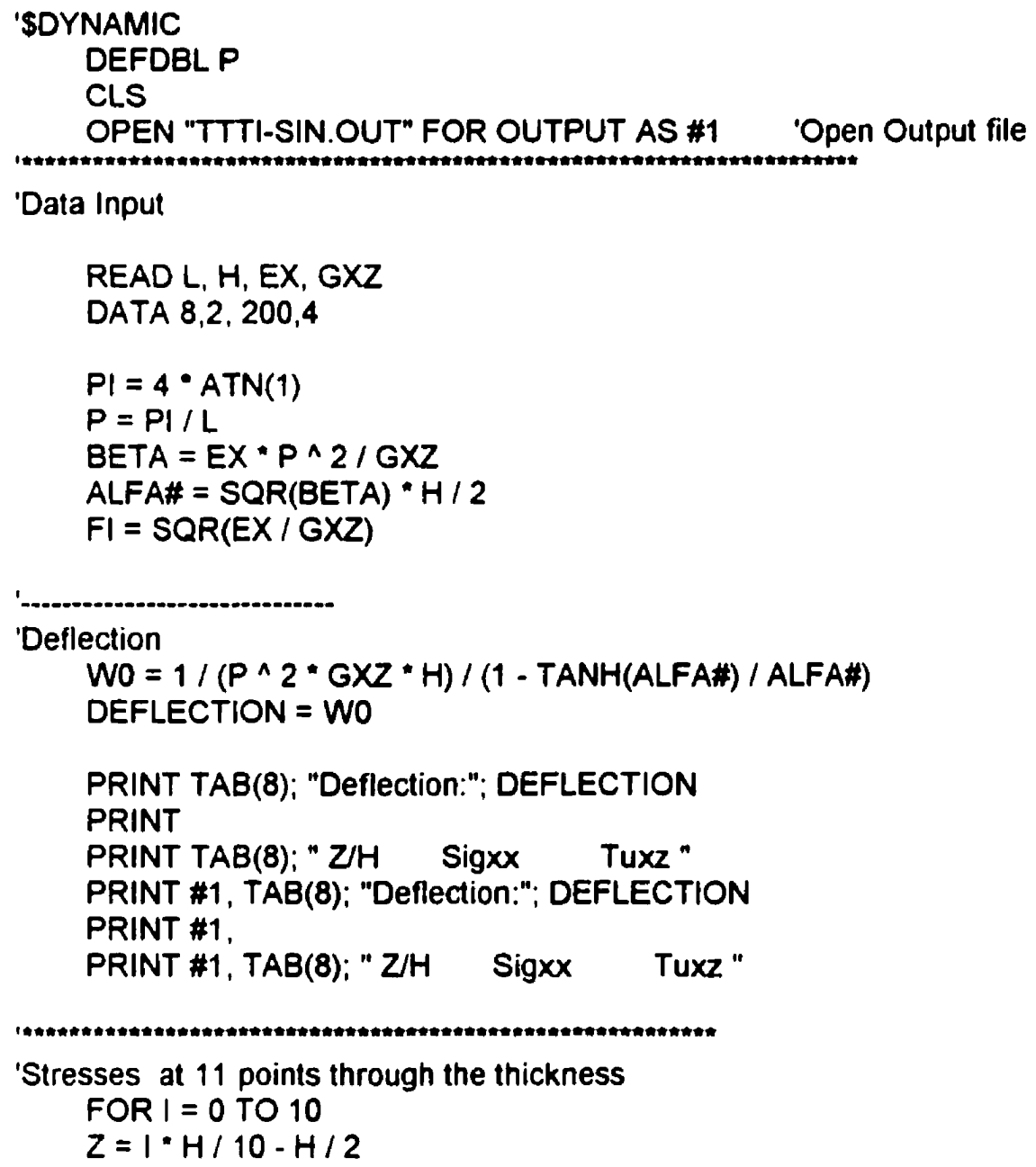

'Stresses at 11 points through the thickness

FOR I $=0$ TO 10

$Z=I * H / 10-H / 2$ 
'Sigmaxx

C1 $=F I^{\wedge} 2 /($ ALFA\# - TANH(ALFA\#)) $/ 2$

$C 2=\operatorname{SINH}(2 \cdot A L F A \# \cdot Z / H) / \operatorname{COSH}(A L F A \#)$

Sig $x \mathrm{x}=\mathrm{C}_{1} \cdot \mathrm{C}_{2}$

'Tuxz

$\mathrm{C} 1=\mathrm{FI} /($ (ALFA\# $-\mathrm{TANH}($ ALFA\#) $) / 2$

$\mathrm{C} 2=\left(1-\operatorname{COSH}\left(2^{-} \operatorname{ALFA\# }^{*} \mathrm{Z} / \mathrm{H}\right) / \mathrm{COSH}(\mathrm{ALFA \#})\right)$

TUXZ $=\mathrm{C}_{1}{ }^{\circ} \mathrm{C} 2$

PRINT TAB(8); USING "\#.\#"; $Z / H$;

PRINT USING " \#.\#

PRINT \#1, TAB(8); USING "\#\#.\#"; $Z / H$;

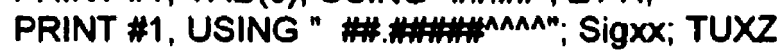

NEXT I

'Function to determine "cosine hyperbola" FUNCTION COSH\# (X AS DOUBLE)

$\mathrm{COSH}=.5 *(\operatorname{EXP}(\mathrm{X})+\operatorname{EXP}(-X))$

END FUNCTION

'Function to determine "sine hyperbola"

FUNCTION SINH\# (X AS DOUBLE)

$\operatorname{SINH}=.5^{*}(\operatorname{EXP}(X)-\operatorname{EXP}(-X))$

END FUNCTION

'Function to determine "tan hyperbola"

FUNCTION TANH\# (X AS DOUBLE)

TANH $=\operatorname{SINH}(X) / \operatorname{COSH}(X)$

END FUNCTION 


\section{Appendix D}

\section{ELAS-CON PROGRAM}

'The behavior of simply supported orthotropic beam under a concentrated load 'at mid-span is determined according to the elasiticity solution.

DECLARE SUB SOLVE (N!, AO AS DOUBLE, BO AS DOUBLE)

'L: SPAN LENGTH

'H: THICKNESS OF THE BEAM

'F: CONCENTRATED LOAD AT MID-SPAN

'NSERI: NUMBER OF FOUREIR SERIES COMPONENTS IN THE ANALYSIS

'EX: MODULUS OF ELASTICITY IN X DIRECTION

'EZ: MODULUS OF ELASTICITY IN Y DIRECTION

'NUXZ: POISSON'S RATIO

'GXZ: THROUGH-THE-THICKNESS SHEAR MODULUS

'R: THE RADIUS OF LOADING NOSE

'ES: MODULUS OF ELASTICITY OF THE ROLLERS AND THE LOADING NOSE

' $X$ : COORDINATE OF THE SECTION THAT THE CALCULATION MUST BE DONE FOR

'SDYNAMIC

DEFDBL $P$

OPEN "ELAS-CON.OUT" FOR OUTPUT AS \#1

CLS

READ L, X, H, F, NSERI

READ EX, EZ, NUXZ, GXZ

READ R, ES

DATA $8,4,2,1,200$

DATA $200,10, .25,4$

DATA 5,200

$\mathrm{PI}=4 * \mathrm{ATN}(1)$

DEL1 $=1 /(E Z-P I)$

DEL2 $=1 /(E S * P I)$

$B B A R=2$-SQR(F * (DEL1 + DEL2) *R) 'HALF OF CONTACT WIDTH

$\mathrm{R} 11=1 / \mathrm{EX}$

$R 33=1 / E Z$

$R 13=-N U X Z / E X$

$R 66=1 / G X Z$

$A A=R 66+2 * R 13$

$B B=S Q R(A A \wedge 2-4 * R 11 * R 33)$

$C C=2^{\circ}$ R11

DIM COEFMAT $(4,4)$ AS DOUBLE, A(4) AS DOUBLE

FOR $N=1$ TO NSERI STEP 2

$P=N * P I / L$

$M(1)=P \cdot \operatorname{SQR}((A A+B B) / C C)$

$M(2)=P \cdot \operatorname{SQR}((A A-B B) / C C)$ 
$M(3)=-P * S Q R((A A+B B) / C C)$

$M(4)=-P \cdot \operatorname{SQR}((A A-B B) / C C)$

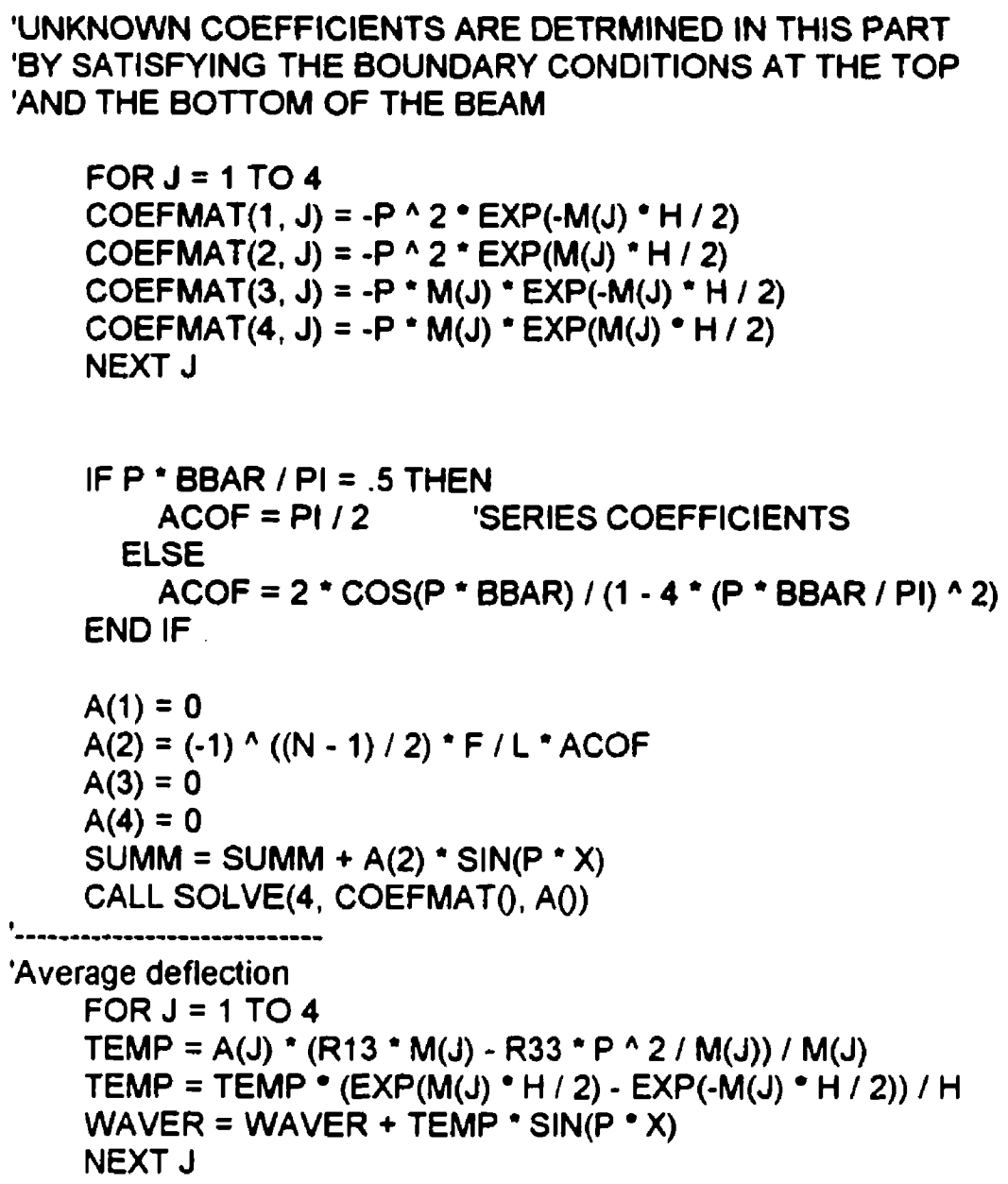

'Determination of Deflection AND STRESSES FOR 11 ELEVATIONS AT $x=x$

FOR $I=0$ TO 10

$Z=1 \cdot H / 10 \cdot H / 2$

FOR $\mathrm{J}=1 \mathrm{TO} 4$

$W 0=A(J) *(R 13 * M(J)-R 33 * P \wedge 2 / M(J)) * \operatorname{EXP}(M(J) * Z)$

$W(I)=W(I)+W O * \operatorname{SIN}(P \cdot X)$

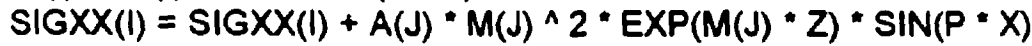

$\operatorname{SIGZZ}(I)=\operatorname{SIGZZ}(I)-P^{\wedge} 2^{*} A(J) * \operatorname{EXP}(M(J) * Z) * \operatorname{SIN}(P * X)$

$\operatorname{TUXZ}(I)=\operatorname{TUXZ}(I)-P * A(J) * M(J) * \operatorname{EXP}(M(J) * Z) * \operatorname{COS}(P * X)$

NEXT J

NEXT I

NEXT N 
'PRINT OUTPUT

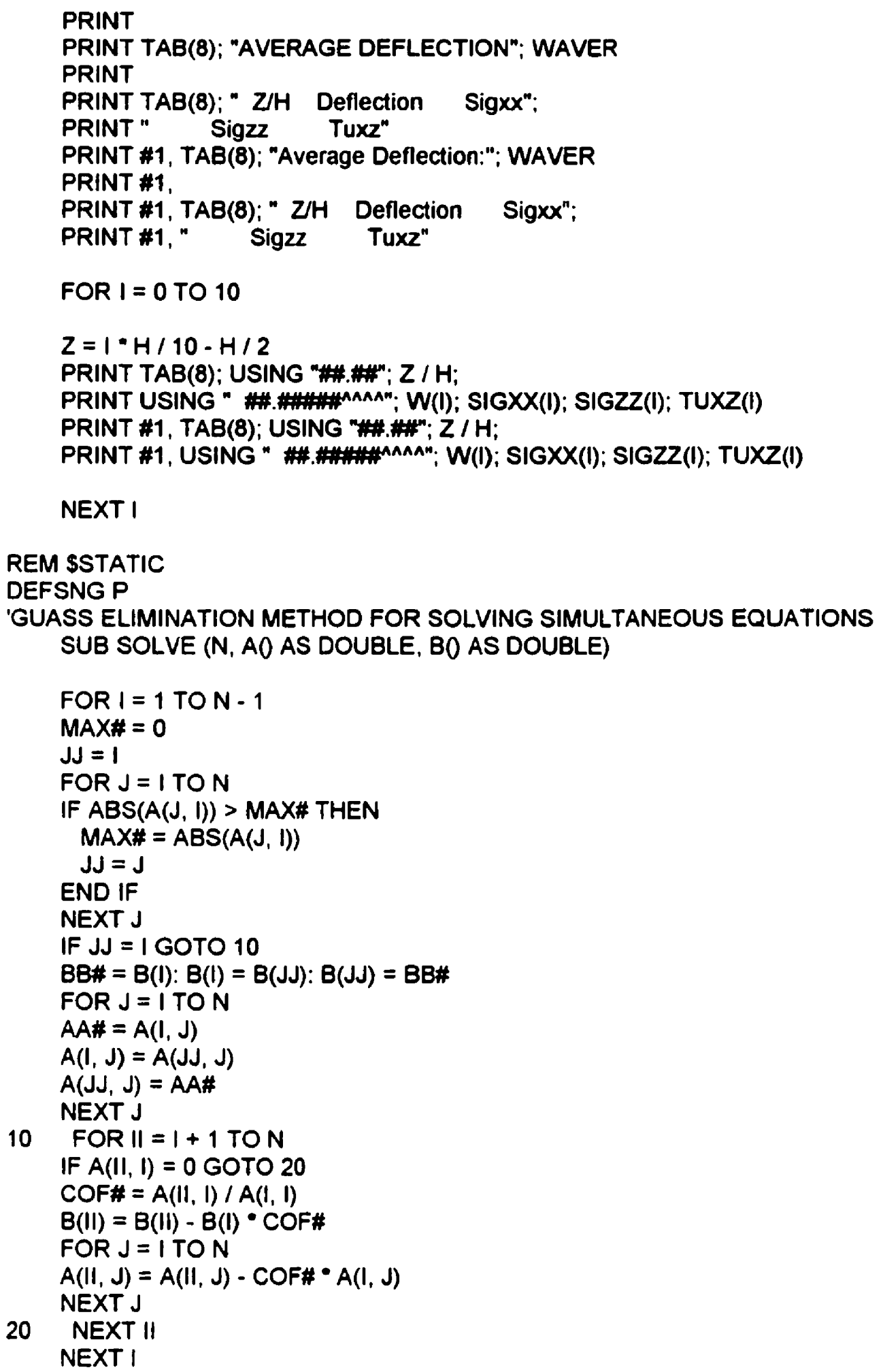




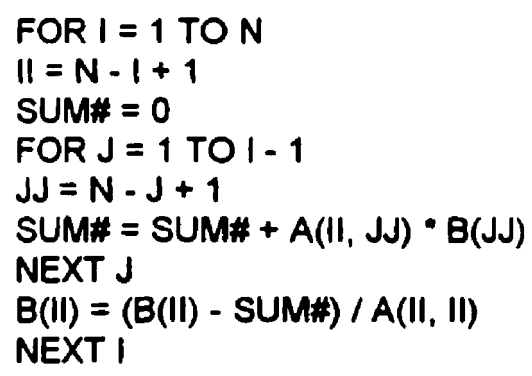

$B(I I)=(B(I I)-$ SUM\#) / A(II, II) NEXT I

END SUB 


\section{Appendix E \\ TTTI-CON PROGRAM}

'The behavior of simply supported orthotropic beam under a concentrated load 'at mid-span is determined by the application of the through-the-thickness "inextensibility theory.

DECLARE SUB SERIESCOFF (LOADDIST!, P\#, BBAR!, ACOF!)

DECLARE SUB SERICOFF (LOADDIST!, P\#, BBAR!, ACOF!)

DECLARE SUB HERTZIAN (P\#, BBAR!, ACOF!)

DECLARE FUNCTION SINH\# (X AS DOUBLE)

DECLARE FUNCTION COSH\# (X AS DOUBLE)

DECLARE FUNCTION TANH\# (X AS DOUBLE)

'The distribution of the concentrated load on the beam may follow 'one of the following options:

'1- Uniform; LOADDIST=1

'2- Hertezian contact law; LOADDIST $=2$

'3- Eqn (4.31); LOADDIST $=3$

'L: SPAN LENGTH

'H: THICKNESS OF THE BEAM

' $X$ : THE COORDINATE OF THE POINT THAT THE ANALYSIS IS DONE FOR

'EX: MODULUS OF ELASTICITY OF THE BEAM IN X DIRECTION

'EZ: MODULUS OF ELASTICITY OF THE BEAM IN Z DIRECTION

'ES: MODULUS OF ELASTICITY OF THE LOADING NOSE

'GXZ: THROUGH-THE-THICKNESS SHEAR MODULUS

'F: MAGNITUDE OF THE CONCENTRATED LOAD

'R: RADIUS OF THE LOADING NOSE

'NSERIES: NUMBER OF THE SERIES COMPONENTS TO BE CONSIDERED

'BBARRATIO: HALF CONTACT LENGTH TO THICKNESS RATIO. IS NOT USED FOR LOADDIST $=2$

'SOYNAMIC

DEFDBL $P$

CLS

OPEN "TTTI-CON.OUT" FOR OUTPUT AS \#1 'Open Output file

'Data Input

LOADDIST $=3$

READ L, X, H, EX, GXZ

DATA $8,2,2,200,4$

READ F, EZ, ES, R, NSERIES

DATA . 1, 10,200,5,200

READ BBARRATIO 'FOR LOADDIST=1 AND 3

DATA 1.45 
BBAR $=H \cdot B B A R R A T I O$

$\mathrm{PI}=4^{\circ} \mathrm{ATN}(1)$

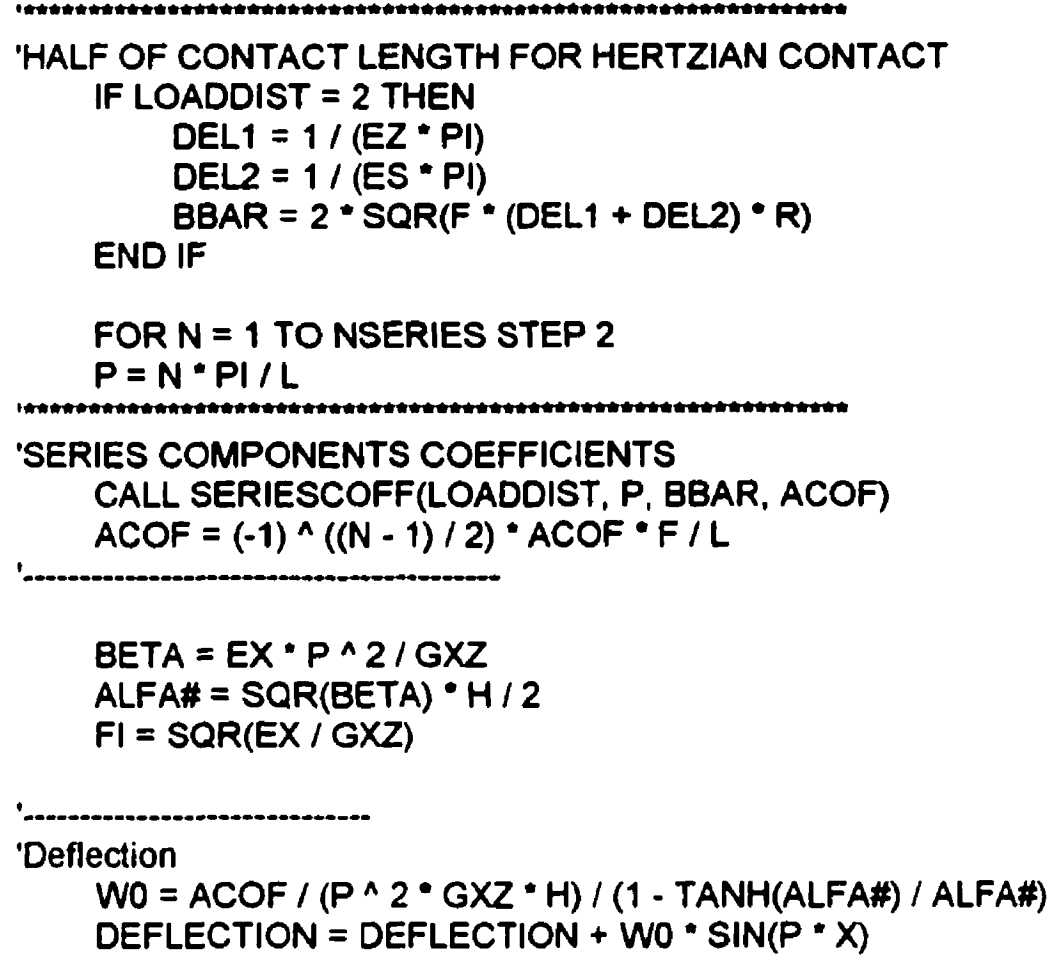

'Stresses at 11 points through the thickness

FOR I $=0$ TO 10

$Z=I * H / 10-H / 2$

'Sigmaxx

$\mathrm{C} 1=\mathrm{FI} \wedge 2 /$ (ALFA\# $-\mathrm{TANH}($ ALFA\#)) $/ 2$

$C 2=\operatorname{SINH}(2 * A L F A \# \cdot Z / H) / C O S H(A L F A \#)$

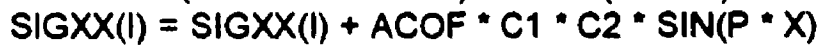

'Tuxz

$C 1=F I /($ ALFA\# - TANH(ALFA\#) $/ 2$

$\mathrm{C} 2=(1-\operatorname{COSH}(2 * A L F A \# * Z / H) / \operatorname{COSH}(A L F A \#))$

$\operatorname{TUXZ}(I)=\operatorname{TUXZ}(I)+A C O F * C_{1} * C_{2} * \operatorname{COS}(P \cdot X)$

NEXT I

NEXT N

PRINT TAB(8); "Deflection:"; DEFLECTION

PRINT

PRINT TAB(8); " Z/H Sigxx Tuxz "

PRINT \#1, TAB(8); "Deflection:"; DEFLECTION 
PRINT \#1,

PRINT \#1, TAB(8); " Z/H Sigxx Tuxz "

FOR I $=0$ TO 10

$Z=1 * H / 10-H / 2$

PRINT TAB(8); USING \#.\#; $Z / H$;

PRINT USING " \#A^^^"; SIGXX(1): TUXZ(I)

PRINT \#1, TAB(8); USING "\#.\#; $Z / H$;

PRINT \#1, USING " \#.\#AnAn"; SIGXX(I); TUXZ(1)

NEXT I

'Function to determine "cosine hyperpola

FUNCTION COSH\# (X AS DOUBLE)

$\mathrm{COSH}=.5 *(\operatorname{EXP}(\mathrm{X})+\operatorname{EXP}(-\mathrm{X}))$

END FUNCTION

REM SSTATIC

'Subroutine to determine the coefficinets of Fourier series

SUB SERIESCOFF (LOADDIST, P, BBAR, ACOF)

SHARED PI

IF LOADDIST $=1$ THEN

$A C O F=2 * \operatorname{SIN}(P * B B A R) /(P * B B A R)$

END IF

IF LOADDIST $=2$ THEN

IF P • BBAR / PI $=.5$ THEN

$A C O F=P I / 2$

ELSE

$A C O F=2 * \operatorname{COS}(P * B B A R) /\left(1-4 *(P * B B A R / P I)^{\wedge} 2\right)$

END IF

END IF

IF LOADDIST $=3$ THEN

$C C=\operatorname{SIN}(P \cdot B B A R)$

$C 1=C C / P$

IF $P \cdot B B A R=4^{\circ} \mathrm{PI}$ THEN

$C 2=B B A R / 2$

ELSE

$C 2=-\left(P * B B A R \wedge 2 /\left(16 * P I^{\wedge} 2\right) * C C /\left(1 \cdot\left(P * B B A R /\left(4^{*} P I\right)\right)^{\wedge} 2\right)\right)$

END IF

IF BP * BBAR $=3 * \mathrm{PI}$ THEN

$C 3=B B A R / 2$

ELSE

$C 3=\left(P \bullet B B A R \wedge 2 /\left(9^{\bullet} P I^{\wedge} 2\right){ }^{\star} C C /\left(1-\left(P^{\bullet} B B A R /\left(3^{\bullet} P I\right)\right)^{\wedge} 2\right)\right)$

END IF

IF $P \cdot B B A R=2 * P I$ THEN

$C 4=B B A R / 2$

ELSE

$C 4=-\left(P * B B A R \wedge 2 /\left(4^{*} P I^{\wedge} 2\right) * C C /\left(1-(P * B B A R /(2 * P I))^{\wedge} 2\right)\right)$

END IF

IF P * BBAR = PI THEN

$\mathrm{C} 5=\mathrm{BBAR} / 2$

ELSE 


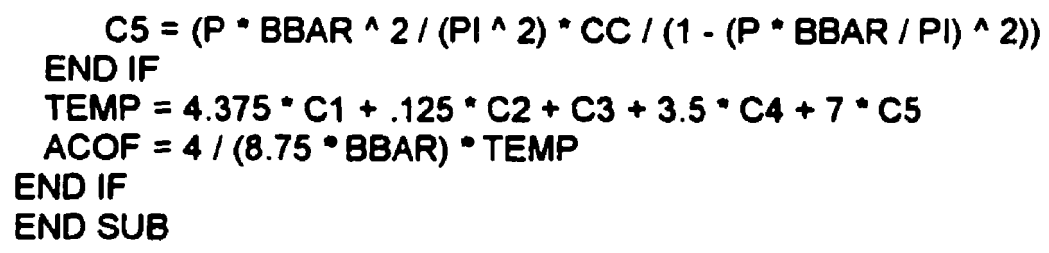

REM SDYNAMIC

'Function to determine "sine hyperbola" FUNCTION SINH\# (X AS DOUBLE)

$\operatorname{SINH}=.5^{\circ}(\operatorname{EXP}(X)-\operatorname{EXP}(-X))$

END FUNCTION

'Function to determine "tan hyperbola"

FUNCTION TANH\# (X AS DOUBLE)

TANH $=\operatorname{SINH}(X) / \operatorname{COSH}(X)$

END FUNCTION 


\section{Appendix F}

THE MATERIAL PROPERTIES USED FOR THE

DETERMINATION OF $\alpha$

Table F. 1 The material properties used for the determination

\begin{tabular}{cccccc} 
of $\alpha$ \\
$\begin{array}{c}\text { Maretial } \\
\text { ID }\end{array}$ & $E_{11}$ & $E_{22}, E_{33}$ & $\begin{array}{c}v_{12}, v_{13}, \\
V_{23}\end{array}$ & $G_{12}, G_{13}$ & $G_{23}$ \\
\hline AA0 & 200 & 4 & .25 & 4 & 2 \\
AA1 & 200 & 8 & .25 & 4 & 2 \\
AA2 & 200 & 12 & .25 & 4 & 2 \\
AA3 & 200 & 16 & .25 & 4 & 2 \\
AB0 & 200 & 5 & .25 & 5 & 2.5 \\
AB1 & 200 & 10 & .25 & 5 & 2.5 \\
AB2 & 200 & 15 & .25 & 5 & 2.5 \\
AB3 & 200 & 20 & .25 & 5 & 2.5 \\
AC0 & 200 & 6.667 & .25 & 6.667 & 3.333 \\
AC1 & 200 & 13.333 & .25 & 6.667 & 3.333 \\
AC2 & 200 & 20 & .25 & 6.667 & 3.333 \\
AC3 & 200 & 26.667 & .25 & 6.667 & 3.333 \\
AD0 & 200 & 10 & .25 & 10 & 5 \\
AD1 & 200 & 20 & .25 & 10 & 5 \\
AD2 & 200 & 30 & .25 & 10 & 5 \\
AD3 & 200 & 40 & .25 & 10 & 5 \\
AE0 & 200 & 20 & .25 & 20 & 10 \\
AE1 & 200 & 40 & .25 & 20 & 10 \\
AE2 & 200 & 60 & .25 & 20 & 10 \\
AE3 & 200 & 80 & .25 & 20 & 10 \\
BA0 & 200 & 4 & .3 & 4 & 2 \\
BA1 & 200 & 8 & .3 & 4 & 2 \\
BA2 & 200 & 12 & .3 & 4 & 2 \\
BA3 & 200 & 16 & .3 & 4 & 2 \\
BB0 & 200 & 5 & .3 & 5 & 2.5 \\
BB1 & 200 & 10 & .3 & 5 & 2.5 \\
BB2 & 200 & 15 & .3 & 5 & 2.5 \\
BB3 & 200 & 20 & .3 & 5 & 2.5 \\
BC0 & 200 & 6.667 & .3 & 6.667 & 3.333 \\
BC1 & 200 & 13.333 & .3 & 6.667 & 3.333 \\
BC2 & 200 & 20 & .3 & 6.667 & 3.333 \\
\hline & & & & &
\end{tabular}


Table F.1 (Continued)

\begin{tabular}{|c|c|c|c|c|c|}
\hline $\begin{array}{c}\text { Maretial } \\
\text { ID } \\
\end{array}$ & $E_{11}$ & $E_{22}, E_{33}$ & $\begin{array}{c}v_{12}, v_{13}, \\
v_{23}\end{array}$ & $G_{12}, G_{13}$ & $G_{23}$ \\
\hline $\mathrm{BC} 3$ & 200 & 26.667 & 3 & 6.667 & 3.333 \\
\hline BD0 & 200 & 10 & .3 & 10 & 5 \\
\hline BDI & 200 & 20 & .3 & 10 & 5 \\
\hline BD2 & 200 & 30 & .3 & 10 & 5 \\
\hline BD3 & 200 & 40 & .3 & 10 & 5 \\
\hline BE0 & 200 & 20 & .3 & 20 & 10 \\
\hline BEl & 200 & 40 & .3 & 20. & 10 \\
\hline BE2 & 200 & 60 & .3 & 20 & 10 \\
\hline BE3 & 200 & 80 & .3 & 20 & 10 \\
\hline $\mathrm{CA} 0$ & 200 & 4 & .35 & 4 & 2 \\
\hline CAl & 200 & 8 & .35 & 4 & 2 \\
\hline CA2 & 200 & 12 & .35 & 4 & 2 \\
\hline CA3 & 200 & 16 & .35 & 4 & 2 \\
\hline CB0 & 200 & 5 & .35 & 5 & 2.5 \\
\hline CBI & 200 & 10 & .35 & 5 & 2.5 \\
\hline CB2 & 200 & 15 & .35 & 5 & 2.5 \\
\hline CB3 & 200 & 20 & .35 & 5 & 2.5 \\
\hline $\mathrm{CC} 0$ & 200 & 6.6667 & .35 & 6.667 & 3.333 \\
\hline $\mathrm{CCl}$ & 200 & 13.333 & .35 & 6.667 & 3.333 \\
\hline $\mathrm{CC} 2$ & 200 & 20 & .35 & 6.667 & 3.333 \\
\hline $\mathrm{CC} 3$ & 200 & 26.667 & .35 & 6.667 & 3.333 \\
\hline CD0 & 200 & 10 & .35 & 10 & 5 \\
\hline CD1 & 200 & 20 & .35 & 10 & 5 \\
\hline CD2 & 200 & 30 & .35 & 10 & 5 \\
\hline CD3 & 200 & 40 & .35 & 10 & 5 \\
\hline CE0 & 200 & 20 & .35 & 20 & 10 \\
\hline CEl & 200 & 40 & .35 & 20 & 10 \\
\hline CE2 & 200 & 60 & .35 & 20 & 10 \\
\hline CE3 & 200 & 80 & .35 & 20 & 10 \\
\hline DA0 & 200 & 4 & .25 & 4 & 4 \\
\hline DAI & 200 & 8 & .25 & 4 & 4 \\
\hline DA2 & 200 & 12 & .25 & 4 & 4 \\
\hline DA3 & 200 & 16 & .25 & 4 & 4 \\
\hline DB0 & 200 & 5 & .25 & 5 & 5 \\
\hline DB 1 & 200 & 10 & .25 & 5 & 5 \\
\hline DB2 & 200 & 15 & .25 & 5 & 5 \\
\hline DB3 & 200 & 20 & .25 & 5 & 5 \\
\hline $\mathrm{DC} 0$ & 200 & 6.667 & .25 & 6.667 & 6.667 \\
\hline $\mathrm{DCl}$ & 200 & 13.333 & .25 & 6.667 & 6.667 \\
\hline
\end{tabular}


Table F.1 (Continued)

\begin{tabular}{|c|c|c|c|c|c|}
\hline $\begin{array}{l}\text { Maretial } \\
\text { ID }\end{array}$ & $E_{11}$ & $E_{22}, E_{33}$ & $\begin{array}{c}v_{12}, v_{13}, \\
v_{23}\end{array}$ & $G_{12}, G_{13}$ & $G_{23}$ \\
\hline DC2 & 200 & 20 & .25 & 6.667 & 6.6667 \\
\hline DC3 & 200 & 26.667 & .25 & 6.667 & 6.6667 \\
\hline DD0 & 200 & 10 & .25 & 10 & 10 \\
\hline DDl & 200 & 20 & .25 & 10 & 10 \\
\hline DD2 & 200 & 30 & .25 & 10 & 10 \\
\hline DD3 & 200 & 40 & .25 & 10 & 10 \\
\hline DE0 & 200 & 20 & .25 & 20 & 20 \\
\hline DEl & 200 & 40 & .25 & 20 & 20 \\
\hline DE2 & 200 & 60 & .25 & 20 & 20 \\
\hline DE3 & 200 & 80 & .25 & 20 & 20 \\
\hline EA0 & 200 & 4 & .3 & 4 & 4 \\
\hline EAl & 200 & 8 & .3 & 4 & 4 \\
\hline EA2 & 200 & 12 & .3 & 4 & 4 \\
\hline EA3 & 200 & 16 & 3 & 4 & 4 \\
\hline EB0 & 200 & 5 & .3 & 5 & 5 \\
\hline EBI & 200 & 10 & .3 & 5 & 5 \\
\hline EB2 & 200 & 15 & .3 & 5 & 5 \\
\hline EB3 & 200 & 20 & .3 & 5 & 5 \\
\hline EC0 & 200 & 6.667 & .3 & 6.667 & 6.6667 \\
\hline $\mathrm{ECl}$ & 200 & 13.333 & .3 & 6.667 & 6.6667 \\
\hline $\mathrm{EC} 2$ & 200 & 20 & .3 & 6.667 & 6.6667 \\
\hline $\mathrm{EC} 3$ & 200 & 26.667 & .3 & 6.667 & 6.6667 \\
\hline ED0 & 200 & 10 & 3 & 10 & 10 \\
\hline EDI & 200 & 20 & .3 & 10 & 10 \\
\hline ED2 & 200 & 30 & .3 & 10 & 10 \\
\hline ED3 & 200 & 40 & .3 & 10 & 10 \\
\hline EE0 & 200 & 20 & 3 & 20 & 20 \\
\hline EEl & 200 & 40 & 3 & 20 & 20 \\
\hline EE2 & 200 & 60 & 3 & 20 & 20 \\
\hline EE3 & 200 & 80 & .3 & 20 & 20 \\
\hline FA0 & 200 & 4 & .35 & 4 & 4 \\
\hline FAl & 200 & 8 & .35 & 4 & 4 \\
\hline FA2 & 200 & 12 & .35 & 4 & 4 \\
\hline FA3 & 200 & 16 & .35 & 4 & 4 \\
\hline FB0 & 200 & 5 & .35 & 5 & 5 \\
\hline FBI & 200 & 10 & .35 & 5 & 5 \\
\hline FB2 & 200 & 15 & .35 & 5 & 5 \\
\hline FB3 & 200 & 20 & .35 & 5 & 5 \\
\hline FC0 & 200 & 6.667 & .35 & 6.667 & 6.6667 \\
\hline
\end{tabular}


Table F.1 (Continued)

\begin{tabular}{cccccc}
\hline $\begin{array}{c}\text { Maretial } \\
\text { ID }\end{array}$ & $E_{11}$ & $E_{22}, E_{33}$ & $\begin{array}{c}v_{12}, v_{13}, \\
v_{23}\end{array}$ & $G_{12}, G_{13}$ & $G_{23}$ \\
\hline FCl & 200 & 13.333 & .35 & 6.667 & 6.6667 \\
FC2 & 200 & 20 & .35 & 6.667 & 6.6667 \\
FC3 & 200 & 26.667 & .35 & 6.667 & 6.6667 \\
FD0 & 200 & 10 & .35 & 10 & 10 \\
FD1 & 200 & 20 & .35 & 10 & 10 \\
FD2 & 200 & 30 & .35 & 10 & 10 \\
FD3 & 200 & 40 & .35 & 10 & 10 \\
FE0 & 200 & 20 & .35 & 20 & 20 \\
FE1 & 200 & 40 & .35 & 20 & 20 \\
FE2 & 200 & 60 & .35 & 20 & 20 \\
FE3 & 200 & 80 & .35 & 20 & 20 \\
\hline
\end{tabular}




\section{Appendix G}

\section{VARIATION OF $\alpha$ AND THE ASSOCIATED ERRORS FOR THE MATERIALS OF APPENDIX F}

Table G.1 Results of calculations for short specimens with $b / h=1$.

\begin{tabular}{|c|c|c|c|c|c|}
\hline \multirow[t]{2}{*}{$\begin{array}{l}\text { Material } \\
\text { ID }\end{array}$} & \multirow[t]{2}{*}{$\begin{array}{c}\text { Best } \\
\alpha\end{array}$} & \multicolumn{2}{|c|}{$\begin{array}{l}\% \text { Error based on } \\
\text { the best } \alpha\end{array}$} & \multicolumn{2}{|c|}{$\begin{array}{c}\text { \%Error based on } \\
\alpha=0.7\end{array}$} \\
\hline & & $E$ & $G$ & $E$ & $G$ \\
\hline AA0 & 0.864 & -0.19 & -0.04 & -1.59 & 7.54 \\
\hline AAl & 0.677 & -0.01 & 0.01 & 0.19 & -1.06 \\
\hline AA2 & 0.592 & 0.05 & 0.05 & 0.97 & -4.83 \\
\hline $\mathrm{AA} 3$ & 0.545 & 0.12 & -0.09 & 1.44 & -7.07 \\
\hline $\mathrm{AB} 0$ & 0.841 & -0.21 & 0.08 & -1.17 & 6.76 \\
\hline $\mathrm{AB} 1$ & 0.66 & -0.02 & 0 & 0.26 & -1.86 \\
\hline $\mathrm{AB} 2$ & 0.578 & 0.05 & 0.02 & 0.88 & -5.64 \\
\hline$A B 3$ & 0.531 & 0.1 & -0.07 & 1.25 & -7.87 \\
\hline $\mathrm{ACO}$ & 0.817 & -0.18 & -0.04 & -0.78 & 5.81 \\
\hline $\mathrm{ACl}$ & 0.639 & -0.03 & 0.1 & 0.29 & -2.88 \\
\hline $\mathrm{AC} 2$ & 0.564 & 0.06 & -0.08 & 0.76 & -6.66 \\
\hline AC3 & 0.517 & 0.09 & -0.09 & 1.04 & -8.9 \\
\hline $\mathrm{AD} 0$ & 0.784 & -0.15 & -0.06 & -0.44 & 4.52 \\
\hline $\mathrm{AD}$ & 0.62 & 0 & -0.06 & 0.28 & -4.28 \\
\hline AD2 & 0.545 & 0.05 & -0.01 & 0.59 & -8.1 \\
\hline $\mathrm{AD} 3$ & 0.501 & 0.09 & 0 & 0.78 & -10.34 \\
\hline AE0 & 0.735 & -0.11 & 0.02 & -0.18 & 2.4 \\
\hline $\mathrm{AEl}$ & 0.597 & 0.01 & -0.05 & 0.2 & -6.77 \\
\hline AE2 & 0.534 & 0.06 & -0.01 & 0.36 & -10.67 \\
\hline AE3 & 0.496 & 0.09 & -0.02 & 0.46 & -12.95 \\
\hline BA0 & 0.873 & -0.15 & -0.06 & -1.63 & 7.96 \\
\hline $\mathrm{BAl}$ & 0.686 & 0.05 & -0.07 & 0.17 & -0.71 \\
\hline BA2 & 0.602 & 0.12 & -0.05 & 0.96 & -4.51 \\
\hline BA3 & 0.55 & 0.14 & 0.01 & 1.42 & -6.76 \\
\hline $\mathrm{BBO}$ & 0.852 & -0.16 & 0.01 & -1.2 & 7.25 \\
\hline BBI & 0.667 & 0.01 & 0.09 & 0.24 & -1.44 \\
\hline BB2 & 0.587 & 0.1 & -0.02 & 0.87 & -5.24 \\
\hline BB3 & 0.536 & 0.12 & 0.09 & 1.24 & -7.49 \\
\hline $\mathrm{BC} 0$ & 0.829 & -0.14 & -0.02 & -0.81 & 6.42 \\
\hline $\mathrm{BCl}$ & 0.653 & 0.03 & -0.04 & 0.27 & -2.34 \\
\hline $\mathrm{BC} 2$ & 0.573 & 0.09 & -0.02 & 0.75 & -6.15 \\
\hline
\end{tabular}


Table G. 1 (Continued)

\begin{tabular}{|c|c|c|c|c|c|}
\hline \multirow[t]{2}{*}{$\begin{array}{c}\text { Material } \\
\text { ID }\end{array}$} & \multirow[t]{2}{*}{$\begin{array}{c}\text { Best } \\
\alpha\end{array}$} & \multicolumn{2}{|c|}{$\begin{array}{c}\% \text { Error based on } \\
\text { the best } \alpha\end{array}$} & \multicolumn{2}{|c|}{$\begin{array}{c}\text { \%Error based on } \\
\alpha=0.7\end{array}$} \\
\hline & & $E$ & $G$ & $E$ & $G$ \\
\hline BC3 & 0.527 & 0.13 & -0.04 & 1.03 & -8.4 \\
\hline BD0 & 0.798 & -0.12 & 0.02 & -0.47 & 5.38 \\
\hline BD1 & 0.634 & 0.04 & -0.02 & 0.27 & -3.5 \\
\hline BD2 & 0.559 & 0.09 & 0.03 & 0.58 & -7.34 \\
\hline BD3 & 0.517 & 0.13 & -0.08 & 0.77 & -9.6 \\
\hline BE0 & 0.759 & -0.08 & 0 & -0.19 & 3.98 \\
\hline BEl & 0.62 & 0.04 & -0.03 & 0.19 & -5.26 \\
\hline BE2 & 0.557 & 0.09 & 0.03 & 0.35 & -9.18 \\
\hline BE3 & 0.522 & 0.12 & -0.09 & 0.45 & -11.47 \\
\hline CAO & 0.883 & -0.1 & -0.08 & -1.66 & 8.37 \\
\hline CAl & 0.691 & 0.07 & 0.06 & 0.15 & -0.37 \\
\hline $\mathrm{CA} 2$ & 0.606 & 0.14 & 0.05 & 0.94 & -4.19 \\
\hline $\mathrm{CA} 3$ & 0.555 & 0.17 & 0.09 & 1.41 & -6.46 \\
\hline CBO & 0.864 & -0.11 & -0.08 & -1.23 & 7.74 \\
\hline CB 1 & 0.677 & 0.06 & 0.07 & 0.22 & -1.03 \\
\hline CB2 & 0.597 & 0.15 & -0.06 & 0.85 & -4.85 \\
\hline CB3 & 0.545 & 0.17 & 0.04 & 1.23 & -7.12 \\
\hline $\mathrm{CCO}$ & 0.841 & -0.11 & 0.01 & -0.83 & 7.03 \\
\hline $\mathrm{CCl}$ & 0.663 & 0.06 & 0.04 & 0.26 & -1.8 \\
\hline CC2 & 0.583 & 0.13 & 0.04 & 0.74 & -5.64 \\
\hline CC3 & 0.536 & 0.17 & 0.01 & 1.02 & -7.9 \\
\hline CD0 & 0.815 & -0.08 & -0.03 & -0.48 & 6.23 \\
\hline CD1 & 0.648 & 0.07 & 0.02 & 0.25 & -2.72 \\
\hline CD2 & 0.573 & 0.13 & 0.06 & 0.57 & -6.58 \\
\hline CD3 & 0.531 & 0.17 & -0.05 & 0.76 & -8.85 \\
\hline CEO & 0.782 & -0.05 & -0.02 & -0.2 & 5.58 \\
\hline CEl & 0.644 & 0.08 & -0.02 & 0.18 & -3.73 \\
\hline CE2 & 0.583 & 0.13 & -0.07 & 0.34 & -7.68 \\
\hline CE3 & 0.545 & 0.16 & -0.04 & 0.44 & -9.98 \\
\hline DA0 & 0.864 & -0.22 & 0.08 & -1.62 & 7.65 \\
\hline DAl & 0.681 & 0.01 & -0.09 & 0.17 & -0.95 \\
\hline DA2 & 0.597 & 0.07 & -0.05 & 0.95 & -4.72 \\
\hline DA3 & 0.545 & 0.1 & 0.01 & 1.42 & -6.96 \\
\hline DB0 & 0.845 & -0.2 & -0.03 & -1.19 & 6.89 \\
\hline DB 1 & 0.663 & -0.02 & 0.01 & 0.24 & -1.74 \\
\hline DB2 & 0.583 & 0.06 & -0.07 & 0.86 & -5.52 \\
\hline DB3 & 0.531 & 0.08 & 0.04 & 1.24 & -7.76 \\
\hline DC0 & 0.82 & -0.18 & -0.02 & -0.8 & 5.94 \\
\hline
\end{tabular}


Table G. 1 (Continued)

\begin{tabular}{|c|c|c|c|c|c|}
\hline \multirow[t]{2}{*}{$\begin{array}{l}\text { Material } \\
\text { ID }\end{array}$} & \multirow[t]{2}{*}{$\begin{array}{c}\text { Best } \\
\alpha\end{array}$} & \multicolumn{2}{|c|}{$\begin{array}{l}\% \text { Error based on } \\
\text { the best } \alpha\end{array}$} & \multicolumn{2}{|c|}{$\begin{array}{c}\text { \%Error based on } \\
\alpha=0.7\end{array}$} \\
\hline & & $E$ & $G$ & $E$ & $G$ \\
\hline $\mathrm{DCl}$ & 0.644 & -0.02 & 0 & 0.28 & -2.75 \\
\hline DC2 & 0.564 & 0.04 & 0.04 & 0.75 & -6.53 \\
\hline DC3 & 0.517 & 0.08 & 0.02 & 1.03 & -8.77 \\
\hline DD0 & 0.784 & -0.16 & 0.1 & -0.46 & 4.68 \\
\hline DD1 & 0.62 & -0.01 & 0.08 & 0.27 & -4.13 \\
\hline DD2 & 0.55 & 0.06 & -0.09 & 0.58 & -7.95 \\
\hline DD3 & 0.503 & 0.08 & 0.02 & 0.77 & -10.19 \\
\hline DE0 & 0.737 & -0.12 & 0.07 & -0.18 & 2.6 \\
\hline $\mathrm{DE} 1$ & 0.599 & 0.01 & 0.01 & 0.19 & -6.56 \\
\hline DE2 & 0.536 & 0.06 & 0.05 & 0.36 & -10.46 \\
\hline DE3 & 0.498 & 0.09 & 0.05 & 0.45 & -12.73 \\
\hline EA0 & 0.873 & -0.18 & 0.1 & -1.66 & 8.1 \\
\hline EAl & 0.686 & 0.02 & 0.07 & 0.14 & -0.57 \\
\hline EA2 & 0.602 & 0.09 & 0.08 & 0.93 & -4.37 \\
\hline EA3 & 0.555 & 0.16 & -0.07 & 1.4 & -6.63 \\
\hline EB0 & 0.855 & -0.17 & 0.05 & -1.23 & 7.41 \\
\hline EB1 & 0.672 & 0.02 & 0.03 & 0.21 & -1.29 \\
\hline EB2 & 0.592 & 0.11 & -0.08 & 0.85 & -5.1 \\
\hline EB3 & 0.541 & 0.13 & 0.02 & 1.22 & -7.35 \\
\hline $\mathrm{EC} 0$ & 0.831 & -0.15 & 0.04 & -0.83 & 6.58 \\
\hline $\mathrm{ECl}$ & 0.655 & 0.03 & 0 & 0.26 & -2.17 \\
\hline EC2 & 0.578 & 0.1 & -0.08 & 0.73 & -5.99 \\
\hline EC3 & 0.529 & 0.13 & 0 & 1.01 & -8.24 \\
\hline ED0 & 0.803 & -0.12 & -0.05 & -0.48 & 5.57 \\
\hline ED1 & 0.639 & 0.04 & -0.08 & 0.25 & -3.31 \\
\hline ED2 & 0.564 & 0.09 & -0.02 & 0.57 & -7.15 \\
\hline ED3 & 0.517 & 0.12 & 0.09 & 0.76 & -9.41 \\
\hline EE0 & 0.761 & -0.09 & 0.1 & -0.2 & 4.24 \\
\hline EE1 & 0.625 & 0.05 & -0.07 & 0.18 & -4.99 \\
\hline EE2 & 0.562 & 0.09 & 0.01 & 0.34 & -8.91 \\
\hline EE3 & 0.524 & 0.12 & 0.03 & 0.44 & -11.20 \\
\hline FA0 & 0.885 & -0.12 & -0.01 & -1.7 & 8.55 \\
\hline FAl & 0.695 & 0.07 & 0.02 & 0.11 & -0.19 \\
\hline FA2 & 0.611 & 0.15 & 0.01 & 0.91 & -4.03 \\
\hline FA3 & 0.559 & 0.18 & 0.05 & 1.38 & -6.29 \\
\hline FB0 & 0.866 & -0.12 & 0.01 & -1.26 & 7.93 \\
\hline FBI & 0.681 & 0.06 & 0.04 & 0.19 & -0.84 \\
\hline
\end{tabular}


Table G.1 (Continued)

\begin{tabular}{ccccccc}
\hline $\begin{array}{c}\text { Material } \\
\text { ID }\end{array}$ & $\begin{array}{c}\text { Best } \\
\alpha\end{array}$ & \multicolumn{2}{c}{$\begin{array}{c}\text { \%Error based on } \\
\text { the best } \alpha\end{array}$} & \multicolumn{2}{c}{$\begin{array}{c}\text { \%Error based on } \\
\alpha=0.7\end{array}$} \\
\cline { 3 - 4 } \cline { 6 - 6 } \cline { 5 - 6 } & & $E$ & $G$ & & $E$ & $G$ \\
\hline FB2 & 0.602 & 0.15 & -0.09 & & 0.83 & -4.68 \\
FB3 & 0.55 & 0.18 & 0 & & 1.2 & -6.94 \\
FC0 & 0.845 & -0.11 & -0.03 & & -0.86 & 7.23 \\
FC1 & 0.667 & 0.06 & 0.01 & & 0.23 & -1.6 \\
FC2 & 0.587 & 0.13 & 0.02 & & 0.71 & -5.44 \\
FC3 & 0.541 & 0.17 & -0.02 & & 1 & -7.71 \\
FD0 & 0.817 & -0.09 & 0.08 & & -0.5 & 6.47 \\
FD1 & 0.653 & 0.07 & 0.01 & & 0.23 & -2.49 \\
FD2 & 0.578 & 0.13 & 0.05 & & 0.56 & -6.35 \\
FD3 & 0.536 & 0.17 & -0.06 & & 0.74 & -8.63 \\
FE0 & 0.787 & -0.06 & -0.04 & & -0.21 & 5.89 \\
FE1 & 0.648 & 0.07 & 0 & & 0.17 & -3.41 \\
FE2 & 0.587 & 0.13 & -0.04 & & 0.33 & -7.35 \\
FE3 & 0.55 & 0.15 & 0.01 & 0.43 & -9.64 \\
\hline
\end{tabular}


Table G.2 Results of calculations for long specimens with $b / h=1$.

\begin{tabular}{|c|c|c|c|c|c|}
\hline \multirow[t]{2}{*}{$\begin{array}{l}\text { Material } \\
\text { ID }\end{array}$} & \multirow[t]{2}{*}{$\begin{array}{c}\text { Best } \\
\alpha\end{array}$} & \multicolumn{2}{|c|}{$\begin{array}{l}\% \text { Error based on } \\
\text { the best } \alpha\end{array}$} & \multicolumn{2}{|c|}{$\begin{array}{c}\text { \%Error based on } \\
\alpha=0.7\end{array}$} \\
\hline & & $E$ & $G$ & $E$ & $G$ \\
\hline AA0 & 0.855 & -0.11 & 0 & -0.54 & 4.99 \\
\hline AAl & 0.677 & -0.03 & 0.03 & 0.04 & -0.71 \\
\hline AA2 & 0.597 & 0.01 & 0.03 & 0.3 & -3.2 \\
\hline AA3 & 0.55 & 0.03 & 0 & 0.45 & -4.68 \\
\hline ABO & 0.831 & -0.11 & 0.02 & -0.4 & 4.42 \\
\hline $\mathrm{AB} 1$ & 0.663 & -0.01 & -0.09 & 0.08 & -1.32 \\
\hline AB2 & 0.583 & 0.02 & 0 & 0.28 & -3.81 \\
\hline AB3 & 0.536 & 0.03 & 0.01 & 0.4 & -5.29 \\
\hline $\mathrm{ACO}$ & 0.803 & -0.09 & 0.03 & -0.27 & 3.71 \\
\hline $\mathrm{ACl}$ & 0.639 & -0.01 & 0.05 & 0.09 & -2.07 \\
\hline AC2 & 0.569 & 0.02 & -0.07 & 0.25 & -4.59 \\
\hline AC3 & 0.522 & 0.03 & 0.02 & 0.33 & -6.06 \\
\hline $\mathrm{AD0}$ & 0.77 & -0.07 & -0.08 & -0.15 & 2.73 \\
\hline ADl & 0.62 & 0 & -0.05 & 0.09 & -3.14 \\
\hline AD2 & 0.55 & 0.02 & 0.04 & 0.19 & -5.67 \\
\hline AD3 & 0.513 & 0.04 & -0.07 & 0.25 & -7.16 \\
\hline $\mathrm{AE} 0$ & 0.719 & -0.05 & 0.02 & -0.06 & 1 \\
\hline $\mathrm{AE} 1$ & 0.597 & 0 & 0.06 & 0.06 & -5.13 \\
\hline $\mathrm{AE} 2$ & 0.545 & 0.03 & -0.07 & 0.12 & -7.73 \\
\hline $\mathrm{AE} 3$ & 0.513 & 0.04 & -0.06 & 0.15 & -9.24 \\
\hline BAO & 0.864 & -0.1 & 0.06 & -0.55 & 5.35 \\
\hline BAI & 0.686 & -0.01 & 0.05 & 0.03 & -0.4 \\
\hline $\mathrm{BA} 2$ & 0.606 & 0.03 & 0.03 & 0.29 & -2.91 \\
\hline BA3 & 0.559 & 0.05 & 0 & 0.44 & -4.4 \\
\hline BBO & 0.845 & -0.08 & -0.04 & -0.4 & 4.84 \\
\hline BB1 & 0.672 & 0 & -0.01 & 0.07 & -0.93 \\
\hline BB2 & 0.597 & 0.04 & -0.09 & 0.27 & -3.45 \\
\hline BB3 & 0.55 & 0.06 & -0.08 & 0.39 & -4.93 \\
\hline $\mathrm{BCO}$ & 0.817 & -0.08 & 0.06 & -0.27 & 4.25 \\
\hline $\mathrm{BCl}$ & 0.653 & 0.01 & 0.05 & 0.09 & -1.58 \\
\hline $\mathrm{BC} 2$ & 0.578 & 0.04 & 0.09 & 0.24 & -4.11 \\
\hline $\mathrm{BC} 3$ & 0.536 & 0.06 & 0.01 & 0.33 & -5.6 \\
\hline BD0 & 0.789 & -0.06 & -0.06 & -0.16 & 3.52 \\
\hline BDI & 0.639 & 0.01 & -0.04 & 0.08 & -2.41 \\
\hline BD2 & 0.569 & 0.04 & 0.05 & 0.19 & -4.96 \\
\hline BD3 & 0.531 & 0.06 & -0.06 & 0.25 & -6.46 \\
\hline BE0 & 0.747 & -0.03 & -0.04 & -0.06 & 2.44 \\
\hline
\end{tabular}


Table G.2 (Continued)

\begin{tabular}{|c|c|c|c|c|c|}
\hline \multirow[t]{2}{*}{$\begin{array}{l}\text { Material } \\
\text { ID }\end{array}$} & \multirow[t]{2}{*}{$\begin{array}{c}\text { Best } \\
\alpha\end{array}$} & \multicolumn{2}{|c|}{$\begin{array}{l}\% \text { Error based on } \\
\text { the best } \alpha\end{array}$} & \multicolumn{2}{|c|}{$\begin{array}{c}\text { \%Error based on } \\
\alpha=0.7\end{array}$} \\
\hline & & $E$ & $G$ & $E$ & $G$ \\
\hline $\mathrm{BE} 1$ & 0.625 & 0.02 & 0.09 & 0.06 & -3.72 \\
\hline BE2 & 0.573 & 0.04 & 0 & 0.12 & -6.32 \\
\hline BE3 & 0.541 & 0.05 & 0.03 & 0.15 & -7.84 \\
\hline CA0 & 0.878 & -0.07 & -0.05 & -0.56 & 5.7 \\
\hline $\mathrm{CAl}$ & 0.7 & 0.03 & -0.09 & 0.03 & -0.09 \\
\hline $\mathrm{CA} 2$ & 0.616 & 0.05 & 0.03 & 0.29 & -2.62 \\
\hline $\mathrm{CA} 3$ & 0.569 & 0.08 & -0.01 & 0.44 & -4.11 \\
\hline CBO & 0.859 & -0.06 & -0.09 & -0.42 & 5.27 \\
\hline $\mathrm{CB} 1$ & 0.681 & 0.02 & 0.06 & 0.06 & -0.56 \\
\hline CB2 & 0.606 & 0.06 & -0.04 & 0.27 & -3.09 \\
\hline CB3 & 0.559 & 0.07 & -0.02 & 0.39 & -4.58 \\
\hline $\mathrm{CCO}$ & 0.831 & -0.06 & 0.1 & -0.28 & 4.8 \\
\hline $\mathrm{CCl}$ & 0.672 & 0.03 & -0.1 & 0.08 & -1.08 \\
\hline $\mathrm{CC} 2$ & 0.597 & 0.06 & -0.07 & 0.24 & -3.63 \\
\hline $\mathrm{CC} 3$ & 0.55 & 0.07 & 0.02 & 0.33 & -5.12 \\
\hline CD0 & 0.808 & -0.04 & -0.05 & -0.16 & 4.29 \\
\hline $\mathrm{CD} 1$ & 0.658 & 0.03 & -0.04 & 0.08 & -1.69 \\
\hline $\mathrm{CD} 2$ & 0.587 & 0.06 & 0.07 & 0.19 & -4.25 \\
\hline $\mathrm{CD} 3$ & 0.55 & 0.08 & -0.04 & 0.25 & -5.76 \\
\hline CE0 & 0.775 & -0.02 & -0.07 & -0.07 & 3.93 \\
\hline $\mathrm{CEl}$ & 0.655 & 0.03 & 0.01 & 0.06 & -2.28 \\
\hline CE2 & 0.602 & 0.06 & 0.06 & 0.11 & -4.91 \\
\hline CE3 & 0.571 & 0.07 & 0 & 0.15 & -6.44 \\
\hline DA0 & 0.859 & -0.1 & -0.08 & -0.55 & 5.06 \\
\hline DAl & 0.681 & -0.02 & -0.04 & 0.03 & -0.63 \\
\hline DA2 & 0.602 & 0.02 & -0.04 & 0.29 & -3.13 \\
\hline DA3 & 0.55 & 0.03 & 0.07 & 0.44 & -4.61 \\
\hline DB0 & 0.831 & -0.11 & 0.1 & -0.4 & 4.5 \\
\hline DB 1 & 0.663 & -0.02 & 0 & 0.06 & -1.23 \\
\hline DB2 & 0.587 & 0.02 & -0.08 & 0.27 & -3.74 \\
\hline DB3 & 0.541 & 0.04 & -0.06 & 0.39 & -5.21 \\
\hline DC0 & 0.808 & -0.09 & -0.06 & -0.27 & 3.79 \\
\hline $\mathrm{DCl}$ & 0.644 & -0.01 & -0.02 & 0.08 & -1.98 \\
\hline DC2 & 0.569 & 0.02 & 0.02 & 0.24 & -4.49 \\
\hline DC3 & 0.522 & 0.03 & 0.09 & 0.33 & -5.98 \\
\hline DD0 & 0.77 & -0.08 & 0.03 & -0.16 & 2.84 \\
\hline DDI & 0.62 & 0 & 0.05 & 0.09 & -3.04 \\
\hline DD2 & 0.555 & 0.03 & -0.02 & 0.19 & -5.57 \\
\hline
\end{tabular}


Table G.2 (Continued)

\begin{tabular}{|c|c|c|c|c|c|}
\hline \multirow[t]{2}{*}{$\begin{array}{l}\text { Material } \\
\text { ID }\end{array}$} & \multirow[t]{2}{*}{$\begin{array}{c}\text { Best } \\
\alpha\end{array}$} & \multicolumn{2}{|c|}{$\begin{array}{c}\% \text { Error based on } \\
\text { the best } \alpha\end{array}$} & \multicolumn{2}{|c|}{$\begin{array}{c}\% \text { Error based on } \\
\alpha=0.7\end{array}$} \\
\hline & & $E$ & $G$ & $E$ & $\vec{G}$ \\
\hline DD3 & 0.513 & 0.04 & 0.03 & 0.25 & -7.05 \\
\hline DE0 & 0.721 & -0.05 & 0.01 & -0.06 & 1.12 \\
\hline DEI & 0.602 & 0 & -0.02 & 0.06 & -4.99 \\
\hline DE2 & 0.545 & 0.03 & 0.07 & 0.12 & -7.58 \\
\hline DE3 & 0.513 & 0.04 & 0.09 & 0.15 & -9.08 \\
\hline EA0 & 0.869 & -0.1 & 0 & -0.57 & 5.45 \\
\hline EAl & 0.691 & 0 & 0 & 0.02 & -0.3 \\
\hline $\mathrm{EA} 2$ & 0.611 & 0.03 & -0.02 & 0.28 & -2.81 \\
\hline EA3 & 0.559 & 0.04 & 0.09 & 0.43 & -4.3 \\
\hline EB0 & 0.85 & -0.08 & -0.1 & -0.42 & 4.95 \\
\hline EB1 & 0.672 & 0 & 0.09 & 0.06 & -0.83 \\
\hline EB2 & 0.597 & 0.04 & 0.01 & 0.27 & -3.35 \\
\hline EB3 & 0.55 & 0.06 & 0.01 & 0.39 & -4.84 \\
\hline EC0 & 0.822 & -0.07 & 0 & -0.28 & 4.36 \\
\hline $\mathrm{ECl}$ & 0.658 & 0.01 & 0.01 & 0.08 & -1.47 \\
\hline $\mathrm{EC} 2$ & 0.583 & 0.04 & 0.04 & 0.24 & -4 \\
\hline EC3 & 0.541 & 0.06 & -0.03 & 0.33 & -5.49 \\
\hline ED0 & 0.789 & -0.06 & 0.07 & -0.16 & 3.64 \\
\hline EDI & 0.644 & 0.01 & -0.08 & 0.08 & -2.27 \\
\hline ED2 & 0.573 & 0.04 & 0.01 & 0.19 & -4.83 \\
\hline ED3 & 0.531 & 0.05 & 0.07 & 0.25 & -6.32 \\
\hline EE0 & 0.752 & -0.04 & -0.09 & -0.07 & 2.64 \\
\hline EEl & 0.63 & 0.02 & 0.06 & 0.06 & -3.52 \\
\hline EE2 & 0.578 & 0.04 & -0.02 & 0.11 & -6.12 \\
\hline EE3 & 0.545 & 0.05 & 0.02 & 0.14 & -7.64 \\
\hline FA0 & 0.878 & -0.08 & 0.07 & -0.57 & 5.82 \\
\hline FAl & 0.7 & 0.02 & 0.03 & 0.02 & 0.03 \\
\hline FA2 & 0.62 & 0.06 & 0 & 0.28 & -2.5 \\
\hline FA3 & 0.573 & 0.08 & -0.04 & 0.43 & -4 \\
\hline FB0 & 0.859 & -0.07 & 0.04 & -0.43 & 5.4 \\
\hline FBI & 0.686 & 0.02 & 0.03 & 0.05 & -0.43 \\
\hline FB2 & 0.606 & 0.05 & 0.09 & 0.26 & -2.96 \\
\hline FB3 & 0.559 & 0.07 & 0.09 & 0.38 & -4.46 \\
\hline $\mathrm{FCO}$ & 0.836 & -0.06 & 0.06 & -0.29 & 4.93 \\
\hline $\mathrm{FCl}$ & 0.672 & 0.02 & 0.04 & 0.07 & -0.94 \\
\hline $\mathrm{FC} 2$ & 0.597 & 0.06 & 0.07 & 0.23 & -3.49 \\
\hline $\mathrm{FC} 3$ & 0.555 & 0.08 & -0.01 & 0.32 & -4.99 \\
\hline FD0 & 0.813 & -0.05 & -0.07 & -0.17 & 4.46 \\
\hline
\end{tabular}


Table G.2 (Continued)

\begin{tabular}{ccccccc}
\hline $\begin{array}{c}\text { Material } \\
\text { ID }\end{array}$ & $\begin{array}{c}\text { Best } \\
\alpha\end{array}$ & \multicolumn{2}{c}{$\begin{array}{c}\text { \%Error based on } \\
\text { the best } \alpha\end{array}$} & & \multicolumn{2}{c}{$\begin{array}{c}\text { \%Error based on } \\
\alpha=0.7\end{array}$} \\
\cline { 3 - 4 } \cline { 5 - 6 } & & $E$ & $G$ & & $E$ & $G$ \\
\hline FD1 & 0.663 & 0.04 & -0.07 & & 0.08 & -1.53 \\
FD2 & 0.592 & 0.06 & 0.05 & & 0.18 & -4.09 \\
FD3 & 0.555 & 0.08 & -0.06 & & 0.24 & -5.6 \\
FE0 & 0.78 & -0.03 & -0.09 & & -0.07 & 4.17 \\
FE1 & 0.66 & 0.03 & 0 & & 0.06 & -2.05 \\
FE2 & 0.606 & 0.06 & 0.07 & & 0.11 & -4.67 \\
FE3 & 0.578 & 0.07 & -0.08 & & 0.14 & -6.18 \\
\hline
\end{tabular}


Table G.3 Results of calculations for short specimens with $b / h=3$.

\begin{tabular}{ccccccc}
\hline Material & Best & \multicolumn{2}{c}{$\begin{array}{c}\text { \%Error based on } \\
\text { the best } \alpha\end{array}$} & \multicolumn{2}{c}{$\begin{array}{c}\text { \%Error based on } \\
\alpha=0.7\end{array}$} \\
\cline { 3 - 4 } \cline { 6 - 7 } & $\alpha$ & $E$ & $G$ & & $E$ & $G$ \\
\hline AA0 & 0.869 & 0.04 & -0.01 & & -1.4 & 7.78 \\
AA1 & 0.681 & 0.2 & 0.1 & & 0.36 & -0.76 \\
AA2 & 0.602 & 0.29 & -0.06 & & 1.12 & -4.52 \\
AA3 & 0.55 & 0.3 & 0 & & 1.58 & -6.76 \\
AB0 & 0.85 & 0.04 & -0.07 & & -0.98 & 7.07 \\
AB1 & 0.667 & 0.19 & 0.07 & & 0.42 & -1.46 \\
AB2 & 0.587 & 0.26 & 0.01 & & 1.03 & -5.22 \\
AB3 & 0.541 & 0.31 & -0.07 & & 1.4 & -7.45 \\
AC0 & 0.827 & 0.05 & -0.1 & & -0.6 & 6.22 \\
AC1 & 0.653 & 0.21 & -0.02 & & 0.45 & -2.31 \\
AC2 & 0.573 & 0.26 & 0.08 & & 0.91 & -6.05 \\
AC3 & 0.527 & 0.29 & 0.08 & & 1.18 & -8.27 \\
AD0 & 0.794 & 0.06 & 0.08 & & -0.26 & 5.17 \\
AD1 & 0.637 & 0.23 & 0.01 & & 0.45 & -3.35 \\
AD2 & 0.564 & 0.28 & 0.07 & & 0.76 & -7.06 \\
AD3 & 0.522 & 0.32 & 0.02 & & 0.94 & -9.25 \\
AE0 & 0.759 & 0.13 & -0.05 & & 0.03 & 3.92 \\
AE1 & 0.632 & 0.28 & -0.02 & & 0.41 & -4.49 \\
AE2 & 0.576 & 0.34 & -0.01 & & 0.57 & -8.05 \\
AE3 & 0.541 & 0.38 & 0.07 & & 0.66 & -10.14 \\
BA0 & 0.878 & 0.13 & 0 & & -1.39 & 8.23 \\
BA1 & 0.691 & 0.3 & 0.04 & & 0.38 & -0.39 \\
BA2 & 0.606 & 0.36 & 0.07 & & 1.16 & -4.17 \\
BA3 & 0.559 & 0.41 & -0.07 & & 1.61 & -6.42 \\
BB0 & 0.859 & 0.12 & 0.03 & & -0.97 & 7.61 \\
BB1 & 0.679 & 0.3 & -0.01 & & 0.45 & -0.99 \\
BB2 & 0.597 & 0.36 & 0.03 & & 1.06 & -4.76 \\
BB3 & 0.55 & 0.4 & -0.06 & & 1.43 & -7 \\
BC0 & 0.838 & 0.13 & 0.02 & & -0.59 & 6.92 \\
BC1 & 0.667 & 0.31 & -0.06 & & 0.48 & -1.67 \\
BC2 & 0.587 & 0.36 & 0.04 & & 0.94 & -5.42 \\
BC3 & 0.541 & 0.39 & 0.04 & & 1.22 & -7.64 \\
BD0 & 0.813 & 0.15 & 0.07 & & -0.24 & 6.2 \\
BD1 & 0.658 & 0.33 & -0.1 & & 0.48 & -2.34 \\
BD2 & 0.585 & 0.39 & -0.01 & & 0.79 & -6.04 \\
BD3 & 0.541 & 0.42 & 0.08 & & 0.98 & -8.23 \\
BE0 & 0.789 & 0.23 & -0.07 & & 0.07 & 6.02 \\
\hline & & & & & \\
\hline
\end{tabular}


Table G.3 (Continued)

\begin{tabular}{|c|c|c|c|c|c|}
\hline \multirow[t]{2}{*}{$\begin{array}{l}\text { Material } \\
\text { ID }\end{array}$} & \multirow[t]{2}{*}{$\begin{array}{c}\text { Best } \\
\alpha\end{array}$} & \multicolumn{2}{|c|}{$\begin{array}{l}\text { \%Error based on } \\
\text { the best } \alpha\end{array}$} & \multicolumn{2}{|c|}{$\begin{array}{c}\text { \%Error based on } \\
\alpha=0.7\end{array}$} \\
\hline & & $E$ & $G$ & $E$ & $G$ \\
\hline BEI & 0.667 & 0.4 & -0.09 & 0.46 & -2.27 \\
\hline BE2 & 0.611 & 0.47 & 0.07 & 0.63 & -5.74 \\
\hline BE3 & 0.58 & 0.51 & -0.04 & 0.73 & -7.78 \\
\hline CAO & 0.888 & 0.21 & 0 & -1.39 & 8.67 \\
\hline $\mathrm{CAl}$ & 0.7 & 0.41 & -0.04 & 0.41 & -0.04 \\
\hline CA2 & 0.616 & 0.47 & -0.02 & 1.19 & -3.85 \\
\hline CA3 & 0.564 & 0.49 & 0.03 & 1.65 & -6.1 \\
\hline CB0 & 0.871 & 0.21 & 0 & -0.96 & 8.15 \\
\hline CBI & 0.691 & 0.41 & -0.1 & 0.48 & -0.54 \\
\hline CB2 & 0.606 & 0.46 & 0.03 & 1.1 & -4.33 \\
\hline CB3 & 0.559 & 0.5 & -0.06 & 1.47 & -6.57 \\
\hline $\mathrm{CCO}$ & 0.852 & 0.22 & 0.01 & -0.57 & 7.62 \\
\hline $\mathrm{CCl}$ & 0.679 & 0.41 & 0 & 0.52 & -1.03 \\
\hline $\mathrm{CC} 2$ & 0.602 & 0.47 & -0.01 & 0.98 & -4.79 \\
\hline $\mathrm{CC} 3$ & 0.555 & 0.51 & 0 & 1.26 & -7.01 \\
\hline CD0 & 0.831 & 0.24 & 0.08 & -0.21 & 7.25 \\
\hline CDI & 0.677 & 0.44 & -0.07 & 0.52 & -1.32 \\
\hline CD2 & 0.606 & 0.51 & -0.07 & 0.84 & -5.01 \\
\hline CD3 & 0.564 & 0.55 & -0.07 & 1.02 & -7.19 \\
\hline CE0 & 0.82 & 0.33 & -0.03 & 0.12 & 8.19 \\
\hline CEl & 0.7 & 0.52 & 0.05 & 0.52 & 0.05 \\
\hline CE2 & 0.648 & 0.6 & 0.07 & 0.7 & -3.33 \\
\hline CE3 & 0.62 & 0.66 & -0.08 & 0.8 & -5.29 \\
\hline DA0 & 0.873 & 0.04 & 0.07 & -1.44 & 8.08 \\
\hline DAl & 0.691 & 0.23 & 0 & 0.31 & -0.43 \\
\hline DA2 & 0.606 & 0.29 & 0.06 & 1.08 & -4.19 \\
\hline DA3 & 0.559 & 0.34 & -0.07 & 1.54 & -6.42 \\
\hline DBO & 0.855 & 0.04 & 0.02 & -1.02 & 7.38 \\
\hline DBI & 0.677 & 0.22 & -0.01 & 0.38 & -1.11 \\
\hline DB2 & 0.597 & 0.29 & -0.05 & 1 & -4.85 \\
\hline DB3 & 0.545 & 0.31 & 0.08 & 1.37 & -7.07 \\
\hline DC0 & 0.831 & 0.05 & 0.02 & -0.63 & 6.56 \\
\hline $\mathrm{DCl}$ & 0.663 & 0.23 & -0.07 & 0.43 & -1.91 \\
\hline DC2 & 0.583 & 0.28 & 0.06 & 0.89 & -5.62 \\
\hline DC3 & 0.536 & 0.32 & 0.08 & 1.16 & -7.82 \\
\hline DD0 & 0.803 & 0.08 & -0.04 & -0.28 & 5.57 \\
\hline DD1 & 0.646 & 0.24 & 0.01 & 0.43 & -2.85 \\
\hline DD2 & 0.578 & 0.32 & -0.09 & 0.74 & -6.5 \\
\hline
\end{tabular}


Table G.3 (Continued)

\begin{tabular}{|c|c|c|c|c|c|}
\hline \multirow[t]{2}{*}{$\begin{array}{l}\text { Material } \\
\text { ID }\end{array}$} & \multirow[t]{2}{*}{$\begin{array}{l}\text { Best } \\
\alpha\end{array}$} & \multicolumn{2}{|c|}{$\begin{array}{c}\% \text { Error based on } \\
\text { the best } \alpha\end{array}$} & \multicolumn{2}{|c|}{$\begin{array}{c}\text { \%Error based on } \\
\alpha=0.7\end{array}$} \\
\hline & & $E$ & $G$ & $E$ & $G$ \\
\hline DD3 & 0.536 & 0.35 & -0.1 & 0.93 & -8.66 \\
\hline DE0 & 0.766 & 0.14 & 0.04 & 0.02 & 4.5 \\
\hline DEl & 0.644 & 0.3 & 0.03 & 0.4 & -3.68 \\
\hline DE2 & 0.592 & 0.37 & -0.08 & 0.57 & -7.09 \\
\hline DE3 & 0.559 & 0.41 & -0.01 & 0.67 & -9.07 \\
\hline EA0 & 0.888 & 0.15 & -0.07 & -1.45 & 8.6 \\
\hline EAl & 0.7 & 0.33 & 0.02 & 0.33 & 0.02 \\
\hline EA2 & 0.616 & 0.39 & 0.06 & 1.1 & -3.76 \\
\hline EA3 & 0.569 & 0.45 & -0.08 & 1.57 & -6.01 \\
\hline EB0 & 0.869 & 0.14 & -0.03 & -1.02 & 8 \\
\hline EB1 & 0.688 & 0.32 & 0 & 0.4 & -0.55 \\
\hline EB2 & 0.606 & 0.38 & 0.06 & 1.02 & -4.3 \\
\hline EB3 & 0.559 & 0.43 & -0.02 & 1.39 & -6.54 \\
\hline $\mathrm{EC} 0$ & 0.845 & 0.13 & 0.1 & -0.62 & 7.35 \\
\hline $\mathrm{ECl}$ & 0.677 & 0.33 & -0.02 & 0.45 & -1.16 \\
\hline EC2 & 0.602 & 0.41 & -0.09 & 0.92 & -4.88 \\
\hline EC3 & 0.555 & 0.44 & -0.06 & 1.19 & -7.09 \\
\hline ED0 & 0.822 & 0.17 & 0.06 & -0.26 & 6.7 \\
\hline ED1 & 0.667 & 0.35 & 0.03 & 0.46 & -1.72 \\
\hline ED2 & 0.597 & 0.42 & 0.08 & 0.78 & -5.35 \\
\hline ED3 & 0.555 & 0.46 & 0.1 & 0.96 & -7.5 \\
\hline EE0 & 0.798 & 0.24 & -0.01 & 0.06 & 6.73 \\
\hline EE1 & 0.681 & 0.42 & -0.01 & 0.46 & -1.26 \\
\hline EE2 & 0.63 & 0.5 & 0.07 & 0.63 & -4.54 \\
\hline EE3 & 0.602 & 0.56 & -0.03 & 0.74 & -6.44 \\
\hline FA0 & 0.897 & 0.23 & 0.01 & -1.45 & 9.12 \\
\hline FAl & 0.709 & 0.43 & 0.03 & 0.35 & 0.45 \\
\hline FA2 & 0.625 & 0.49 & 0.06 & 1.13 & -3.34 \\
\hline FA3 & 0.578 & 0.56 & -0.09 & 1.6 & -5.6 \\
\hline FB0 & 0.883 & 0.24 & -0.1 & -1.02 & 8.62 \\
\hline FBI & 0.7 & 0.43 & 0 & 0.43 & 0 \\
\hline FB2 & 0.62 & 0.51 & -0.06 & 1.05 & -3.77 \\
\hline FB3 & 0.569 & 0.52 & 0.07 & 1.42 & -6.01 \\
\hline $\mathrm{FC} 0$ & 0.864 & 0.24 & -0.07 & -0.61 & 8.14 \\
\hline $\mathrm{FCl}$ & 0.691 & 0.43 & 0.04 & 0.48 & -0.42 \\
\hline FC2 & 0.616 & 0.51 & -0.03 & 0.95 & -4.14 \\
\hline FC3 & 0.569 & 0.55 & 0 & 1.23 & -6.34 \\
\hline FD0 & 0.843 & 0.26 & 0.03 & -0.24 & 7.86 \\
\hline
\end{tabular}


Table G.3 (Continued)

\begin{tabular}{ccccccc}
\hline $\begin{array}{c}\text { Material } \\
\text { ID }\end{array}$ & $\begin{array}{c}\text { Best } \\
\alpha\end{array}$ & \multicolumn{2}{c}{$\begin{array}{c}\text { \%Error based on } \\
\text { the best } \alpha\end{array}$} & & \multicolumn{2}{c}{$\begin{array}{c}\text { \%Error based on } \\
\alpha=0.7\end{array}$} \\
\cline { 3 - 4 } \cline { 6 - 7 } & & $E$ & $G$ & & $E$ & $G$ \\
\hline FD1 & 0.691 & 0.47 & -0.07 & & 0.5 & -0.57 \\
FD2 & 0.62 & 0.54 & 0.03 & & 0.82 & -4.18 \\
FD3 & 0.578 & 0.58 & 0.09 & & 1.01 & -6.3 \\
FE0 & 0.831 & 0.35 & 0.01 & & 0.11 & 9.06 \\
FE1 & 0.719 & 0.56 & 0.02 & & 0.52 & 1.27 \\
FE2 & 0.672 & 0.65 & 0 & & 0.7 & -1.86 \\
FE3 & 0.644 & 0.71 & 0.05 & & 0.82 & -3.65 \\
\hline
\end{tabular}


Table G.4 Results of calculations for long specimens with $b / h=3$.

\begin{tabular}{|c|c|c|c|c|c|}
\hline \multirow[t]{2}{*}{$\begin{array}{l}\text { Material } \\
\text { ID }\end{array}$} & \multirow[t]{2}{*}{$\begin{array}{c}\text { Best } \\
\alpha\end{array}$} & \multicolumn{2}{|c|}{$\begin{array}{c}\begin{array}{c}\text { \%Error based on } \\
\text { the best } \alpha\end{array} \\
\end{array}$} & \multicolumn{2}{|c|}{$\begin{array}{c}\text { \%Error based on } \\
\alpha \alpha=0.7\end{array}$} \\
\hline & & $E$ & $G$ & $E$ & $G$ \\
\hline AAO & 0.869 & 0 & 0.1 & -0.47 & 5.54 \\
\hline AAl & 0.695 & 0.09 & 0 & 0.1 & -0.15 \\
\hline $\mathrm{AA} 2$ & 0.616 & 0.12 & -0.01 & 0.35 & -2.66 \\
\hline $\mathrm{AA} 3$ & 0.569 & 0.14 & -0.06 & 0.51 & -4.16 \\
\hline AB0 & 0.85 & 0.01 & 0.03 & -0.32 & 5.07 \\
\hline AB 1 & 0.681 & 0.09 & 0 & 0.14 & -0.61 \\
\hline $\mathrm{AB} 2$ & 0.606 & 0.13 & -0.06 & 0.34 & -3.12 \\
\hline AB3 & 0.559 & 0.14 & -0.06 & 0.46 & -4.62 \\
\hline $\mathrm{AC} 0$ & 0.827 & 0.02 & 0.01 & -0.19 & 4.55 \\
\hline $\mathrm{ACl}$ & 0.667 & 0.1 & 0.02 & 0.16 & -1.13 \\
\hline AC2 & 0.597 & 0.14 & -0.06 & 0.31 & -3.63 \\
\hline AC3 & 0.55 & 0.15 & 0.02 & 0.4 & -5.11 \\
\hline AD0 & 0.798 & 0.04 & 0.02 & -0.07 & 3.98 \\
\hline $\mathrm{AD} 1$ & 0.658 & 0.12 & -0.02 & 0.17 & -1.66 \\
\hline AD2 & 0.592 & 0.15 & 0.01 & 0.27 & -4.13 \\
\hline AD3 & 0.555 & 0.17 & -0.06 & 0.33 & -5.6 \\
\hline AE0 & 0.77 & 0.09 & -0.09 & 0.05 & 3.65 \\
\hline $\mathrm{AE} 1$ & 0.667 & 0.16 & -0.03 & 0.17 & -1.72 \\
\hline AE2 & 0.62 & 0.18 & 0.02 & 0.23 & -4.02 \\
\hline AE3 & 0.592 & 0.2 & 0.03 & 0.27 & -5.38 \\
\hline BA0 & 0.888 & 0.06 & -0.09 & -0.46 & 5.98 \\
\hline BA1 & 0.709 & 0.14 & -0.05 & 0.12 & 0.25 \\
\hline BA2 & 0.625 & 0.16 & 0.08 & 0.37 & -2.28 \\
\hline BA3 & 0.578 & 0.18 & 0.03 & 0.52 & -3.79 \\
\hline BB0 & 0.869 & 0.06 & -0.07 & -0.31 & 5.61 \\
\hline BB1 & 0.695 & 0.14 & 0.05 & 0.15 & -0.11 \\
\hline BB2 & 0.62 & 0.18 & -0.02 & 0.35 & -2.63 \\
\hline BB3 & 0.573 & 0.19 & -0.02 & 0.47 & -4.13 \\
\hline $\mathrm{BCO}$ & 0.845 & 0.07 & 0.04 & -0.18 & 5.26 \\
\hline $\mathrm{BCl}$ & 0.686 & 0.15 & 0.06 & 0.17 & -0.44 \\
\hline BC2 & 0.616 & 0.19 & -0.02 & 0.33 & -2.94 \\
\hline BC3 & 0.569 & 0.2 & 0.08 & 0.42 & -4.43 \\
\hline BD0 & 0.827 & 0.09 & -0.07 & -0.05 & 5.04 \\
\hline BDI & 0.686 & 0.17 & -0.03 & 0.19 & -0.58 \\
\hline BD2 & 0.62 & 0.2 & 0.05 & 0.29 & -3.03 \\
\hline BD3 & 0.583 & 0.22 & 0 & 0.36 & -4.5 \\
\hline BE0 & 0.808 & 0.14 & 0.1 & 0.08 & 5.9 \\
\hline
\end{tabular}


Table G.4 (Continued)

\begin{tabular}{|c|c|c|c|c|c|}
\hline \multirow[t]{2}{*}{$\begin{array}{l}\text { Material } \\
\text { ID }\end{array}$} & \multirow[t]{2}{*}{$\begin{array}{c}\text { Best } \\
\alpha\end{array}$} & \multicolumn{2}{|c|}{$\begin{array}{c}\text { \%Error based on } \\
\text { the best } \alpha\end{array}$} & \multicolumn{2}{|c|}{$\begin{array}{c}\text { \%Error based on } \\
\alpha=0.7\end{array}$} \\
\hline & & $E$ & $G$ & $E$ & $G$ \\
\hline $\mathrm{BEI}$ & 0.714 & 0.22 & -0.03 & 0.21 & 0.71 \\
\hline BE2 & 0.672 & 0.25 & -0.04 & 0.27 & -1.49 \\
\hline BE3 & 0.644 & 0.28 & 0.08 & 0.31 & -2.79 \\
\hline CAO & 0.897 & 0.1 & 0.04 & -0.45 & 6.41 \\
\hline $\mathrm{CAl}$ & 0.719 & 0.18 & 0.04 & 0.13 & 0.64 \\
\hline $\mathrm{CA} 2$ & 0.639 & 0.22 & 0.01 & 0.39 & -1.91 \\
\hline CA3 & 0.592 & 0.24 & -0.04 & 0.54 & -3.42 \\
\hline CBO & 0.883 & 0.11 & -0.03 & -0.3 & 6.14 \\
\hline CBI & 0.709 & 0.19 & 0.08 & 0.17 & 0.39 \\
\hline CB2 & 0.634 & 0.23 & 0.01 & 0.37 & -2.14 \\
\hline CB3 & 0.587 & 0.24 & 0.01 & 0.49 & -3.64 \\
\hline $\mathrm{CCO}$ & 0.864 & 0.12 & 0.06 & -0.16 & 5.96 \\
\hline $\mathrm{CCl}$ & 0.709 & 0.21 & -0.07 & 0.19 & 0.26 \\
\hline $\mathrm{CC} 2$ & 0.634 & 0.24 & 0.03 & 0.35 & -2.25 \\
\hline $\mathrm{CC} 3$ & 0.592 & 0.26 & -0.02 & 0.44 & -3.74 \\
\hline CDO & 0.85 & 0.14 & 0.04 & -0.03 & 6.12 \\
\hline $\mathrm{CD} 1$ & 0.714 & 0.23 & -0.03 & 0.21 & 0.53 \\
\hline CD2 & 0.653 & 0.27 & -0.08 & 0.32 & -1.91 \\
\hline CD3 & 0.611 & 0.28 & 0.07 & 0.38 & -3.36 \\
\hline CE0 & 0.85 & 0.2 & 0.07 & 0.11 & 8.24 \\
\hline CEl & 0.761 & 0.29 & 0.03 & 0.25 & 3.26 \\
\hline CE2 & 0.723 & 0.33 & -0.05 & 0.32 & 1.18 \\
\hline CE3 & 0.7 & 0.36 & -0.05 & 0.36 & -0.05 \\
\hline DAl & 0.705 & 0.1 & -0.04 & 0.09 & 0.11 \\
\hline DA2 & 0.625 & 0.13 & -0.04 & 0.34 & -2.39 \\
\hline DA3 & 0.578 & 0.15 & -0.07 & 0.49 & -3.89 \\
\hline DB0 & 0.859 & 0.02 & -0.04 & -0.34 & 5.32 \\
\hline DBI & 0.691 & 0.1 & -0.02 & 0.12 & -0.33 \\
\hline DB2 & 0.616 & 0.14 & -0.06 & 0.33 & -2.82 \\
\hline DB3 & 0.569 & 0.15 & -0.04 & 0.45 & -4.3 \\
\hline DCO & 0.836 & 0.03 & -0.05 & -0.2 & 4.82 \\
\hline $\mathrm{DCl}$ & 0.677 & 0.11 & 0.02 & 0.15 & -0.8 \\
\hline DC2 & 0.606 & 0.14 & -0.02 & 0.3 & -3.26 \\
\hline DC3 & 0.559 & 0.16 & 0.09 & 0.39 & -4.73 \\
\hline DD0 & 0.808 & 0.05 & -0.03 & -0.07 & 4.31 \\
\hline DDI & 0.667 & 0.13 & 0.05 & 0.16 & -1.23 \\
\hline DD2 & 0.606 & 0.16 & -0.02 & 0.27 & -3.64 \\
\hline DD3 & 0.569 & 0.18 & -0.05 & 0.33 & -5.07 \\
\hline
\end{tabular}


Table G.4 (Continued)

\begin{tabular}{|c|c|c|c|c|c|}
\hline \multirow[t]{2}{*}{$\begin{array}{l}\text { Material } \\
\text { ID }\end{array}$} & \multirow[t]{2}{*}{$\begin{array}{c}\text { Best } \\
\alpha\end{array}$} & \multicolumn{2}{|c|}{$\begin{array}{c}\text { \%Error based on } \\
\text { the best } \alpha\end{array}$} & \multicolumn{2}{|c|}{$\begin{array}{c}\text { \%Error based on } \\
\alpha=0.7\end{array}$} \\
\hline & & $E$ & $G$ & $E$ & $G$ \\
\hline DE0 & 0.78 & 0.09 & -0.08 & 0.04 & 4.18 \\
\hline DEl & 0.681 & 0.16 & 0.01 & 0.18 & -0.96 \\
\hline DE2 & 0.639 & 0.2 & 0.01 & 0.23 & -3.09 \\
\hline DE3 & 0.616 & 0.22 & -0.07 & 0.27 & -4.34 \\
\hline EA0 & 0.892 & 0.05 & 0.05 & -0.48 & 6.27 \\
\hline EAI & 0.719 & 0.15 & -0.03 & 0.1 & 0.57 \\
\hline EA2 & 0.639 & 0.18 & -0.02 & 0.35 & -1.94 \\
\hline EA3 & 0.587 & 0.19 & 0.08 & 0.5 & -3.45 \\
\hline EB0 & 0.878 & 0.07 & -0.09 & -0.33 & 5.92 \\
\hline EB1 & 0.709 & 0.16 & -0.06 & 0.14 & 0.25 \\
\hline EB2 & 0.634 & 0.19 & -0.1 & 0.34 & -2.25 \\
\hline EB3 & 0.587 & 0.21 & -0.08 & 0.46 & -3.74 \\
\hline EC0 & 0.855 & 0.07 & 0.04 & -0.19 & 5.6 \\
\hline $\mathrm{ECl}$ & 0.7 & 0.16 & -0.02 & 0.16 & -0.02 \\
\hline EC2 & 0.63 & 0.2 & -0.04 & 0.32 & -2.48 \\
\hline EC3 & 0.587 & 0.22 & -0.07 & 0.41 & -3.95 \\
\hline ED0 & 0.836 & 0.1 & -0.04 & -0.06 & 5.46 \\
\hline ED1 & 0.7 & 0.18 & -0.04 & 0.18 & -0.04 \\
\hline ED2 & 0.639 & 0.22 & -0.05 & 0.29 & -2.42 \\
\hline ED3 & 0.602 & 0.24 & -0.04 & 0.35 & -3.83 \\
\hline EE0 & 0.822 & 0.15 & -0.03 & 0.07 & 6.56 \\
\hline EE I & 0.733 & 0.23 & -0.06 & 0.21 & 1.66 \\
\hline EE2 & 0.695 & 0.27 & -0.08 & 0.28 & -0.33 \\
\hline EE3 & 0.672 & 0.3 & -0.02 & 0.32 & -1.47 \\
\hline FAO & 0.906 & 0.1 & 0.08 & -0.48 & 6.75 \\
\hline FAl & 0.733 & 0.2 & -0.02 & 0.11 & 1.02 \\
\hline FA2 & 0.653 & 0.24 & -0.02 & 0.37 & -1.5 \\
\hline FA3 & 0.606 & 0.26 & -0.06 & 0.52 & -3.01 \\
\hline FB0 & 0.892 & 0.11 & 0.03 & -0.32 & 6.52 \\
\hline FBI & 0.723 & 0.2 & 0.05 & 0.15 & 0.83 \\
\hline FB2 & 0.648 & 0.24 & 0.01 & 0.36 & -1.68 \\
\hline FB3 & 0.602 & 0.26 & 0.04 & 0.48 & -3.17 \\
\hline $\mathrm{FC} 0$ & 0.878 & 0.13 & -0.04 & -0.17 & 6.39 \\
\hline $\mathrm{FCl}$ & 0.723 & 0.22 & -0.06 & 0.18 & 0.76 \\
\hline FC2 & 0.653 & 0.26 & -0.06 & 0.34 & -1.69 \\
\hline FC3 & 0.606 & 0.27 & 0.08 & 0.43 & -3.16 \\
\hline FD0 & 0.864 & 0.15 & -0.04 & -0.04 & 6.63 \\
\hline FDl & 0.728 & 0.24 & 0.08 & 0.21 & 1.19 \\
\hline
\end{tabular}


Table G.4 (Continued)

\begin{tabular}{ccccccc}
\hline Material & Best & \multicolumn{2}{c}{$\begin{array}{c}\text { \%Error based on } \\
\text { ID }\end{array}$} & $\alpha$ & & \multicolumn{2}{c}{$\begin{array}{c}\text { \%Error based on } \\
\alpha=0.7\end{array}$} \\
\cline { 3 - 4 } \cline { 6 - 7 } & & $E$ & $G$ & & $E$ & $G$ \\
\hline FD2 & 0.672 & 0.28 & -0.06 & & 0.32 & -1.16 \\
FD3 & 0.634 & 0.31 & 0 & & 0.38 & -2.55 \\
FE0 & 0.864 & 0.21 & 0.07 & & 0.11 & 9.04 \\
FE1 & 0.784 & 0.3 & -0.08 & & 0.26 & 4.43 \\
FE2 & 0.749 & 0.36 & 0.01 & & 0.33 & 2.61 \\
FE3 & 0.73 & 0.39 & -0.01 & & 0.37 & 1.59 \\
\hline
\end{tabular}


Table G.5 Results of calculations for short specimens with $b / h=5$.

\begin{tabular}{|c|c|c|c|c|c|}
\hline \multirow[t]{2}{*}{$\begin{array}{c}\text { Material } \\
\text { ID }\end{array}$} & \multirow[t]{2}{*}{$\begin{array}{c}\text { Best } \\
\alpha\end{array}$} & \multicolumn{2}{|c|}{$\begin{array}{l}\text { \%Error based on } \\
\text { the best } \alpha\end{array}$} & \multicolumn{2}{|c|}{$\begin{array}{c}\begin{array}{c}\% \text { Error based on } \\
\alpha=0.7\end{array} \\
\end{array}$} \\
\hline & & $E$ & $G$ & $E$ & $G$ \\
\hline AA0 & 0.845 & 0.01 & 0 & -1.23 & 6.7 \\
\hline AAl & 0.667 & 0.27 & -0.03 & 0.55 & -1.53 \\
\hline $\mathrm{AA} 2$ & 0.587 & 0.37 & -0.01 & 1.33 & -5.09 \\
\hline AA3 & $0.54 !$ & 0.43 & -0.02 & 1.79 & -7.2 \\
\hline $\mathrm{AB} 0$ & 0.827 & 0.04 & -0.08 & -0.83 & 5.94 \\
\hline $\mathrm{AB} 1$ & 0.653 & 0.29 & -0.08 & 0.61 & -2.27 \\
\hline $\mathrm{AB} 2$ & 0.573 & 0.37 & 0.05 & 1.24 & -5.82 \\
\hline AB3 & 0.531 & 0.45 & -0.1 & 1.61 & -7.9 \\
\hline AC0 & 0.801 & 0.06 & 0 & -0.46 & 5.01 \\
\hline $\mathrm{ACl}$ & 0.634 & 0.3 & 0.02 & 0.64 & -3.18 \\
\hline AC2 & 0.562 & 0.41 & 0 & 1.13 & -6.68 \\
\hline AC3 & 0.517 & 0.47 & 0.06 & 1.41 & -8.73 \\
\hline $\mathrm{ADO}$ & 0.77 & 0.12 & 0.03 & -0.12 & 3.83 \\
\hline $\mathrm{ADl}$ & 0.62 & 0.38 & -0.1 & 0.66 & -4.31 \\
\hline $\mathrm{AD} 2$ & 0.552 & 0.49 & 0 & 1 & -7.72 \\
\hline AD3 & 0.513 & 0.56 & 0.05 & 1.21 & -9.68 \\
\hline $\mathrm{AE} 0$ & 0.733 & 0.27 & 0.03 & 0.22 & 2.24 \\
\hline $\mathrm{AE} 1$ & 0.616 & 0.56 & -0.06 & 0.71 & -5.58 \\
\hline AE2 & 0.569 & 0.71 & -0.08 & 0.95 & -8.55 \\
\hline AE3 & 0.541 & 0.81 & 0.07 & 1.1 & -10.12 \\
\hline BA0 & 0.85 & 0.09 & 0.02 & -1.19 & 6.94 \\
\hline BAl & 0.672 & 0.39 & -0.02 & 0.63 & -1.3 \\
\hline $\mathrm{BA} 2$ & 0.592 & 0.5 & 0.03 & 1.42 & -4.85 \\
\hline BA3 & 0.545 & 0.56 & 0.04 & 1.88 & -6.93 \\
\hline BB0 & 0.831 & 0.11 & 0.03 & -0.79 & 6.26 \\
\hline BBI & 0.658 & 0.4 & 0.02 & 0.69 & -1.95 \\
\hline BB2 & 0.583 & 0.53 & -0.02 & 1.33 & -5.46 \\
\hline BB3 & 0.536 & 0.58 & 0.06 & 1.71 & -7.52 \\
\hline $\mathrm{BC} 0$ & 0.81 & 0.16 & 0 & -0.41 & 5.49 \\
\hline $\mathrm{BCl}$ & 0.644 & 0.44 & 0.06 & 0.73 & -2.68 \\
\hline $\mathrm{BC} 2$ & 0.573 & 0.57 & -0.02 & 1.23 & -6.14 \\
\hline $\mathrm{BC} 3$ & 0.531 & 0.65 & -0.02 & 1.52 & -8.15 \\
\hline BD0 & 0.784 & 0.24 & 0.04 & -0.05 & 4.62 \\
\hline BDI & 0.634 & 0.53 & 0.03 & 0.76 & -3.45 \\
\hline BD2 & 0.571 & 0.68 & -0.01 & 1.13 & -6.77 \\
\hline BD3 & 0.534 & 0.77 & 0.01 & 1.35 & -8.66 \\
\hline BE0 & 0.761 & 0.44 & -0.08 & 0.33 & 4.05 \\
\hline
\end{tabular}


Table G.5 (Continued)

\begin{tabular}{|c|c|c|c|c|c|}
\hline \multirow[t]{2}{*}{$\begin{array}{l}\text { Material } \\
\text { ID }\end{array}$} & \multirow[t]{2}{*}{$\begin{array}{c}\text { Best } \\
\alpha\end{array}$} & \multicolumn{2}{|c|}{$\begin{array}{c}\text { \%Error based on } \\
\text { the best } \alpha\end{array}$} & \multicolumn{2}{|c|}{$\begin{array}{c}\text { \%Error based on } \\
\alpha=0.7\end{array}$} \\
\hline & & $E$ & $G$ & $E$ & $G$ \\
\hline BEl & 0.648 & 0.79 & -0.03 & 0.88 & -3.43 \\
\hline BE2 & 0.606 & 0.99 & -0.02 & 1.16 & -6.12 \\
\hline BE3 & 0.585 & 1.13 & -0.03 & 1.33 & -7.45 \\
\hline $\mathrm{CA} 0$ & 0.855 & 0.17 & 0.03 & -1.15 & 7.17 \\
\hline CAl & 0.677 & 0.51 & -0.03 & 0.71 & -1.09 \\
\hline CA2 & 0.597 & 0.63 & 0.04 & 1.51 & -4.62 \\
\hline $\mathrm{CA}_{3}$ & 0.55 & 0.7 & 0.07 & 1.98 & -6.68 \\
\hline CB0 & 0.838 & 0.21 & 0.02 & -0.74 & 6.58 \\
\hline CBI & 0.665 & 0.53 & 0.01 & 0.77 & -1.64 \\
\hline CB2 & 0.587 & 0.66 & 0.1 & 1.43 & -5.12 \\
\hline CB3 & 0.545 & 0.76 & 0 & 1.81 & -7.15 \\
\hline $\mathrm{CC} 0$ & 0.82 & 0.27 & 0.01 & -0.35 & 5.96 \\
\hline $\mathrm{CCl}$ & 0.653 & 0.58 & 0.1 & 0.83 & -2.19 \\
\hline $\mathrm{CC} 2$ & 0.583 & 0.74 & 0.08 & 1.34 & -5.6 \\
\hline $\mathrm{CC} 3$ & 0.545 & 0.85 & -0.09 & 1.65 & -7.56 \\
\hline CD0 & 0.798 & 0.37 & 0.07 & 0.02 & 5.42 \\
\hline CD1 & 0.653 & 0.72 & -0.07 & 0.88 & -2.56 \\
\hline CD2 & 0.59 & 0.89 & 0.01 & 1.27 & -5.78 \\
\hline CD3 & 0.555 & 1 & 0.01 & 1.51 & -7.58 \\
\hline CE0 & 0.787 & 0.62 & 0.06 & 0.47 & 5.96 \\
\hline CEl & 0.684 & 1.06 & -0.05 & 1.09 & -1.14 \\
\hline CE2 & 0.646 & 1.31 & 0.04 & 1.4 & -3.5 \\
\hline CE3 & 0.63 & 1.49 & 0.03 & 1.62 & -4.56 \\
\hline DA0 & 0.845 & 0.01 & 0.04 & -1.22 & 6.73 \\
\hline DAl & 0.667 & 0.28 & 0.05 & 0.56 & -1.45 \\
\hline DA2 & 0.59 & 0.4 & 0 & 1.34 & -4.98 \\
\hline DA3 & 0.545 & 0.48 & -0.09 & 1.8 & -7.06 \\
\hline DB0 & 0.827 & 0.04 & -0.03 & -0.83 & 5.98 \\
\hline DB 1 & 0.653 & 0.3 & 0.01 & 0.62 & -2.18 \\
\hline DB2 & 0.578 & 0.42 & -0.03 & 1.25 & -5.69 \\
\hline DB3 & 0.531 & 0.47 & 0.05 & 1.63 & -7.74 \\
\hline DCO & 0.803 & 0.08 & -0.07 & -0.45 & 5.06 \\
\hline $\mathrm{DCl}$ & 0.639 & 0.34 & -0.09 & 0.65 & -3.07 \\
\hline DC2 & 0.564 & 0.44 & 0.05 & 1.14 & -6.52 \\
\hline DC3 & 0.522 & 0.52 & 0.04 & 1.44 & -8.52 \\
\hline DD0 & 0.773 & 0.14 & -0.03 & -0.11 & 3.91 \\
\hline DDI & 0.62 & 0.4 & 0.05 & 0.67 & -4.16 \\
\hline DD2 & 0.557 & 0.53 & -0.01 & 1.03 & -7.49 \\
\hline
\end{tabular}


Table G.5 (Continued)

\begin{tabular}{|c|c|c|c|c|c|}
\hline \multirow[t]{2}{*}{$\begin{array}{l}\text { Material } \\
\text { ID }\end{array}$} & \multirow[t]{2}{*}{$\begin{array}{c}\text { Best } \\
\alpha\end{array}$} & \multicolumn{2}{|c|}{$\begin{array}{l}\% \text { Error based on } \\
\text { the best } \alpha\end{array}$} & \multicolumn{2}{|c|}{$\begin{array}{c}\% \text { Error based on } \\
\alpha=0.7\end{array}$} \\
\hline & & $E$ & $G$ & $E$ & $G$ \\
\hline DD3 & 0.52 & 0.61 & 0 & 1.24 & -9.38 \\
\hline DE0 & 0.735 & 0.29 & 0 & 0.23 & 2.37 \\
\hline DEl & 0.62 & 0.59 & -0.06 & 0.73 & -5.28 \\
\hline DE2 & 0.573 & 0.76 & 0.09 & 0.98 & -8.07 \\
\hline DE3 & 0.552 & 0.88 & 0 & 1.15 & -9.47 \\
\hline EA0 & 0.85 & 0.09 & 0.07 & -1.18 & 6.99 \\
\hline EAl & 0.672 & 0.4 & 0.08 & 0.64 & -1.2 \\
\hline EA2 & 0.597 & 0.55 & -0.04 & 1.43 & -4.71 \\
\hline EA3 & 0.55 & 0.62 & 0 & 1.9 & -6.76 \\
\hline EB0 & 0.831 & 0.12 & 0.09 & -0.78 & 6.32 \\
\hline EB1 & 0.663 & 0.44 & -0.08 & 0.7 & -1.84 \\
\hline EB2 & 0.587 & 0.57 & -0.07 & 1.34 & -5.3 \\
\hline EB3 & 0.541 & 0.64 & 0.05 & 1.73 & -7.32 \\
\hline EC0 & 0.813 & 0.18 & -0.05 & -0.4 & 5.55 \\
\hline $\mathrm{ECl}$ & 0.648 & 0.48 & -0.03 & 0.74 & -2.54 \\
\hline EC2 & 0.578 & 0.62 & -0.03 & 1.25 & -5.94 \\
\hline EC3 & 0.536 & 0.7 & 0.01 & 1.55 & -7.89 \\
\hline ED0 & 0.787 & 0.26 & 0 & -0.04 & 4.71 \\
\hline EDI & 0.639 & 0.57 & -0.02 & 0.78 & -3.25 \\
\hline ED2 & 0.578 & 0.73 & -0.08 & 1.16 & -6.48 \\
\hline ED3 & 0.541 & 0.84 & 0.03 & 1.39 & -8.27 \\
\hline EE0 & 0.761 & 0.46 & 0.09 & 0.35 & 4.22 \\
\hline EEl & 0.653 & 0.83 & 0.04 & 0.92 & -3.05 \\
\hline EE2 & 0.616 & 1.06 & 0 & 1.21 & -5.5 \\
\hline EE3 & 0.597 & 1.22 & 0.08 & 1.41 & -6.61 \\
\hline FA0 & 0.855 & 0.18 & 0.1 & -1.14 & 7.22 \\
\hline FAl & 0.677 & 0.52 & 0.09 & 0.72 & -0.97 \\
\hline FA2 & 0.602 & 0.69 & 0 & 1.53 & -4.45 \\
\hline FA3 & 0.555 & 0.77 & 0.07 & 2 & -6.47 \\
\hline FB0 & 0.841 & 0.23 & -0.03 & -0.73 & 6.65 \\
\hline FB1 & 0.667 & 0.57 & 0.03 & 0.79 & -1.5 \\
\hline FB2 & 0.592 & 0.71 & 0.08 & 1.45 & -4.92 \\
\hline FB3 & 0.55 & 0.82 & 0.03 & 1.84 & -6.9 \\
\hline $\mathrm{FC} 0$ & 0.822 & 0.29 & -0.03 & -0.34 & 6.05 \\
\hline $\mathrm{FCl}$ & 0.658 & 0.63 & 0.04 & 0.84 & -2.02 \\
\hline FC2 & 0.587 & 0.79 & 0.1 & 1.37 & -5.35 \\
\hline $\mathrm{FC} 3$ & 0.55 & 0.91 & -0.01 & 1.68 & -7.24 \\
\hline FD0 & 0.803 & 0.4 & -0.08 & 0.04 & 5.54 \\
\hline
\end{tabular}


Table G.5 (Continued)

\begin{tabular}{ccccccc}
\hline $\begin{array}{c}\text { Material } \\
\text { ID }\end{array}$ & $\begin{array}{c}\text { Best } \\
\alpha\end{array}$ & \multicolumn{2}{c}{$\begin{array}{c}\text { \%Error based on } \\
\text { the best } \alpha\end{array}$} & \multicolumn{2}{c}{$\begin{array}{c}\text { \%Error based on } \\
\alpha=0.7\end{array}$} \\
\cline { 3 - 4 } \cline { 5 - 6 } & & $E$ & $G$ & & $E$ & $G$ \\
\hline FD1 & 0.658 & 0.76 & -0.08 & & 0.91 & -2.32 \\
FD2 & 0.597 & 0.95 & 0 & & 1.31 & -5.42 \\
FD3 & 0.564 & 1.09 & 0 & & 1.56 & -7.11 \\
FE0 & 0.791 & 0.65 & -0.07 & & 0.49 & 6.17 \\
FE1 & 0.691 & 1.12 & -0.04 & & 1.13 & -0.66 \\
FE2 & 0.658 & 1.4 & 0.05 & & 1.48 & -2.73 \\
FE3 & 0.646 & 1.62 & 0.03 & & 1.72 & -3.5 \\
\hline
\end{tabular}


Table G. 6 Results of calculations for long specimens with $b / h=5$.

\begin{tabular}{|c|c|c|c|c|c|}
\hline \multirow[t]{2}{*}{$\begin{array}{l}\text { Material } \\
\text { ID }\end{array}$} & \multirow[t]{2}{*}{$\begin{array}{c}\text { Best } \\
\alpha\end{array}$} & \multicolumn{2}{|c|}{$\begin{array}{l}\% \text { Error based on } \\
\text { the best } \alpha\end{array}$} & \multicolumn{2}{|c|}{$\begin{array}{c}\text { \%Error based on } \\
\alpha=0.7\end{array}$} \\
\hline & & $E$ & $G$ & $E$ & $G$ \\
\hline$\overline{\mathrm{AA} 0}$ & 0.841 & 0.04 & 0.03 & -0.35 & 4.55 \\
\hline AAl & 0.677 & 0.16 & -0.02 & 0.23 & -0.76 \\
\hline AA2 & 0.602 & 0.21 & 0.03 & 0.48 & -3.05 \\
\hline $\mathrm{AA} 3$ & 0.559 & 0.24 & -0.02 & 0.63 & -4.41 \\
\hline $\mathrm{AB} 0$ & 0.822 & 0.06 & -0.05 & -0.21 & 4.04 \\
\hline $\mathrm{AB} 1$ & 0.663 & 0.18 & -0.01 & 0.26 & -1.24 \\
\hline $\mathrm{AB} 2$ & 0.592 & 0.23 & 0 & 0.47 & -3.51 \\
\hline $\mathrm{AB} 3$ & 0.55 & 0.26 & 0.01 & 0.59 & -4.84 \\
\hline $\mathrm{ACO}$ & 0.798 & 0.09 & -0.07 & -0.08 & 3.44 \\
\hline $\mathrm{ACl}$ & 0.648 & 0.2 & 0.02 & 0.29 & -1.78 \\
\hline $\mathrm{AC} 2$ & 0.583 & 0.25 & 0.05 & 0.45 & -3.98 \\
\hline $\mathrm{AC} 3$ & 0.545 & 0.29 & 0.02 & 0.55 & -5.27 \\
\hline $\mathrm{ADO}$ & 0.77 & 0.13 & -0.05 & 0.05 & 2.76 \\
\hline ADl & 0.639 & 0.25 & 0.06 & 0.32 & -2.31 \\
\hline $\mathrm{AD} 2$ & 0.587 & 0.31 & -0.05. & 0.44 & -4.36 \\
\hline AD3 & 0.555 & 0.35 & 0 & 0.52 & -5.53 \\
\hline $\mathrm{AEO}$ & 0.742 & 0.23 & 0.01 & 0.2 & 2.23 \\
\hline $\mathrm{AE} 1$ & 0.663 & 0.38 & -0.09 & 0.4 & -2.02 \\
\hline AE2 & 0.634 & 0.46 & -0.04 & 0.5 & -3.38 \\
\hline AE3 & 0.62 & 0.52 & 0.05 & 0.57 & -3.98 \\
\hline BAO & 0.85 & 0.1 & -0.04 & -0.31 & 4.79 \\
\hline BAl & 0.686 & 0.24 & -0.03 & 0.28 & -0.47 \\
\hline $\mathrm{BA} 2$ & 0.616 & 0.3 & -0.08 & 0.53 & -2.73 \\
\hline $\mathrm{BA3}$ & 0.569 & 0.32 & 0.04 & 0.68 & -4.06 \\
\hline BBO & 0.831 & 0.12 & -0.03 & -0.17 & 4.37 \\
\hline BB 1 & 0.672 & 0.25 & 0.08 & 0.31 & -0.84 \\
\hline BB2 & 0.606 & 0.31 & -0.01 & 0.52 & -3.06 \\
\hline BB3 & 0.564 & 0.35 & 0.04 & 0.65 & -4.36 \\
\hline $\mathrm{BC} 0$ & 0.813 & 0.15 & -0.08 & -0.04 & 3.94 \\
\hline $\mathrm{BCl}$ & 0.667 & 0.29 & -0.04 & 0.35 & -1.18 \\
\hline $\mathrm{BC} 2$ & 0.606 & 0.36 & -0.08 & 0.52 & -3.32 \\
\hline$B C 3$ & 0.569 & 0.4 & -0.04 & 0.62 & -4.55 \\
\hline BDO & 0.789 & 0.2 & 0.04 & 0.1 & 3.61 \\
\hline BDI & 0.667 & 0.35 & 0 & 0.39 & -1.28 \\
\hline BD2 & 0.616 & 0.43 & 0.05 & 0.53 & -3.2 \\
\hline $\mathrm{BD3}$ & 0.587 & 0.48 & 0.04 & 0.61 & -4.27 \\
\hline BEO & 0.78 & 0.34 & 0.06 & 0.29 & 4.31 \\
\hline
\end{tabular}


Table G.6 (Continued)

\begin{tabular}{|c|c|c|c|c|c|}
\hline \multirow[t]{2}{*}{$\begin{array}{c}\text { Material } \\
\text { ID }\end{array}$} & \multirow[t]{2}{*}{$\begin{array}{c}\text { Best } \\
\alpha\end{array}$} & \multicolumn{2}{|c|}{$\begin{array}{l}\% \text { Error based on } \\
\text { the best } \alpha\end{array}$} & \multicolumn{2}{|c|}{$\begin{array}{c}\text { \%Error based on } \\
\alpha=0.7\end{array}$} \\
\hline & & $E$ & $G$ & $E$ & $G$ \\
\hline BEI & 0.712 & 0.53 & 0.01 & 0.52 & 0.63 \\
\hline BE2 & 0.695 & 0.65 & -0.07 & 0.65 & -0.31 \\
\hline BE3 & 0.691 & 0.73 & -0.09 & 0.74 & -0.57 \\
\hline CAO & 0.855 & 0.16 & 0.03 & -0.27 & 5.02 \\
\hline $\mathrm{CAl}$ & 0.691 & 0.3 & 0.1 & 0.33 & -0.2 \\
\hline $\mathrm{CA} 2$ & 0.625 & 0.38 & -0.06 & 0.59 & -2.42 \\
\hline $\mathrm{CA} 3$ & 0.578 & 0.41 & 0.09 & 0.75 & -3.72 \\
\hline CBO & 0.841 & 0.19 & -0.02 & -0.13 & 4.7 \\
\hline CB 1 & 0.686 & 0.34 & 0.01 & 0.37 & -0.45 \\
\hline $\mathrm{CB} 2$ & 0.62 & 0.41 & -0.01 & 0.59 & -2.61 \\
\hline CB3 & 0.578 & 0.45 & 0.08 & 0.72 & -3.87 \\
\hline $\mathrm{CCO}$ & 0.822 & 0.22 & 0.08 & 0.01 & 4.44 \\
\hline $\mathrm{CCl}$ & 0.681 & 0.38 & 0.08 & 0.41 & -0.58 \\
\hline CC2 & 0.625 & 0.46 & -0.03 & 0.59 & -2.63 \\
\hline $\mathrm{CC} 3$ & 0.587 & 0.51 & 0.07 & 0.7 & -3.8 \\
\hline CD0 & 0.813 & 0.29 & -0.05 & 0.16 & 4.48 \\
\hline $\mathrm{CD} 1$ & 0.695 & 0.47 & -0.02 & 0.48 & -0.2 \\
\hline $\mathrm{CD} 2$ & 0.648 & 0.57 & 0.02 & 0.63 & -1.98 \\
\hline CD3 & 0.625 & 0.64 & -0.03 & 0.72 & -2.93 \\
\hline CE0 & 0.822 & 0.47 & -0.07 & 0.39 & 6.51 \\
\hline $\mathrm{CE}_{1}$ & 0.766 & 0.71 & 0.01 & 0.67 & 3.48 \\
\hline CE2 & 0.756 & 0.86 & 0.08 & 0.83 & 3.04 \\
\hline CE3 & 0.761 & 0.98 & -0.04 & 0.94 & 3.18 \\
\hline DA0 & 0.841 & 0.04 & 0.08 & -0.35 & 4.6 \\
\hline DAl & 0.681 & 0.18 & -0.07 & 0.23 & -0.67 \\
\hline DA2 & 0.606 & 0.22 & 0.01 & 0.48 & -2.93 \\
\hline DA3 & 0.564 & 0.26 & -0.01 & 0.64 & -4.26 \\
\hline DB0 & 0.822 & 0.06 & 0.01 & -0.21 & 4.09 \\
\hline DBI & 0.663 & 0.18 & 0.09 & 0.26 & -1.13 \\
\hline DB2 & 0.597 & 0.24 & 0 & 0.47 & -3.36 \\
\hline DB3 & 0.559 & 0.28 & -0.1 & 0.6 & -4.65 \\
\hline DC0 & 0.798 & 0.09 & 0 & -0.08 & 3.51 \\
\hline $\mathrm{DCl}$ & 0.653 & 0.21 & -0.01 & 0.29 & -1.64 \\
\hline DC2 & 0.587 & 0.27 & 0.08 & 0.46 & -3.79 \\
\hline DC3 & 0.555 & 0.31 & -0.05 & 0.56 & -5.03 \\
\hline DD0 & 0.77 & 0.13 & 0.04 & 0.05 & 2.85 \\
\hline DDI & 0.644 & 0.26 & 0.07 & 0.33 & -2.12 \\
\hline DD2 & 0.592 & 0.33 & 0.06 & 0.45 & -4.08 \\
\hline
\end{tabular}


Table G.6 (Continued)

\begin{tabular}{|c|c|c|c|c|c|}
\hline \multirow[t]{2}{*}{$\begin{array}{l}\text { Material } \\
\text { ID }\end{array}$} & \multirow[t]{2}{*}{$\begin{array}{c}\text { Best } \\
\alpha\end{array}$} & \multicolumn{2}{|c|}{$\begin{array}{l}\% \text { Error based on } \\
\text { the best } \alpha\end{array}$} & \multicolumn{2}{|c|}{$\begin{array}{c}\text { \%Error based on } \\
\alpha=0.7\end{array}$} \\
\hline & & $E$ & $G$ & $E$ & $G$ \\
\hline DD3 & 0.564 & 0.38 & 0.02 & 0.53 & -5.16 \\
\hline DE0 & 0.747 & 0.24 & -0.07 & 0.21 & 2.41 \\
\hline DE1 & 0.667 & 0.4 & 0.07 & 0.42 & -1.62 \\
\hline DE2 & 0.646 & 0.49 & 0 & 0.53 & -2.75 \\
\hline DE3 & 0.639 & 0.57 & -0.02 & 0.6 & -3.13 \\
\hline EA0 & 0.85 & 0.11 & 0.02 & -0.31 & 4.85 \\
\hline EAl & 0.691 & 0.25 & -0.06 & 0.28 & -0.36 \\
\hline EA2 & 0.616 & 0.3 & 0.07 & 0.54 & -2.58 \\
\hline EA3 & 0.578 & 0.35 & -0.06 & 0.69 & -3.87 \\
\hline EB0 & 0.831 & 0.12 & 0.04 & -0.17 & 4.44 \\
\hline EB1 & 0.681 & 0.28 & -0.09 & 0.32 & -0.71 \\
\hline EB2 & 0.611 & 0.33 & 0.03 & 0.53 & -2.87 \\
\hline EB3 & 0.573 & 0.38 & -0.02 & 0.66 & -4.13 \\
\hline EC0 & 0.813 & 0.16 & 0.01 & -0.03 & 4.02 \\
\hline $\mathrm{ECl}$ & 0.672 & 0.31 & -0.03 & 0.35 & -1.02 \\
\hline EC2 & 0.611 & 0.38 & 0 & 0.53 & -3.08 \\
\hline EC3 & 0.578 & 0.43 & -0.05 & 0.64 & -4.24 \\
\hline ED0 & 0.794 & 0.21 & -0.04 & 0.11 & 3.72 \\
\hline EDI & 0.672 & 0.37 & 0.06 & 0.4 & -1.04 \\
\hline ED2 & 0.625 & 0.46 & 0.06 & 0.54 & -2.84 \\
\hline ED3 & 0.602 & 0.52 & -0.01 & 0.64 & -3.8 \\
\hline EE0 & 0.784 & 0.35 & 0.02 & 0.3 & 4.53 \\
\hline EE1 & 0.723 & 0.56 & -0.09 & 0.55 & 1.14 \\
\hline EE2 & 0.709 & 0.69 & 0.03 & 0.69 & 0.52 \\
\hline EE3 & 0.709 & 0.79 & 0.07 & 0.79 & 0.56 \\
\hline FA0 & 0.859 & 0.17 & -0.05 & -0.27 & 5.09 \\
\hline FAl & 0.7 & 0.34 & -0.06 & 0.34 & -0.06 \\
\hline FA2 & 0.63 & 0.4 & -0.02 & 0.6 & -2.23 \\
\hline FA3 & 0.587 & 0.45 & 0.03 & 0.76 & -3.49 \\
\hline FBO & 0.841 & 0.19 & 0.06 & -0.12 & 4.78 \\
\hline FB 1 & 0.691 & 0.36 & 0.02 & 0.38 & -0.29 \\
\hline FB2 & 0.625 & 0.43 & 0.06 & 0.6 & -2.39 \\
\hline FB3 & 0.587 & 0.48 & 0.06 & 0.74 & -3.59 \\
\hline $\mathrm{FC} 0$ & 0.827 & 0.23 & 0.01 & 0.02 & 4.54 \\
\hline $\mathrm{FCl}$ & 0.691 & 0.41 & -0.05 & 0.42 & -0.37 \\
\hline FC2 & 0.634 & 0.5 & -0.06 & 0.61 & -2.34 \\
\hline $\mathrm{FC} 3$ & 0.602 & 0.56 & -0.03 & 0.72 & -3.43 \\
\hline FD0 & 0.813 & 0.3 & 0.09 & 0.17 & 4.62 \\
\hline
\end{tabular}


Table G.6 (Continued)

\begin{tabular}{ccccccc}
\hline $\begin{array}{c}\text { Material } \\
\text { ID }\end{array}$ & $\begin{array}{c}\text { Best } \\
\alpha\end{array}$ & \multicolumn{2}{c}{$\begin{array}{c}\text { \%Error based on } \\
\text { the best } \alpha\end{array}$} & & \multicolumn{2}{c}{$\begin{array}{c}\text { \%Error based on } \\
\alpha=0.7\end{array}$} \\
\cline { 3 - 4 } \cline { 6 - 7 } \cline { 6 - 7 } & & $E$ & $G$ & & $E$ & $G$ \\
\hline FD1 & 0.7 & 0.49 & 0.1 & & 0.49 & 0.1 \\
FD2 & 0.663 & 0.61 & -0.07 & & 0.65 & -1.53 \\
FD3 & 0.639 & 0.69 & 0.02 & & 0.75 & -2.35 \\
FE0 & 0.827 & 0.48 & -0.06 & & 0.41 & 6.78 \\
FE1 & 0.777 & 0.75 & 0.02 & & 0.7 & 4.13 \\
FE2 & 0.777 & 0.92 & -0.02 & & 0.88 & 4.09 \\
FE3 & 0.787 & 1.06 & 0 & & 1.01 & 4.61 \\
\hline
\end{tabular}




\section{Appendix H}

\section{THE VSM APPARATUS}

Figure $\mathrm{H}-1$ illustrates the apparatus developed and used for the VSM. Each component of the apparatus is identified by a number. As the elevation of the apparatus shows, it comprises of a base (4), two supporting blocks (3), a middle assembly composed of parts (5) through (13), two side assemblies composed of parts (14) through (20) and two connecting bridges (21).

The test specimen (1) sits on two supporting rollers (2) parallel to each other. The rollers (2) are located on the support blocks (3), and the support blocks (3) rest on the base (4). By sliding the support blocks (3) over the base (4) one can change the span of the specimen (1).

Part (5) is a loading nose which is connected to the top plate (6) and is parallel to the supporting rollers (2). The top plate is rigidly connected to the load frame by a screw between the threaded hole (7) and the load frame. By relative vertical movement of the base (4) with respect to the top plate (6), load is applied to the specimen (1) through the support rollers (2) and the loading nose (5).

A locating beam (8) passes underneath the test specimen (1). The contact point (9) on the top of the locating beam (8) makes contact to the bottom of the specimen (1) at the center of the loading nose (5). The locating beam is connected to two guide bars (10) which restrict the movements of the locating beam, except in the vertical direction. The two guide bars (10) slide through two holes provided in the top plate (6). The bars (10) are hold by two compression spring (11) placed between the top plate (6) and the stop collars (12). By this assembly, the bottom vertical displacement of the specimen (1) at its midspan is transferred to the mounting plates (13). 
The top vertical displacements of the specimen (1) at supports are transferred to the side assemblies and from there to connecting bridges (21). Each assembly consists of a pointer (14), a locating arms (15), a guide plate (16), a guide bar (17), two sliders (18), two side screws (19), and an adjusting screw (20). The pointer (14) is located at the center of the support (2) and transfers the top vertical displacement of the specimen to the assembly through the locating arm (15). The guide plate (16) can slide freely in vertical direction along the guide bar (17) and over the back of the support block (3). Two sliders (18) are provided to reduce the friction between the guide plate (16) and the support block (3). The adjusting screw (20) is to adjust the vertical position of the side assembly.

The connecting bridges (21) are hinge connected to one of the side assemblies through the side screws (19) and sit on the two other side screws (19) of the other side assembly. The change of vertical distance between the connector bridge (21) and the mounting plate (13) located on one side of the apparatus is the net mid-span deflection of the specimen (1). This displacement can be measured by mounting a displacement transducer (LVDT) to the mounting plate (13), while the moving end of the device sits on the connecting bridge (21).

Figures H.2 through H.4 show the general view of the VSM apparatus and test setup. 

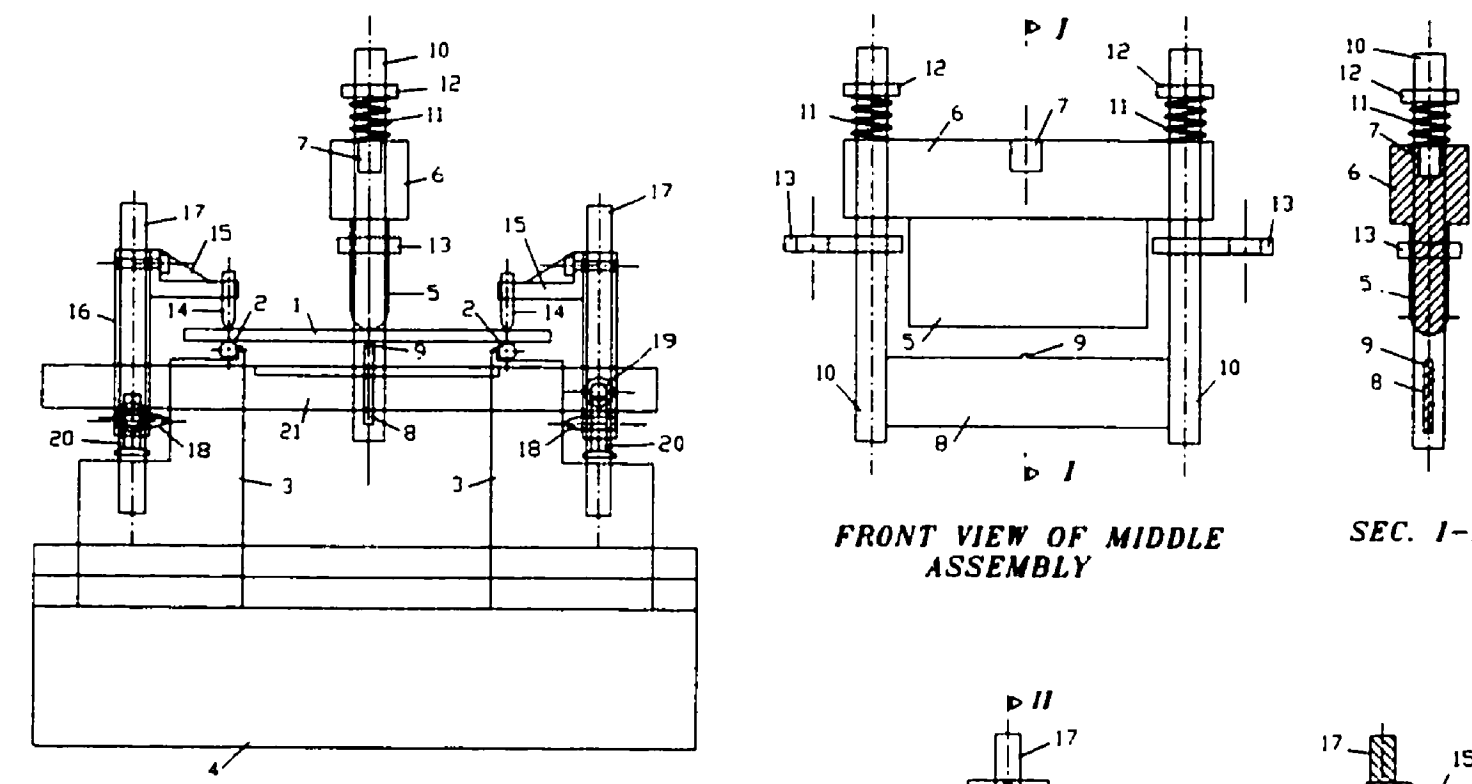

$$
\begin{array}{r}
\text { FRONT VIEW OF MIDDLE } \\
\text { ASSEMBLY }
\end{array}
$$

SEC. $1-I$

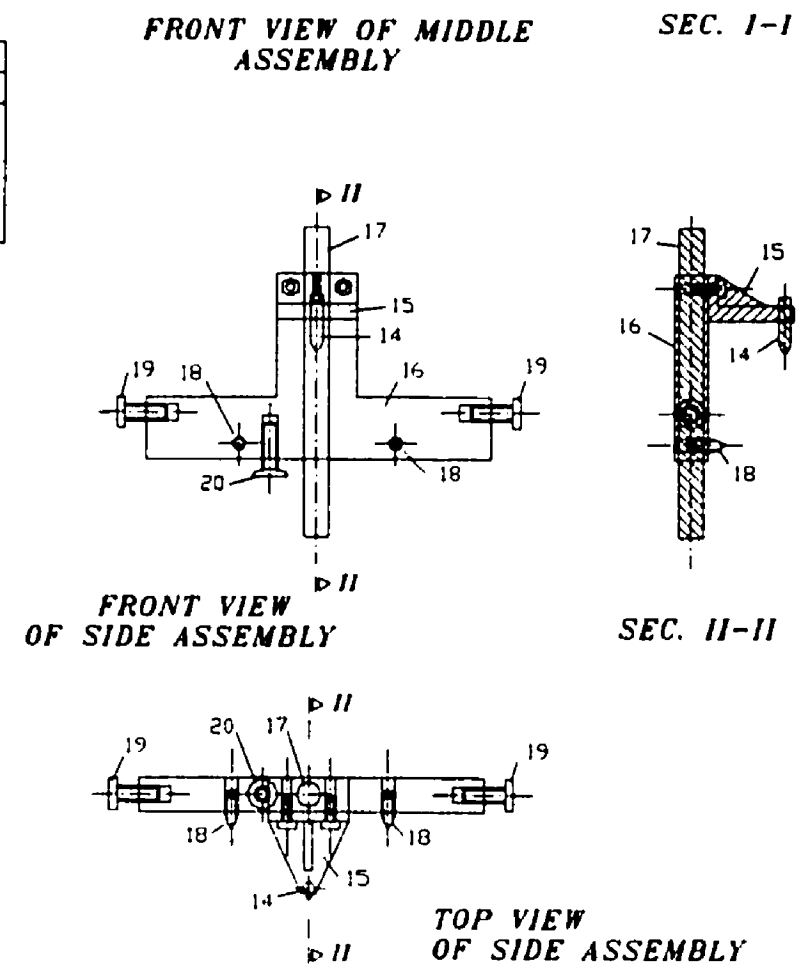

Figure H.1 The outline of the apparatus used for the VSM. 


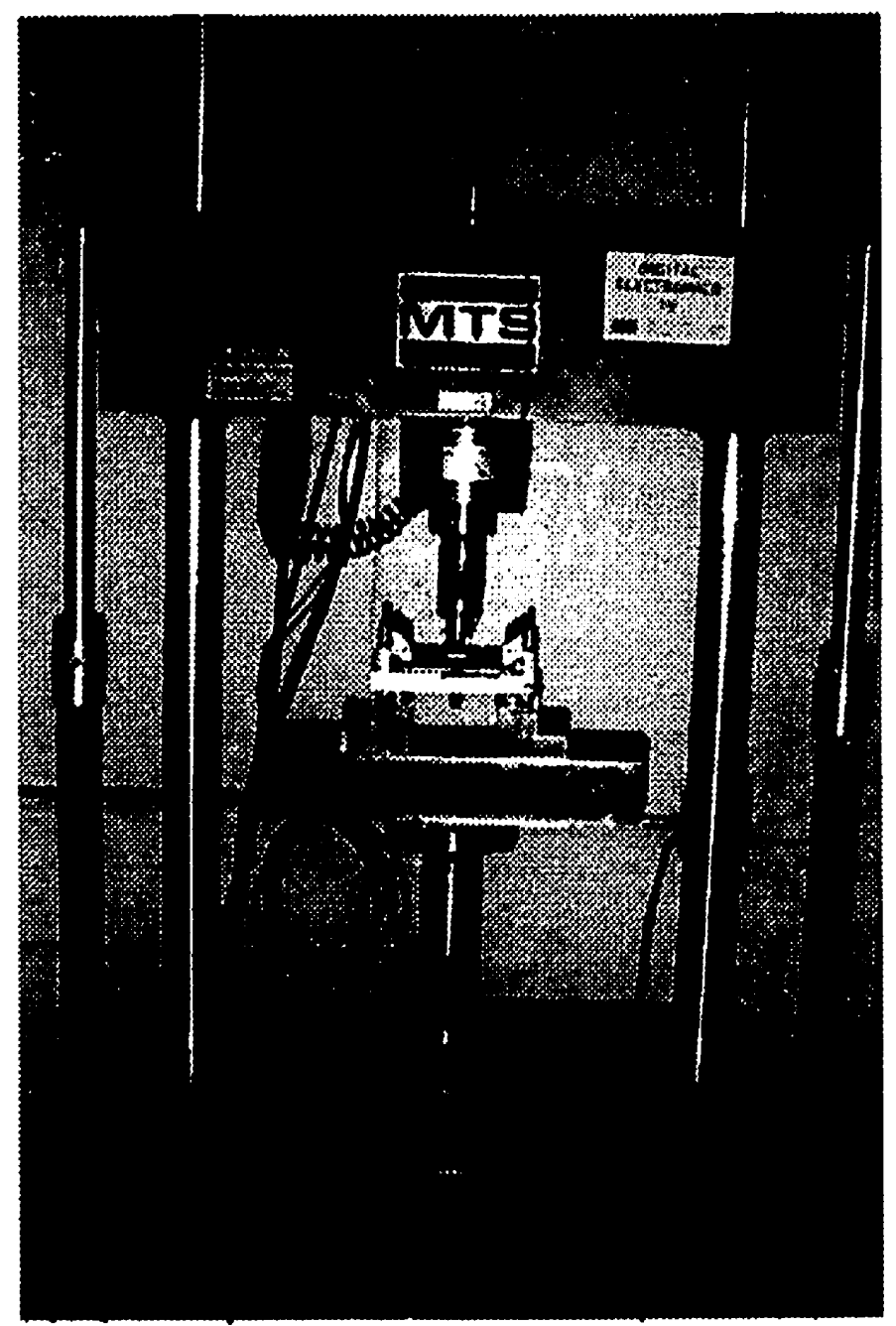

Figure H.2 The VSM apparatus installed in MTS testing machine. 


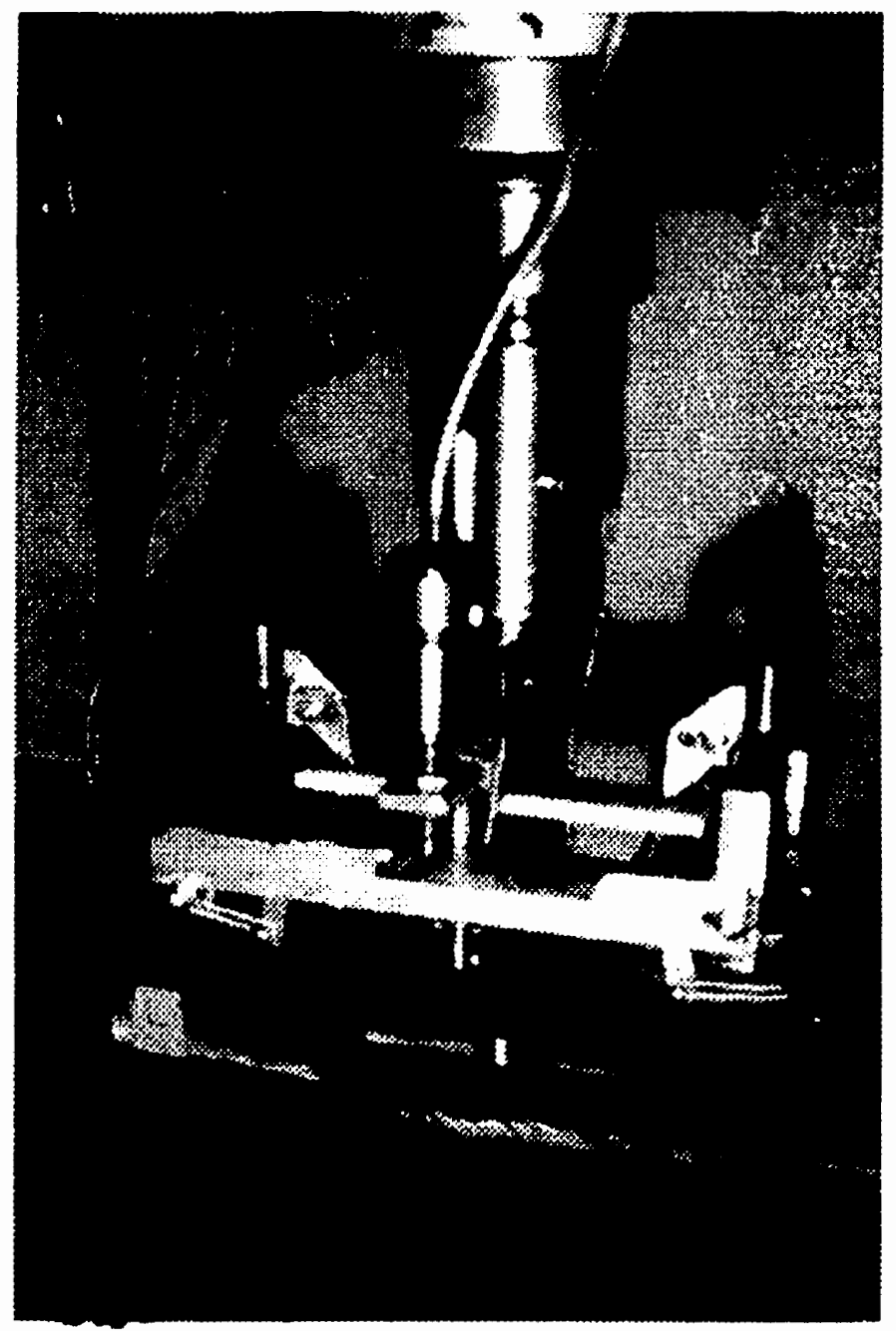

Figure H.3 E-glass/epoxy specimen in the VSM apparatus. 


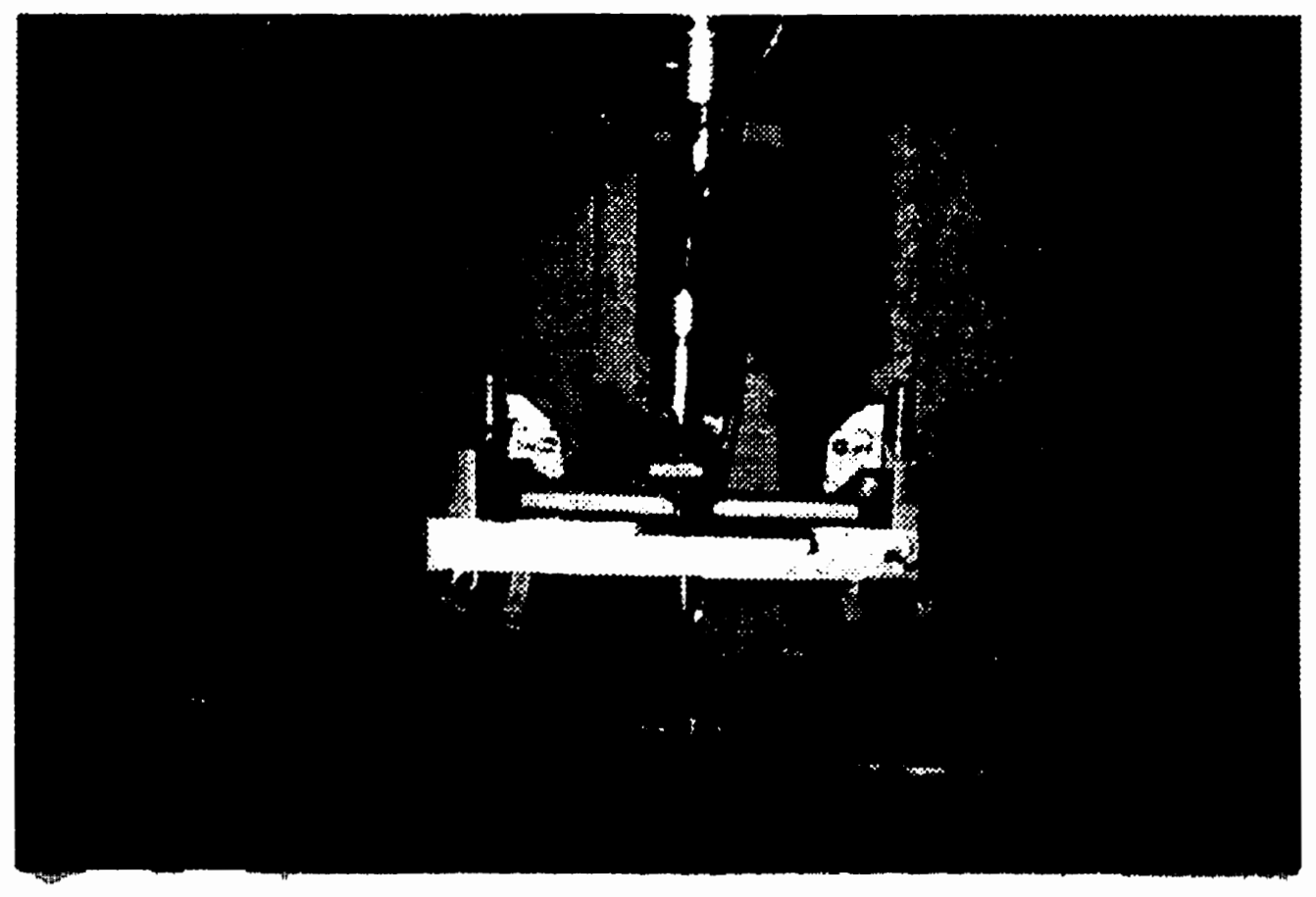

Figure H.4 Front view of the VSM apparatus. 


\section{Appendix I}

\section{SPECIFICATIONS OF THE TESTS SPECIMENS}

The specifications of the specimens used in the VSM test are tabulated in Tables I.I through I.14. The values tabulated in columns 5 of these tables are the rate of displacement of the actuator. Initial slopes of the load-deflection curve from the two consecutive tests on the specimens are presented in columns 6 and 7, respectively. The specification of the specimens used for tensile and various shear tests are tabulated in Tables I. 15 through I.21. The corresponding modulus obtained from each test is tabulated in the last column.

Table I.I Specifications of graphite/epoxy specimens in GR-1 set for the VSM.

\begin{tabular}{ccccccc}
\hline $\begin{array}{c}\text { Specimen } \\
\text { ID }\end{array}$ & $\begin{array}{c}\text { Width } \\
(\mathrm{mm})\end{array}$ & $\begin{array}{c}\text { Thickness } \\
(\mathrm{mm})\end{array}$ & $\begin{array}{c}\text { Span } \\
(\mathrm{mm})\end{array}$ & $\begin{array}{c}\text { Rate } \\
(\mathrm{mm} / \mathrm{sec})\end{array}$ & $\begin{array}{c}(\mathrm{F} / \Delta)_{1} \\
(\mathrm{~N} / \mathrm{mm})\end{array}$ & $\begin{array}{c}(\mathrm{F} / \Delta)_{2} \\
(\mathrm{~N} / \mathrm{mm})\end{array}$ \\
\hline GR-SA1 & 12.65 & 2.64 & 14.97 & 0.3 & 21417 & 21417 \\
GR-SA2 & 12.58 & 2.6 & 14.97 & 0.3 & 21160 & 21463 \\
GR-SA3 & 12.71 & 2.58 & 14.97 & 0.3 & 19563 & 21836 \\
GR-EA1 & 12.59 & 2.57 & 17.57 & 0.354 & 13350 & 13708 \\
GR-EA2 & 12.63 & 2.63 & 17.57 & 0.354 & 15336 & 15105 \\
GR-EA3 & 12.63 & 2.6 & 17.57 & 0.354 & 13742 & 14337 \\
GR-TA1 & 12.64 & 2.57 & 21.95 & 0.467 & 8599 & 8530 \\
GR-TA2 & 12.64 & 2.6 & 21.95 & 0.467 & 9136 & 9194 \\
GR-TA3 & 12.56 & 2.62 & 21.95 & 0.467 & 8885 & 8885 \\
GR-FA1 & 12.34 & 2.56 & 31.99 & 0.82 & 3126 & 3155 \\
GR-FA2 & 12.42 & 2.58 & 31.99 & 0.816 & 3256 & 3303 \\
GR-FA3 & 12.44 & 2.58 & 31.99 & 0.816 & 3221 & 3340 \\
GR-XA1 & 12.56 & 2.47 & 74.22 & 3.865 & 268 & 268.7 \\
GR-XA2 & 12.53 & 2.51 & 74.22 & 3.808 & 270.1 & 270 \\
GR-XA3 & 12.54 & 2.54 & 74.22 & 3.767 & 292.8 & 293.3 \\
\hline
\end{tabular}


Table 1.2 Specifications of graphite/epoxy specimens in GR-2 set for the VSM.

\begin{tabular}{ccccccc}
\hline $\begin{array}{c}\text { Specimen } \\
\text { ID }\end{array}$ & $\begin{array}{c}\text { Width } \\
(\mathrm{mm})\end{array}$ & $\begin{array}{c}\text { Thickness } \\
(\mathrm{mm})\end{array}$ & $\begin{array}{c}\text { Span } \\
(\mathrm{mm})\end{array}$ & $\begin{array}{c}\text { Rate } \\
(\mathrm{mm} / \mathrm{sec})\end{array}$ & $\begin{array}{c}(\mathrm{F} / \Delta)_{1} \\
(\mathrm{~N} / \mathrm{mm})\end{array}$ & $\begin{array}{c}(\mathrm{F} / \Delta)_{2} \\
(\mathrm{~N} / \mathrm{mm})\end{array}$ \\
\hline GR-SB1 & 12.41 & 2.7 & 14.97 & 0.3 & 20218 & 20122 \\
GR-SB2 & 12.52 & 2.71 & 14.97 & 0.3 & 22973 & 23031 \\
GR-SB3 & 12.58 & 2.67 & 14.97 & 0.3 & 21312 & 21507 \\
GR-EB1 & 12.41 & 2.71 & 17.57 & 0.352 & 16208 & 15576 \\
GR-EB2 & 12.6 & 2.71 & 17.57 & 0.352 & 16627 & 16570 \\
GR-EB3 & 12.53 & 2.72 & 17.57 & 0.352 & 17559 & 17333 \\
GR-TB1 & 12.59 & 2.66 & 21.95 & 0.461 & 9230 & 9303 \\
GR-TB2 & 12.52 & 2.7 & 21.95 & 0.459 & 9857 & 9767 \\
GR-TB3 & 12.4 & 2.66 & 21.95 & 0.461 & 8792 & 8835 \\
GR-FB1 & 12.45 & 2.71 & 31.99 & 0.792 & 3553 & 3657 \\
GR-FB2 & 12.44 & 2.7 & 31.99 & 0.794 & 3558 & 3735 \\
GR-FB3 & 12.44 & 2.7 & 31.99 & 0.794 & 3405 & 3675 \\
GR-XB1 & 12.66 & 2.65 & 74.22 & 3.624 & 335.5 & 335 \\
GR-XB2 & 12.66 & 2.6 & 74.22 & 3.687 & 313.3 & 320.4 \\
GR-XB3 & 12.67 & 2.65 & 74.22 & 3.624 & 335.3 & 334.8 \\
\hline & & & & & &
\end{tabular}

Table I.3 Specifications of graphite/epoxy specimens in GR-3 set for the VSM.

\begin{tabular}{ccccccc}
\hline $\begin{array}{c}\text { Specimen } \\
\text { ID }\end{array}$ & $\begin{array}{c}\text { Width } \\
(\mathrm{mm})\end{array}$ & $\begin{array}{c}\text { Thickness } \\
(\mathrm{mm})\end{array}$ & $\begin{array}{c}\text { Span } \\
(\mathrm{mm})\end{array}$ & $\begin{array}{c}\text { Rate } \\
(\mathrm{mm} / \mathrm{sec})\end{array}$ & $\begin{array}{c}(\mathrm{F} / \Delta)_{1} \\
(\mathrm{~N} / \mathrm{mm})\end{array}$ & $\begin{array}{c}(\mathrm{F} / \Delta)_{2} \\
(\mathrm{~N} / \mathrm{mm})\end{array}$ \\
\hline & width & $\mathrm{h}$ & $\mathrm{L}$ & & $\mathrm{F} / \mathrm{defl}$ & $\mathrm{F} / \mathrm{defl}$ \\
GR-SC1 & 12.59 & 2.69 & 14.97 & 0.193 & 20346 & 21422 \\
GR-SC2 & 12.51 & 2.69 & 14.97 & 0.193 & 21096 & 21648 \\
GR-SC3 & 12.52 & 2.7 & 14.97 & 0.192 & 22776 & 22776 \\
GR-EC1 & 12.49 & 2.69 & 17.57 & 0.245 & 14781 & 15317 \\
GR-EC2 & 12.6 & 2.71 & 17.57 & 0.244 & 15589 & 15764 \\
GR-EC3 & 12.62 & 2.69 & 17.57 & 0.245 & 16234 & 15530 \\
GR-TC1 & 12.66 & 2.66 & 21.95 & 0.355 & 9365 & 9229 \\
GR-TC2 & 12.63 & 2.69 & 21.95 & 0.352 & 9648 & 9712 \\
GR-TC3 & 12.65 & 2.68 & 21.95 & 0.353 & 9687 & 9613 \\
GR-FC1 & 12.36 & 2.67 & 31.99 & 0.692 & 3477 & 3587 \\
GR-FC2 & 12.4 & 2.66 & 31.99 & 0.694 & 3531 & 3550 \\
GR-FC3 & 12.36 & 2.7 & 31.99 & 0.686 & 3368 & 3628 \\
GR-XC1 & 12.55 & 2.64 & 74.22 & 3.53 & 330.1 & 329.7 \\
GR-XC2 & 12.56 & 2.64 & 74.22 & 3.53 & 336.4 & 334.6 \\
GR-XC3 & 12.56 & 2.64 & 74.22 & 3.53 & 336.8 & 335.2 \\
\hline
\end{tabular}


Table I.4 Specifications of graphite/epoxy specimens in GR-4 set for the VSM.

\begin{tabular}{ccccccc}
\hline $\begin{array}{c}\text { Specimen } \\
\text { ID }\end{array}$ & $\begin{array}{c}\text { Width } \\
(\mathrm{mm})\end{array}$ & $\begin{array}{c}\text { Thickness } \\
(\mathrm{mm})\end{array}$ & $\begin{array}{c}\text { Span } \\
(\mathrm{mm})\end{array}$ & $\begin{array}{c}\text { Rate } \\
(\mathrm{mm} / \mathrm{sec})\end{array}$ & $\begin{array}{c}(\mathrm{F} / \Delta)_{1} \\
(\mathrm{~N} / \mathrm{mm})\end{array}$ & $\begin{array}{c}(\mathrm{F} / \Delta)_{2} \\
(\mathrm{~N} / \mathrm{mm})\end{array}$ \\
\hline GR-SD1 & 12.48 & 2.59 & 14.97 & 0.173 & 21429 & 21429 \\
GR-SD2 & 12.5 & 2.59 & 14.97 & 0.173 & 22696 & 22578 \\
GR-SD3 & 12.54 & 2.58 & 14.97 & 0.174 & 21062 & 20680 \\
GR-ED1 & 12.49 & 2.61 & 17.57 & 0.238 & 15042 & 14872 \\
GR-ED2 & 12.47 & 2.6 & 17.57 & 0.239 & 14697 & 14654 \\
GR-ED3 & 12.47 & 2.61 & 17.57 & 0.238 & 14690 & 13946 \\
GR-TD1 & 12.52 & 2.59 & 21.95 & 0.394 & 8870 & 8790 \\
GR-TD2 & 12.5 & 2.61 & 21.95 & 0.39 & 8419 & 8646 \\
GR-TD3 & 12.48 & 2.57 & 21.95 & 0.399 & 8424 & 8571 \\
GR-FD1 & 12.45 & 2.57 & 31.99 & 1.018 & 3052 & 3062 \\
GR-FD2 & 12.44 & 2.57 & 31.99 & 1.018 & 3111 & 3202 \\
GR-FD3 & 12.5 & 2.59 & 31.99 & 1.005 & 3381 & 3358 \\
GR-XD1 & 12.44 & 2.54 & 74.22 & 11.007 & 291.1 & 290.3 \\
GR-XD2 & 12.52 & 2.51 & 74.22 & 11.261 & 286.2 & 284.2 \\
GR-XD3 & 12.48 & 2.53 & 74.22 & 11.091 & 295.5 & 294.9 \\
\hline
\end{tabular}

Table I.5 Specifications of Kevlar/epoxy specimens in K-1 set for the VSM.

\begin{tabular}{ccccccc}
\hline $\begin{array}{c}\text { Specimen } \\
\text { ID }\end{array}$ & $\begin{array}{c}\text { Width } \\
(\mathrm{mm})\end{array}$ & $\begin{array}{c}\text { Thickness } \\
(\mathrm{mm})\end{array}$ & $\begin{array}{c}\text { Span } \\
(\mathrm{mm})\end{array}$ & $\begin{array}{c}\text { Rate } \\
(\mathrm{mm} / \mathrm{sec})\end{array}$ & $\begin{array}{c}(\mathrm{F} / \Delta)_{1} \\
(\mathrm{~N} / \mathrm{mm})\end{array}$ & $\begin{array}{c}(\mathrm{F} / \Delta)_{2} \\
(\mathrm{~N} / \mathrm{mm})\end{array}$ \\
\hline K-S05 & 12.56 & 1.86 & 13.55 & 0.276 & 5106.8 & 5106.8 \\
K-S06 & 12.52 & 1.91 & 13.55 & 0.275 & 5661.6 & 5409 \\
K-S07 & 11.24 & 1.93 & 13.55 & 0.274 & 4858.1 & 4594 \\
K-E01 & 12.58 & 1.86 & 15.13 & 0.317 & 3950.1 & 3933.9 \\
K-E02 & 12.57 & 1.91 & 15.13 & 0.314 & 4305 & 4280.4 \\
K-E03 & 12.59 & 1.88 & 15.13 & 0.316 & 4220.8 & 4203.2 \\
K-E04 & 12.67 & 1.9 & 15.13 & 0.315 & 4091.7 & 4133.9 \\
K-T01 & 12.3 & 1.92 & 18.97 & 0.428 & 2183.4 & 2324 \\
K-T02 & 12.44 & 1.92 & 18.97 & 0.428 & 2594.9 & 2477.5 \\
K-T03 & 12.54 & 1.91 & 18.97 & 0.429 & 2401.2 & 2352.4 \\
K-T04 & 12.34 & 1.86 & 18.97 & 0.434 & 2114.04 & 2072 \\
K-F01 & 12.5 & 1.93 & 26.51 & 0.723 & 1066.6 & 1063 \\
K-F02 & 12.56 & 1.9 & 26.51 & 0.73 & 1073 & 1076 \\
K-F03 & 12.05 & 1.86 & 26.51 & 0.741 & 922.8 & 899 \\
K-X01 & 12.63 & 1.91 & 56.92 & 2.942 & 118.9 & 116.5 \\
K-X02 & 12.63 & 1.91 & 56.92 & 2.942 & 125.1 & 123.3 \\
K-X03 & 12.51 & 1.93 & 56.92 & 2.914 & 125.1 & 122.9 \\
\hline
\end{tabular}


Table I.6 Specifications of Kevlar/epoxy specimens in K-2 set for the VSM.

\begin{tabular}{ccccccc}
$\begin{array}{c}\text { Specimen } \\
\text { ID }\end{array}$ & $\begin{array}{c}\text { Width } \\
(\mathrm{mm})\end{array}$ & $\begin{array}{c}\text { Thickness } \\
(\mathrm{mm})\end{array}$ & $\begin{array}{c}\text { Span } \\
(\mathrm{mm})\end{array}$ & $\begin{array}{c}\text { Rate } \\
(\mathrm{mm} / \mathrm{sec})\end{array}$ & $\begin{array}{c}(\mathrm{F} / \Delta)_{1} \\
(\mathrm{~N} / \mathrm{mm})\end{array}$ & $\begin{array}{c}(\mathrm{F} / \Delta)_{2} \\
(\mathrm{~N} / \mathrm{mm})\end{array}$ \\
\hline K-S08 & 12.59 & 1.91 & 13.55 & 0.275 & 5369.3 & 5102 \\
K-S09 & 12.49 & 1.91 & 13.55 & 0.275 & 5241.6 & 5060 \\
K-S10 & 12.21 & 1.93 & 13.55 & 0.274 & 5468.8 & 5118 \\
K-E05 & 12.6 & 1.87 & 15.13 & 0.316 & 4288.9 & 4282.5 \\
K-E06 & 12.47 & 1.86 & 15.13 & 0.317 & 3852.71 & 3952 \\
K-E07 & 12.46 & 1.92 & 15.13 & 0.314 & 4232.11 & 4192 \\
K-E08 & 12.58 & 1.93 & 15.13 & 0.313 & 4347.2 & 4243 \\
K-T05 & 12.57 & 1.9 & 18.97 & 0.43 & 2414.8 & 2351 \\
K-T06 & 12.43 & 1.86 & 18.97 & 0.434 & 2153.8 & 2093 \\
K-T07 & 12.54 & 1.87 & 18.97 & 0.433 & 2426.6 & 2376 \\
K-F04 & 12.41 & 1.91 & 26.51 & 0.728 & 1015.5 & 1000 \\
K-F05 & 12.43 & 1.93 & 26.51 & 0.723 & 1071 & 1053 \\
K-F06 & 12.56 & 1.87 & 26.51 & 0.739 & 1014.5 & 1018 \\
K-F07 & 12.46 & 1.93 & 26.51 & 0.723 & 1061.4 & 1055 \\
K-X04 & 12.49 & 1.93 & 56.92 & 2.914 & 127.1 & 127.1 \\
K-X05 & 12.46 & 1.86 & 56.92 & 3.015 & 118.8 & 119.4 \\
K-X06 & 12.38 & 1.93 & 56.92 & 2.914 & 112.1 & 122.5 \\
K-X07 & 12.58 & 1.92 & 56.92 & 2.928 & 118.9 & 119.4 \\
\hline
\end{tabular}

Table I.7 Specifications of Kevlar/epoxy specimens in K-3 set for the VSM.

\begin{tabular}{ccccccc}
\hline $\begin{array}{c}\text { Specimen } \\
\text { ID }\end{array}$ & $\begin{array}{c}\text { Width } \\
(\mathrm{mm})\end{array}$ & $\begin{array}{c}\text { Thickness } \\
(\mathrm{mm})\end{array}$ & $\begin{array}{c}\text { Span } \\
(\mathrm{mm})\end{array}$ & $\begin{array}{c}\text { Rate } \\
(\mathrm{mm} / \mathrm{sec})\end{array}$ & $\begin{array}{c}(\mathrm{F} / \Delta)_{1} \\
(\mathrm{~N} / \mathrm{mm})\end{array}$ & $\begin{array}{c}(\mathrm{F} / \Delta)_{2} \\
(\mathrm{~N} / \mathrm{mm})\end{array}$ \\
\hline K-S11 & 12.39 & 1.88 & 13.55 & 0.2 & 4910 & 4992 \\
K-S12 & 12.54 & 1.93 & 13.55 & 0.197 & 5266.7 & 5292 \\
K-S13 & 12.53 & 1.92 & 13.55 & 0.198 & 5008.6 & 4773 \\
K-E09 & 11.79 & 1.92 & 15.13 & 0.237 & 3885.2 & 3798 \\
K-E10 & 12.52 & 1.89 & 15.13 & 0.24 & 4052.3 & 4003 \\
K-E11 & 12.63 & 1.91 & 15.13 & 0.238 & 4140.3 & 4101 \\
K-T08 & 12.53 & 1.94 & 18.97 & 0.348 & 2482.1 & 2362 \\
K-T09 & 12.56 & 1.87 & 18.97 & 0.358 & 2503.4 & 2428 \\
K-T10 & 12.56 & 1.91 & 18.97 & 0.352 & 2504.1 & 2461 \\
K-F08 & 12.5 & 1.9 & 26.51 & 0.654 & 1062.8 & 1055 \\
K-F09 & 12.55 & 1.86 & 26.51 & 0.667 & 954.3 & 939 \\
K-F10 & 12.53 & 1.86 & 26.51 & 0.667 & 954 & 944 \\
K-X08 & 12.59 & 1.86 & 56.92 & 2.94 & 110.1 & 110.2 \\
K-X09 & 12.5 & 1.9 & 56.92 & 2.88 & 124.2 & 125 \\
K-X10 & 12.44 & 1.89 & 56.92 & 2.895 & 120.5 & 122.1 \\
\hline
\end{tabular}


Table I.8 Specifications of Kevlar/epoxy specimens in K-4 set for the VSM.

\begin{tabular}{ccccccc}
\hline $\begin{array}{c}\text { Specimen } \\
\text { ID }\end{array}$ & $\begin{array}{c}\text { Width } \\
(\mathrm{mm})\end{array}$ & $\begin{array}{c}\text { Thickness } \\
(\mathrm{mm})\end{array}$ & $\begin{array}{c}\text { Span } \\
(\mathrm{mm})\end{array}$ & $\begin{array}{c}\text { Rate } \\
(\mathrm{mm} / \mathrm{sec})\end{array}$ & $\begin{array}{c}(\mathrm{F} / \Delta)_{1} \\
(\mathrm{~N} / \mathrm{mm})\end{array}$ & $\begin{array}{c}(\mathrm{F} / \Delta)_{2} \\
(\mathrm{~N} / \mathrm{mm})\end{array}$ \\
\hline K-S14 & 12.59 & 1.9 & 13.55 & 0.199 & 5192.2 & 5236 \\
K-S15 & 12.5 & 1.92 & 13.55 & 0.198 & 5210.6 & 5268 \\
K-S16 & 12.54 & 1.86 & 13.55 & 0.202 & 4884 & 4839 \\
K-E12 & 12.55 & 1.91 & 15.13 & 0.238 & 4420.3 & 4357 \\
K-E13 & 12.59 & 1.89 & 15.13 & 0.24 & 4225.4 & 4117 \\
K-E14 & 12.52 & 1.91 & 15.13 & 0.238 & 4192.5 & 4083 \\
K-E15 & 12.55 & 1.9 & 15.13 & 0.239 & 4282.6 & 4210 \\
K-T11 & 12.54 & 1.86 & 18.97 & 0.36 & 2342.4 & 2303 \\
K-T12 & 12.48 & 1.91 & 18.97 & 0.352 & 2389.4 & 2345 \\
K-T13 & 12.62 & 1.91 & 18.97 & 0.352 & 2522.8 & 2465 \\
K-F11 & 12.36 & 1.87 & 26.51 & 0.664 & 924.3 & 909 \\
K-F12 & 12.58 & 1.87 & 26.51 & 0.664 & 1056.3 & 1035 \\
K-F13 & 12.59 & 1.92 & 26.51 & 0.648 & 1049.9 & 1046 \\
K-X11 & 12.39 & 1.95 & 56.92 & 2.808 & 121.5 & 121.8 \\
K-X12 & 12.57 & 1.86 & 56.92 & 2.94 & 120.7 & 121.1 \\
K-X13 & 12.58 & 1.91 & 56.92 & 2.865 & 119.9 & 120.3 \\
\hline
\end{tabular}

Table I.9 Specifications of Kevlar/epoxy specimens in K-5 set for the VSM.

\begin{tabular}{ccccccc}
\hline $\begin{array}{c}\text { Specimen } \\
\text { ID }\end{array}$ & $\begin{array}{c}\text { Width } \\
(\mathrm{mm})\end{array}$ & $\begin{array}{c}\text { Thickness } \\
(\mathrm{mm})\end{array}$ & $\begin{array}{c}\text { Span } \\
(\mathrm{mm})\end{array}$ & $\begin{array}{c}\text { Rate } \\
(\mathrm{mm} / \mathrm{sec})\end{array}$ & $\begin{array}{c}(\mathrm{F} / \Delta)_{1} \\
(\mathrm{~N} / \mathrm{mm})\end{array}$ & $\begin{array}{c}(\mathrm{F} / \Delta)_{2} \\
(\mathrm{~N} / \mathrm{mm})\end{array}$ \\
\hline K-S17 & 12.52 & 1.86 & 13.55 & 0.201 & 4561.3 & 4480 \\
K-S18 & 12.46 & 1.89 & 13.55 & 0.197 & 5381.9 & 5407 \\
K-S19 & 12.53 & 1.92 & 13.55 & 0.194 & 5357.4 & 5270 \\
K-E16 & 12.54 & 1.91 & 15.13 & 0.249 & 4215.3 & 4210 \\
K-E17 & 12.52 & 1.9 & 15.13 & 0.251 & 4259.3 & 4185 \\
K-E18 & 12.49 & 1.9 & 15.13 & 0.251 & 4106.1 & 4019 \\
K-E19 & 12.55 & 1.84 & 15.13 & 0.261 & 3731 & 3634 \\
K-T14 & 12.62 & 1.92 & 18.97 & 0.422 & 2473.3 & 2456 \\
K-T15 & 12.56 & 1.87 & 18.97 & 0.439 & 2202.2 & 2147 \\
K-T16 & 12.69 & 1.91 & 18.97 & 0.426 & 2372.4 & 2321 \\
K-F14 & 12.62 & 1.86 & 26.51 & 1.057 & 1038.7 & 1039 \\
K-F15 & 12.61 & 1.88 & 26.51 & 1.038 & 951 & 943 \\
K-F16 & 12.56 & 1.9 & 26.51 & 1.019 & 1042.7 & 1040 \\
K-X14 & 12.62 & 1.93 & 56.92 & 8.593 & 128.7 & 128.4 \\
K-X15 & 12.58 & 1.9 & 56.92 & 8.856 & 127.3 & 126.9 \\
K-X16 & 12.58 & 1.93 & 56.92 & 8.593 & 129.3 & 129 \\
K-X17 & 12.59 & 1.91 & 56.92 & 8.767 & 117.6 & 117.7 \\
\hline
\end{tabular}


Table I. 10 Specifications of Kevlar/epoxy specimens in K-6 set for the VSM.

\begin{tabular}{ccccccc}
\hline $\begin{array}{c}\text { Specimen } \\
\text { ID }\end{array}$ & $\begin{array}{c}\text { Width } \\
(\mathrm{mm})\end{array}$ & $\begin{array}{c}\text { Thickness } \\
(\mathrm{mm})\end{array}$ & $\begin{array}{c}\text { Span } \\
(\mathrm{mm})\end{array}$ & $\begin{array}{c}\text { Rate } \\
(\mathrm{mm} / \mathrm{sec})\end{array}$ & $\begin{array}{c}(\mathrm{F} / \Delta)_{1} \\
(\mathrm{~N} / \mathrm{mm})\end{array}$ & $\begin{array}{c}(\mathrm{F} / \Delta)_{2} \\
(\mathrm{~N} / \mathrm{mm})\end{array}$ \\
\hline K-S20 & 12.51 & 1.86 & 13.55 & 0.201 & 5053.5 & 4872 \\
K-S21 & 12.38 & 1.89 & 13.55 & 0.197 & 4959.3 & 5262 \\
K-S22 & 12.44 & 1.92 & 13.55 & 0.194 & 5340.9 & 5301 \\
K-E20 & 12.55 & 1.84 & 15.13 & 0.261 & 4095 & 4047 \\
K-E21 & 12.57 & 1.84 & 15.13 & 0.261 & 3957.1 & 3985 \\
K-E22 & 12.46 & 1.88 & 15.13 & 0.254 & 3884 & 3851 \\
K-T17 & 12.55 & 1.89 & 18.97 & 0.432 & 2436.7 & 2369 \\
K-T18 & 12.51 & 1.93 & 18.97 & 0.419 & 2571.9 & 2491 \\
K-T19 & 12.63 & 1.86 & 18.97 & 0.443 & 2417.1 & 2385 \\
K-F17 & 12.68 & 1.93 & 26.51 & 0.993 & 1105.9 & 1087 \\
K-F18 & 12.54 & 1.95 & 26.51 & 0.976 & 1123.8 & 1105 \\
K-F19 & 12.66 & 1.91 & 26.51 & 1.01 & 1030.3 & 1019 \\
K-F20 & 12.51 & 1.92 & 26.51 & 1.001 & 1042.7 & 1044 \\
K-X18 & 12.53 & 1.93 & 56.92 & 8.593 & 131.3 & 131.1 \\
K-X19 & 12.57 & 1.9 & 56.92 & 8.856 & 124.4 & 124.5 \\
K-X20 & 12.54 & 1.85 & 56.92 & 9.322 & 108.2 & 108.7 \\
\hline
\end{tabular}

Table I.11 Specifications of E-glass/epoxy specimens in GL-1 set for the VSM.

\begin{tabular}{ccccccc}
\hline $\begin{array}{c}\text { Specimen } \\
\text { ID }\end{array}$ & $\begin{array}{c}\text { Width } \\
(\mathrm{mm})\end{array}$ & $\begin{array}{c}\text { Thickness } \\
(\mathrm{mm})\end{array}$ & $\begin{array}{c}\text { Span } \\
(\mathrm{mm})\end{array}$ & $\begin{array}{c}\text { Rate } \\
(\mathrm{mm} / \mathrm{sec})\end{array}$ & $\begin{array}{c}(\mathrm{F} / \Delta)_{1} \\
(\mathrm{~N} / \mathrm{mm})\end{array}$ & $\begin{array}{c}(\mathrm{F} / \Delta)_{2} \\
(\mathrm{~N} / \mathrm{mm})\end{array}$ \\
\hline GL-S01 & 13.28 & 6.32 & 31.19 & 0.636 & 18806 & 18529 \\
GL-S02 & 13.31 & 6.33 & 31.19 & 0.636 & 18660 & 18513 \\
GL-S03 & 13.5 & 6.33 & 31.19 & 0.636 & 17863 & 18151 \\
GL-E01 & 13.55 & 6.3 & 37.53 & 0.751 & 11265 & 10896 \\
GL-E02 & 13.27 & 6.32 & 37.53 & 0.751 & 10445 & 10679 \\
GL-E03 & 13.44 & 6.33 & 37.53 & 0.751 & 11526 & 11252 \\
GL-T01 & 13.33 & 6.32 & 51.18 & 1.07 & 4395 & 4508 \\
GL-T02 & 13.25 & 6.33 & 51.18 & 1.07 & 4698 & 4660 \\
GL-T03 & 13.53 & 6.3 & 51.18 & 1.07 & 4484 & 4389 \\
GL-F01 & 13.41 & 6.32 & 112.24 & 3.701 & 453.3 & 452.8 \\
GL-F02 & 13.44 & 6.31 & 112.24 & 3.706 & 439.3 & 437.9 \\
GL-F03 & 13.45 & 6.31 & 112.24 & 3.706 & 456.8 & 453.2 \\
\hline
\end{tabular}


Table I.12 Specifications of E-glass /epoxy specimens in GL-2 set for the VSM.

\begin{tabular}{ccccccc}
\hline $\begin{array}{c}\text { Specimen } \\
\text { ID }\end{array}$ & $\begin{array}{c}\text { Width } \\
(\mathrm{mm})\end{array}$ & $\begin{array}{c}\text { Thickness } \\
(\mathrm{mm})\end{array}$ & $\begin{array}{c}\text { Span } \\
(\mathrm{mm})\end{array}$ & $\begin{array}{c}\text { Rate } \\
(\mathrm{mm} / \mathrm{sec})\end{array}$ & $\begin{array}{c}(\mathrm{F} / \Delta)_{1} \\
(\mathrm{~N} / \mathrm{mm})\end{array}$ & $\begin{array}{c}(\mathrm{F} / \Delta)_{2} \\
(\mathrm{~N} / \mathrm{mm})\end{array}$ \\
\hline GL-S04 & 13.3 & 6.3 & 31.19 & 0.383 & 16802 & 17883 \\
GL-S05 & 13.48 & 6.33 & 31.19 & 0.383 & 18631 & 18631 \\
GL-S06 & 13.3 & 6.3 & 31.19 & 0.383 & 16905 & 16770 \\
GL-E04 & 13.62 & 6.34 & 37.53 & 0.497 & 11266 & 11768 \\
GL-E05 & 13.27 & 6.33 & 37.53 & 0.497 & 11172 & 11282 \\
GL-E06 & 13.43 & 6.32 & 37.53 & 0.498 & 10240 & 10597 \\
GL-T04 & 13.26 & 6.33 & 51.15 & 0.818 & 4747 & 4665 \\
GL-T05 & 13.34 & 6.33 & 51.15 & 0.818 & 4840 & 4746 \\
GL-T06 & 13.51 & 6.34 & 51.15 & 0.818 & 4833 & 4883 \\
GL-F04 & 13.25 & 6.33 & 112.24 & 3.444 & 467 & 463.2 \\
GL-F05 & 13.44 & 6.33 & 112.24 & 3.444 & 469.9 & 470.2 \\
GL-F06 & 13.44 & 6.33 & 112.24 & 3.444 & 470.2 & 470.4 \\
\hline
\end{tabular}

Table I.13 Specifications of E-glass/epoxy specimens in GL-3 set for the VSM.

\begin{tabular}{ccccccc}
\hline $\begin{array}{c}\text { Specimen } \\
\text { ID }\end{array}$ & $\begin{array}{c}\text { Width } \\
(\mathrm{mm})\end{array}$ & $\begin{array}{c}\text { Thickness } \\
(\mathrm{mm})\end{array}$ & $\begin{array}{c}\text { Span } \\
(\mathrm{mm})\end{array}$ & $\begin{array}{c}\text { Rate } \\
(\mathrm{mm} / \mathrm{sec})\end{array}$ & $\begin{array}{c}(\mathrm{F} / \Delta)_{1} \\
(\mathrm{~N} / \mathrm{mm})\end{array}$ & $\begin{array}{c}(\mathrm{F} / \Delta)_{2} \\
(\mathrm{~N} / \mathrm{mm})\end{array}$ \\
\hline GL-S07 & 13.48 & 6.32 & 31.19 & 0.383 & 19415 & 19817 \\
GL-S08 & 12.7 & 6.29 & 31.19 & 0.383 & 14976 & 15375 \\
GL-S09 & 13.57 & 6.32 & 31.19 & 0.383 & 17845 & 18215 \\
GL-E07 & 13.65 & 6.3 & 37.53 & 0.499 & 10540 & 10690 \\
GL-E08 & 13.33 & 6.32 & 37.53 & 0.498 & 10760 & 10727 \\
GL-E09 & 13.53 & 6.3 & 37.53 & 0.499 & 10779 & 10792 \\
GL-T07 & 13.39 & 6.3 & 51.15 & 0.818 & 4283 & 4275 \\
GL-T08 & 13.57 & 6.3 & 51.15 & 0.818 & 4401 & 4410 \\
GL-T09 & 13.21 & 6.3 & 51.15 & 0.818 & 4393 & 4282 \\
GL-F07 & 13.52 & 6.3 & 112.24 & 3.459 & 440.5 & 439.4 \\
GL-F08 & 13.45 & 6.3 & 112.24 & 3.459 & 444 & 437.1 \\
GL-F09 & 13.45 & 6.31 & 112.24 & 3.454 & 457.3 & 448.9 \\
\hline
\end{tabular}




\begin{tabular}{ccccccc}
\multicolumn{6}{c}{ Table I.14 Specifications of E-glass/epoxy specimens in GL-4 set for the VSM. } \\
\hline $\begin{array}{c}\text { Specimen } \\
\text { ID }\end{array}$ & $\begin{array}{c}\text { Width } \\
(\mathrm{mm})\end{array}$ & $\begin{array}{c}\text { Thickness } \\
(\mathrm{mm})\end{array}$ & $\begin{array}{c}\text { Span } \\
(\mathrm{mm})\end{array}$ & $\begin{array}{c}\text { Rate } \\
(\mathrm{mm} / \mathrm{sec})\end{array}$ & $\begin{array}{c}(\mathrm{F} / \Delta)_{1} \\
(\mathrm{~N} / \mathrm{mm})\end{array}$ & $\begin{array}{c}(\mathrm{F} / \Delta)_{2} \\
(\mathrm{~N} / \mathrm{mm})\end{array}$ \\
\hline GL-S10 & 13.62 & 6.31 & 31.19 & 0.568 & 17838 & 17697 \\
GL-S11 & 12.41 & 6.29 & 31.19 & 0.571 & 15559 & 15641 \\
GL-S12 & 13.58 & 6.32 & 31.19 & 0.567 & 15763 & 17142 \\
GL-E10 & 13.29 & 6.3 & 37.53 & 0.891 & 10364 & 10741 \\
GL-E11 & 13.35 & 6.33 & 37.53 & 0.885 & 11983 & 11914 \\
GL-E12 & 13.48 & 6.33 & 37.53 & 0.885 & 10921 & 10891 \\
GL-T10 & 13.24 & 6.32 & 51.18 & 1.99 & 4576 & 4392 \\
GL-T11 & 13.55 & 6.33 & 51.18 & 1.99 & 4622 & 4524 \\
GL-T12 & 13.31 & 6.32 & 51.18 & 1.99 & 4540 & 4419 \\
GL-F10 & 13.49 & 6.33 & 112.24 & 18.32 & 472.3 & 473.1 \\
GL-F11 & 13.5 & 6.32 & 112.24 & 18.37 & 454.4 & 451.4 \\
GL-F12 & 13.42 & 6.3 & 112.24 & 18.49 & 438.8 & 438.2 \\
\hline
\end{tabular}

Table I.15 Specifications of graphite/epoxy specimens in tensile test.

\begin{tabular}{ccccc}
$\begin{array}{c}\text { Specimen } \\
\text { ID }\end{array}$ & $\begin{array}{c}\text { Width } \\
(\mathrm{mm})\end{array}$ & $\begin{array}{c}\text { Thickness } \\
(\mathrm{mm})\end{array}$ & $\begin{array}{c}\text { Length } \\
(\mathrm{mm})\end{array}$ & $\begin{array}{c}E \\
(\mathrm{GPa})\end{array}$ \\
\hline GR-TN-1 & 12.15 & 2.33 & 140 & 149.8 \\
GR-TN-2 & 12.15 & 2.36 & 140 & 147.2 \\
GR-TN-3 & 12.21 & 2.49 & 140 & 153.3 \\
GR-TN-4 & 13.81 & 2.62 & 290 & 150.1 \\
\hline
\end{tabular}

Table I.16 Specifications of E-glass/epoxy specimens in tensile test.

\begin{tabular}{ccccc}
\hline $\begin{array}{c}\text { Specimen } \\
\text { ID }\end{array}$ & $\begin{array}{c}\text { Width } \\
(\mathrm{mm})\end{array}$ & $\begin{array}{c}\text { Thickness } \\
(\mathrm{mm})\end{array}$ & $\begin{array}{c}\text { Length } \\
(\mathrm{mm})\end{array}$ & $\begin{array}{c}E \\
(\mathrm{GPa})\end{array}$ \\
\hline GL-TN-1 & 14.28 & 6.26 & 200 & 47.2 \\
GL-TN-2 & 13.46 & 6.25 & 200 & 50.6 \\
GL-TN-3 & 14.27 & 6.27 & 200 & 47.7 \\
\hline
\end{tabular}


Table 1.17 Specifications of graphite/epoxy specimens in losipescu shear test.

\begin{tabular}{ccccc}
$\begin{array}{c}\text { Specimen } \\
\text { ID }\end{array}$ & $\begin{array}{c}\text { Width } \\
(\mathrm{mm})\end{array}$ & $\begin{array}{c}\text { Thickness } \\
(\mathrm{mm})\end{array}$ & $\begin{array}{c}\text { Length } \\
(\mathrm{mm})\end{array}$ & $\begin{array}{c}G \\
(\mathrm{GPa})\end{array}$ \\
\hline GR-ISH-1 & 11.37 & 2.56 & 760 & $\mathbf{5 . 5 3}$ \\
GR-ISH-2 & 11.34 & 2.55 & 760 & 6.11 \\
GR-ISH-3 & 11.37 & 2.58 & 760 & 6.51 \\
\hline
\end{tabular}

Table I.18 Specifications of Kevlar/epoxy specimens in losipescu shear test

\begin{tabular}{ccccc}
\hline $\begin{array}{c}\text { Specimen } \\
\text { ID }\end{array}$ & $\begin{array}{c}\text { Width } \\
(\mathrm{mm})\end{array}$ & $\begin{array}{c}\text { Thickness } \\
(\mathrm{mm})\end{array}$ & $\begin{array}{c}\text { Length } \\
(\mathrm{mm})\end{array}$ & $\begin{array}{c}(; \\
(\mathrm{GPa})\end{array}$ \\
\hline K-ISH-1 & 10.97 & 1.83 & 760 & 2.09 \\
K-ISH-2 & 11.21 & 1.83 & 760 & 2.13 \\
K-ISH-3 & 10.80 & 1.91 & 760 & 2.24 \\
\hline
\end{tabular}

Table I.19 Specifications of E-glass/epoxy specimens in Iosipescu shear test.

\begin{tabular}{ccccc}
\hline $\begin{array}{c}\text { Specimen } \\
\text { ID }\end{array}$ & $\begin{array}{c}\text { Width } \\
(\mathrm{mm})\end{array}$ & $\begin{array}{c}\text { Thickness } \\
(\mathrm{mm})\end{array}$ & $\begin{array}{c}\text { Length } \\
(\mathrm{mm})\end{array}$ & $\begin{array}{c}\dot{(G)} \\
(\mathrm{GPa})\end{array}$ \\
\hline GL-ISH-1 & 10.57 & 6.28 & 760 & 7.07 \\
GL-ISH-2 & 10.64 & 6.28 & 760 & 7.39 \\
GL-ISH-3 & 10.52 & 6.29 & 760 & $\mathbf{8 . 2 7}$ \\
\hline
\end{tabular}

Table 1.20 Specifications of graphite/epoxy specimens in $\pm 45^{\circ}$ shear test.

\begin{tabular}{ccccc}
\hline $\begin{array}{c}\text { Specimen } \\
\text { ID }\end{array}$ & $\begin{array}{c}\text { Width } \\
(\mathrm{mm})\end{array}$ & $\begin{array}{c}\text { Thickness } \\
(\mathrm{mm})\end{array}$ & $\begin{array}{c}\text { Length } \\
(\mathrm{mm})\end{array}$ & $\begin{array}{c}(i \\
(\mathrm{GPa})\end{array}$ \\
\hline GR-FSH-1 & 25.29 & 2.51 & 240 & 4.86 \\
GR-FSH-2 & 24.85 & 2.41 & 280 & 5.00 \\
GR-FSH-3 & 24.94 & 2.45 & 280 & 4.76 \\
\hline
\end{tabular}

Table I.21 Specifications of Kevlar/epoxy specimens in 10" off-axis shear test.

\begin{tabular}{ccccc}
\hline $\begin{array}{c}\text { Specimen } \\
\text { ID }\end{array}$ & $\begin{array}{c}\text { Width } \\
(\mathrm{mm})\end{array}$ & $\begin{array}{c}\text { Thickness } \\
(\mathrm{mm})\end{array}$ & $\begin{array}{c}\text { Length } \\
(\mathrm{mm})\end{array}$ & $\begin{array}{c}(i \\
(\mathrm{GPa})\end{array}$ \\
\hline K-TSH-1 & 12.34 & 1.84 & 330 & 1.86 \\
K-TSH-2 & 13.55 & 1.84 & 330 & 1.72 \\
\hline
\end{tabular}




\section{Appendix J}

\section{LOAD-DEFLECTION CURVES OBTAINED FROM THE VSM}
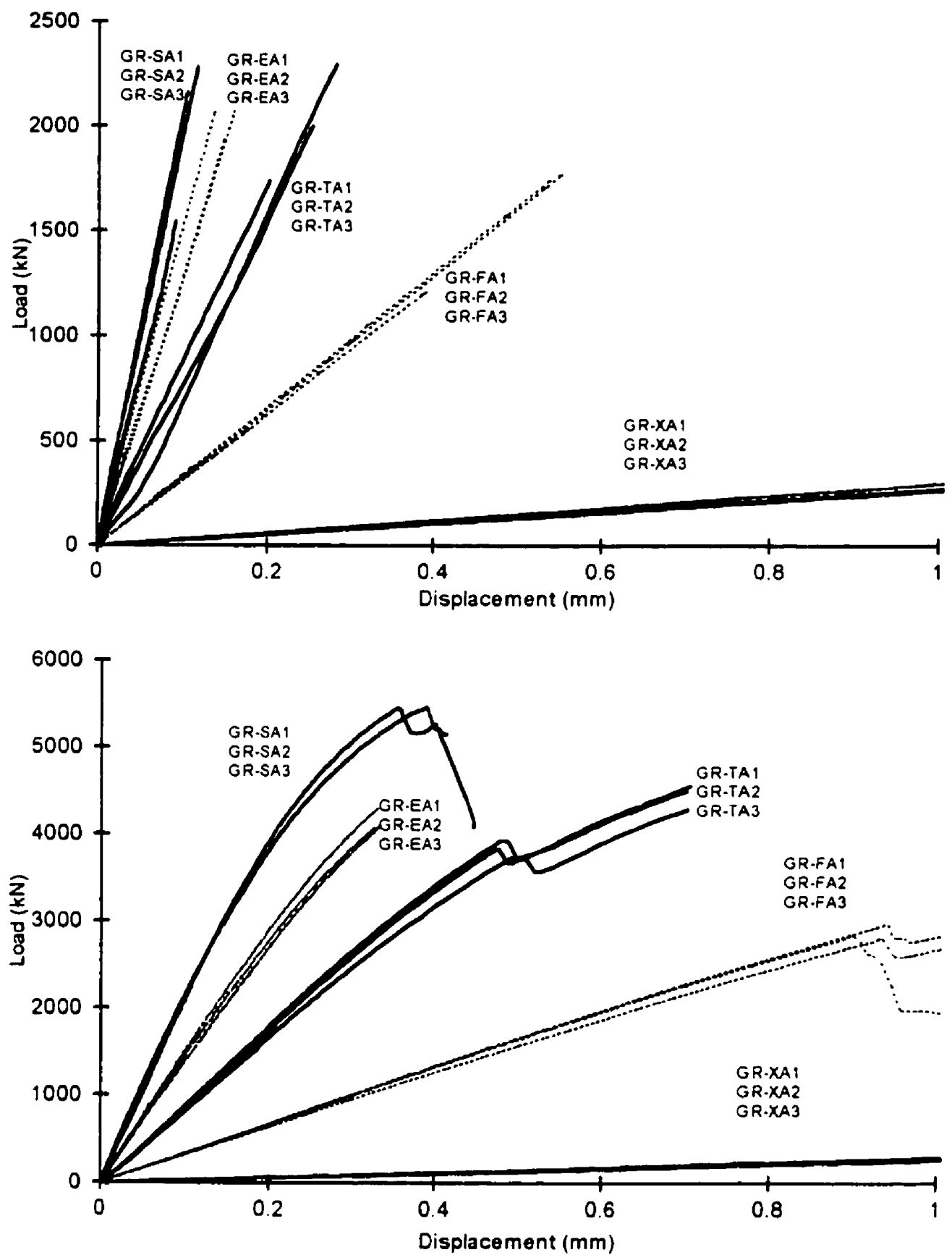

Figure J. I Load-deflection curves obtained for graphite/epoxy GR-1 specimens. Top: First loading. Bottom: Second loading. 

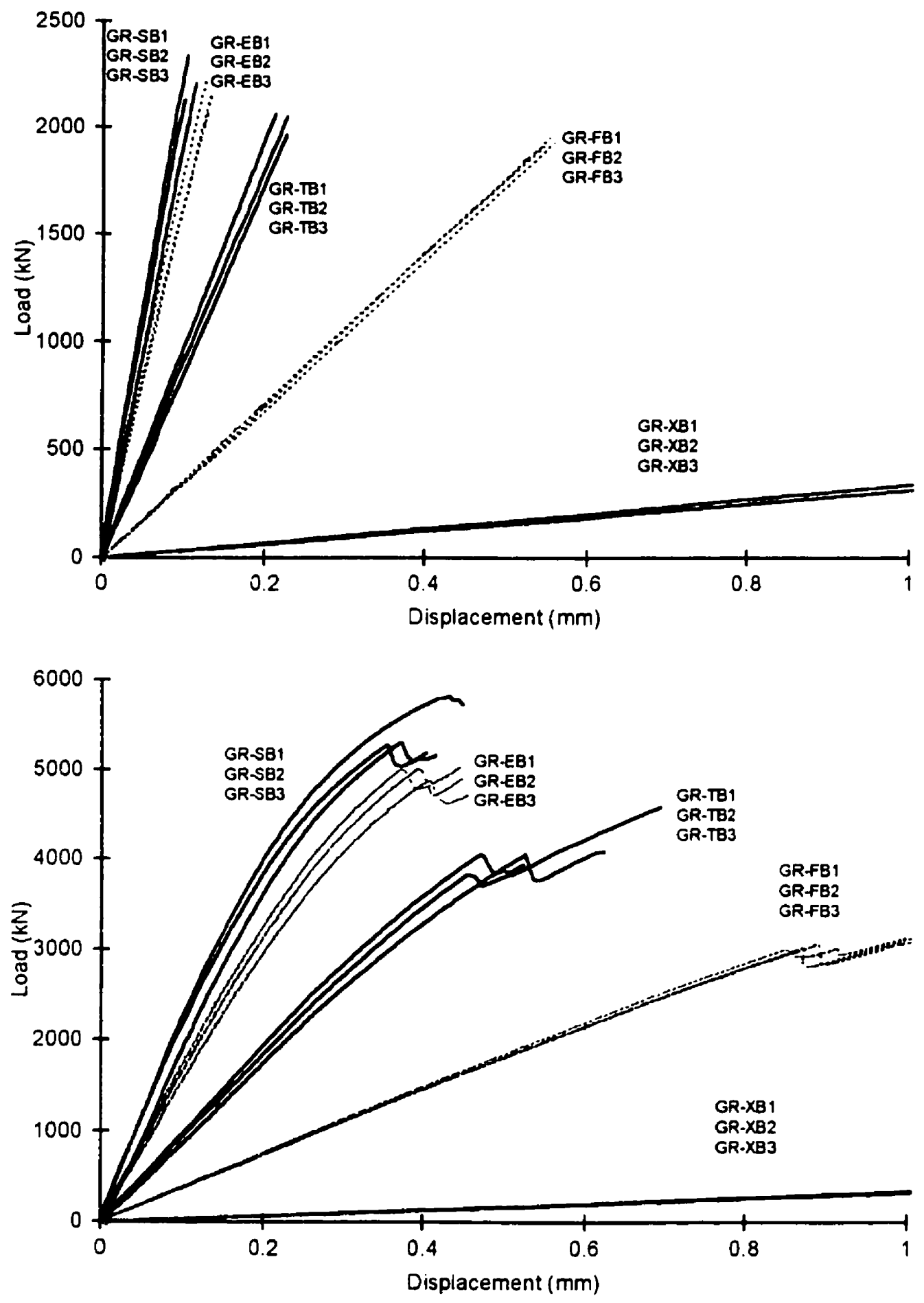

Figure J.2 Load-deflection curves obtained for graphite/epoxy GR-2 specimens. Top: First loading. Bottom: Second loading. 

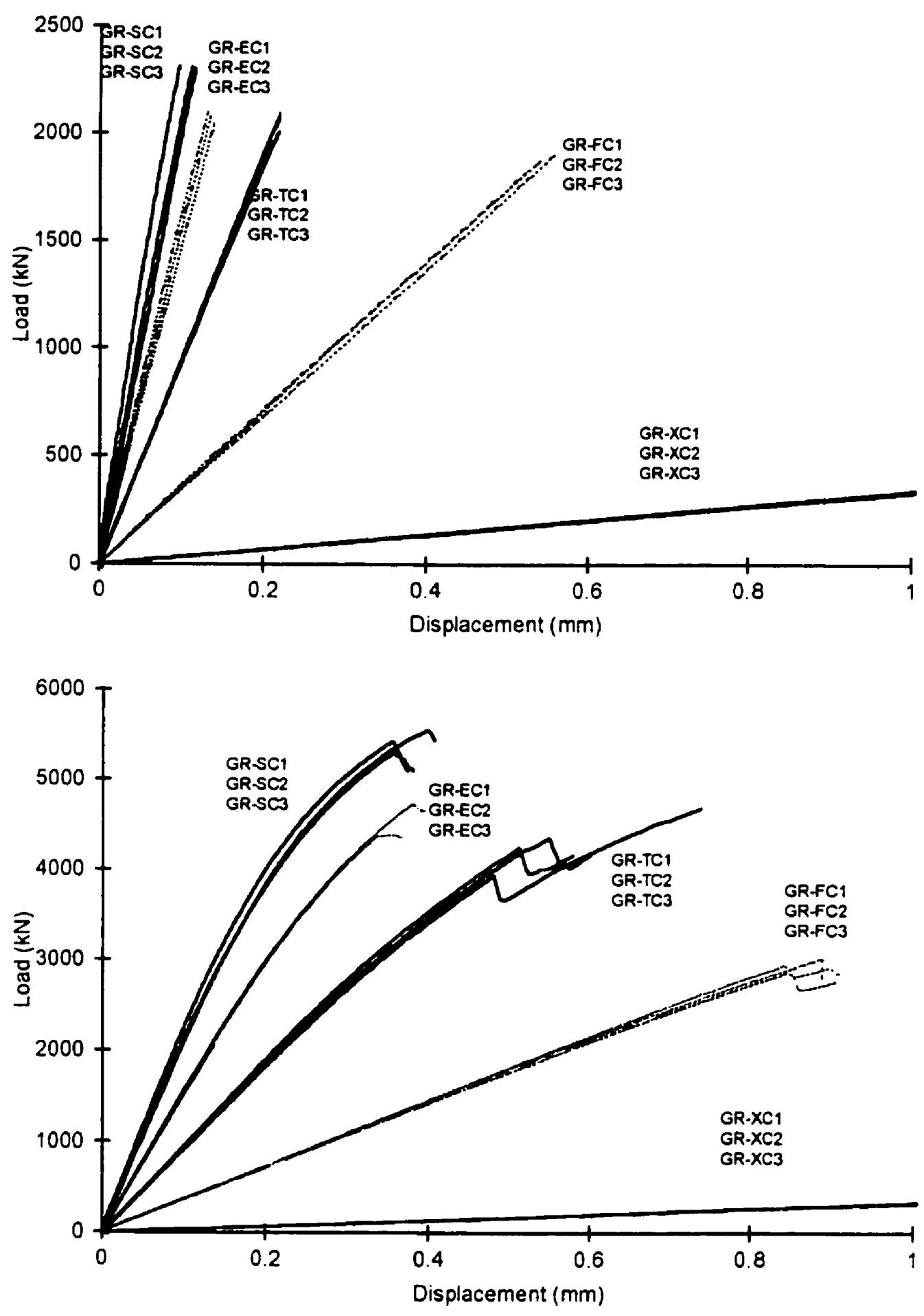

Figure J.3 Load-deflection curves obtained for graphite/epoxy GR-3 specimens. Top: First loading. Bottom: Second loading. 

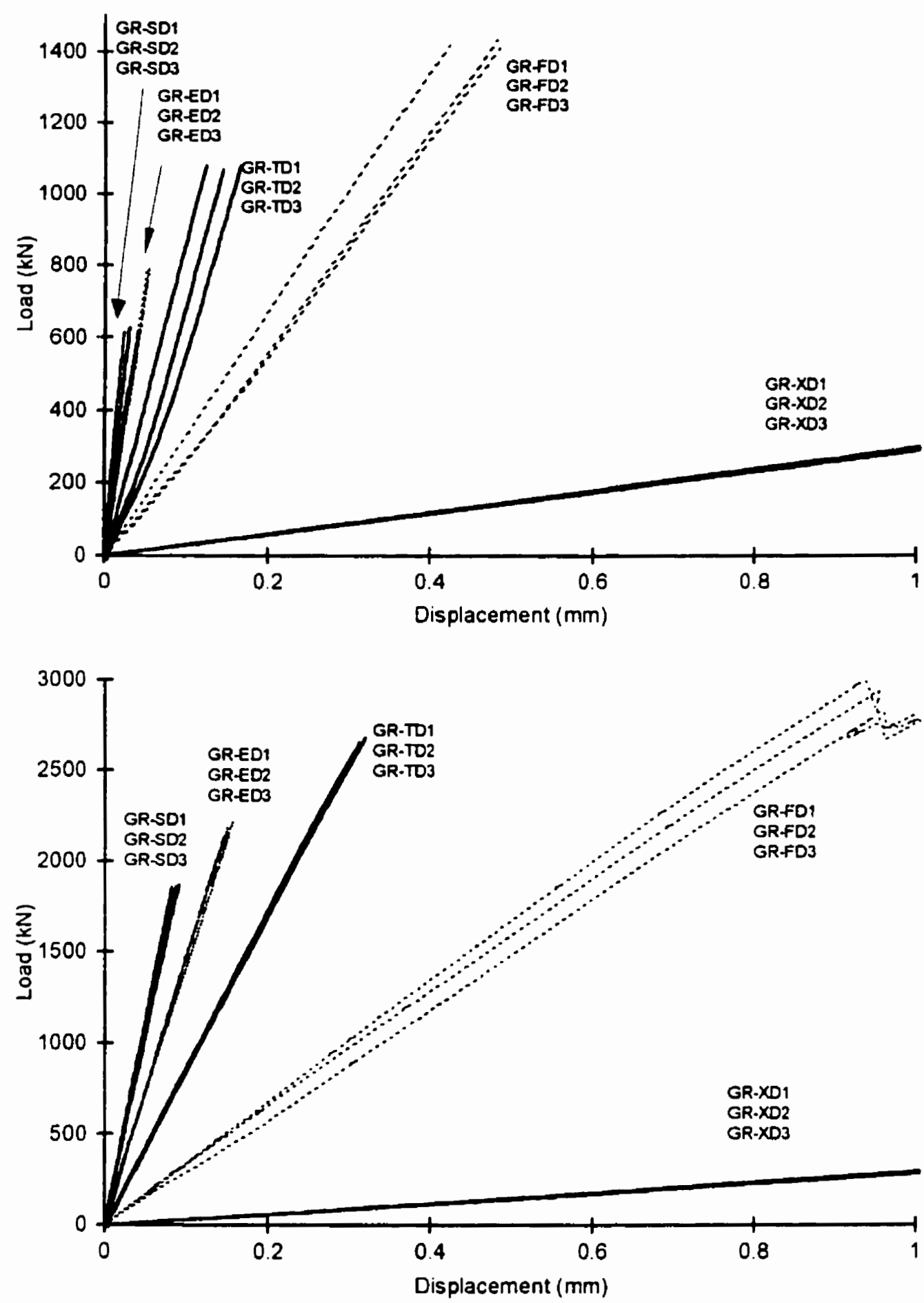

Figure J.4 Load-deflection curves obtained for graphite/epoxy GR-4 specimens. Top: First loading. Bottom: Second loading. 

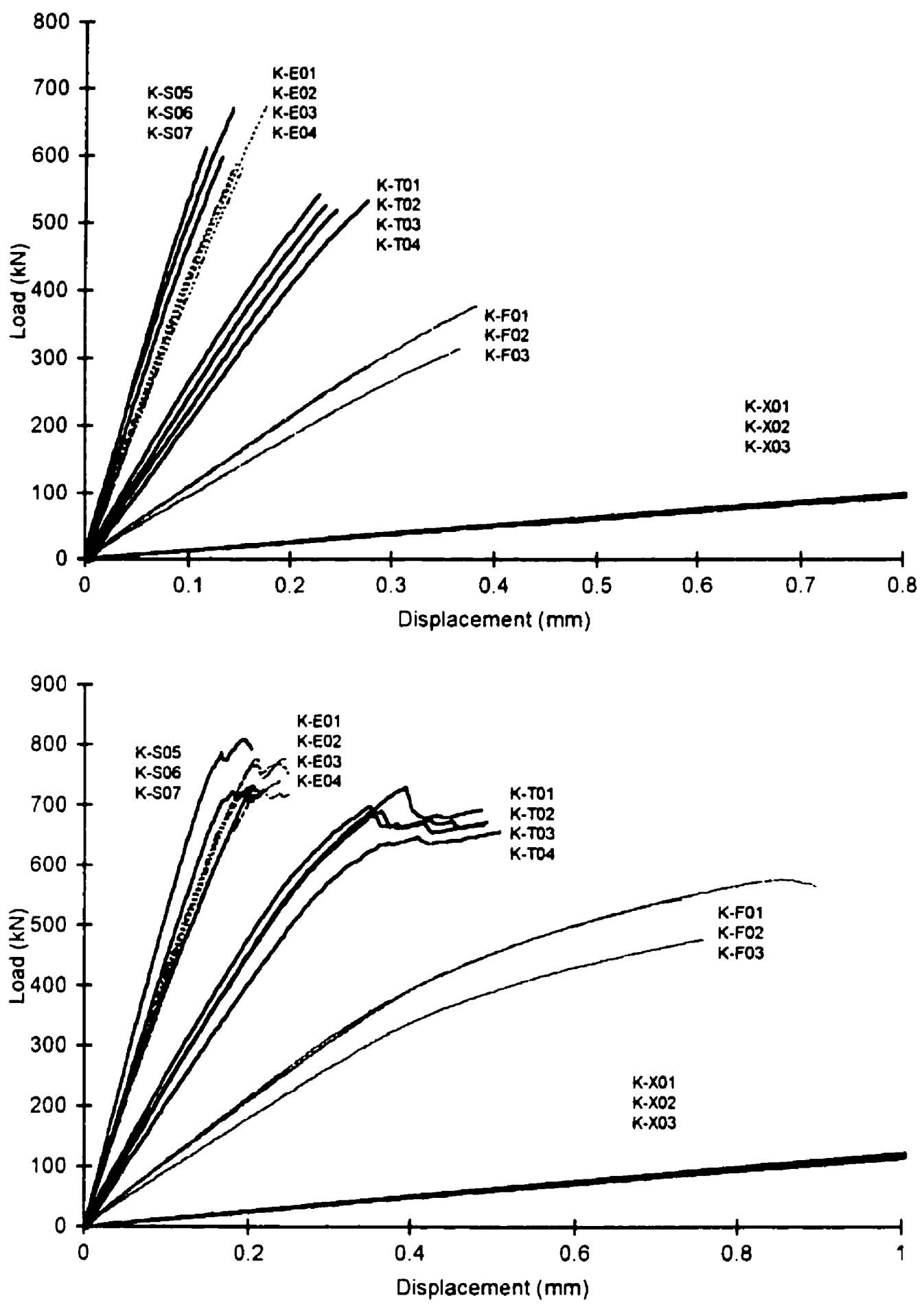

Figure J. 5 Load-deflection curves obtained for Kevlar/epoxy K-1 specimens. Top: First loading. Bottom: Second loading. 

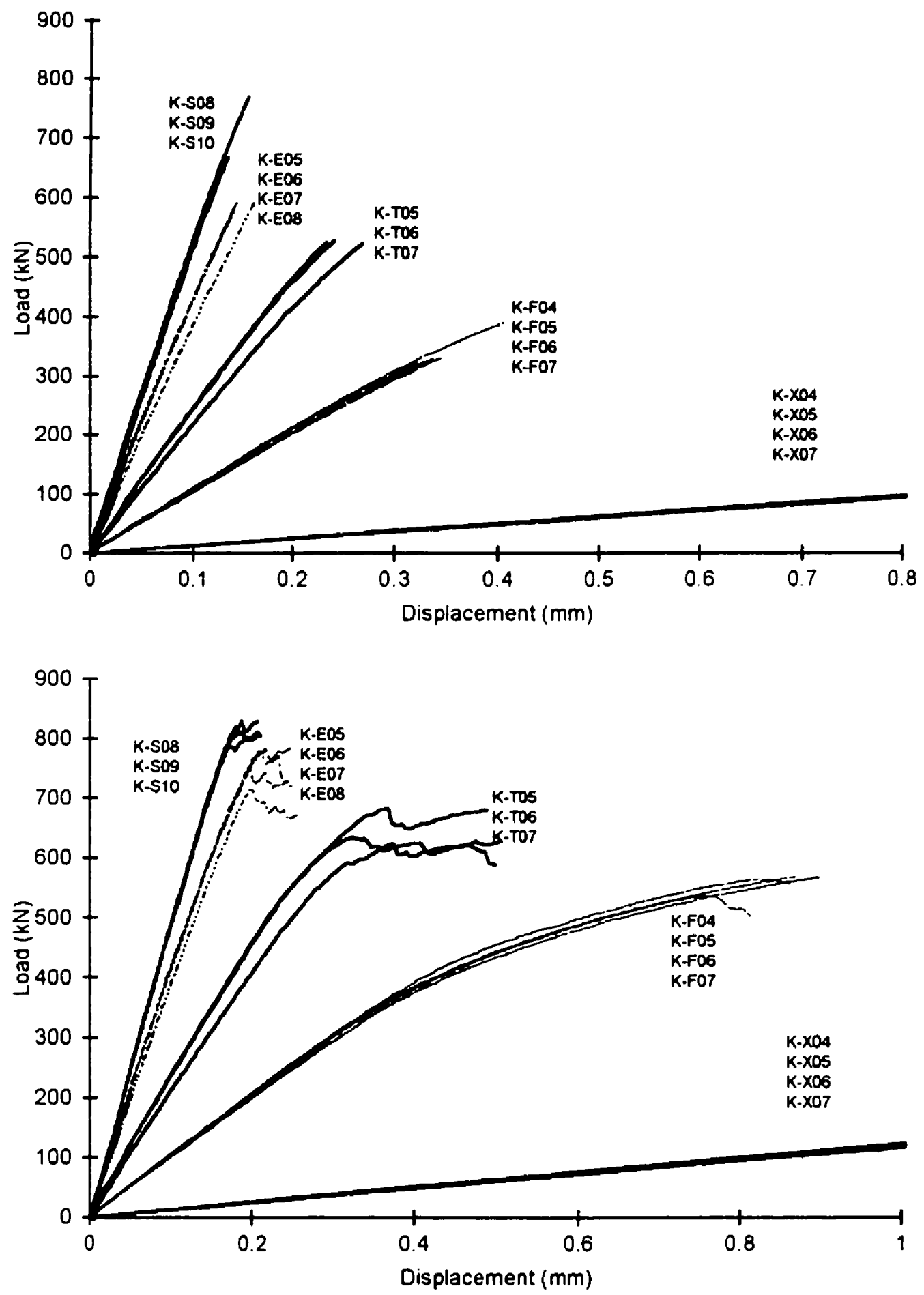

Figure J.6 Load-deflection curves obtained for Kevlar/epoxy K-2 specimens. Top: First loading. Bottom: Second loading. 

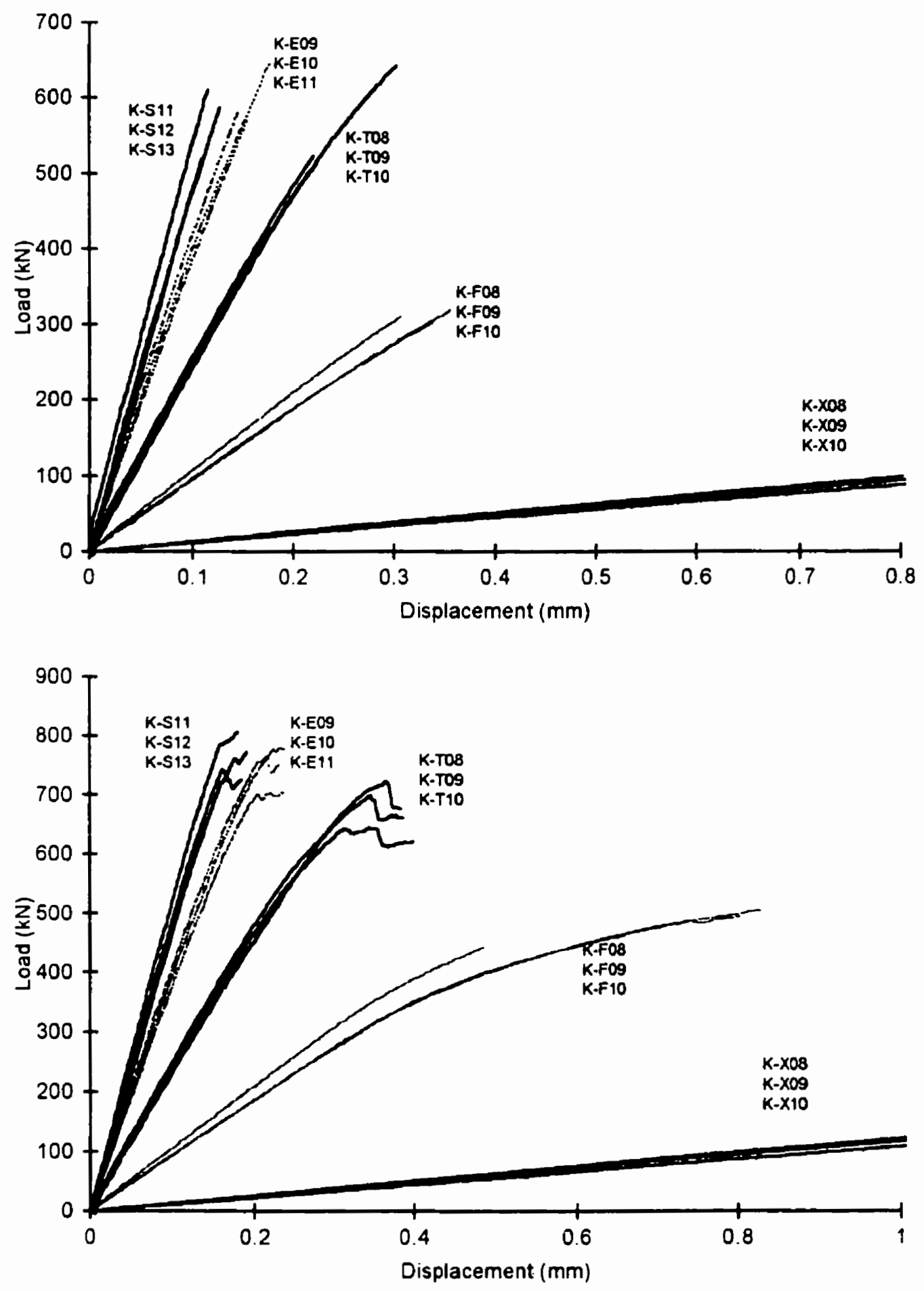

Figure J. 7 Load-deflection curves obtained for Kevlar/epoxy K-3 specimens. Top: First loading. Bottom: Second loading. 

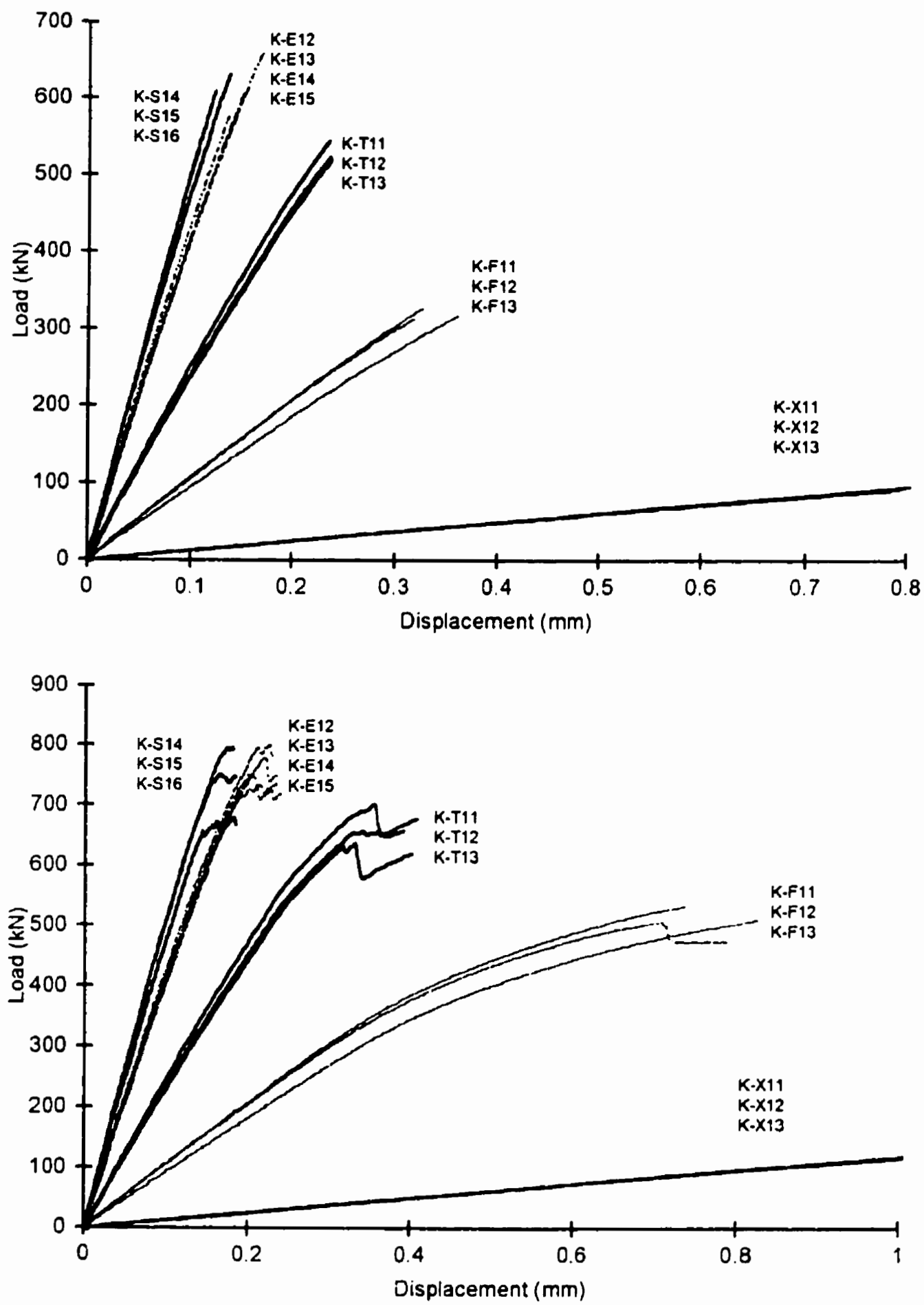

Figure J. 8 Load-deflection curves obtained for Kevlar/epoxy K-4 specimens. Top: First loading. Bottom: Second loading. 

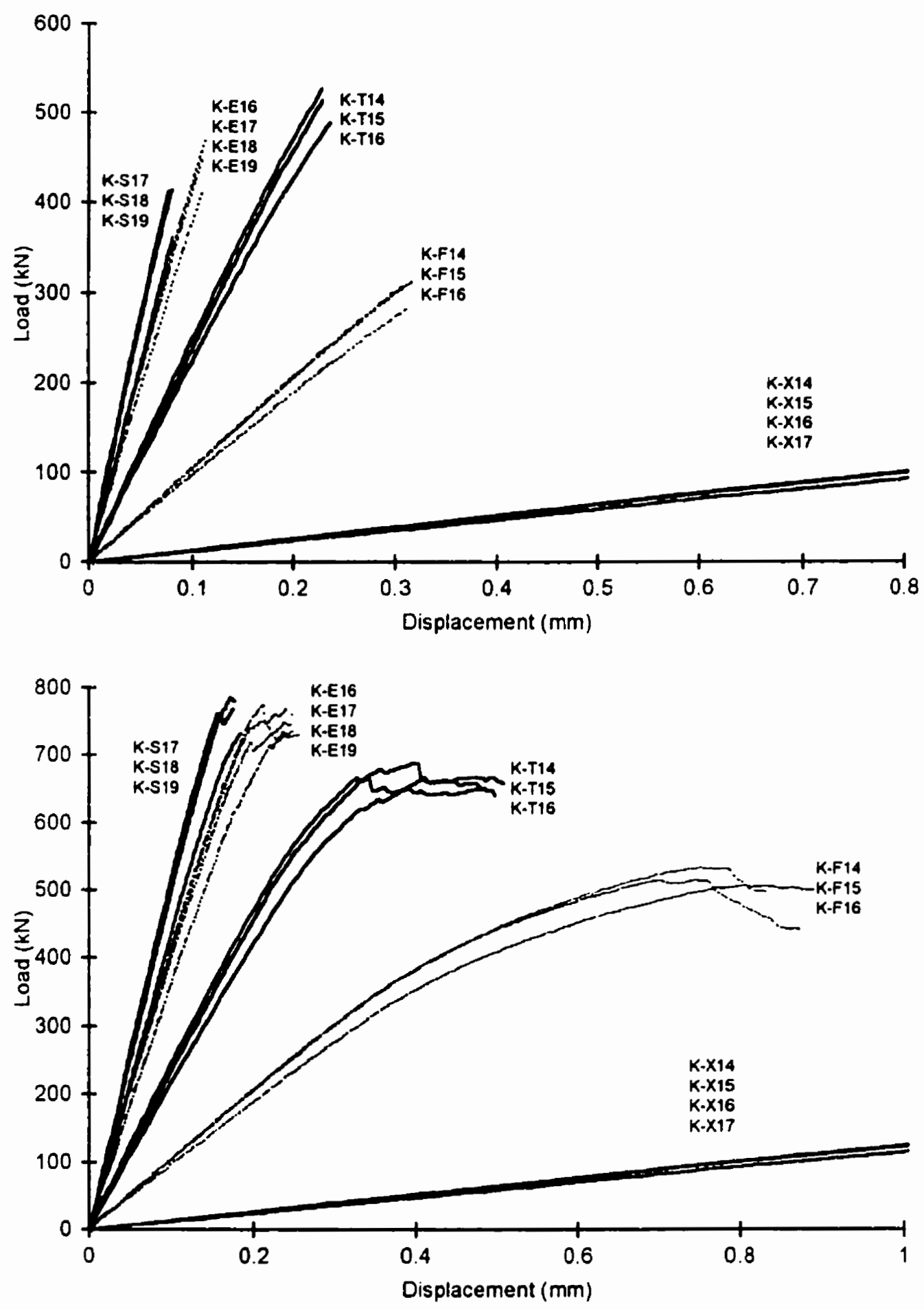

Figure J.9 Load-deflection curves obtained for Kevlar/epoxy K-5 specimens. Top: First loading. Bottom: Second loading. 

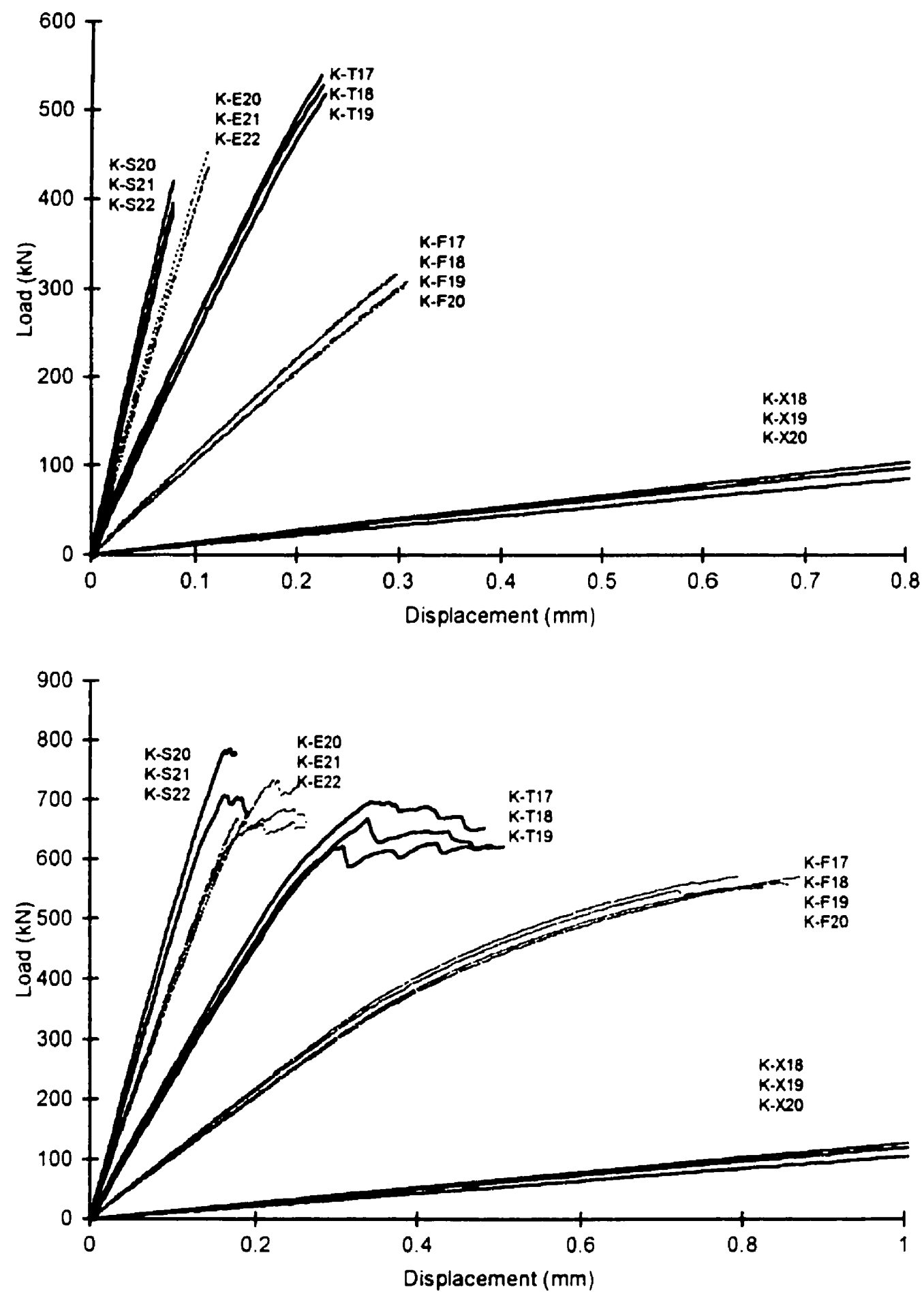

Figure J.10 Load-deflection curves obtained for Kevlar/epoxy K-6 specimens. Top: First loading. Bottom: Second loading. 

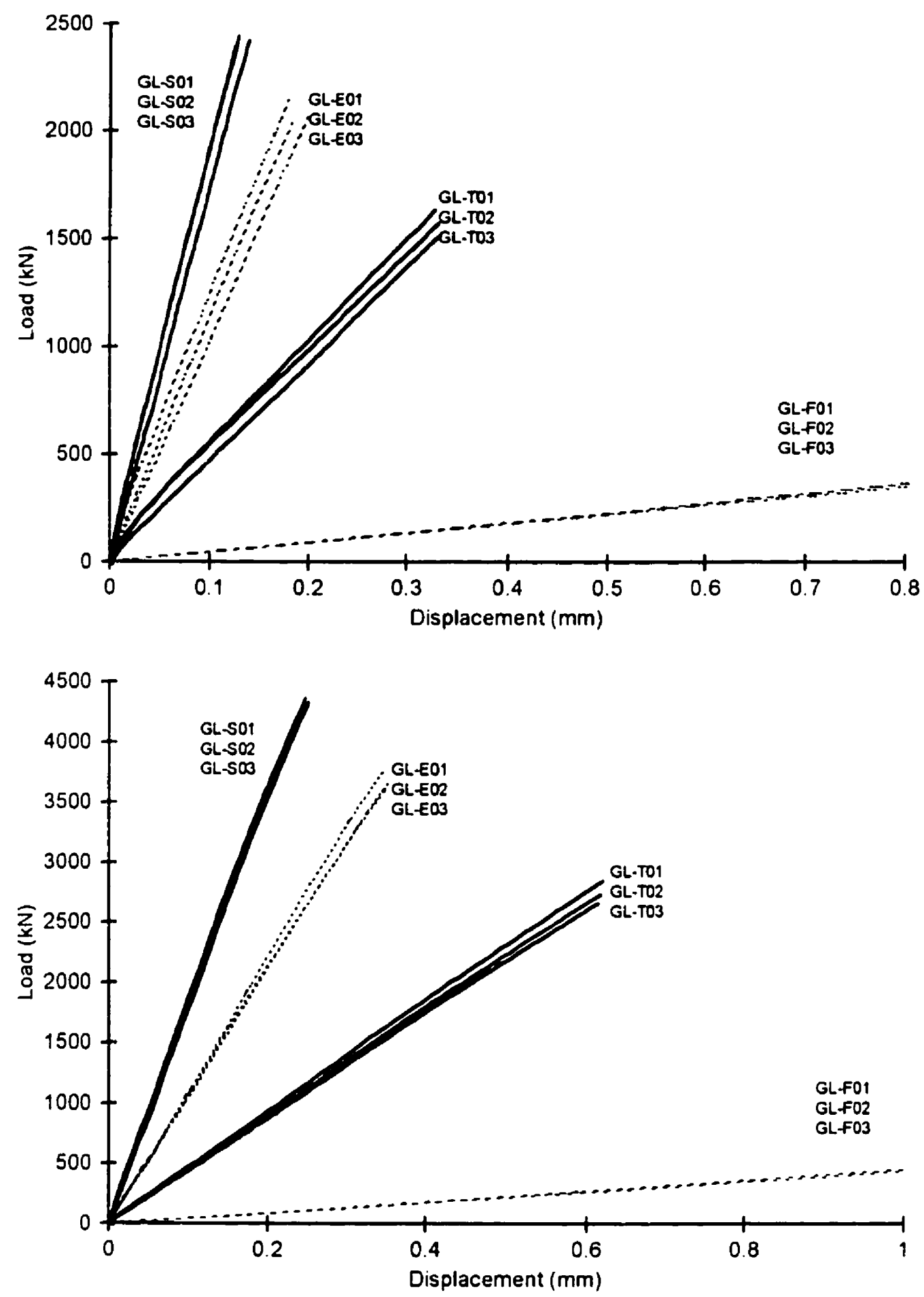

Figure J. 11 Load-deflection curves obtained for E-glass/epoxy GL-1 specimens. Top: First loading. Bottom: Second loading. 

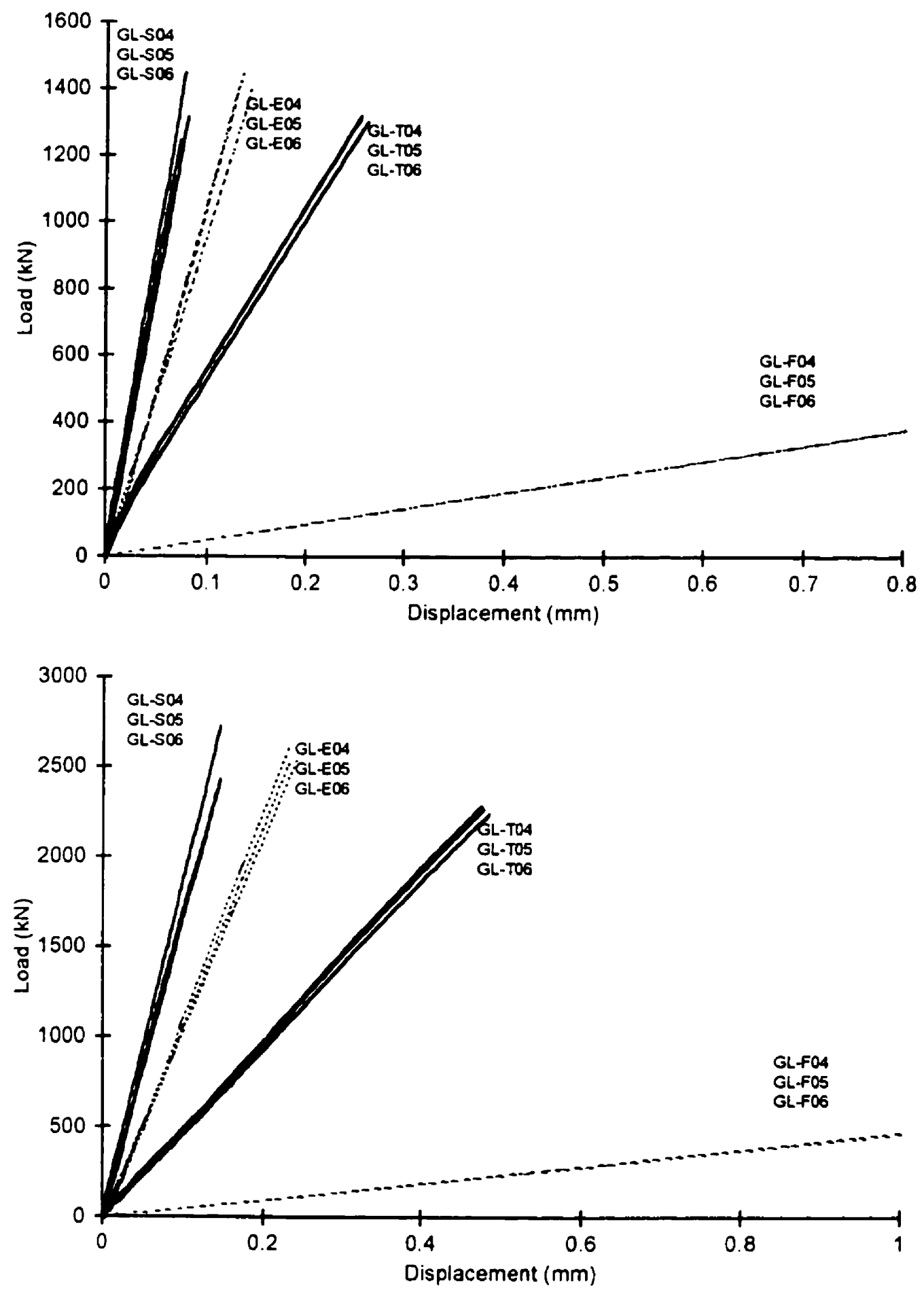

Figure J. 12 Load-deflection curves obtained for E-glass/epoxy GL-2 specimens. Top: First loading. Bottom: Second loading. 

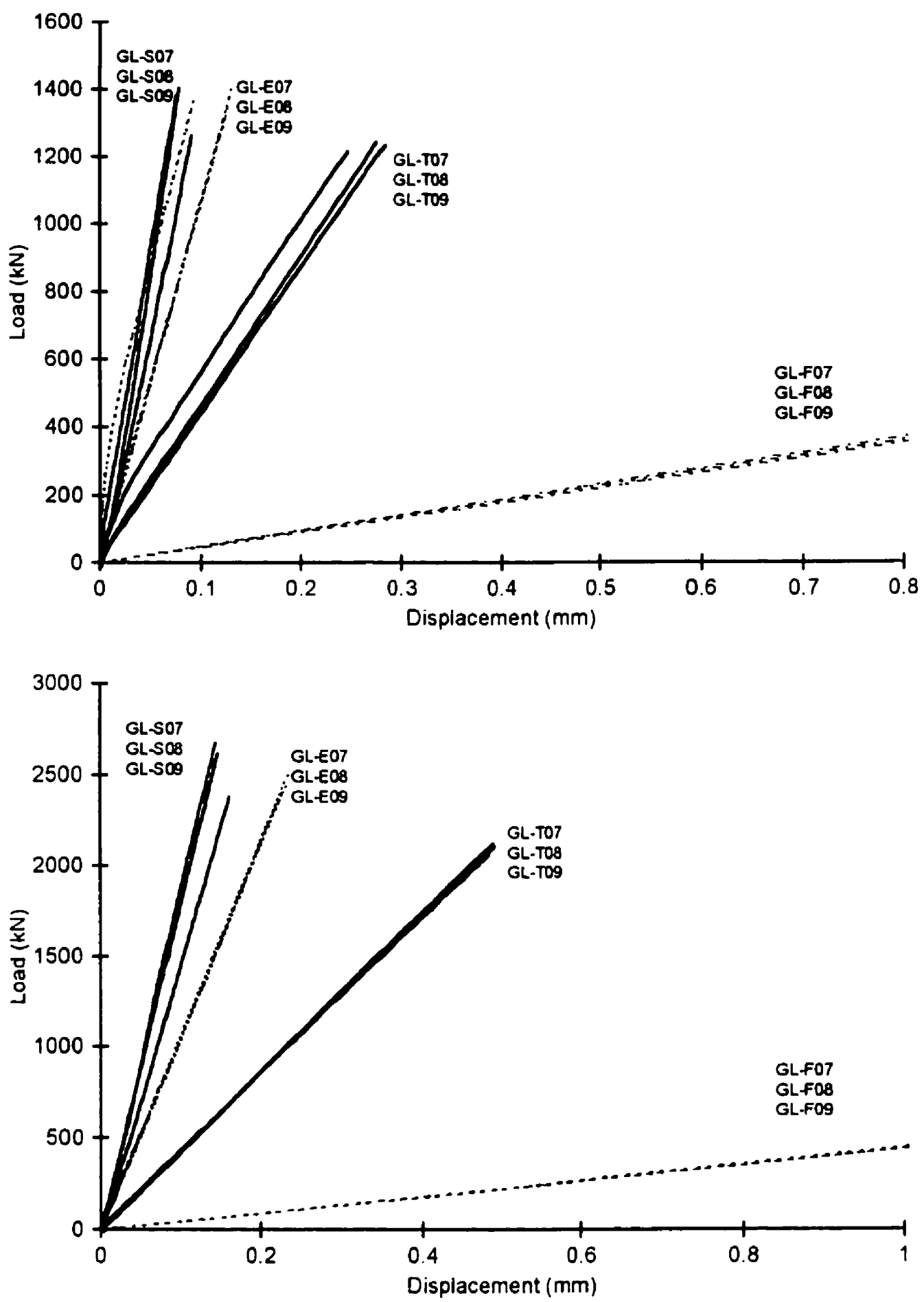

Figure J. 13 Load-deflection curves obtained for E-glass/epoxy GL-3 specimens. Top: First loading. Bottom: Second loading. 

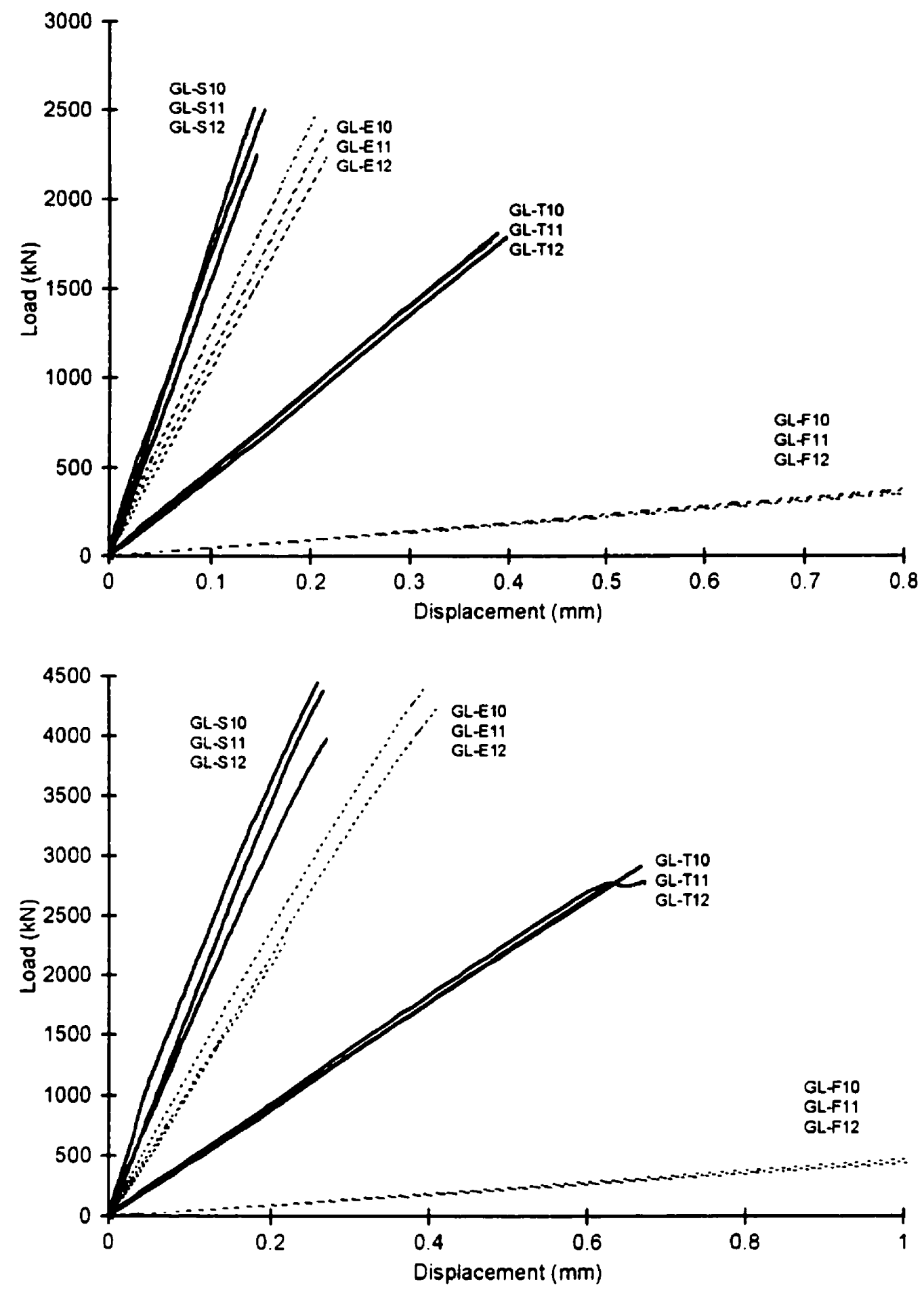

Figure J.14 Load-deflection curves obtained for E-glass/epoxy GL-4 specimens. Top: First loading. Bottom: Second loading. 

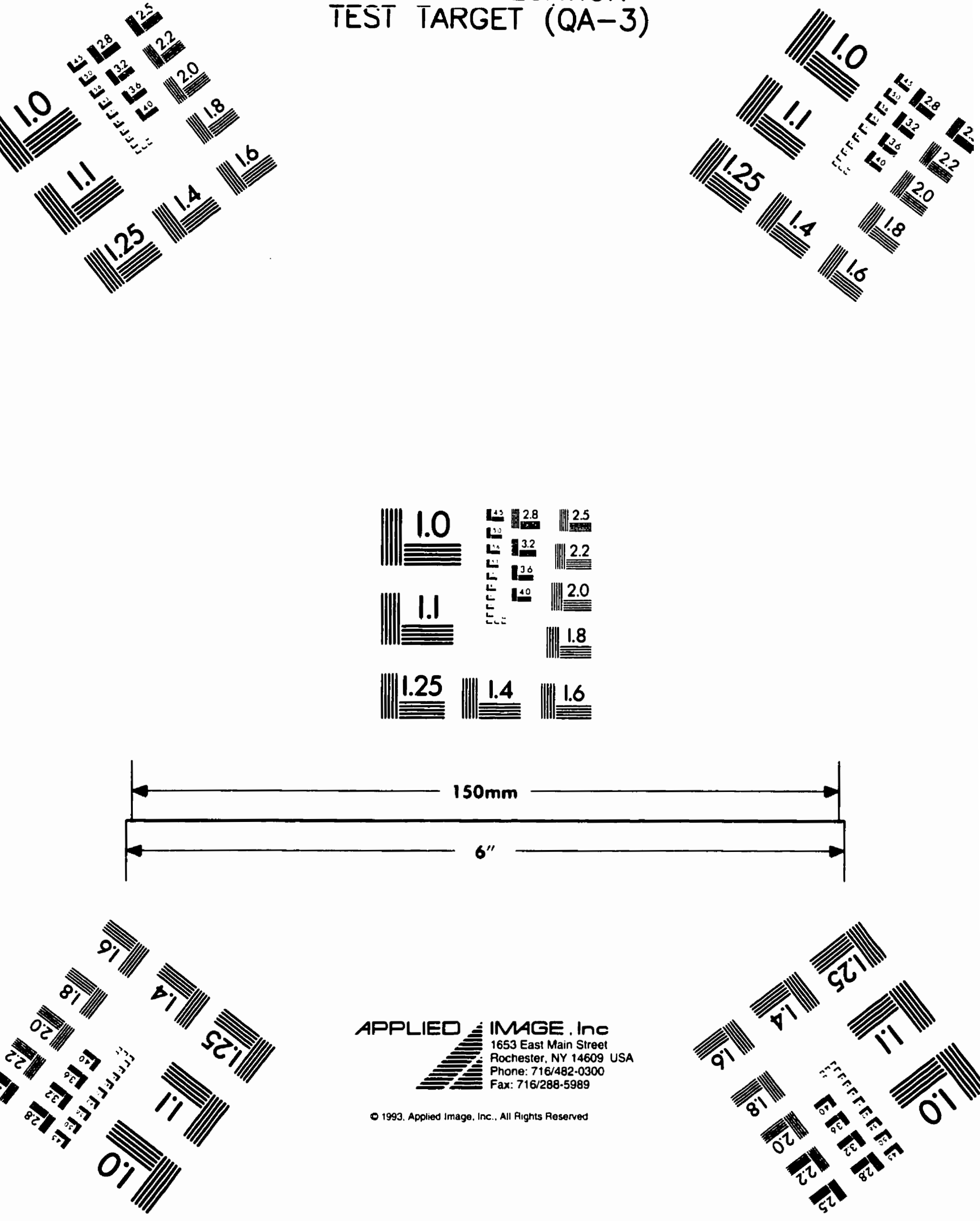